\title{
COMPARISON STUDY OF SEASAT SCATTEROMETER \\ AND CONVENTIONAL WIND FIELDS
}

\author{
by \\ Kristine Holderied \\ B.S., Oceanography \\ United States Naval Academy \\ 1984 \\ SUBMITTED IN PARTIAL FULFILLMENT OF THE \\ REQUIREMENTS FOR THE DEGREE OF \\ MASTER OF SCIENCE \\ at the \\ MASSACHUSETTS INSTITUTE OF TECHNOLOGY \\ and the \\ WOODS HOLE OCEANOGRAPHIC INSTITUTION
}

September, 1988

This thesis is not subject to U.S. copyright.

Signature of the author

Joint Program in Oceanography,

Massachusetts Institute of Technology -

Wooda Hole Oceanographic Institution

Tertified by

Carl Wunsch

Thesis supervisor

Accepted by

Carl Wunsch

Chairman, Joint Committee for Physical Oceanography.

Massachusetts Institute of Technology -

Woods Hole Oceanographic Institution. 


\title{
COMPARISON STUDY OF SEASAT SCATTEROMETER AND CONVENTIONAL WIND FIELDS
}

by

\author{
Kristine Holderied \\ Submitted to the Massachusetts Institute of Technology- \\ Woods Hole Oceanographic Institution Joint Program \\ in September, 1988 in partial fulfillment of the \\ requirements for the Degree of Master of Science
}

\begin{abstract}
A demonstrated need exists for better wind field information over the open ocean, especially as a forcing function for ocean circulation models. Microwave scatterometry, as a means of remotely sensing surface wind information, developed in response to this requirement for a surface wind field with global coverage and improved spatial and temporal resolution. This development led to the 1978 deployment of the SEASAT Satellite Scatterometer (SASS). Evaluations of the three months of SEASAT data have established the consistency of SASS winds with high quality surface wind data from field experiments over limited areas and time periods. The directional ambiguity of the original SASS vectors has been removed by Atlas et al. (1987) for the entire data set, and the resulting SASS winds provide a unique set of scatterometer wind information for a global comparison with winds from conventional sources.

A one-month (12 August to 9 September 1978) subset of these dealiased winds, in the western North Atlantic, is compared here with a conventional, pressure-derived wind field from the 6-hourly surface wind analyses of the Fleet Numerical Oceanographic Center (FNOC), Monterey, CA. Through an objective mapping procedure, the irregularly spaced SASS winds are regridded to a latitude-longitude grid, facilitating statistical comparisons with the regularly spaced FNOC wind vectors and wind stress curl calculations. The study includes qualitative comparisons to synoptic weather maps; calculations of field statistics and boxed mean differences; scatter plots of wind speed, direction, and standard deviation; statistical descriptions of the SASS-FNOC
\end{abstract} difference field, and wind stress curl calculations.

The SASS and FNOC fields are consistent with each other in a broad statistical sense, with wide scatter of individual values about a pattern of general agreement. The FNOC wind variances are slightly smaller than the SASS values, reflecting smoothing on larger spatial scales than the SASS winds, and the SASS mean values tend to be slightly higher than the FNOC means, though the increase is frequently lost in the large scatter. Exceptions to the pattern of relatively small consistent variations between the two fields are the pronounced differences associated with extremely strong winds, especially during Hurricane Ella, which traveled up the East Coast of the United States during the latter part of the study period. These large differences are attributed mainly to differences in the inferred positions of the pressure centers and in the response at the highest wind speeds $(>20 \mathrm{~m} / \mathrm{s})$. The large statistical differences between the SASS and FNOC fields, present under high wind conditions, may yield significantly different ocean forcing, especially when the strong winds persist over longer periods of time. Under less intense wind conditions, usually prevailing over the ocean, the two fields correspond well statistically and the ocean responses forced by each should be similar.

Thesis supervisor: Dr. Carl Wunsch

Cecil and Ida Green Professor of Physical Oceanography

Secretary of the Navy Research Professor 
ßs

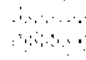


Table of contents

Abstract ..................... . . . . . . . .

Chapter 1: Introduction. . . . . . . . . . . . . . . . 4

Chapter 2: Background. . . . . . . . . . . . . . . . 10

Wind velocity and wind stress. . . . . . . . . . . . . 12

Ocean wave spectrum and radar backscatter. . . . . . . . 16

Empirical U-to- $\sigma^{\circ}$ relation. . . . . . . . . . . . . 18

Chapter 3: SEASAT Scatterometer (SASS). . . . . . . . . . . 21

SASS geophysical algorithm. . . . . . . . . . . . . 23

SASS performance evaluations. . . . . . . . . . . . . 26

SASS data reprocessing. . . . . . . . . . . 28

Chapter 4: Data description and handling. . . . . . . . . 30

Objective mapping procedure . . . . . . . . . . . . . . 32

Chapter 5: Results and discussion. . . . . . . . . . . . . . . 39

Field statistics. . . . . . . . . . . . . . . . .42

Ungridded Atlas winds. . . . . . . . . . . . . . . .42

Mapped Atlas winds. . . . . . . . . . . . . .43

FNOC winds. . . . . . . . . . . . . . 45

Boxed mean differences. . . . . . . . . . . . . . . 47

Scatter plots of wind speed and direction. . . . . . . . . . 49

Difference field statistics . . . . . . . . . . . . 53

Wind stress curl. . . . . . . . . . . . . . 57

Chapter 6: Summary and conclusions. . . . . . . . . . . 60

Appendix 1. Aspects of atmospheric boundary layer theory. . . . . . .65

Appendix 2. Ocean waves and energy transfer. . . . . . . . . 71

Appendix 3. Aspects of radar scatter theory. . . . . . . . . . 73

References . . . . . . . . . . . . . . . . . . . . .

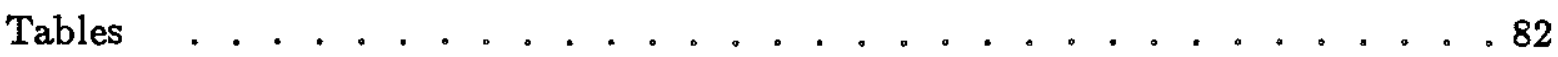

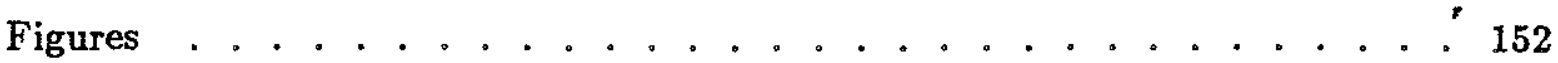

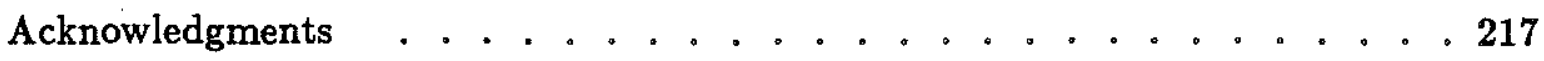




\section{Introduction}

The large-scale oceanic circulation has been extensively studied, especially over the past 100 years, and considerable advances have been made in theoretical understanding of the processes involved. A critical part of this study is the attempt to understand the forcing that drives the circulation. The two major driving forces are the stress due to wind blowing over the ocean surface and the buoyancy-driven motions due to thermohaline processes. The directly wind-driven circulation is confined to, and dominates, flow in the upper layers of the ocean. It is primarily horizontal motion, but convergence and divergence of this flow give rise to vertical motion, creating regions of upwelling or downwelling. Theoretical study of the wind-driven circulation was motivated initially by attempts to account for observed surface currents, starting with the work of Nansen (1898), Ekman (1905), and Sverdrup (1947). Further progress was associated with attempts to explain westward intensification, reflected in strong western boundary currents (Stommel, 1948; Munk, 1950). This work established our basic understanding of how wind stress drives the ocean circulation, and subsequent developments are reviewed by Veronis (1981) and Pond and Pickard (1983).

While wind-forcing is accepted as the primary driving force in the upper layers, themohaline forces also play a significant role in ocean circulation, especially in the lower layers where they tend to dominate. In this case, water movements are associated with changes in density, either from temperature or salinity changes. In the ocean these changes normally occur as an increase in density at the surface, either directly through cooling or indirectly through freezing and subsequent production of more saline water. Such processes cause a buoyancy-driven vertical flow that is followed by horizontal flow due to continuity requirements or to geostrophic currents resulting from the changes in density. Thermohaline processes are frequently associated with changes in weather and climate, and may also be strongly influenced by the wind field, especially in terms of surface heat fluxes and downward mixing in the surface layer. Therefore, the wind field plays a most critical role, both directly and indirectly, 
in forcing the ocean circulation, and its specification is crucial to reaching a more complete understanding of that circulation through theory and model developments.

However, the development and verification of these theories and their application to global circulation models are hampered by the current lack of good wind fields over the open ocean. The most frequently used fields for wind-forcing are averaged estimates of the wind stress, available both globally (Hellerman, 1967,1968; Han and Lee, 1983; Hellerman and Rosenstein, 1983) and for particular regions (for example: Bunker, 1976; Hastenrath and Lamb, 1977; O'Brien and Goldenberg, 1982). These climatological values are averaged across monthly, seasonal, and annual periods, generally from all available ship reports in the chosen period, extending over a considerable length of time and employing some wind speed to stress conversion. In the case of the Hellerman and Rosenstein (1983) analyses, for example, ship reports from 1870 to 1976 were used to form monthly averages, and stress was computed from wind speed based on a wind speed and atmospheric stability dependent drag coefficient. Many uncertainties still exist in the details of the wind speed to wind stress relationship (discussed in Section 2), and these parameterization issues limit the accuracy of the stress fields. However, a greater limitation is posed by the long averaging periods, which result in a considerable loss of information on the temporal variability of the wind field. In addition to the wind stress fields derived from longterm ship reports, daily maps of wind speed derived from surface pressure analyses, supplemented by ship and buoy reports, are available. While these are available much more frequently than the averaged stress fields, they are not direct measurements of the surface wind field, but inferred from the much smoother quantity of pressure. Spatial resolution is considerably reduced due to these inherently large pressure field scales, and small, intense circulations may be lost in the broader scale unless they are observed independently.

The insufficient amount of wind measurements, sparse global coverage, and restricted scales of conventional wind information available limit the studies that can be done. In particular, conventional sources generally provide winds only at larger scales (> $1000 \mathrm{~km})$, geostrophically from surface pressure fields, and at very small 
scales $(<10 \mathrm{~km})$, from ship and buoy reports. There is virtually no resolution of the intermediate spatial scales, which contain significant energy and are important to atmospheric forcing of the ocean (Freilich and Chelton, 1986). The conventional data also lack sufficient temporal resolution for modeling the temporal variability of the upper ocean, especially over larger areas. The absence of information at shorter time and space scales forces reliance on long time-averaged data for modeling purposes. These averages are of some use in studying the mean circulation, but lose much of the variability needed to drive the more complex models. Some studies of the temporal and spatial variability in realistic wind fields, as well as of the impact of that variation on ocean motions, have been conducted, though the studies are necessarily limited by the coarse resolution of climatological wind fields (for example: Willebrand, 1978; Willebrand et al., 1980; Muller and Frankignoul, 1981). The forcing effect of wind stress on the ocean surface is a major determinant for ocean waves and upper ocean currents. Knowledge of these motions is crucial to many fields, including basic oceanographic research, climate studies, and practical ocean operations. The interrelation of these motions and the wind forcing can be more fully evaluated only if an accurate, global wind field is available for input into the numerical models. Conventional sources of wind field information, including many different types of observations and analyses, do not currently provide sufficient information for this purpose, and a repeated, global measurement of wind stress (or velocity) over the ocean would therefore be a unique contribution (O'Brien, 1982).

The microwave scatterometer, yielding wind velocity as inferred from surface roughness measured by reflected radiation, was developed to provide this missing information. Mounted on aircraft or, more effectively, on satellites, this instrument has the potential to provide a more accurate, variable, global wind field at higher temporal and spatial resolutions than currently available. The SEASAT Satellite Scatterometer (SASS), launched in 1978, was the first operational scatterometer system to be flown. The three months of data returned from that mission is a unique set of information that has yet to be repeated, since no scatterometer has flown since. Details of basic scatterometer theory, the SEASAT mission, and SASS itself are given in Section 2. 
The wind forcing information derived from scatterometer measurements provides both new research opportunities with the expanded information it makes available, as well as very useful information for immediate use in practical operations. Improved specification of the wind forcing would allow ocean modelers to better estimate ocean behavior, and therefore reach a more thorough understanding of upper ocean physics. The scatterometer can resolve winds on the intermediate spatial scales and the global, shorter time scales that are missing in conventional data. This information would contribute greatly to modeling of the temporal and spatial variability of the upper ocean (Freilich and Chelton, 1986). The ocean is forced by the wind stress, both directly, and indirectly through mass field adjustments, and the stress field must be incorporated into any realistic ocean model. Current parameterizations of thermodynamic forcing, including the surface fluxes of sensible and latent heat, use aerodynamic bulk formula models that incorporate wind velocity. So, better wind field information would improve our understanding and modeling capability of both wind stress and thermodynamic forcing. In the absence of good forcing fields from conventional sources, oceanographers are restricted to artificial forcing fields, at best adjusting the sparse information currently available to obtain reasonable results from the model. Some of the new ocean modeling opportunities opened up by the more accurate, extensive wind fields provided by the scatterometer include: 1) improved ocean basin models, 2) improved wind forcing related process studies, 3) improved prediction and monitoring of ocean climate phenomena such as El Niño, and 4) better input for practical, daily ocean forecasting models.

Scatterometer derived wind fields will also have valuable applications to meteorological research and weather prediction, and to a better understanding of processes at the air-sea interface. Assimilation of scatterometer data may have a significant impact on numerical weather prediction, through both model initialization and subsequent updates (Cane et al, 1981; Yu and McPherson, 1979; 1981; Duffy and Atlas, 1986; Harlan and O'Brien, 1986). The additional information provided by the scatterometer is particularly important in data sparse areas, such as the Southern Ocean. Scatterometer derived wind fields also have important applications to naval 
and commercial ocean operations. These include tactical weather forecasting, acoustic conditions forecasting, surf forecasting for amphibious operations, ship routing, and warnings of potentially catastophic situations. More commercial uses include oil platform design, drilling schedules, and commercial fishing schedules. The potential cost savings associated with these scatterometer wind applications are summarized in O'Brien (1982).

Better vector wind stress fields will be needed to drive the increasingly sophisticated ocean models, designed to resolve temporal and spatial variability on finer scales. The choice of a particular wind field for this forcing is crucial when we want to more accurately establish the influence of the "real" surface winds on ocean circulation. At that point it becomes crucial to pick the "best" wind field available, in terms of accurately reproducing the actual winds with sufficient resolution at the desired scales. Even if it is not possible to determine which is best in an absolute sense, it is important to at least reach a good understanding of the differences between the various wind fields available, especially in their ultimate impact on model output. In particular, when conventional and scatterometer derived fields are considered as alternative forcing functions, the presence or absence of significant differences between the two can influence the decision to deploy a scatterometer in the first place. If no significant differences are found between the two fields, over the time and space scales of interest, then the effort and expense necessary to develop and maintain an operational scatterometer is not justified, since it would not significantly augment information already available. This study does not intend to address the more comprehensive question of the need for a scatterometer, assuming, as discussed briefly above, that this requirement is already well-established (Brown, 1983;1986; O'Brien, 1982). Rather, assuming that the scatterometer wind fields will be available and will therefore compete with conventional winds as model input, this work seeks to examine the differences between these fields in a more detailed fashion. This is accomplished by qualitatively and quantitatively comparing two such fields, the conventional surface wind analyses from the Fleet Numerical Oceanographic Center (FNOC) in Monterey CA, and SEASAT Scatterometer winds from R. Atlas at the 
Goddard Space Flight Center (GSFC) (Atlas et al., 1987), obtained through the Jet Propulsion Laboratory (JPL), Pasadena, CA. An overview of satellite scatterometry is provided in Section 2 to introduce some basic atmospheric boundary layer and microwave backscatter concepts. Section 3 describes the SEASAT mission and SASS, and Section 4 provides a general description of the various data used and outlines the objective mapping procedure used to regrid the scatterometer winds. Section 5 provides more detailed wind field statistics and various quantitative comparisons between the scatterometer and conventional winds, including boxed mean differences, scatter plots of various quantities, difference field statistics, and wind stress curl calculations. Section 6 contains the summary and conclusions. 


\section{Background}

The development of microwave scatterometer theory is based on studies of the atmospheric boundary layer, radar scatter, and ocean surface motions. Some of the basic concepts from these areas that apply directly to scatterometer work are reviewed here. The complex nature of the air-sea boundary makes quantifying and measuring physical processes there a difficult problem. Wind stress, $\tau$, represents the transfer of momentum from the atmosphere to the ocean. This transfer of momentum slows the air near the ocean surface, creating an atmospheric boundary layer. The vertical profile of wind velocity in the atmosphere is dominated by molecular processes in the few millimeters closest to the sea surface, with turbulent motions, governed by friction and buoyancy, dominating the rest of the surface layer (Stewart, 1985). At higher levels the surface effect diminishes and the geostrophic general circulation of the atmosphere governs the flow. The region most critical to scatterometer work is the 20 meters closest to the surface, and concentration on this region affects assumptions made about the relative dominance of various processes. In general, the wind velocity profiles are derived analytically, combining a logarithmic layer with an Ekman spiral modified by secondary flow for winds greater than $5 \mathrm{~m} / \mathrm{s}$ (Brown, 1986). The various parameters and details behind this theory are discussed in more detail below and in Appendix 1.

The study of atmospheric and surface boundary layers is based on formulas and empirical correlations that were originally derived from empirical studies. Many field experiments have been conducted to investigate the relevant processes, and then to adjust the formulas and refine the empirical constants (see Stewart, 1985 for partial review). Three quantities, the fluxes across the sea surface of horizontal momentum, sensible heat, and latent heat, are of particular interest in the $1 \mathrm{~cm}$ to $10 \mathrm{~m}$ surface layer where the surface effect dominates. Within this turbulent atmospheric surface layer, the fluxes are approximately constant with height and the wind and temperature profiles are logarithmic - with adjustments for stability (Halbertstam, 1980). Much study has been devoted to deriving the fluxes from wind speed and temperature 
profiles. The similarity concepts of Obukhov (1946) and initial experiments of Monin and Obukhov (1954) are incorporated in most of the profile formulas. The vertical profile of wind velocity, and its relation to surface drag, or stress, strongly influences the transfer of energy from atmosphere to ocean. Wind stress, $\tau$, is accepted as the major driving force for large-scale ocean circulation. However, practical considerations have dictated that, rather than being measured directly, the wind stress is normally derived from wind velocity, $\mathrm{U}$, usually measured at some distance above the ocean surface. This is a highly complicated relationship, dependent on the complex dynamics of the boundary layer at the air-ocean interface.

The scatterometer uses a measure of the surface roughness, determined from the reflection of microwave radiation, as an inferred measure of the air-sea energy transfer. The surface roughness can be connected to a particular geophysical parameter, such as wind velocity or wind stress, as well as to the backscattered power that forms the scatterometer signal. In practice, a direct relation is sought between the backscatter and a geophysical parameter, commonly chosen to be the wind velocity at some height above the surface. However, this relation has no direct physical basis, and is determined empirically. The theoretical justification for the empirical relation comes rather from our understanding of various parts of the process, and the interconnection of quantities such as the wind velocity, wind stress, ocean wave spectrum, and backscattered radiation. The review of these basic concepts below is divided into three broad areas, the U-to- $\tau$ relation, the impact of wind stress on the ocean wave spectrum, and the relation between the ocean wave spectrum and reflected radiation. These are examined in turn, followed by a discussion of their practical, but indirect application in the direct relation between wind velocity and reflected radiation. In a sense this discussion can be seen as describing two paths to the same goal, the first a multi-step, theoretical process and the second a single, empirical step, where the latter path is dependent on the principles established by the former. 
Wind velocity and wind stress

The U-to- $\tau$ relation is determined by converting the wind at some reference height, $U_{\text {ref }}$, through the boundary layer, to a surface stress, $\tau$. This connection between the wind velocity profile and wind stress are of primary importance to scatterometry. The interfacial momentum flux, or wind stress, can be defined as the average turbulent transfer of horizontal momentum from atmosphere to ocean by vertical air movements (O'Brien, 1982). For a constant wind, the wind stress is calculated from correlations between perturbations of the vertical and horizontal wind components, expressed as :

$$
\tau=\rho \overline{u w}
$$

where:

$$
\begin{aligned}
& \tau=\text { wind stress }\left(\mathrm{N} / \mathrm{m}^{2}\right) \\
& \rho=\text { air density }\left(\mathrm{kg} / \mathrm{m}^{3}\right) \\
& \mathrm{u}, \mathrm{w}=\text { horizontal and vertical velocity }(\mathrm{m} / \mathrm{s})
\end{aligned}
$$

(Stewart, 1985)

The averaging period must be long enough to form stable averages of the turbulent properties, yet short enough for mean conditions to be steady. Times of 40 minutes to 1 hour are usually chosen. Compounding the complex theoretical issues in this relation are the practical difficulties inherent in measuring the relevant quantities. The ocean surface is a difficult environment to work in, and the critical turbulent time and space scales are relatively short. To circumvent the constraints of direct measurement, a series of empirical relationships (bulk or aerodynamic formulae) have been developed. These relate the wind stress to the wind velocity at some height, with the usual form:

$$
\tau=\rho \mathrm{C}_{\mathrm{D}} \mathrm{U}_{\mathrm{ref}}^{2}
$$

where:

$$
\begin{aligned}
& C_{D}=\text { drag coefficient } \\
& U_{\text {ref }}=\text { wind velocity at some reference height }(\mathrm{m} / \mathrm{s})
\end{aligned}
$$


The exact formulation of the drag coefficient is still subject to considerable debate, and varies with wind speed and atmospheric stability, as discussed in Appendix 1. A commonly accepted formulation is that of Large and Pond (1981), which combines a constant value for $C_{D}$ at lower wind speeds $(<11 \mathrm{~m} / \mathrm{s})$ with an expression that is linearly dependent on wind speed at higher speeds (see Appendix 1 for the exact form).

The wind velocity profile near the surface varies sharply with height and depends on both $\tau$ and atmospheric stability. The transfer of heat and momentum depend on turbulence generated by both wind shear and buoyancy (affected by temperature and humidity stratification). Shear dominates near the surface and buoyancy becomes relatively more important at some height above the surface. The influence of stability is illustrated by considering the case of cold air blowing over warmer water. Air is heated from below and rises, causing instability in the air column and enhancing the turbulence due to just the mechanical shear of the wind stress. In the opposite case, when warm air blows over cold water, there is a suppression of turbulence as cooling from below stably stratifies the air column. The impact of wind stress and stability on motions in the turbulent layer is described quantitatively by MoninObukhov similarity theory, discussed in more detail in Appendix 1. A primary result from these theories is that, assuming that the influence of atmospheric stability is weak, the wind velocity profile is logarithmic with height for neutral stability, and simply shifted by stability corrections. Under unstable conditions, or for a strongly stratified atmosphere, these formulations are no longer valid, but the condition of weak stratification is generally satisfied over widespread areas, except in the tropics where strong convection cells are common.

Though considerable debate still exists on some details of the theory and on the exact formulation of the relation, there is a generally accepted understanding of the wind stress-to-velocity connection. The choice of wind velocity in particular, and if so, at what height, as the relevant parameter to relate to microwave backscatter is a still greatly debated issue. The neutral stability wind at $19.5 \mathrm{~m}$ was chosen for the original processing of SEASAT scatterometer data, based on anemometer heights 
aboard some weather ships. From boundary layer considerations, a better choice would be the wind velocity closer to the surface (such as at $10 \mathrm{~m}$ ), or the friction velocity. Another alternative parameter, using the wind just above the surface, is discussed in a later section. Though the "best" parameter has yet to be decisively chosen, for the initial evaluations made for SASS development, the most practical choice was wind velocity. The boundary layer wind velocity theories described here provide justification for that particular choice, as they connect the wind velocity to wind stress. A factor requiring further evaluation is the effect of stratification, especially since it can have a significant influence at all scales. The parameterizations discussed here hold for relatively weak stratification effects, but are questionable under conditions where atmospheric stratification plays an important role, such as in the tropics. To isolate these effects for future uses, widespread measurements are needed of both the currently available sea surface temperature measurements, and the not yet generally available air temperature measurements over the ocean (Atlas et al., 1986). A more detailed parameterization of the effects of stability, along with better sea surface and air temperature observations would significantly improve and extend the range of scatterometer wind algorithms.

Wind stress and the ocean wave spectrum

Given some stress at the ocean surface, the next step is to determine how this transfer of momentum from the air is reflected in the ocean wave spectrum. Understanding the processes behind this energy transfer is a fundamental problem of remote sensing. Important factors in the wind-wave relation include wave generation, wave-wave and wave-current interactions, wind strength, atmospheric stratification, sea surface temperature, and sea state. The ultimate goals are to determine how much energy is transferred from air to ocean, on what scales the transfer takes place, and how the energy is partitioned to various ocean features, in particular, waves and currents. Wind generated waves range from millimeters to hundreds of meters and, while there is considerable theoretical understanding of the longer gravity waves, the 
high-frequency end of the spectrum is much less well understood. Current theory holds that the short wavelengths reflect the surface response to the local wind field. As discussed in detail in the following section, scatterometers are designed to look at these wavelengths, in particular at the centimeter-length capillary waves. Some physical factors that influence the surface ripple field are: 1) the tangential and normal stresses at the surface, 2) the fraction of momentum transfer going into capillary waves, 3) the magnitude and direction of the swell, 4) ocean currents and mixing, 5) wave history, 6) hydrostatic stability (Davidson et al., 1981). The lack of definitive descriptions of open ocean capillary waves and their relation to the surface fluxes has hindered theoretical understanding in this area. Evidence also exists that only a portion of the air-sea momentum transfer goes into these waves, and the assumption that the short waves reflect the entire momentum transfer, and therefore the wind stress, is not supported by any complete quantitative theory (Brown, 1986). Partial explanations are available for various processes, especially wave generation, growth, and interaction, but many critical effects have yet to be modeled, and a single, unified theory does not exist. Short waves are associated with skin friction-induced smallscale stress, and therefore do not account for the direct momentum transfer through form drag on long waves from pressure fluctuations (Brown, 1986). The partitioning of momentum transfer between shear stress and form drag is discussed in more detail in Appendix 2. Other unaccounted for factors that may distort the local wave field to local wind relationship include swell, usually generated at more distant locations and unrelated to local winds, and momentum transfer to ocean currents, thus unavailable for wave generation. At present, the influence of uncorrelated swell and the momentum loss to currents are assumed to be small and are neglected. A short review of some theories and experiments on the basic physics behind the transfer of energy from air to water is given in Appendix 2. The concepts and studies discussed here provide the still rough theoretical understanding of the wind-wave relation used to justify scatterometer development. These present interpretations are supported by the generally good results gained from scatterometer measurements made thus far. Further refinements of the assumed stress-to-wave spectrum relation are still needed 
to resolve effects such as the time response of wave formation, interference from other waves, and the effect of sea slicks.

Ocean wave spectrum and radar backscatter

The final step is to determine the ocean wave spectrum from information on surface roughness gained from reflected radiation, or backscatter. The interaction of electromagnetic radiation with the surface provides information on surface features, depending on the wavelengths of the radiation and the features. Extracting surface information from the power of the backscatter signal therefore requires knowledge of both scattering theory and surface characteristics. Details of relevant surface scattering principles are provided in Appendix 3. The primary radiation measure is the normalized radar cross section (NRCS), $\sigma^{\circ}$, a ratio of the reflected to incident energy across a unit surface area. The crucial problem is to characterize the shape of $\sigma^{\circ}$ for a given sea surface parameter, generally chosen to be the ocean wave spectrum. Scatter from the wavy ocean surface is described by two physical mechanisms, specular and Bragg (or resonant) scatter, which depend on the incidence angle (angle between the radar beam and local surface vertical) and wavelength of the incident radiation, and on the wavelengths of the surface waves. Appendix 3 contains further descriptions of these scatter mechanisms, including the incidence angle dependence and ranges, and the adjustments needed to accomodate the multiple scales of oceanic motion. The most useful return for scatterometry is the resonant scatter from capillary waves, yielding both speed and direction information on the wave spectrum, and by association, on the surface stress or wind velocity.

These basic scattering concepts are complicated by the complex conditions actually present on the sea surface. Wave tank and field experiments have shown spikes in backscatter returns due to sharp-crested waves that are close to breaking (Kwoh and Lake, 1984). The effect on $\sigma^{\circ}$ of rain striking the surface is significant, but not well understood. The capillary waves tend to be damped by rainfall, thus decreasing backscatter return, but rain-generated ripples also appear, increasing the 
radar return. Some experimental evidence has shown $\sigma^{o}$ to increase with rainfall (Moore et al., 1979), but more study is needed to determine the magnitude of this effect. Sea slicks can significantly affect backscatter as well (Huhnerfuss et al., 1983), and recent work further demonstrates the impact of wave slope and atmospheric stratification on the radar return (Keller et al., 1985). At higher wind speeds, when the surface is confused, with significant foam and spray coverage, the application of Bragg scatter theory is questionable and new models may be necessary (Atlas et al., 1986).

While microwave backscatter theory is reasonably well understood in terms of electromagnetic radiation principles, the precise connection to the short wave spectra is not well specified. Rather, as discussed in detail in Section 3, the assumed relation between the two is used to justify empirical models that relate backscatter to wind velocity and various parameters of the radar signal. Though these models produce realistic results and have been used with much success, they shed little light on the physical processes that link microwave backscatter and ocean wave spectra. Also, as discussed by Plant (1986), the current formulations of short wave spectra derived from physical principles (eg: Phillips, 1977; Kitaigorodskii, 1983; and Phillips, 1985) are not directly applicable to scatterometry, since they cannot account for the dramatic increase in the backscatter signal with the increase in wind speed (and therefore surface roughness). Addressing this question, Plant (1986) derives a form for the short wave spectrum that incorporates long wave-short wave interactions as well as wind input and dissipation. The expression for the short wave spectra is valid to second order in long wave slope, and is then used, for the case of locally generated seas, to develop an expression for $\sigma^{\circ}$, also valid to second order in long wave slope. This formulation allows a more detailed investigation of the effects of long wave slope and propagation on backscatter, through the tilting and hydrodynamic modulation of the short waves, but it is still a considerable simplification of actual ocean conditions. Assuming that the relevant parameter is the backscatter from capillary waves, in the real ocean these ripples (and therefore the associated $\sigma^{\circ}$ signal) are modulated by ocean currents,internal waves, and sea state, and subject to tilt effects from longer 
waves. Surface currents cause variations in $\sigma^{o}$ through their effects on waves, including changing the direction of propagation and amplifying wave amplitudes by local velocity convergences. The effect of internal waves is not that pronounced at this scale, but sea state can have a significant impact (Atlas et al., 1986). The influence of sea state is especially important in rougher conditions, when foam, spray, and breaking waves disrupt the surface and contaminate the backscatter return, often as signal spikes.

In the absence of detailed knowledge of the actual long wave field, the optimum conditions for scatterometry occur when equilibrium is reached by those waves approximately one order of magnitude longer than the capillary waves (Atlas et al., 1986). These conditions are assumed in scatterometer algorithm development, and though this steady state condition is typical for the open ocean, it will not hold near significant circulation features, such as fronts. In order to completely specify the modulation and tilt effects for future algorithms, more extensive and more highly resolved in situ ocean wave spectra information is needed. In addition to refining the empirical scatterometer relations, this information could also provide a means of developing and validating theories on the physical processes discussed here. These scattering concepts, though simplifications of realistic conditions, are supported by the few experimental studies available (see Stewart, 1985 for summary). The difficulty of making simultaneous measurements of ocean wave spectra and backscatter has necessarily limited the amount of experimental evidence. While many questions still remain to be answered, there is increasing physical understanding of microwave backscatter, of the short ocean wave spectrum, and of the connection between them. Future study will be directed to quantifying the effects on microwave backscatter of rain, wave slope, sea surface temperature, currents, internal waves, and breaking waves.

\section{Empirical U-to- $\sigma^{\circ}$ relation}

The theories described above are used to establish a physical foundation, through a convoluted, multiple-relation process, on which to connect microwave backscatter 
surface wind velocity. The response of short capillary waves to environmental changes at the air-sea interface, as measured by radar backscatter, is used to determine wind speed and direction. Given the theoretical support provided by these concepts, the practical application is to use that basis to justify seeking a direct relation between wind and the measured radar cross-section. The direct relation is established in three steps: 1) isolate the critical parameters, 2) establish the basic form of the equation (ie: power law, drag law, etc.), and 3) describe the detailed aspects of the model through empirical coefficients. Empirical studies have shown the dependence of $\sigma^{\circ}$ on wind speed and wind direction relative to the horizontal antenna pointing angle as well as on the incidence angle, polarization, and frequency of the radar signal (Plant, 1986): Wind speed dependence is described by an adjusted power law while directional dependence is characterized by an approximately $\cos (2 \chi)$ dependence on the relative azimuth angle, $\chi$. The empirical coefficients are derived by tuning the basic equation to a set of data, for which both the known and unknown variables have been found. An obvious limitation to this process is that tuning to a particular data set constrains the backscatter-to-wind relation to the range of conditions present in that data. The specific details of the $\sigma^{\circ}$-to-wind relation used for SEASAT scatterometer data are described in Section 3. It should be mentioned that the radar cross-section has been shown to correlate well with both wind velocity and friction velocity (Liu and Large, 1981). However, as mentioned above, due to the need for conventional measurements to validate the empirical relation, and to the relative unavailability of wind stress measurements, a wind velocity dependence was chosen for SEASAT. One problem with current formulations is an inconsistency between the parameterizations for neutral wind and friction velocity. The current choices of power law relations to backscatter for both are not compatible with the expected connection between the two for a neutrally stratified atmosphere (Pierson et al., 1986).

Recent theoretical and experimental evidence indicates that the capillary wave spectrum, and by assumption the microwave backscatter, correlates better with a 
near-surface wind field characteristic, $R$, than with either the neutral wind or friction velocity (Pierson et al., 1986). This new parameter is expressed as:

$$
R=\left(\frac{U\left(\frac{\lambda}{2}\right)}{C(\lambda)}-1\right)^{2}
$$

where:

$\mathrm{U}\left(\frac{\lambda}{2}\right)=$ average wind at half a Bragg wavelength above the

surface

$$
\begin{aligned}
& C(\lambda)=\text { phase speed of Bragg waves } \\
& \lambda=\text { Bragg wavelength }
\end{aligned}
$$

(Pierson et al.,1986)

This term is related to the normal stresses, and correlation with short wave spectra depends on the assumption that normal stresses dominate tangential stresses in wave generation (Atlas et al., 1986). Unlike a direct correlation of backscatter to friction velocity, use of $R$ requires definition of the wind profile and specification of a drag coefficient, or friction velocity and roughness length (Pierson, et al., 1986). So the application of $\mathrm{R}$ with scatterometer data is similar to using the neutral stability winds, but with a characteristic that is more physically related to the surface roughness. These concepts may improve future scatterometer missions, but SEASAT data was processed with a $\sigma^{\circ}$-to- $U$ relation and that will be emphasized here. Despite many unresolved issues, the available SASS results have demonstrated that useful correlations between microwave backscatter and a wind or surface roughness parameter can be found (Brown, 1986). 


\section{SEASAT Scatterometer (SASS)}

A considerable microwave backscatter history culminated in the development of the SEASAT scatterometer, commencing with experiments on radar sea clutter during and after World War II. The initial proposal to use microwave backscatter to determine winds at the ocean surface came from Moore and Pierson (1967). A history of the various initial experiments can be found in Moore and Fung (1979). Following the general procedures discussed above, various empirical forms were developed for the backscatter-to-wind vector relation (Moore and Fung, 1979; Boggs, 1981; Jones et al., 1982; Schroeder et al., 1982a; Woiceshyn et al., 1986). The final choice for SEASAT, discussed in detail below, was a single power law relation with a least squares inversion (Moore and Fung, 1979; Jones et al., 1977). Following the SEASAT mission, the scatterometer model function was tuned through comparison with high quality surface wind data, primarily that collected in the Gulf of Alaska (GOASEX) and Joint Air-Sea Interaction (JASIN) experiments (Jones et al., 1982; Schroeder et al., 1982a, 1982b; Wurtele et al., 1982; Brown, 1983). Some results from these experiments are summarized following a more detailed description of the SEASAT scatterometer.

The SEASAT oceanographic satellite, launched in June 1978, carried several microwave remote sensing instruments, including SASS (Figure 1). The mission of SEASAT was to conduct proof-of-concept experiments for the detection of surface features by remote sensing. These features included surface wind, sea surface temperature, wave height, ocean topography, and sea ice, as well as information on internal waves, atmospheric water vapor, and the marine geoid. The SEASAT mission ended in October 1978 due to a power failure, but the three months of returned data provided sufficient information to meet the original objectives. The satellite flew in an approximately circular orbit with an inclination angle of $108^{\circ}$, at an altitude of $800 \mathrm{~km}$, and with an orbital period of about 101 minutes, thus circling the Earth 14 times a day (Boggs, 1982). The wide swath scatterometer sensor covered $95 \%$ of the ocean every 36 hours (Boggs, 1982). During the first part of its mission SEASAT maintained 
a 17-day repeat cycle (the period before repeating the same ground track), with a minimum spacing between equatorial crossings of $88 \mathrm{~km}$ during that interval. From August 27, 1978 to the end of the mission, SEASAT switched to a three day repeat cycle, with a minimum equatorial crossing separation of $470 \mathrm{~km}$ (Boggs, 1982). The scatterometer operated nearly continously during the mission, returning over 90 days of good information. SASS used four, dual-polarized fan beam antennas, oriented at $45^{\circ}$ and $135^{\circ}$ relative to the satellite track, to get measurements at two orthogonal azimuth angles. The X-shaped illumination pattern of these beams formed a double-sided swath of potential wind-vector data about the satellite subtrack, and a narrow strip of only wind speed information near nadir (Figure 2). The transmitted frequency was $14.6 \mathrm{GHz}$, with a slight shift in the return signal from doppler effects. The antenna beams were electronically subdivided into 15 resolution cells by doppler filtering, using the intersection of the antenna pattern and the lines of constant doppler shift. The final backscatter resolution cell was generated by integrating the received power over a 1.89 second period. The signal processing provided a spatial resolution of approximately $50 \mathrm{~km}$ within the double-sided swaths.

Several sources of instrument error affect the backscatter measurement, $\sigma^{\circ}$, including random errors due to communication noise, uncertainty in spacecraft attitude, and instrument processing, as well as several bias errors (Boggs, 1982). Communication noise is the primary random error and depends on the doppler bandwidth, the signal integration period, and the signal-to-noise ratio (Fischer, 1972). The next most significant source of measurement error comes from uncertainty in spacecraft orientation, and therefore antenna pointing direction. The attitude error encompasses errors in roll, pitch, and yaw, as well as instrument alignment. Boggs (1982) lists values for these various random error sources. Bias errors between the four antennas were estimated from measurements of backscatter over the Amazon rain forest and relatively small corrections were made during sensor data processing. Corrections to $\sigma^{\circ}$ for attenuation through the atmosphere were made for backscatter cells that coincided with measurements made by the Scanning Multichannel Microwave Radiometer (SMMR). This was possible only on the right side of the satellite track, since the SMMR swath 
was one-sided. The lack of attenuation correction is significant primarily for high rain rates, and the anomalous wind values produced under those conditions are easily flagged in most cases. Finally, the $\sigma^{\circ}$ measurements from the integrated "footprints" were screened for anomalous values and for the presence of ice or land, and then input into a geophysical algorithm to obtain the wind vectors.

\section{SASS geophysical algorithm}

The particular geophysical algorithm used for SASS is described completely in Boggs (1982). It computes the $19.5 \mathrm{~m}$ neutral stability wind speed and direction at a particular time and location. Basically, it consists of three components: 1) a cell-pairing process to match forward and aft cell values, 2) a wind-to- $\sigma^{\circ}$ model function, and 3) a least-squares estimator to invert the model function. Prior to the backscatter-to-wind inversion, a complex temporal and spatial reorganization of the backscatter data is needed. The $\sigma^{o}$ cell measurements are spread out across the swath for all particular times and antenna patterns. The wind determination algorithm requires at least two, roughly colocated (in both time and space) $\sigma^{\circ}$ measurements, approximately orthogonal in azimuth. The orthogonality condition requires at least one $\sigma^{\circ}$ measurement from both the forward and aft antenna beams. The data can be grouped either by cell-pairing, in which individual forward and aft cell measurements are matched, or binning, in which all $\sigma^{o}$ measurements within a given area are used to find a single wind solution for that area. Cell pairing was used in the production of the original wind vector data produced from SASS and is described more fully in the next section. Forward and aft beam measurements were paired if they fell within given time and distance separations, with some redundancy of data as some individual cells were paired more than once. The cell-pairing mode was chosen for the initial data production since it yielded the highest resolution wind vector solutions (Boggs, 1982). Subsequent processing (discussed at the end of this section) used the binning mode, with a $50 \mathrm{~km}$ resolution. In both cases, nadir wind solutions (in the narrow strip directly below the satellite) were formed from binned data, without the 
need for orthogonal measurements, due to the lack of directional information at low incidence angles. In each case, the time and location assigned to the wind solution are the centroids of those of the grouped $\sigma^{\circ}$ measurements. Once matched, the paired or binned backscatter measurements are input into the model function, which is the empirical relationship that describes the dependence of $\sigma^{o}$ on the $19.5 \mathrm{~m}$ neutral stability wind. The relation chosen for SASS data can be expressed in logarithmic form as:

$$
\sigma_{\mathrm{db}}^{o}=\mathrm{G}(\theta, \chi, \varepsilon)+\mathrm{H}(\theta, \chi, \varepsilon) \log _{10} \mathrm{U}_{19.5}
$$

where:

$$
\begin{aligned}
& \sigma_{\mathrm{db}}^{o}=\text { backscattered power in decibels } \\
& \theta=\text { incidence angle } \\
& \chi=\text { wind direction relative to radar azimuth angle } \\
& \varepsilon=\text { polarization of incident radiation } \\
& \mathrm{U}_{19.5}=\text { neutral stability wind at } 19.5 \mathrm{~m}(\mathrm{~m} / \mathrm{s})
\end{aligned}
$$

(Boggs, 1982)

The $\sigma^{\circ}$ values have a range of more than $50 \mathrm{db}$ for typical wind speed ranges, so there is a clear advantage to using a logarithmic form (Woiceshyn et al., 1986). It should also be repeated that this particular empirical relationship is an arbitrary choice and does not have a direct physical basis. The incidence angle, $\theta$, is defined at the surface as the acute angle between the antenna look direction and the local surface vertical at a doppler cell center (Figure 3 ). The relative wind azimuth, $\chi$, is the angle between the local horizontal wind direction, $\gamma$, and the radar azimuth angle, $\phi$, measured from north to the projected antenna look direction, at the cell center (Figure 4). The radar azimuth angle can be thought of as the instantaneous azimuthal direction of the antenna beam pattern on the surface (Woiceshyn et al., 1986). So, the model function depends implicitly on the actual wind direction through an explicit relation to the relative wind azimuth angle, $\chi . \quad \chi$ is defined so that the upwind condition, with the antenna pointing directly into the wind, corresponds to $\chi=$ $0^{\circ}$ and the downwind condition to $\chi=180^{\circ}$. The original sensor data processing 
actually used the value of the radar azimuth angle calculated at the sub-satellite point, instead of at the cell center. This introduces a relatively small error that can be neglected in most cases (Boggs, 1982). The wind direction used for SASS processing is defined in the meteorological sense as coming from a given direction, measured clockwise from the north. The empirically derived $G$ and $H$ coefficients are given as coefficient tables for vertical and horizontal polarizations and incremental values of $\theta$ and $\chi$. They are tabulated for $0^{\circ}<\theta<70^{\circ}$ in $2^{\circ}$ steps and $0^{\circ}<\chi<180^{\circ}$ in $10^{\circ}$ steps for both polarizations. A first-order interpolation was used to extract values from the $2^{\circ} \theta$ table, while a second-order interpolation was used with the $10^{\circ} \chi$ table. The dependence of $\mathrm{G}$ and $\mathrm{H}$ on azimuth is approximately $\cos \left(2 \chi^{*}\right)$, where $\chi^{*}$ is the difference between wind direction and radar azimuth, with maxima at $\chi^{*}=0^{\circ}$ and $180^{\circ}$. The harmonic dependence means that the relative wind direction, $\chi$, used in the model function, has a functional range of 180 degrees (Boggs, 1982). The G-H tables were originally determined from aircraft measurements and then modified after several SEASAT validation workshops. The final table was formed by combining two models over different incidence angle ranges (Boggs, 1981; Schroeder et al.,1982a). The selection of parameters and the choice of a relatively simple, logarithmic form with a power law dependence are still subject to considerable debate (Atlas et al., 1986; Woiceshyn et al., 1986), but generally good results and confirmation of basic concepts were obtained from this initial formulation. The model function is a unique relation for the dependence of $\sigma^{\circ}$ on the $19.5 \mathrm{~m}$ neutral stability wind, but the wind retrieval process requires using this relation in the opposite direction. This inversion requires at least two colocated, roughly orthogonally viewed $\sigma^{\circ}$ measurements and is not a unique specification. A weighted least-squares estimation, also referred to as a sumof-squares approach, was used to accomplish the actual inversion (Boggs, 1982; Jones et al.,1982).

One limitation of this $\sigma^{\circ}$-to-wind algorithm is that for a given $\sigma^{\circ}$ grouping there may be up to four possible wind vector solutions, all with approximately the same wind speed, but with widely varying directions. Figure 5 illustrates this for the ideal case of two orthogonal, noise-free, colocated $\sigma^{\circ}$ measurements of the same 
wind. The curves are possible solutions in wind speed (U)-azimuth angle $(\chi)$ space for particular values of backscatter $\left(\sigma^{\circ}\right)$ and incidence angle $\left(\theta_{\mathrm{I}}\right)$. Wind solutions are found at the curve intersections and this case shows the large variation of directions and small range of wind speeds for the different possible solutions. As shown, a $90^{\circ}$ separation in azimuth yields the most distinct intersections. Noisy measurements further complicate this process, since the intersections are then spread over a larger area. These direction aliases cannot be removed without further processing, usually requiring some independent knowledge of the wind field. Future scatterometers will help resolve this ambiguity problem, by adding an additional antenna on each side, reducing the maximum number of solutions to only two, separated by nearly $180^{\circ}$.

\section{SASS performance evaluations}

Evaluations of the final SASS wind retrieval algorithms initially indicated that it met the required specifications of $\pm 2 \mathrm{~m} / \mathrm{s}$ in wind speed and $\pm 20^{\circ}$ in wind direction on a synoptic scale. The final results from the SASS workshops were rms differences of $1.3 \mathrm{~m} / \mathrm{s}$ for a range of 4 to $26 \mathrm{~m} / \mathrm{s}$, and $16^{\circ}$ for a $0^{\circ}$-to- $360^{\circ}$ range (Lame and Born, 1982; Jones et al., 1982). However, these rms values were calculated for a restricted data set that was limited to the particular wind speed range and geographical area of the conventional wind data used for tuning and validation. These statistics do not always accurately reflect performance for other subsets of the data, as discussed in detail by Woiceshyn et al. (1986). Sources of error for the SASS generated winds exist both in the geophysical interpretation of the radar signal and in factors that may contaminate the signal, such as rain, sea surface temperature, and radar polarization. Many of these questions were addressed in the scattering theory discussion. An additional point is that the inherent spatial averaging of each SASS footprint may smooth out variations of the small scale turbulent winds (up to $10 \mathrm{~m} / \mathrm{s}$ ) embedded in the footprint area (Brown, 1986). The spatial smoothing may be especially significant for small, intense features, such as thunderstorms. Strong circulation features, such as fronts, cyclones, and hurricanes, also pose a problem, since they generally 
violate the steady state conditions assumed in the $\sigma^{\circ}$-to- $U$ algorithms. Differences in wind retrieval system behavior across a front are evident in the changed character of the scatterometer signal across the front (Brown, 1983). Adjustments to the wind retrieval system are needed to more fully account for these flow features, most likely as parameterizations that can be incorporated into the steady state algorithms (Brown, 1986).

Various evaluations of the SASS data have identified problems with different aspects of the wind retrieval algorithms. Woiceshyn et al. (1986) use intercomparisons of horizontally and vertically polarized SASS data and comparisons between in situ and SASS winds to demonstrate the inadequacy of the power law relation at all observed wind speeds and incidence angles. This stems from the tuning of the data to the limited range of conditions observed in the "surface truth" experiments and to the inability of a single power law to model the difference in surface roughness over two distinct wind regimes. Woiceshyn et al. (1986) discuss other problems, including the inaccuracy of the SASS winds at low wind speeds. The combination of a sum-ofsquares inversion technique and the signal from noise subtraction process may yield negative backscatter values for light winds (Pierson et al., 1986). Using a power law assumption with this inversion process means that the negative values must be discarded, causing a considerable loss of information at lower wind speeds. A better option may be to use a maximum likelihood estimation inversion that will make use of all the data (Pierson et al., 1986). Preliminary work on a new scatterometer wind extraction system that removes many of these biases in the SASS model is presented in Woiceshyn et al. (1984). A careful statistical analysis of the original SASS winds by Wentz et al. $(1984,1986)$ also revealed several systematic errors in the SASS data. They found that horizontally polarized backscatter signals yielded winds that were biased high relative to those from vertically polarized backscatter returns, a crossswath wind gradient error was introduced from an incorrect $\sigma^{\circ}$ vs incidence angle relation, low signal-to-noise ratio $\sigma^{\circ}$ values were discarded - so low winds were biased high and data gaps existed for areas of light winds, and the SASS winds were biased high by approximately $1 \mathrm{~m} / \mathrm{s}$ relative to conventional winds. Some of these errors are 
similar to those discussed by Woiceshyn et al. (1986), but Wentz (1986) retains the power law relation and corrects the model coefficients, rather than choosing another form for the model function. Finally, the question of directional ambiguity in the wind vector solutions has received considerable attention (Wurtele et al., 1982; Peteherych et al., 1984). Several alternatives to the SASS algorithm method have been developed (Hofman, 1982, 1984; Gohil and Pandey, 1985; Woiceshyn et al., 1986; Brown, 1986), and the design of future scatterometers will considerably reduce this alias problem.

\section{SASS data reprocessing}

The original SASS wind data, processed with the model function and cell-pairing method described above, is available on Geophysical Data Record (GDR) tapes located at the Jet Propulsion Laboratory (JPL). The alias problem and other errors discovered in the initial GDR processing, mentioned briefly above, motivated a reprocessing of the entire sensor data set. Wentz et al. (1986) reprocessed the entire 96-day data set, binning the backscatter values into $50 \mathrm{~km}$ cells oriented perpendicular to the satellite subtrack. These combined $\sigma^{\circ}$ measurements were then inverted to obtain the wind vectors, on both 50 and $100 \mathrm{~km}$ grids. The winds were retrieved with an improved $\sigma^{\circ}$-to-wind model, based on an assumed Rayleigh wind distribution, rather than tuning to in situ measurements. The model was designed to minimize the systematic errors dependent on polarization or incidence angle identified earlier, and used a single power law relationship, except for an adjustment for the higher nadir winds. Based on climatology, the winds were assumed to be Rayleigh distributed about a mean of $7.4 \mathrm{~m} / \mathrm{s}$ (Wentz et al., 1984). This statistical approach has several advantages over a tuning approach, including the ability to use all the satellite measurements, rather than just those matched with a limited, high-quality conventional data set, and elimination of the problem of matching the different temporal and spatial scales of satellite and conventional data. The pre-averaging of the backscatter measurements across larger cells increases the signal-to-noise ratio (SNR) for the final $\sigma^{o}$ value, which is especially useful at low wind speeds. A typical cell in the middle 
of the swath contained four $\sigma^{\circ}$ measurements from each antenna, and SNR increased by a factor of two (Wentz et al., 1986). These reprocessed winds show more consistency and lower residual errors than the original SASS winds in intercomparisons over different polarizations and incidence angles. A comparison to buoy winds showed good general agreement in wind speed, with a $1.6 \mathrm{~m} / \mathrm{s} \mathrm{rms}$ difference and $-0.1 \mathrm{~m} / \mathrm{s}$ bias, supporting the statistical assumptions made in deriving the new model (Wentz et al., 1986).

The directional ambiguity in the SASS winds was removed by several different methods, both subjective and objective, for various subsets of the original data. Peteherych et al., (1984) developed a subjective dealiasing method and produced a global, 15-day (6-20 September 1978) set of dealiased SASS winds. Their method is based on procedures described by Wurtele et al., (1982) and Baker et al., (1984), where meteorological analysis and pattern recognition are used to select an alias. Rather than using subjective meteorological analysis to dealias the data, alternative objective techniques have been developed, including those of Hoffman (1982, 1984), Baker et al., (1984), and Yu and McPherson (1984). Atlas et al., (1987), used a modified version of the model of Baker et al., (1984) to objectively dealias the complete 96-day set of reprocessed, $100 \mathrm{~km}$ resolution, multiple-solution vectors. Atlas et al., (1987) employed the Goddard Laboratory for Atmospheres (GLA) analysis/forecast system in an iterative, three-pass procedure, combining conventional data and previously analyzed SASS winds as a first-guess field. These unambiguous SASS wind vectors are subsequently identified as Atlas winds in this paper, to distinguish them from the original GDR winds. They form the principal set of scatterometer winds to be evaluated and compared with conventional wind data in this study. 


\section{Data description and handling}

The initial data set obtained was a subset of the GDR data, supplied by NASA Ocean Data Systems (NODS) at JPL. Figure 6 shows a swath of these winds from SEASAT revolution 184. The alias problem discussed above limited its usefulness, and when the Atlas data set became available it was used, rather than the GDR data, for all subsequent evaluations. As mentioned in Section 3, the Atlas SASS winds are referred to as Atlas winds, in order to distinguish them from the original GDR data and from other sets of SASS wind data. In the preliminary analysis, we removed the directional ambiguity for selected GDR winds subjectively, by comparison of the plotted GDR wind vectors to synoptic weather and pressure maps. The SASS solution in closest agreement with the conventionally derived wind field was selected wherever possible, while those cases where an unambigous solution was not obvious were dropped from further consideration. Due to uncertainties imposed by this rough dealiasing scheme, the GDR winds were most useful in an initial screening of the Atlas winds, rather than as input for further analysis. After basic pattern agreement was established, these winds were most useful in ensuring the proper reading, transfer, and display of Atlas wind vector data. This study concentrates on a one-month subset of the full 96-day record, from 12 August to 10 September 1978, over a portion of the western North Atlantic, from $20-30^{\circ} \mathrm{N}$ and $40-80^{\circ} \mathrm{W}$. The Atlas data is regularly spaced along the satellite subtrack, but it is irregularly spaced on a latitude-longitude grid, and the nature of satellite orbit ground coverage means that data samples in a given area are widely separated in both time and space. Typically, two passes cross the study area, separated by approximately 100 minutes and $2500 \mathrm{~km}$, followed 12 hours later by another two passes, offset in space from the first pair.

Since the primary purpose was to compare scatterometer winds to a conventional wind field, a readily available, suitable conventional wind field was needed. The 6-hour synoptic surface wind analyses provided by the Fleet Numerical Oceanographic Center (FNOC) in Monterey, CA, through the National Center for Atmospheric Research (NCAR), were selected. These winds are generated by combining ship and buoy wind 
reports with pressure-derived winds and are inherently smoothed to some extent on larger pressure field scales. A subset of the analyses was extracted, covering the same western North Atlantic area as the scatterometer data, interpolated to a $1^{\circ}$ latitude by $1^{\circ}$ longitude grid. In addition to this wind field data, synoptic weather and pressure charts were also employed. These small-scale, large-area facsimile charts are quite crude, but provide a source of qualitative information for comparison to both the Atlas and FNOC fields.

An important factor in any comparison between satellite and conventional measurements is the different temporal and spatial averaging inherent in each. The scatterometer footprint is at the shorter end of the mesoscale range, a measurement averaged over roughly $50 \mathrm{~km}$ in space and nearly instantaneous in time, but sampled at widely separated intervals of both space and time. Ship and buoy wind measurements are point measurements in space, and may be averaged over an arbitrary time period, especially in the case of the buoy winds. Conventional, pressure-derived surface wind fields have larger, synoptic scales, comparable to those of the scatterometer swath $(500$ to $1000 \mathrm{~km})$. These differing scales are important to a proper interpretation of the differences between the various data (Pierson, 1983; Stewart, 1985).

So the available data are the GDR winds, the Atlas winds, the FNOC winds, and the synoptic weather and pressure maps. The primary comparison is between the Atlas and FNOC winds, both between vector fields and in a broader, statistical sense, with the weather maps used mostly for initial screening and evaluation. This screening included verifying the general flow patterns and the location of significant meteorological features. The Atlas-FNOC wind comparison was hindered immediately by the irregular spacing of the Atlas data (relative to a latitude-longitude grid). Boxed mean differences were calculated for some areas, by calculating component means for each field for a series of areas, then finding the differences between the means in these boxes. These have limited usefulness, especially since the resolution of the AtlasFNOC difference field generated in this manner is very poor, so further processing of the scatterometer winds was necessary to regrid the field. To obtain regularly gridded 
scatterometer data, objective mapping techniques were employed, concentrating on spatial mapping.

Objective mapping procedure

Objective mapping is basically an interpolation scheme that incorporates knowledge of the field statistics and data locations in construction of an informed estimate of the field at desired locations. A major advantage of the process is that it provides an estimate of the error field at the same time, yielding an objective measure of how well the mapping does at different points. The formulation used here follows that of Bretherton, Davis, and Fandry (1976), and most details of the actual mapping procedure are omitted, except for the form of the data covariance matrix, the general form and particular choice of which are described briefly below. Input of field information is made through choices of the form and scales of the data covariance matrix. This matrix, $A_{r s}$, has the general form:

$$
A_{r s}=F\left(x_{r}-x_{s}\right)+E \delta_{r s}
$$

where:

$$
\begin{aligned}
& A_{r s}=\text { covariance matrix of all pairs of observations, } r \text { and } s \\
& F\left(x_{r}-x_{8}\right)=\text { covariance as a function of spatial separation } \\
& E=\text { variance due to measurement noise }
\end{aligned}
$$

The covariance function, $F(\vec{x})$, where $\vec{x}$ is the spatial separation in $x$ and $y$, is used to form the covariance matrix between of pairs of individual data measurements. After experimenting with various forms of this covariance function, a simple gaussian form was chosen:

$$
\mathrm{F}(\overrightarrow{\mathrm{x}})=\sigma_{\mathrm{S}}^{2} \exp \left(\frac{-\mathrm{x}^{2}-\mathrm{y}^{2}}{\mathrm{R}_{\mathrm{S}}^{2}}\right)+\sigma_{\mathrm{N}}^{2} \exp \left(\frac{-\mathrm{x}^{2}-\mathrm{y}^{2}}{\mathrm{R}_{\mathrm{N}}^{2}}\right)
$$

where:

$$
\begin{aligned}
& F(\vec{x})=\text { covariance function } \\
& \sigma_{\mathrm{S}}^{2}=\text { signal variance }
\end{aligned}
$$




$$
\begin{aligned}
& \mathrm{R}_{\mathrm{S}}=\text { signal scale (for both } \mathrm{x} \text { and } \mathrm{y} \text { in this case) } \\
& \sigma_{\mathrm{N}}^{2}=\text { "noise" variance due to small scale signal } \\
& \mathrm{R}_{\mathrm{N}}=\text { "noise" scale }
\end{aligned}
$$

Both of these matrices, $A_{r s}$ and $F(\vec{x})$, are dependent only on the position of the observations, so they are constant for different realizations of the data field, unless one expects the statistics of the data field to change with time. In that case, the scales of the covariance function must be adjusted according to the expected statistical evolution of the data field. The choices of scales and variances used in this formulation depend on the expected allocation of the field information into signal and noise. In this case, the "noise" is actually real information, usually at the smaller scales, that is arbitrarily designated as noise. This division depends mostly on what scales are present in the data and on what scales we are interested in studying. Additional scales can be incorporated explicitly in the covariance function as well, each with a similar form to that shown above. The relative weight of each different scale is determined by the magnitude of the variance, also known as the amplitude function. Expected instrument noise is incorporated in the noise level, E, representing the contribution of random noise to the variance at each observation point. The $A_{r s}$ covariance matrix is inverted in the mapping process, and a small amount of measurement noise is usually needed to keep the process mathematically stable, even if "perfect" data are available.

An advantage of objective mapping is that it yields a measure of the error, due both to the procedure and to the statistical assumptions made about the data. The magnitude and structure of this error variance can be used to assess the impact of various imposed conditions on the mapping process. Some definitions of terms used to describe the accuracy of the mapping are needed at this point. For the case of unit signal variance, in which the error maps are non-dimensional, useful mapping is defined as areas with error variance $\leq 0.6$ and accurate mapping is defined as areas with error variance $\leq 0.3$. The signal spatial scale (or scales), $R_{S}$, has the greatest impact on the estimate field as a whole, both through the need for it to be consistent with the actual scales of the data and in its relation to data 
spacing. The imposed signal scale must be reasonably close to those of the original field for the mapping process to work. The area of accurate mapping is considerably increased when the signal scale is larger than the data spacing (Bretherton, Davis, and Fandry, 1976). Varying the noise scale, $R_{N}$, has an impact that seems confined mostly to the vicinity of each data point. Choosing a vanishingly small noise scale causes the small-scale noise to exert an effect similar to measurement noise over much of the field, except exactly at the data locations. At these points it yields a conservative error estimate (Bretherton, Davis, and Fandry, 1976). Choosing $\sigma_{\mathrm{N}}^{2}=0$, thereby assuming that the "noise" from the small-scale signal is not significant, does not change the area of useful mapping, compared to a choice of a small $\left(\sigma_{\mathrm{N}}^{2} \simeq 0.1\right)$ noise variance, when an infinitesimal noise scale $\left(R_{N}=0\right)$ is used. However, this vanishing noise does increase the area of accurate mapping (Bretherton, Davis, and Fandry, 1976). These general considerations were taken into account in the initial selection of the covariance function, with the final choice decided by how well the resulting estimate field reproduced the original winds.

To avoid the complication of mapping vector quantities, the $U$ and $V$ components were mapped separately as independent scalar properties. Several combinations of scales and variances were tested, with an eye towards finding the simplest formulation that would accurately reproduce the wind field. The final selection was:

$$
F(\vec{x})=1.0 \exp \left[-\left(\frac{x}{500 \mathrm{~km}}\right)^{2}-\left(\frac{\mathrm{y}}{500 \mathrm{~km}}\right)^{2}\right]
$$

The choice of a $500 \mathrm{~km}$ signal scale smooths the original field slightly, depending on the amount of variation at yet smaller scales. The choice of $\sigma_{N}^{2}=0$ reflects the fact that we expect the small-scale signal variation to not contribute significantly to the total data covariance. After experimenting with different values of the noise variance, a simple choice of a constant $E=0.1$ was made, so measurement noise is incorporated along the diagonal of the covariance matriz, $\mathbf{A}_{\mathbf{r s}}$. This value is somewhat arbitrary, since it is difficult to precisely determine the measurement, or instrument, noise of scatterometer data. Instrument error is a combination of many different SASS sensor 
data errors, and also includes potential errors introduced during reprocessing of the backscatter data. In initial experiments, this choice of a reasonably conservative instrument noise variance produced good results, so it was used in all subsequent mapping. If the covariances imposed on the estimated field are inconsistent with those of the actual data, the mapped field can vary considerably from the original field. More severe inconsistency in the imposed conditions can introduce ill-conditioned matrices, resulting in a breakdown of the mapping process, either through a failure of the matrix inversion, or through the production of worthless estimates. The extent to which the estimated field reproduces the original is a good indication of whether the imposed scales and variances are appropriate. Ideally, the difference between the estimated and original fields at any point should be close to the imposed noise at that point. The estimate formed from the simple covariance function and variances given above is a good representation of the original data (a more detailed comparison is given below), so those choices were employed in all subsequent mapping.

The chosen mapping procedure is one that includes an estimate of the mean and may result in error variances greater than the signal variance (greater than unity in the non-dimensional case) in some cases. The mapping process was initially evaluated by mapping two revolutions, 744 and 830 , that contained a large number of wind vectors, and then overlaying vector plots of the original and mapped data and examining the structure of the error maps. Overlaying vector plots showed the agreement between both the closely colocated vectors and the general circulation patterns of each field. In regions of abundant data, the mapping closely reproduceed the original field in both respects, with a small amount of smoothing of markedly anomalous vectors, and in areas where winds change rapidly over short distances. The latter is particularly evident in regions of light and variable winds when no well-defined circulation pattern is present. A notable feature of these initial maps is the relatively poor performance in data-sparse corners of the estimate grid that require significant extrapolation from interior points. These extrapolated border values are noticeably worse than those between the swaths, which is an expected result since the extrapolation is one-sided, and this emphasizes the need to map only over areas with reasonable amounts of 
data. Lowest error variance values are found where data is densest, and the doubleswath pattern of the data field is quite evident in the associated error maps (Figures 7 and 8).

Evaluations of this type, made on the initial runs, demonstrated that the chosen mapping procedure worked well and that the chosen covariance function was appropriate. However, the spatial distribution of the data presented an entirely new problem. Mapping the diagonal satellite tracks of entire revolutions in the study area produced large regions of high error variance, with little useful information, on either side of the satellite swath. In addition, the fairly large numbers of wind vectors present in each revolution are computationally expensive to map, since the objective mapping routine requires inverting a square matrix with a dimension equal to the number of data. Thus, the need to discard large amounts of information from the large-area mappings is even less acceptable. The solution chosen here was to map successive boxes along the path of each satellite revolution. From one to four boxes were needed for each revolution, depending on the length of the track (Figure 9), and data input for each box included all the vectors within one correlation distance $(500 \mathrm{~km})$ of each edge. One drawback to this process is that by using smaller grids we lose low wave number information, especially in the meridional direction, since the swaths are aligned more north-south than east-west. However, this loss should not be significant since we expect the smaller, synoptic scale $(\sim 500 \mathrm{~km})$ to dominate most of the signal. Synoptic scale dominance is particularly true in the meridional direction, due to the rather strong dependence of weather patterns on latitude and the tendency for weather systems to move in a zonal direction. It should be noted that low wave number information is also missing in the zonal direction, since the swaths are not wide enough to resolve it. The lack of low wave number information in the zonal direction cannot be corrected without combining data from several revolutions, and in that case, the large temporal and spatial separations involved introduce yet further complications. For this study, the loss of low wave number information was accepted as a necessary trade-off for the use of a simpler, spatial mapping. 
As before, the mapped data was checked by comparison to input scatterometer data through vector plot overlays and error variance plots, and through comparing statistics of the original and estimated fields. This time a more detailed comparison was done, using objective maps made for all the revolutions on each of three separate days, spread through the data set. The scatterometer revolutions from days 230,236 and 248 each contained swaths with large numbers of winds vectors, as well as both well-defined circulation patterns and regions of more variable winds. These features facilitated the subsequent comparison to the mapped wind field. By overlaying vector plots, we can see the agreement between both the closely colocated vectors and the general circulation patterns. The circulation patterns, especially those around the strong low pressure systems in the north, are closely reproduced, agreeing in the inferred location of the low pressure center and associated fronts, and in the direction and magnitude of overlapping vectors. Revolution 830 , on day 236 , is a good example of this (Figures 7 and 9). Some of the highest magnitude vectors in the original field are not picked up in the estimate field, especially in cases where there is one anomalously high vector in a field of generally lower magnitude. Likewise, some of the lighter winds are overestimated in the mapped field, particularly in the case when they are surrounded by or border on an area of higher magnitude winds. Both of these reflect the slight smoothing of the mapping process, which tends to reduce anomalous vectors embedded in a more uniform field. More isolated vectors are generally closely reproduced in the mapping, since they hold relatively more weight in a data-sparse area, and they are then joined by extrapolation to areas with more input information. This worked well for vectors that fit reasonably well into the overall pattern and did not have extreme spatial separations, which was usually the case for isolated points in this data. In any case, the need for excessively large extrapolation was minimized, since care was taken to map over regions of denser data. Differences in closely colocated vectors from the two fields were most evident in regions of light and variable winds, where the winds change rapidly and more randomly over relatively short distances. The wind magnitudes tend to be the same, but the directions may vary considerably, up to 180 degrees in a few cases. However, since this variation is 
typical of those areas in the first place, and since the wind magnitudes are small, the impact on the final results is minimized. Statistics of the two fields were similar for these initial cases, also confirming our choice of covariance matrix. Statistics for each field are given for the complete data set in Section 5 . Overall, the mapping procedure is effective at accurately reproducing the original field; in the general circulation patterns, for overlapping vectors, and in a broader, statistical sense. 


\section{Results and discussion}

Prior to the detailed intercomparison of the FNOC and Atlas wind fields, they were initially compared to matching synoptic weather charts to obtain a rough subjective estimate of their quality. This comparison was used to verify basic flow patterns, as well as the location of and circulation around major weather features. It was a means to identify gross errors and it also helped to resolve some computer plotting questions. To the extent possible, given the coarse nature of the weather maps, both wind fields compare well to the flow patterns evident in the weather maps. In particular, the series of strong low pressure systems that tracked across the northern section of the study area are closely reproduced in the Atlas and FNOC winds. The wind field inferred locations of the low pressure centers are sometimes displaced slightly $(\leq 200 \mathrm{~km}$ ) from those of the meteorological analyses, especially for the Atlas winds. However, no extreme displacements are noted, and in each case the difference is well within the coarse resolution limits of the weather maps. The broader circulation associated with these low pressure systems, as reflected in the Atlas and FNOC wind patterns, is consistent for each with the isobars and frontal systems of the meteorological analyses. This consistency is true both for the spatial patterns observed at a particular time, and for the temporal evolution of the patterns across several synoptic analysis periods. The major difference stems from the greater resolution and higher variability in the two wind fields, which contain small-scale features that are smoothed out of the weather maps. For the Atlas winds, some of the differences can also be attributed to the offset in the times of the satellite passes from the 6-hourly synoptic analysis times. This difference in time can be up to 3 hours, but is generally less than 1 hour for this data. The strongest features during the study period were Hurricane Ella and Tropical Storm Flossie, and both showed up clearly and in good agreement with the meteorological analyses in the Atlas and FNOC winds.

The comparison between the mapped scatterometer and FNOC winds was accomplished in several ways, both qualitative and quantitative. Atlas data from a particular satellite revolution were matched to colocated FNOC data at the synoptic 
time closest to the revolution time. Overlaying plots of the two fields provided a good qualitative look at their differences. Basically, the fields appear very similar, especially across areas with little spatial variation in the wind. Significant differences in both magnitude and direction are observed in wind vectors from the two fields in the vicinity of strong weather features. Some of these differences can be accounted for by the offset in time between the two fields, which may be up to three hours. Also, the sampling pattern of the scatterometer did not always provide complete spatial coverage of important features, and filling in with mapped values at the margin of available data gives uncertain results, due to the high error variance in the mapping there. The difficulty posed by these high variance areas was minimized by mapping only within the extent of available data as much as possible. Another explanation of the observed differences between the two fields is the higher spatial resolution and therefore greater inherent variability of the Atlas winds. Even though there is some smoothing of the scatterometer winds, both in the data generation and objective mapping stages, it is still on a scale less than that used to generate the FNOC winds. The impact of this greater variability is expected to be much more near areas of intense, smaller-scale circulation, and the observed differences in the two wind fields reflect this.

Quantitative comparison of the Atlas and FNOC wind vectors is accomplished in several ways: 1) statistical descriptions of each field, 2) differences in the mean values within latitude-longitude boxes, 3) scatter plots of wind speed and direction for each field, and 4) statistical descriptions of the difference field (Atlas-FNOC). Initial evaluations include the ungridded as well as the mapped Atlas winds, but subsequent calculations require colocated vectors and are only done for the mapped Atlas and FNOC winds. Most calculations were done on the east and north components of the wind, but some vector statistics are also shown. The various quantities are computed for the instantaneous vector field "snapshots" and also for one-day and three-day averaging periods. In addition, the statistics are also subsequently grouped by latitude to examine its impact, since weather patterns, and therefore wind, tend to group in zonal bands. Most of the comparisons done in this study concentrate on the wind velocity, since that is the data available, rather than on the wind stress, though it is 
recognized that stress is a better representation of the driving force provided by the wind. However, the conversion from velocity to stress is not trivial, especially over larger areas and longer periods of time, and introduces additional uncertainties due to factors such the formulation of the drag coefficient. Also, the stress values are quite small and this can cause problems in the difference calculations. For these reasons, the bulk of the statistics are calculated for the wind velocity, either in component or vector form. In addition to these velocity calculations, the wind stress curl is calculated for the Atlas and FNOC wind fields, and examined for significant and consistent differences betweem them. Since this parameter is so crucial to ocean circulation forcing, we found it useful to find and compare the values from the two fields, recognizing the limitations on stress calculations discussed above. 
Field statistics

Three separate wind fields are examined in this study, the ungridded Atlas SASS winds, the objectively mapped Atlas winds (derived from the first), and the FNOC combined pressure-derived and buoy and ship report winds. Descriptions of the individual wind fields are provided in various forms, qualitative and quantitative. Representative vector plots, mean and standard deviation for data within individual areas (matching the objective mapping grid), and the mean and standard deviation of all data on a particular day are given for all three fields. In addition, for the mapped Atlas and FNOC fields, mean speed and vector statistics, daily averages grouped by latitude, and three-day averages are shown.

\section{Ungridded Atlas winds}

For the ungridded Atlas winds, Table 1 shows the $U$ and $V$ component statistics for individual areas in each revolution, including mean, variance, weighted variance, standard deviation, and number of data. In this and following tables, the variance is simply the sample variance and the weighted variance accounts for the effect of varying wind speeds on variance values and is normalized by the rms value:

$$
\sigma_{\mathrm{w}}^{2}=\frac{\left\langle\left(\mathrm{U}_{\mathrm{i}}-\overline{\mathrm{U}_{\mathrm{i}}}\right)^{2}\right\rangle}{\left\langle\mathrm{U}_{\mathrm{i}}^{2}\right\rangle}
$$

The data used includes all those vectors within the corresponding mapping grid as well as within one correlation length (that used in the mapping) of any grid edge. This is the same data that was used as input to the objective mapping program. Figure 10 shows the distribution of the component means and standard deviations graphically. Areas of high wind speed, such as $715 \mathrm{U} 1$ and $735 \mathrm{U1}$ (where U1 indicates a particular box along the satellite revolution), or high variance relative to wind speed, such as $715 \mathrm{~V} 1$ and $779 \mathrm{U} 2$, can be associated with meteorological features present near those areas, as shown by synoptic weather maps. Those areas with high mean wind speed and significant variance, especially $836 \mathrm{U} 2$ and V2 and $1008 \mathrm{U} 2$ and V2, straddle circulation about well-developed low pressure systems, reflecting the strength of the 
system and the sharp variation in wind direction across fronts and around the low. Areas that include a single front, like 7151 , show a large mean and lower variance in one direction ( $U$ in this case), and a low mean with much larger variance in the other direction. Across this particular front, while the winds remain predominantly easterly, the north-south direction varies up to $180^{\circ}$, driving down the mean and increasing the variance in the $\mathrm{V}$ component. For the entire data set, the mean $\mathrm{U}$ values tend to be greater than the corresponding $V$ values on average, roughly two-thirds of the time, but the weighted variances are split nearly evenly in relative size for the two components.

Statistics for the $\mathrm{U}$ and $\mathrm{V}$ components of the ungridded Atlas winds, averaged over one-day periods rather than just instantaneous "snapshots", are listed in Table 2. The mean magnitudes are evenly split, with neither $\mathrm{U}$ or $\mathrm{V}$ relatively larger on average, and the weighted variances are fairly even, with $\sigma_{u}^{2}>\sigma_{v}^{2} 17$ out of 29 times (59\%). The daily means are lower than those for the individual areas in Table 1, and the variances increase, with the standard deviation now greater than the mean in each case. The lower mean values and higher variances are consistent with what we expect, since over the course of one day the samples are taken from widely spaced areas, usually from 2 or 3 revolutions, and the winds can vary greatly within those space and time scales.

\section{Mapped Atlas winds}

The distribution of $\mathrm{U}$ and $\mathrm{V}$ components, and wind speed and direction from all the mapped Atlas vectors in this data set are shown in Figure 11. The $U$ component is evenly divided between positive (westerly wind) and negative, while the $V$ component is more frequently positive (southerly wind). The distribution of magnitudes coarsely reflects the Rayleigh distribution expected of the original Atlas data, providing more confirmation of the objective mapping process. The wind direction bins indicate the compass direction the wind is blowing toward, for example, $0^{\circ}$ is a southerly (or northward) wind. This distribution also shows the slightly more frequent occurrence of southerly winds. Various statistics for the $U$ and $V$ components in individual 
boxes (within revolutions) for the objectively mapped Atlas SASS winds are listed in Table 3 and shown graphically in Figure 12. It should be noted that these cannot be directly compared to the corresponding values for the ungridded winds, since those were calculated over slightly larger areas and included more data. Again, mean U magnitudes tend to be larger, with $|U|>|V| 68$ out of 117 times (59\%). The relative values of the weighted component variances are generally close, and $\sigma_{u}^{2}>\sigma_{v}^{2} 51$ out of 117 times (44\%). Compared to the values for the ungridded winds, the weighted variances of the mapped winds tend to be slightly lower, with $\sigma_{\mathrm{un}}^{2}>\sigma_{\text {map }}^{2} 187$ out of 234 times (80\%), including 101 of 117 (86\%) for $U$ and 86 of 117 (74\%) for V. This trend is expected since the objective mapping process smooths the data field to some extent. Table 4 presents the statistics for the $U$ and $V$ components of the mapped SASS winds, averaged over one day periods. The means are reduced relative to the individual "snapshot" values, and the variances increase to the point where the standard deviation is greater than the mean in most, though not all, cases. The component magnitudes are evenly split in relative size, while the weighted variances show some difference between components, with $\sigma_{\mathrm{u}}^{2}>\sigma_{\mathrm{v}}^{2} 19$ out of 29 times (66\%). The greater variance of the zonal wind, on average, reflects the slightly greater range of speeds associated with it, as compared to the meridional wind. In North Atlantic midlatitudes, one expects the average zonal wind to be larger than the average meridional wind (Leetmaa and Bunker, 1978). Component statistics, again averaged over one day periods and now also grouped into $10^{\circ}$ latitude bins are shown in Table 5. Tables 6 and 7 list one-day and three-day average vector statistics, including the mean speed, the speed and vector variance and standard deviation, and the percentage of the vectors that fall in each of four direction bins. The standard deviation of the wind vector is defined as:

$$
\sigma_{\text {vec }}=\left(\frac{1}{N-1} \sum_{i=1}^{N}\left[\left(u_{i}-\bar{u}\right)^{2}+\left(v_{i}-\bar{v}\right)^{2}\right]\right)^{\frac{1}{2}}
$$

while the standard deviation of the mean wind speed is defined as:

$$
\sigma_{\mathrm{spd}}=\left(\frac{1}{N-1} \sum_{i=1}^{N}\left[\left(|U|_{i}-\overline{(U \mid)^{2}}\right]\right)^{\frac{1}{2}}\right.
$$


where: $|\mathrm{U}|=\left(\mathrm{u}^{2}+\mathrm{v}^{2}\right)^{\frac{1}{2}}$

The mean speed and vector deviations are quite different, with $\sigma_{\text {spd }}$ smaller and much more uniform across a given area than $\sigma_{\mathrm{vec}}$. In this case the directions are given in the oceanographic sense of going toward a particular direction. The bins each include $90^{\circ}$ of arc, centered on the given direction. For example, the north bin includes $315^{\circ}<\theta \leq 45^{\circ}$, where $\theta$ is the direction that the wind is blowing toward.

\section{FNOC winds}

The distributions of the $\mathrm{U}$ and $\mathrm{V}$ components, and wind speed and direction from all the FNOC wind vectors are shown in Figure 13. As with the Atlas winds, the U component distribution is divided evenly between positive and negative values and the $\mathrm{V}$ component is more frequently positive, indicating southerly winds. The magnitude distribution differs from that of the Atlas winds, with more winds at the lower wind speeds. However, there are several FNOC values in the highest speed range (25-30 $\mathrm{m} / \mathrm{s}$ ), while there are no Atlas values of that magnitude. The direction distribution shows the tendency for the FNOC winds to be southerly, to an even greater extent than the Atlas winds. Statistics for the $U$ and $V$ components in individual areas for the FNOC wind field are listed in Table 8, and shown graphically in Figure 14. The chosen times are those closest to the time of each satellite pass, and given the 6-hour spacing of the FNOC analyses, may be up to 3 hours different from the time of the satellite pass. Most times were much closer than this upper limit, and in all cases, the best FNOC match was at either the $00 \mathrm{Z}$ or $12 \mathrm{Z}$ analysis times. The mean $U$ magnitude is larger than the mean V magnitude slightly more times, 88 of 144 (61\%), with one case where both are the same, and a slightly greater proportion of the $\mathrm{V}$ component winds have higher variances than the corresponding $U$ component, 83 of $145(57 \%)$. The trend observed in the two sets of Atlas winds, of higher means in one component relative to the other being associated with lower relative variances in that same component, is also seen in the FNOC data. Again, we expect winds 
of greater variability to have their means reduced, when cases of both positive and negative component wind speeds are summed.

Table 9 presents the one-day average statistics for the $\mathrm{U}$ and $\mathrm{V}$ components of the FNOC winds. As was true for the scatterometer winds, the daily means are lower than the earlier means for individual areas, and the variances are relatively larger, with standard deviations greater than the mean values in most cases. The mean $\mathrm{U}$ value tends to be larger than the corresponding $\mathrm{V}$ mean value more of the time, with $|U|>|V|, 17$ of 29 times (59\%). The weighted variance of the $U$ component is also greater than that of the $\mathrm{V}$ component more frequently, with $\sigma_{\mathrm{u}}^{2}>\sigma_{\mathrm{v}}^{2} 19$ of 29 times $(66 \%)$. The greater variance in the zonal wind can again be associated with the wider speed range of the zonal $(-2.5$ to $3.7 \mathrm{~m} / \mathrm{s})$ compared to the meridional $(-0.8$ to $3.8 \mathrm{~m} / \mathrm{s}$ ) winds. The mean daily meridional winds are mostly positive, indicating southerly winds, which is the expected average flow in this area at this time of year. This behavior parallels what is observed in the scatterometer winds as well. Table 10 lists statistics for the component winds, averaged over one day periods and grouped into $10^{\circ}$ latitude bins. Tables 11 and 12 list one-day and three-day average vector statistics, including the percentage of the vectors within each averaging period that fall in each of four direction bins. Again, the directions indicate wind blowing toward a particular direction. 
Boxed mean differences

Prior to regridding the scatterometer winds, a preliminary evaluation of the difference between the scatterometer and FNOC winds was made by calculating the difference between the mean $\mathrm{U}$ and $\mathrm{V}$ components over $10^{\circ}$ latitude by $10^{\circ}$ longitude boxes. This was done for a limited subsection of the data, three separate days, in order to get a rough idea of the magnitude of the difference and possibly any obvious bias or structure. To increase the amount of data used in calculating the mean values and to provide adequate spatial coverage, daily averages were computed. This was done simply by using all the FNOC data from the $00 \mathrm{Z}$ and $12 \mathrm{Z}$, as well as $00 \mathrm{Z}$ of the following day, synoptic analysis times, and the scatterometer data that fell within three hours of those times. Data from three separate days, 18 and 24 August and 5 September (Julian days 230, 236, and 248 respectively), were investigated. For day 230 , data from SEASAT revolutions $744,750,751$, and 758 were used; for day 236 , from revolutions $830,836,837$, and 844 ; and for day 248 , from revolutions 1002 , $1003,1008,1009,1016$. Tables 13,14 , and 15 list the $\Delta U$ and $\Delta V$ mean and standard deviation for individual latitude-longitude boxes and the entire area. The $\Delta U$ $\left(U_{a}-U_{f}\right)$ and $\Delta V\left(V_{a}-V_{f}\right)$ for each box are also displayed in a grid. Some boxes contained no scatterometer values for a particular day and are marked N/A. For days 230,236 , and 248 , the respective $\Delta U$ values are $-1.29,1.12$, and $-1.03 \mathrm{~m} / \mathrm{s}$, while the $\Delta \mathrm{V}$ values are $0.57,1.34$, and $2.14 \mathrm{~m} / \mathrm{s}$. The average values of the boxed differences are somewhat lower, with $-0.63 \pm 2.04,0.36 \pm 1.19$, and $-0.91 \pm 1.93 \mathrm{~m} / \mathrm{s}$ for mean $\Delta \mathrm{U}$, and $0.05 \pm 2.09,0.58 \pm 2.13$, and $0.52 \pm 2.44 \mathrm{~m} / \mathrm{s}$ for mean $\Delta \mathrm{V}$, with all mean differences less than one standard deviation.

The difference between mean values for the entire region is thus larger than the difference expected within the smaller area of any latitude-longitude box. The increased difference is due in part to the greater spatial variability of the wind field when averaged across the larger area. The average component variances computed for the entire area, for both SASS and FNOC winds, are close in magnitude and day-to-day differences, with the only significant difference on day 248 , between the 
$U_{a}$ variance of $46.23 \mathrm{~m}^{2} / \mathrm{s}^{2}$ and the $U_{f}$ variance of $29.98 \mathrm{~m}^{2} / \mathrm{s}^{2}$. The boxed mean differences exhibit no obvious latitudinal, meridional, or temporal biases or trends, at least not evident over these three days. The boxed variances of the Atlas winds tend to be higher than the corresponding FNOC wind variances, for both $U$ (23 out of 30 ) and V (22 out of 30 ) components. The relatively greater variances of the Atlas winds is an expected result, since the higher resolution scatterometer winds should be more variable than those from the smoothed FNOC surface analysis. Those cases where the FNOC variances are higher generally correspond to coverage of strong circulation features, picked up completely by the FNOC analysis on its regular grid, but only partially covered by the satellite swath. This potential, but unresolvable, difference in spatial coverage within a given latitude-longitude box is one reason for regridding the satellite data to locations corresponding to those of the FNOC data. From this very limited set of data the most significant fact to emerge is the negative result that no readily apparent bias exists between the two fields. The limited methods of intercomparison (ie: boxed mean values) and associated poor resolution of the difference field dictate an alternative process, in which scatterometer and FNOC winds are colocated on a regular grid to facilitate statistical comparisons. 
Scatter plots of wind speed and direction

As a check on the mapped winds, the average daily standard deviations of the $\mathrm{U}$ and $\mathrm{V}$ components of the original and mapped scatterometer winds were plotted versus one another (Figure 15). Values fall along the $45^{\circ}$ line without much scatter, providing confirmation of the chosen mapping procedure. Scatter plots of Atlas vs FNOC wind speeds and directions over one-day periods are given in Figures 16 to 44. The speed plots simply show the degree of agreement about a $45^{\circ}$ line, while the direction plots have a slightly more difficult interpretation, since the periodic nature of compass directions means that values in the upper left and lower right corners also indicate closer agreement. The most obvious feature of nearly all the plots, especially those for direction, is the large degree of scatter present. The scatter tends to increase at higher wind speeds, in some cases about the $45^{\circ}$ line, and in others tailing off to one side. With the exception of days 229, 230, 231, 244, 247, and 248, the daily averaged Atlas wind speeds are greater than the FNOC wind speeds. No consistent trends are evident in the pattern of agreement as a function of wind speed. On some days (see days 225 and 235) the scatter seems evenly split between Atlas and FNOC, with or without offset from the $45^{\circ}$ line. On others (see days 232, 233, and 234) the pattern trails to the right at higher wind speeds, indicating that the higher Atlas winds were not picked up by the FNOC analysis. Day 248 shows an especially distinctive curve to the right for the highest Atlas wind speeds. During this period Hurricane Ella was moving along the east coast of North America, weakening and moving northeast over the open ocean by $12 \mathrm{Z}$ on day 248. The Atlas winds at this time, from revolution 1008 (Figure 45), are considerably higher than those from the corresponding FNOC analysis (Figure 46). The offset in time should not be a significant factor, since this satellite pass was only $\sim 40$ minutes prior to the analysis time. The difference between the two fields can be interpreted as either a too rapid weakening in the meteorological analysis, or an overestimation by the scatterometer, perhaps due to the extreme surface weather associated with the hurricane. Without additional information the exact cause cannot be determined with certainty, but it 
is a good example of possible differences in the two fields in response to a strong circulation feature.

There are also several days (see days 230, 246, and 247) in which high FNOC winds are not reflected by Atlas values, tilting the scattered points to the left. Day 244 shows this trend of greater FNOC speeds, at higher wind speed ranges, in addition to a very large scatter at the higher wind speeds. For day 230, revolution 744 in particular, the tilt seems to be due to the mapping, more than to a difference in the actual winds. The most northern box was extrapolated too far at its northern edge, and since the wind field magnitudes increase to the north, the extrapolated estimates are significantly lighter than the corresponding FNOC winds. The objective map of revolution 744 was one of the first made, and demonstrates the artificial differences that can be imposed by even slightly faulty mapping. More care was taken with subsequent maps to keep the boundaries of the estimate grids within the extent of the input data. For days 244 and 247, the likely cause of the higher FNOC winds is again related to the passage of Hurricane Ella. On day 244 the hurricane was intense, and small in area, and the rapid change in winds over short distances could easily account for the large scatter seen in the Atlas vs. FNOC scatter plot at the higher wind speeds. Strongest winds were present during this period in the fully developed hurricane, and limited comparisons to high-quality surface wind data have shown some tendency for the SASS to underestimate the highest $(\geq 20 \mathrm{~m} / \mathrm{s})$ wind speeds (Jones et al., 1982). This may be the case in this situation, especially since, as shown above, when the winds abated by day 248 the Atlas values became relatively larger than those of the FNOC analysis. For day 246 (with no satellite passes over Hurricane Ella), the probable cause of the relatively high FNOC winds is that the strongest winds in a developing tropical storm at the southern edge of the grid area were not picked up in the available Atlas data. The consistent differences exhibited by these specific cases were not reflected through the entire data set. Despite the large amount of scatter about any estimated regression line on a particular day and the different slopes of those lines for different days, the overall trend is towards agreement between Atlas and FNOC wind speeds across the entire data set. 
Agreement in wind directions also follows the same general trend, with even more variation and no easily discernible pattern. Differences of up to $180^{\circ}$ are found, especially on days $228,229,230,234,235$, and 251 . Two factors can strongly influence directional differences, both due to slight mismatches in the sampling times and areas of the two fields. The first is the inherent variation under light and variable wind conditions (under high pressure cells for example), in which small differences in sampling times and locations, and integrating intervals could easily show up as observed differences. The second factor involves offsets in the locations of well-developed circulation patterns for the two fields, especially cyclones, where the actual winds sharply change direction over relatively short distances. The potential for differences from offset patterns is particularly large for intense features such as hurricanes, where the change of wind direction is extremely rapid and the wind speeds are very large. The differences resulting from offset patterns are "real", since the winds seen by each field are not quite the same. For most of this data set, the meteorological features tend to match up well, so the first factor has a more significant role in the average directional differences. The exception is again related to the passage of Hurricane Ella, and offsets in its location in the two fields lead to extreme directional differences. The difference in circulation patterns is evident in the Atlas winds from revolution 1008 (Figure 45), where the inferred location of the low pressure center is to the northeast of that of the day $24812 Z$ FNOC analysis (Figure 46). The broader circulation patterns associated with less intense cyclones are generally much more similar between the two fields and exhibit less extreme differences than the hurricane case. Overall, the comparisons of the individual wind vectors shown in these scatter plots show that there are significant differences in the fields, particularly in the generally larger magnitude of the Atlas winds. The wide scatter prevents resolution of other trends, however, at least of any pattern that remains consistent throughout most of the data.

In addition to the daily plots of wind speed and direction comparisons, various averaged quantities were plotted in the same fashion. These averaged values are shown in Figures 47 to 58 , and include various one-day and three-day period plots of mean speed and speed and vector standard deviation, as well as the means and standard 
deviations for the $\mathrm{U}$ and $\mathrm{V}$ components, including a separation into latitude bins. The form of the mean speed and vector standard deviations are the same as given for the field statistics. From the daily mean speed plot (Figure 47) it is obvious that the Atlas winds are higher on average. The spatial variability, as expressed by the standard deviation of the mean speed, seems to be generally the same, given the small sample size and fair amount of scatter (Figure 48). The vector standard deviation shows that the Atlas wind directions are slightly more variable on average than the FNOC values (Figure 48). The three-day average values show a similar pattern, with all average Atlas speeds greater than FNOC speeds, a nearly even split in the standard deviations of the mean speed, and all but one of the Atlas vector standard deviations larger than the corresponding FNOC value (Figures 49 and 50). Scatter plots of the daily mean values of the $\mathrm{U}$ and $\mathrm{V}$ wind components (Figure 51) show a trend of agreement along the $45^{\circ}$ line for $\mathrm{U}$ and a wider dispersion with more highly negative Atlas than FNOC values for V. For both components, and most markedly for eastward velocity, most of the average FNOC values are larger than the corresponding Atlas velocities. This can be partially attributed to the greater variability of the Atlas winds, however, which tends to reduce the mean $U$ and $V$ values as negative and positive velocities cancel each other. Eastward average velocities are split rather evenly between positive and negative, while northward velocities are mostly positive. This corresponds with expected average flow patterns over this area at this time of year.

Plots of the standard deviations of the $U$ and $V$ components (Figure 52) show general agreement between Atlas and FNOC variability, with greater dispersion for the northward values. For both components, especially the northward, most Atlas values are greater than the FNOC values. The relatively greater variability of the Atlas winds is expected, since the FNOC fields are smoothed over a larger area than the Atlas fields. The plots of $\mathrm{U}$ and $\mathrm{V}$ component means and standard deviations, grouped into latitude bins (Figures 53 to 58 ) exhibit many of the same patterns as the combined daily values. The Atlas standard deviations are greater and the mean FNOC values are slightly higher. The eastward velocities are negative (southerly) in the $20-30^{\circ} \mathrm{N}$ bin, increase and become positive in the $30-40^{\circ} \mathrm{N}$ bin, and decrease 
slightly in magnitude and scatter about zero in the $40-50^{\circ} \mathrm{N}$ bin, averaging positive in each latitude bin. The standard deviations for both components are smallest in the $20-30^{\circ} \mathrm{N}$ bin and of relatively similar magnitude in the two northern bins.

Difference field statistics

Various statistics on the $U$ and $V$ components of the Atlas-FNOC vector difference field for individual areas within each revolution are listed in Table 16, including the mean, variance, weighted variance, standard deviation, mean squared difference, and weighted mean squared difference. The distributions of the $\Delta U$ and $\Delta V$ component means, and the speed and direction of the difference vectors (formed from individual $\Delta U$ and $\Delta V$ components) are shown graphically in Figure 59. Most values of $\Delta \mathrm{U}, \Delta \mathrm{V}$, and $|\overrightarrow{\mathrm{U}}|$ fall within a $\pm 5 \mathrm{~m} / \mathrm{s}$ range, with a much smaller, but significant number in the -10 to $-5 \mathrm{~m} / \mathrm{s}$ range for $\Delta \mathrm{V}$ and 5 to $10 \mathrm{~m} / \mathrm{s}$ range for $|\Delta \overrightarrow{\mathrm{U}}|$. The difference vector direction, $\theta_{\Delta \overrightarrow{\mathrm{U}}}$, is evenly distributed among the various direction bins, with slightly more in the $180^{\circ}-300^{\circ}$ range (southeasterly in the meteorological sense). So the difference vector does not appear to have any preferential direction over this data set. The revolution and box number of the Atlas wind field and the synoptic analysis time of the matching FNOC wind field are listed in each case. The mean squared difference (MSD) is simply the expected value of the square of the difference between either the U or V Atlas and FNOC components:

$$
\operatorname{MSD}=\frac{1}{N} \sum_{i=1}^{N}\left(U_{A i}-U_{F i}\right)^{2}
$$

The weighted mean squared difference (WMSD) is weighted by the square of the Atlas field:

$$
\mathrm{WMSD}=\frac{\sum_{\mathrm{i}=1}^{\mathrm{N}}\left(\mathrm{U}_{\mathrm{Ai}}-\mathrm{U}_{\mathrm{Fi}}\right)^{2}}{\sum_{\mathrm{i}=1}^{\mathrm{N}} \mathrm{U}_{\mathrm{Ai}}^{2}}
$$

As with the weighted variance, the normalized mean squared difference is more useful for intercomparisons of values from different areas, since it removes the influence of wind strength on the MSD magnitude. Areas of stronger winds will tend to have larger mean squared differences than those of lighter winds, simply because the individual 
magnitudes are larger, but a more accurate measure is to determine which of two cases is proportionally larger, relative to the respective wind strengths, rather than in the absolute sense. The relative sizes of the means and variances of the two components are evenly split, with $|U|>|V| 71$ out of 144 times $(49 \%)$ and $\sigma_{u}^{2}>\sigma_{v}^{2} 70$ out of 141 times $(50 \%)$, with three cases of equal variance.

One-day averaged statistics for the $U$ and $V$ components of the vector difference field are listed in Table 17. The mean daily $U$ and $V$ magnitudes are quite small and less than one standard deviation in nearly all cases, with the only exceptions coming in the $\mathrm{V}$ component means on days 237 and 243. As expected, the oneday average means are lower than the "snapshot" values from individual areas, and the corresponding variances are higher than those for individual areas. The large variances of the averaged difference field components, with most weighted variances greater than 0.9 , combined with the low mean values, indicates a lack of obvious bias between the two fields over one day periods. The values of the $U$ component range from -1.73 to $1.15 \mathrm{~m} / \mathrm{s}$ and those of the $\mathrm{V}$ component from -3.13 to $1.36 \mathrm{~m} / \mathrm{s}$, with the largest magnitudes occuring during days 242 to 245 , particularly in the V component. Though the $\mathrm{V}$ component has a slightly greater range than $\mathrm{U}$, neither component is consistently larger than the other, with $|\mathrm{U}|>|\mathrm{V}| 13$ out of 29 times (45\%). The relative sizes of the weighted variances are also split evenly between the two components, with $\sigma_{\mathrm{u}}^{2}>\sigma_{\mathrm{v}}^{2} 15$ out of 29 times (52\%). The mean $\mathrm{U}$ component is nearly evenly split between positive (westerly winds) and negative (easterly winds) directions, with $\mathrm{U}$ positive 13 out of 29 times (45\%), while the mean $\mathrm{V}$ component shows more bias in sign, with $\mathrm{V}$ positive (southerly winds) only 8 of 29 times (28\%). So, though there does not seem to be an east-west directional bias between the Atlas and FNOC winds, the Atlas winds tend to be more southerly (negative) than the FNOC winds. Table 18 presents $U$ and $V$ component statistics, grouped into $10^{\circ}$ latitude bins and averaged over one-day periods.

In addition to various statistical quantities calculated for the $\mathrm{U}$ and $\mathrm{V}$ components of the difference field, mean speed and vector statistics were also computed. To calculate these values, the component difference field $(\Delta U$ and $\Delta V$ ) was treated as a 
vector, with the speed, $|\Delta \vec{U}|$, calculated as $\left(\Delta U^{2}+\Delta V^{2}\right)^{\frac{1}{2}}$, and the speed and vector variances calculated as shown in the preceding section. This method of calculating the mean speed generally yields values considerably larger than the difference of the magnitude of the Atlas and FNOC vectors, especially in cases where the Atlas and FNOC components are of opposite sign. So these mean speed vaules reflect differences is direction as well as magnitude. Table 19 contains these values for individual areas and instantaneous times, Table 20 has the same for the entire area averaged over one-day periods, and Table 21 contains values grouped into $10^{\circ}$ latitude bins and averaged over one day periods. The largest mean speed values for individual areas, such as 952 2, 9881,1008 2, and 1074 2, are all associated with circulation around strong low pressure systems, with Hurricane Ella in the first three cases. The rapid change in direction of these winds, across relatively short distances, results in large differences if the location of the low pressure center is even slightly offset between the Atlas and FNOC fields. A good example of this is Atlas revolution 1008 (Figure 45), mentioned in the previous section, where the inferred location of the low is displaced to the northeast, relative to that of the FNOC day $24812 \mathrm{Z}$ analysis (Figure 46 ). The standard deviation of the mean speed is always less than the mean value, with the largest variance in the areas of highest mean speed, such as those discussed above. The very large vector variance values for all these same areas also establish the significant impact of the directional differences. The one-day averages do not exhibit the extreme values of the individual areas, with the average mean speed remaining the same, and the speed and vector variances increasing slightly on average. The standard deviation of the mean speed is less thant the mean speed in all but one case, day 248 , which is associated with the passage of Hurricane Ella. An interesting trend in the daily averages is that both the speed and vector variance increase towards the end of the study period (late August and early September). The progressive increase is most likely associated with the stronger weather patterns that begin to track further south at that time (reaching the northern edge of the study area), as well as with the influence of Hurricane Ella. The daily averages, grouped by latitude, exhibit similar trends, and the standard deviation of the mean speed is greater than the mean value 
in every case. The most noticeable trend in these is the distinct increase of the mean speed, and speed and vector variances, from south to north. The largest values fall within the $40^{\circ}-50^{\circ} \mathrm{N}$ bin, and this supports the association of greater Atlas-FNOC differences with the stronger weather patterns that are generally found there. 
Wind stress curl

A primary use for synoptic scale wind fields is as a driving force for ocean circulation models, since the wind stress is the single most important factor in determining that circulation. The input of wind energy into the ocean through the surface stress is reflected in the directly wind-driven Ekman flow, in the geostrophic flow due to divergence of the Ekman flow, and in the indirectly wind-driven motions due to thermohaline forcing. Considering just the frictional Ekman layer at the surface, vertical integration of the Ekman equations, in combination with mass conservation, yields a simple relation between the vertical component of the curl of the wind stress and the vertical velocity at the base of the Ekman layer:

$$
\mathrm{f} \rho \mathrm{w}_{\mathrm{e}}=\hat{\mathrm{k}} \mathrm{curl} \vec{\tau}
$$

where:

$$
\begin{aligned}
& \mathrm{f}=\text { Coriolis parameter }(1 / \mathrm{s}) \\
& \rho=\text { water density }\left(\mathrm{kg} / \mathrm{m}^{3}\right) \\
& \mathrm{w}_{\mathrm{e}}=\text { vertical velocity at base of the Ekman layer }(\mathrm{m} / \mathrm{s}) \\
& \hat{\mathrm{k}} \mathrm{cur} \vec{\tau} \vec{\tau}=\text { vertical component of the wind stress curl }\left(\mathrm{N} / \mathrm{m}^{3}\right)
\end{aligned}
$$

The vertical velocity at the base of the Ekman layer, driven by the wind stress curl, then acts as an upper boundary condition on the interior circulation. In the interior of the ocean, beneath the Ekman layer, we expect the linear vorticity balance to hold:

$$
\beta \mathrm{v}=\mathrm{f} \frac{\partial \mathrm{w}}{\partial \mathrm{z}}
$$

where:

$$
\begin{aligned}
& \beta=\text { variation of the Coriolis parameter with latitude }(1 / \mathrm{ms}) \\
& \mathrm{v}=\text { meridional velocity }(\mathrm{m} / \mathrm{s}) \\
& \frac{\partial \mathrm{w}}{\partial \mathrm{z}}=\text { vertical gradient of vertical velocity }
\end{aligned}
$$

In the general case, the wind stress curl acts as a driving force on this interior flow, through the dynamics of the Ekman layer, but a special case illustrates a more direct 
connection between the circulation and wind stress curl. The Sverdrup relation is a simple form of the relation between the curl of the wind stress and the mass transport, found by vertically integrating the linear vorticity equation, neglecting lateral friction, and assuming that vertical velocity vanishes at the bottom. The resulting equation is:

$$
\beta \mathrm{M}_{\mathrm{y}}=\hat{\mathrm{k}} \operatorname{curl} \vec{\tau}
$$

where:

$$
M_{y}=\text { vertically integrated meridional transport }
$$

As discussed by Stommel (1965), the Sverdrup relation actually expresses a balance between the divergence of the Ekman transport and the divergence of the geostrophic transport. A further discussion of this dynamical balance and the relationship between the wind stress curl, Ekman transport, and geostrophic transport is given by Stommel (1965). It must be noted that, in addition to the frictional wind stress-driven motions described by these relations, motions forced by thermohaline processes are also significant. These are not discussed further, except to note that the surface wind field plays an important indirect role in this forcing as well, through its impact on the surface fluxes and on mixing in the water column. Further discussion of some basic aspects of thermohaline processes can be found in Pond and Pickard (1983) and Veronis (1981).

These relationships are then used by modelers to impose the direct and indirect forcing of the wind stress as a boundary condition on the ocean circulation. Pond and Pickard (1983) provide a brief review of various aspects of this process, including the typical wind fields used, the impact of wind field resolution, and the wind stress forcing used in several models. The usual choice of wind stress information is a climatological average wind stress field, several of which are available (Bunker, 1976; Han and Lee, 1983; Hellerman and Rosenstein, 1983). The shortest time scale of these fields is usually a monthly average, which is useful for computing mean circulation patterns, but which has limited application at shorter time scales. Large-scale ocean currents and ocean properties such as the sea surface temperature and vertical 
temperature distribution display pronounced spatial and temporal variability. The variations of ocean motions and properties are directly related to the variability of the surface wind field, most of which is lost in the averaged climatological fields. The coarse spatial and temporal resolution associated with climatological fields prevents their use in examining the detailed structure and evolution of the ocean circulation in response to wind forcing. In addition to this resolution problem, even the accuracy of climatology is questionable, since it does not necessarily reflect local conditions at any particular time. The results of forcing from an averaged wind field may be considerably different from the overall effect of a temporally evolving wind field. The synoptic fields discussed in this paper are more appropriate than the climatological mean fields for forcing on a daily basis, as is needed to update numerical forecast models for example. Since knowledge of the wind stress curl is so crucial, it is important to examine the wind stress curl values for the Atlas and FNOC wind fields, in addition to the wind velocities.

For each field, the first step is to calculate wind stress from the wind velocity, using the wind stress and drag coefficient formulations of Large and Pond (1981).

$$
\vec{\tau}=\rho \mathrm{C}_{\mathrm{D}}|\overrightarrow{\mathrm{U}}| \overrightarrow{\mathrm{U}}
$$

where:

$$
\begin{aligned}
& \tau_{\mathrm{u}}=\rho \mathrm{C}_{\mathrm{D}}|\overrightarrow{\mathrm{U}}| \mathrm{u} \\
& \tau_{\mathrm{v}}=\rho \mathrm{C}_{\mathrm{D}}|\overrightarrow{\mathrm{U}}| \mathrm{v} \\
& \overrightarrow{\mathrm{U}}=\text { vector wind velocity } \\
& \mathrm{C}_{\mathrm{D}}=\text { drag coefficient based on total wind magnitude, }|\overrightarrow{\mathrm{U}}|
\end{aligned}
$$

The wind magnitude, $|\overrightarrow{\mathrm{U}}|$, acts as a scale factor for each of the stress components, reflecting the fact that each velocity component will have an effect on the surface roughness, and that the combined effect will appear in both stress components. For the same reason, the drag coefficient, $C_{D}$, as a measure of the surface roughness, is 
based on the magnitude of the wind, rather than the individual $U$ and $V$ components. Once the stress is found at each point, the curl is calculated as:

$$
\operatorname{curl} \vec{\tau}=\frac{\partial \tau_{\mathbf{y}}}{\partial \mathbf{x}}-\frac{\partial \tau_{\mathrm{x}}}{\partial \mathrm{y}}
$$

where the partial derivatives are estimated by a simple finite difference. Using a finite difference method may underestimate the wind stress curl, especially if there are large spatial separations in the data. The underestimation is more of a concern in curl calculations made from wind stress averaged over longer periods, which tend to be more widely spaced than the $1^{\circ}$ latitude by $1^{\circ}$ longitude grid used here. Though the amount of underestimation due to finite differencing is reduced for the smaller grid spacing, it will still be present to some degree, yielding slightly conservative wind stress curl values.

With this scheme, the wind stress curl was computed at the interior points of each individual grid area for the Atlas and FNOC winds. These were then averaged for each grid and also over one day periods. Values for the wind stress curl, averaged over individual areas for the Atlas winds, are listed in Table 22. Included are the mean, variance, weighted variance, standard deviation, and the number of data points. These statistics are calculated in the same manner used previously for the wind velocity components. The average curl $\tau_{A}$ magnitude of $1.2 \times 10^{-7} \mathrm{~N} / \mathrm{m}^{3}$ is in agreement with the $O\left(10^{-7}\right)$ magnitudes generally expected (Pond and Pickard, 1983). The wind stress curl is positive in 96 of 145 times (66\%), which is an expected result, since estimates made from climatological winds tend to show positive wind stress curl north of approximately $30^{\circ} \mathrm{N}$ in the North Atlantic (Leetmaa and Bunker, 1978). The mean values are generally close to the respective standard deviations, with the standard deviations being larger a slightly greater percentage of the time, $\sigma_{\mathrm{A}}>\left|\operatorname{curl} \tau_{\mathrm{A}}\right| 85$ of 145 times (59\%), with both the same three times.

Wind stress curl averages for individual areas of the FNOC winds are shown in Table 23, in the same format as for the Atlas values. The average curl $r_{\mathrm{F}}$ magnitude of $1.0 \times 10^{-7} \mathrm{~N} / \mathrm{m}^{3}$ is again of the expected order of magnitude, and it is positive 103 of 145 times (71\%). As with the Atlas values, the mean curl $\tau_{\mathrm{F}}$ values are generally close 
to their respective standard deviations, with $\sigma_{F}>\left|\operatorname{curl} \tau_{F}\right| 74$ of 145 times (51\%), and equal three times. For both the Atlas and FNOC winds, the relatively large standard deviations indicate that there is considerable variation of the wind stress curl, even within the gridded areas. Comparing the relative magnitudes of values from the two fields in each area, $\left|\operatorname{curl} \tau_{A}\right|>\left|\operatorname{curl} \tau_{F}\right| 91$ of 145 times $(63 \%)$, with both the same three times. Both in an average sense (from all areas) and for individual grids, the Atlas winds tend to have a slightly greater wind stress curl magnitude than the FNOC winds. In general, the respective area averages from each field agree in sign, with both having the same sign 122 of 145 times (84\%). The average difference (in magnitudes) between mean curl values of the two fields is $8.1 \times 10^{-8} \mathrm{~N} / \mathrm{m}^{3}$, which is of the same order as the means, but is also less than the standard deviations, so the difference is often swamped by the variation of the two fields.

The Atlas and FNOC wind stress curl fields were also compared by examining contour plots of the wind stress curl values within individual boxes. For both the Atlas and FNOC fields, results from the contour plots are consistent with the area statistics, also reflecting the increased magnitudes and increased spatial variability of the wind stress curl in regions of more intense circulation. More quiescent areas are associated with broad patterns and smaller curl $\tau$ magnitudes, such as revolution 6642 , while areas of stronger weather are associated with more variable patterns and larger $\operatorname{curl} \tau$ magnitudes, such as 6643 (Figure 60) or the extreme case of 7151 (Figure 61). There is considerable variation in the amount of agreement between the plots for each field, with no obvious, consistent pattern to the differences. There is generally better agreement in regions of broad flow, with little spatial variability (revolution 10592 - Figure 62), partially because the Atlas and FNOC wind fields usually correspond well in those cases. Frequently, the basic structure of the two curl $\tau$ fields is similar, especially in the latitudinal variation, but colocated individual values can be quite different, either because the range of values is different or there is a bias between the two fields.

Revolution 8301 (Figure 63) illustrates both the similarities and differences that can be present in the matching contour plots. In this case, the same general trends are 
reflected in each field, including similar magnitudes across most of the area, the same tendency for $\operatorname{curl} \tau$ values to group in meridional bands, and similar zonal variation of the sign of the values. Large negative values are present in the east, becoming more positive to the west, and then again more negative yet further west. However, distinct differences are also present along the western edge, in the more negative Atlas values and the greater meridional variation of the FNOC winds. Also, the meridional bands do not have quite the same orientation for each field, and the values of the two fields are slightly different, such as along $52^{\circ} \mathrm{W}$, where the postive Atlas values reach much farther south than the positive FNOC values. A similar case is revolution 10082 (Figure 64), where the two fields have similar patterns, but the FNOC values seem displaced to the southeast relative to the Atlas values, and the Atlas values are more extreme, especially near $45^{\circ} \mathrm{N} 48^{\circ} \mathrm{W}$ and in the northeast corner. Despite the overall correspondence between the Atlas and FNOC curl $\tau$ fields seen in many plots, there are also many areas (both with light and strong winds) where neither the patterns nor values of the two fields are in agreement, such as revolution 10081 (Figure 65). A consistent difference between the two fields is not apparent in the curl $\tau$ contour plots, so a general conclusion cannot be drawn.

The point values of wind stress curl were also averaged over one-day periods, and the results are listed in Table 24 for the Atlas winds and in Table 25 for the FNOC winds. The same statistics are computed as in the previous case. As for the area averages, the daily averages tend to be positive, with $\overline{\operatorname{curl} \tau_{\mathrm{A}}}$ and $\overline{\operatorname{curl} \tau_{\mathrm{F}}}$ each positive 19 of 29 times (66\%). For both the Atlas and FNOC data, the standard deviation is greater than the mean for every day. Averaging the magnitudes of all the daily values also shows that the standard deviation is greater than the mean, with $\overline{\left|\sigma_{\mathrm{A}}\right|}=$ $2.9 \times 10^{-7} \mathrm{~N} / \mathrm{m}^{3}$ compared to $\overline{\left|\operatorname{curl} \tau_{\mathrm{A}}\right|}=6.7 \times 10^{-8} \mathrm{~N} / \mathrm{m}^{3}$, and $\overline{\left|\sigma_{\mathrm{F}}\right|}=3.3 \times 10^{-7} \mathrm{~N} / \mathrm{m}^{3}$ compared to $\overline{\left|\operatorname{curl} \tau_{\mathrm{F}}\right|}=5.3 \times 10^{-8} \mathrm{~N} / \mathrm{m}^{3}$. This large variation is most likely due to the wide spatial separation that occurs over the course of one day as data from different revolutions are combined. The relative magnitudes of the daily average wind stress curl reflect the same trend present in the individual areas, with $\left|\overline{\operatorname{curl} \tau_{A}}\right|>\left|\overline{\operatorname{curl} \tau_{F}}\right| 19$ of 29 times $(66 \%)$. The average $\left|\overline{\operatorname{curl} \tau_{A}}\right|$ value is also slightly larger than the average 
$\overline{|\operatorname{cur}| \tau_{F}} \mid$ value so, as for the individual areas, the Atlas wind stress curl tends to be slightly greater. The daily averages from each agree in sign most of the time, with both having the same sign 25 of 29 times (86\%). The average of the difference between the daily means is $4.0 \times 10^{-8} \mathrm{~N} / \mathrm{m}^{3}$, close to the mean values, but well under the standard deviation of each. The differences between the Atlas and FNOC curl values are of significant size relative to the mean values of each and show considerable scatter, with a tendency for the Atlas values to be slightly higher than the FNOC values: However, the large variation brings into question the significance of the larger magnitudes of the Atlas wind stress curl values, both for the smaller areas and averaged over one-day periods. 


\section{Summary and Conclusions}

There is a demonstrated need for better wind field information over the open ocean in order to drive the increasingly complex ocean circulation models. Most modelers have relied on fairly simple, artificial wind fields or climatological mean fields to provide wind forcing, but the advent of more sophisticated models requires realistic wind fields to provide a more highly resolved forcing function. Conventional wind data generally lack the combination of resolution and coverage needed for study of the temporal and spatial variability of the global ocean. Climatological fields provide global coverage, but do not have sufficient spatial or temporal resolution, while ship and buoy reports from field investigations provide highly resolved wind data in both space and time, but only over limited areas and for short periods of time. The conventional fields of most potential use are the synoptic analyses that combine winds derived from surface pressure fields and from available ship and buoy reports. Such synoptic data is available on a regular, frequent basis over much of the global ocean, but is inherently smoothed on the larger, pressure field scales, losing a considerable amount of the spatial variability in the wind field. Additionally, in areas such as the Southern Ocean, with limited surface pressure and wind reports, even the smoothed analyses are questionable. The requirement for a more highly resolved, global specification of the surface wind field led to the development of scatterometer theory, as a means of inferring surface wind information from a remotely sensed measurement of surface roughness from reflected radiation. This initial development culminated in the 1978 deployment of an operational scatterometer, SASS, on the SEASAT oceanographic satellite. Initial evaluations determined that SASS met its stated objectives (Jones et al., 1982) and, despite some later qualifications, the three months of data returned from this mission provide a unique source of useful scatterometer wind information.

With the deployment of an operational scatterometer, the primary sources of more extensive wind information will be the conventional, pressure-derived wind fields and scatterometer-derived wind fields. Both wind fields have the potential to provide 
considerably more information than climatological mean fields, with greatly increased temporal and spatial resolution. There are drawbacks and limitations to each, particularly in the smoothing of conventional winds on pressure-field scales and in the irregular sampling of the scatterometer measurements. However, each is reasonably accurate and the question is to select one as forcing for ocean models, or to determine the differences, if any, between them. Though the choice of the "best" field in an absolute sense is severely hindered by the virtual absence of accurate, widespread "surface truth" information, it is as important to evaluate the differences between the two fields. An investigation of these differences has been the focus of this study, using SASS winds available from R. Atlas at the Goddard Space Flight Center (through JPL), and 6-hourly synoptic analysis winds from the Fleet Numerical Oceanographic Center (FNOC).

A one-month subset of the Atlas data, from 12 August to 9 September 1978, over the western North Atlantic, from $20^{\circ}-50^{\circ} \mathrm{N}$ and $40^{\circ}-80^{\circ} \mathrm{W}$, was chosen for this study. The comparison required a regridding of the irregularly spaced SASS winds to a regular latitude-longitude grid to match the FNOC vector locations. The regridding was accomplished by objective mapping, following the procedure of Bretherton, Davis, and Fandry (1976). Comparison of the original and mapped Atlas wind fields, graphically and statistically, ensured that the mapping accurately reproduced the wind field. Comparison of the Atlas and FNOC wind fields to synoptic weather maps demonstrated qualitative agreement with surface weather features and general circulation patterns. Differences between the two fields were examined quantitatively by various means, including calculations of field statistics and boxed mean values, scatter plots of speed, direction, and standard deviation, statistical descriptions of the Atlas-FNOC difference field, and calculations of the wind stress curl values for each field.

The Atlas and FNOC fields are similar, in a broad statistical sense, across the entire study period, with wide scatter of the individual points about a general trend of agreement. The broad correspondence of the two fields is evident in the similar distribution of $\mathrm{U}$ and $\mathrm{V}$ components, for all individual vectors (Figures 11 and 13) 
and for those averaged over grid areas (Figures 12 and 14). The distribution of individual vectors into direction bins also follows a similar pattern for each, though more pronounced in the case of the FNOC winds (Figures 11 and 13). The broad agreement and large variation of individual points between the two fields is portrayed clearly in the Atlas vs. FNOC scatter plots, for both wind speed and direction. The relatively low mean values and high variances of the one-day average $\Delta U$ and $\Delta \mathrm{V}$ components indicate agreement in an average sense, within the limitations of individual field variation. The basic similarity of the Atlas and FNOC fields is also indicated by comparisons of the wind stress curl fields. The values of the overall average curl $\tau$ are very close, the curl $\tau$ values from each field tend to agree in sign over various averaging periods, and the difference between values from each, averaged over grid areas as well as one-day periods, is less than the variation of the fields within these areas and times.

Despite this general correspondence, relatively small but consistent differences do exist, reflecting the greater smoothing of the FNOC winds on larger scales, and the slightly greater Atlas wind magnitudes. The smoothing of the FNOC field is evident in the variances of the $\mathrm{U}$ and $\mathrm{V}$ components, averaged over grid areas, as the the Atlas variances are more frequently larger than the FNOC values (Figures 12 and 14). The Atlas winds also exhibit a greater range of directions for more of the wind vectors (Figures 11 and 13), indicating greater variability in direction. Scatter plots of the vector standard deviations (Figure 48) show the greater variance of the Atlas winds over one-day and three-day periods, as do the plots of standard deviations of the U and $\mathrm{V}$ components over the same periods, as well as grouped by latitude (Figures 52 to 58). The tendency for the Atlas wind magnitudes to be larger shows up in the greater numbers of the $\mathrm{U}$ and $\mathrm{V}$ components (from individual vectors and grid area averages) at higher wind speed ranges for the Atlas winds (Figures 11 to 14). Scatter plots of all wind speeds from single days, and the scatter plots of all one-day and three-day mean wind speeds show that Atlas values are greater than FNOC values most of the time. The scatter tilts toward larger Atlas values in 23 of the 29 daily plots, 25 of 29 of the mean daily Atlas wind speeds are higher, and all of the three-day 
mean Atlas wind speeds are higher. The consistency of this trend is striking, but can be assigned only limited significance, since in many cases the difference is less than the variation of the individual fields over the particular averaging period. Difference field statistics show that significant differences exist between individual vectors, but that the average differences are small, especially relative to the variation across a given averaging period or area. The nearly even division of $\Delta U$ and $\Delta V$ into positive and negative values and the relatively even distribution of the difference vector in various direction bins (Figure 59) indicates that there is no preferential direction to the individual difference vectors. For one-day averages, $\overline{\Delta U}$ is still split relatively evenly into positive and negative values, but $\overline{\Delta V}$ is negative in most cases, indicating that the daily mean Atlas winds are more southerly than the FNOC winds.

The general similarity and relatively minor differences between the Atlas and FNOC fields summarized above characterize most of the data in this study, but some extreme differences are present in limited areas. Though significantly different individual vectors are scattered throughout the data, regions of large and extensive differences are associated in particular with strong circulation features such as hurricanes. The large differences are due in large part to offsets in the location of the low pressure centers, since even relatively small changes can cause large differences to appear. The influence of strong circulation patterns is obvious in the difference field statistics, where the largest values, for individual points as well as various averaged quantities, are found in the vicinity of intense weather, especially Hurricane Ella. The mean speed and vector variances of the difference field vectors increase towards the end of the study period and also from south to north on average. Both the later time (September) and northern regions $\left(40^{\circ}-50^{\circ} \mathrm{N}\right)$ are associated with relatively stronger weather patterns. In addition to these vector differences, the response at the highest wind speed range is also significantly different for the two fields, as the FNOC values are generally higher. The relatively higher FNOC winds may reflect underestimation by the SASS, but the small numbers of vectors involved prevent any general conclusion. 
For this one month period, the Atlas and FNOC winds are similar in their basic circulation patterns, in the statistical descriptions of each field, and in the average agreement of individual vectors. Most of the differences that do exist lie within the considerable scatter due to the variation of each field. However, the extreme differences associated with intense circulation features have the potential to greatly reduce the overall agreement if their occurrence is frequent enough. The ultimate impact on ocean model forcing will depend on the particular area studied and the averaging period. In regions of frequent strong weather, the scatterometer and conventional winds could yield quite different results, especially at shorter time and space scales. Differences at the shorter scales would apply particularly to prediction models for forecasting, that depend strongly on the temporal and spatial variations of the input data. Assuming that the possible underestimation of the highest wind speeds by the SASS is resolved in subsequent scatterometers, and that the circulation patterns and inferred positioning of weather systems are reproduced more accurately by the scatterometer winds (Duffy and Atlas, 1986), the scatterometer information will be a significant improvement in these applications. For more quiescent regions and for data averaged over longer periods the differences in the two fields should be relatively minor. The slightly greater spatial resolution of the scatterometer winds will be mostly lost in averages over longer periods of time, and should give results similar to those of conventional field in those cases. 


\section{Appendix 1: Aspects of atmospheric boundary layer theory}

Motions in the turbulent, atmospheric boundary layer are described by MoninObukhov similarity theory, which quantifies the balance between turbulence produced by wind shear and by buoyancy. A different velocity scale is important in this theory:

$$
\mathrm{u}_{*}=(\tau / \rho)^{\frac{1}{2}}=|\overline{\mathrm{uw}}|^{\frac{1}{2}}
$$

or,

$$
\tau=\rho\left(\mathrm{u}_{*}\right)^{2}
$$

where:

$$
\mathrm{u}_{*}=\text { friction velocity }(\mathrm{m} / \mathrm{s})
$$

The friction velocity, $\mathrm{u}_{*}$, is a surface flux parameter used as another means of representing wind stress, in this case the kinematic stress. Also introduced is the MoninObukhov scaling length, L, which gives the height at which the turbulent energy production from non-neutral buoyancy and from mechanical shear in the neutral case are equivalent (Large and Pond, 1981). Essentially, it is the height at which shear and buoyancy effects are approximately the same. $\mathrm{L}$ can be expressed as:

$$
\mathrm{L}=-\frac{\mathrm{u}_{*}^{3} \mathrm{~T}_{\mathrm{a}} \rho \mathrm{c}_{\mathrm{p}}}{\kappa \mathrm{gH}} \approx \frac{\left(\mathrm{C}_{\mathrm{D}}\right)^{\frac{1}{2}} \mathrm{~T}_{\mathrm{a}} \mathrm{U}_{10}^{2}}{\kappa \mathrm{g}\left(\mathrm{T}_{\mathrm{s}}-\mathrm{T}_{\mathrm{a}}\right)}
$$

where:

$$
\begin{aligned}
& T_{a}=\text { air temperature at surface }(\mathrm{K}) \\
& \mathrm{c}_{\mathrm{p}}=\text { specific heat capacity of air }(1030 \mathrm{~J} / \mathrm{kgK}) \\
& \kappa=\text { von Karman's constant }(\sim .35) \\
& \mathrm{g}=\text { acceleration of gravity }\left(9.8 \mathrm{~m} / \mathrm{s}^{2}\right) \\
& \mathrm{H}=\text { flux of sensible heat }\left(\mathrm{W} / \mathrm{m}^{2}\right) \\
& \mathrm{U}_{10}=\text { mean wind speed at } 10 \mathrm{~m} \text { above mean sea level }(\mathrm{m} / \mathrm{s}) \\
& \mathrm{T}_{\mathrm{s}}=\text { mean sea surface temperature }(\mathrm{K})
\end{aligned}
$$$$
\text { (Stewart, 1985) }
$$ 
In both of these forms it is assumed that temperature effects are much greater than those of humidity, which is generally true for mid-latitudes, but does not generally hold in the tropics or under conditions of high evaporation, requiring adjustment to the equations in those cases (Stewart, 1985). A more general form can be found in Pond et al. (1971). For typical values of air-sea temperature difference, $L$ is usually greater than 30 meters, with the possible exception of very light wind conditions. Since this height is above the area most crucial to scatterometry, stability plays a secondary role and enters as adjustments to profiles derived by assuming a neutrally stable atmosphere.

Neglecting stability, the velocity profile is logarithmic with height. The stability corrections are functions of the non-dimensional stability parameter, $\frac{Z}{L}$, where $z$ is the height above the surface and:

$$
\begin{array}{ll}
\frac{\mathrm{Z}}{\mathrm{L}}=0 & \text { neutral stability } \\
\frac{\mathrm{Z}}{\mathrm{L}}>0 & \text { stable conditions } \\
\frac{\mathrm{Z}}{\mathrm{L}}<0 & \text { unstable conditions }
\end{array}
$$

This yields the mean wind profile, in gradient form:

$$
\phi_{\mathrm{m}}=\frac{\kappa \mathrm{z}}{\mathrm{u}_{*}} \frac{\mathrm{du}}{\mathrm{dz}}
$$

where:

$$
\begin{aligned}
& \mathbf{z}=\text { height above mean sea level }(\mathrm{m}) \\
& \phi_{\mathbf{m}}=\phi_{\mathbf{m}}\left(\frac{\mathrm{Z}}{\mathrm{L}}\right)=\text { dimensionless wind shear }
\end{aligned}
$$

(Businger et al., 1971)

Integrating this yields the actual profile:

$$
\mathrm{U}_{\mathrm{z}}=\frac{\mathrm{u}_{*}}{\kappa}\left(\ln \left(\frac{\mathrm{z}}{\mathrm{z}_{\mathrm{o}}}\right)+\phi_{\mathrm{m}}\left(\frac{\mathrm{z}}{\mathrm{z}_{\mathrm{o}}}\right)\right)
$$

where:

$$
\begin{aligned}
& \mathrm{U}_{\mathrm{z}}=\text { wind speed at some height } \mathrm{z}(\mathrm{m} / \mathrm{s}) \\
& \mathrm{z}_{\mathrm{o}}=\text { roughness parameter }(\mathrm{m})
\end{aligned}
$$


Since $\phi_{\mathrm{m}}(0)=1$, the neutral wind profile is logarithmic with height, as required. The form of $\phi_{m}$ is known only empirically, with one formulation as:

$$
\begin{array}{ll}
\phi_{\mathrm{m}}=\left(1-15 \frac{\mathrm{Z}}{\mathrm{L}}\right)^{-\frac{1}{4}} & \left(\frac{\mathrm{Z}}{\mathrm{L}}<0\right) \\
\phi_{\mathrm{m}}=\left(1+4.7 \frac{\mathrm{Z}}{\mathrm{L}}\right) & \left(\frac{\mathrm{Z}}{\mathrm{L}}>0\right) \\
\phi_{\mathrm{m}}=1 & \left(\frac{\mathrm{Z}}{\mathrm{L}}=0\right)
\end{array}
$$

(Businger et al., 1971)

Stewart (1985) shows that if the influence of stability is relatively weak, $\phi_{\mathrm{m}}$ can be expanded in a power series, yielding the profile solution:

$$
\mathrm{U}_{\mathrm{z}}=\frac{\mathrm{u}_{*}}{\kappa}\left(\ln \left(\frac{\mathrm{z}}{\mathrm{z}_{\mathrm{o}}}\right)+\beta\left(\frac{\mathrm{z}-\mathrm{z}_{\mathrm{o}}}{\mathrm{L}}\right)\right)
$$

where:

$$
\begin{array}{ll}
\beta=4.7 & \frac{\mathrm{Z}}{\mathrm{L}}>0 \\
\beta=3.8 & \frac{\mathrm{Z}}{\mathrm{L}}<0
\end{array}
$$

The major effect of stability is simply to shift the velocity profile away from the logarithmic form at non-neutral conditions. To estimate stress with a drag law from this profile, either the velocity or drag coefficient must be corrected for stability.

Given a similar equation for the virtual temperature profile and the expression for $\mathrm{L}$, we have a closed set of equations that can be solved for the fluxes given the profiles, or vice versa. However, the solution depends on finding a suitable expression for the roughness parameter, $z_{o}$ (Halberstam, 1980). This parameter arises from the mathematical process and can be considered a statistical description of the density and height of the roughness elements ( $W u, 1980)$. In the equations, $z_{0}$ represents the height, close to the surface, at which $U$ vanishes. In reality the velocity does not vanish, because, in the few centimeters immediately above the surface, viscosity effects become important, and the above relations are no longer valid. For solid surfaces $z_{o}$ is closely related to surface roughness and usually taken as constant, but a constant $z_{0}$ is not applicable to the moving ocean surface. Since wave heights (which form 
the roughness elements) are changed by the wind, $z_{0}$ has a pronounced wind speed dependence. The exact form of this dependence is not known, and many studies have been done to specify an empirical relation between $z_{0}$ and $u_{*}$. Charnock (1955) assumes that the shorter, steeper waves are primarily responsible for variations in the relevant roughness parameter and that these are usually in equilibrium with the local wind. His predicted form for $\mathbf{z}_{\mathrm{o}}$ was:

$$
\mathrm{z}_{\mathrm{o}}=\frac{\mathrm{au}_{*}^{2}}{\mathrm{~g}}
$$

where:

$$
a=a \text { proportionality constant }(0.0156)
$$

This information fits the observations well and also fixes the form of the drag coefficient, since $C_{D}$ is related to $u_{*}$. The relation is rationalized physically by Wu (1968), as an equation of state that characterizes the equilibrium between wind and waves with gravity waves acting as roughness elements (Wu, 1980). Further refinements to this form have been made, both those that assume a unique relation (Cardone, 1969; Garratt, 1977) and those that also incorporate further adjustments for swell and wind fetch (Kitaigorodskii and Zaslavskii, 1974). Garratt (1977) reviews several of these and concludes that a simple $z_{0}-$ to- $u_{*}$ relation is adequate, of the form:

$$
z_{o}=1.469 \times 10^{-5} u_{*}^{2}
$$

(CGS units)

Halberstam (1980) showed that the $u *$ derived from different forms of $z_{0}$ did not correlate significantly differently with backscatter measurements. The absence of significant differences is mostly a function of the considerable scatter in the SASS backscatter data, since it tends to swamp the differences due to different $z_{o}$ formulations.

As mentioned above, this parameterization of $z_{0}$ also fixes the form of the drag coefficient, since $C_{D}=\left(\frac{u_{*}}{U_{10}}\right)^{2}$, then using the derived form of $z_{o}$ in the log profile:

$$
C_{D}=\left[\frac{\kappa}{\ln \left(\frac{g^{z}}{\mathrm{au}_{*}^{2}}\right)}\right]^{2}
$$


With known values of $\kappa, \mathbf{g}, \mathbf{z}$, and $\mathbf{a}$, these equations can be solved numerically to yield $C_{D}\left(U_{10}\right)$ (Stewart, 1985). Many measurements have been made to determine an empirical $\mathrm{C}_{\mathrm{D}}$-to- $\mathrm{U}_{\mathrm{ref}}$ relation, both through direct and indirect techniques (ie: Smith, 1980; Large and Pond, 1981). The specification of wind speed dependence has been a major issue. Measurements made by Smith (1980), Large and Pond (1981), and others show a definite, though widely scattered, increase of the drag coefficient with wind speed. Wu (1980) uses scaling laws for $C_{D}$ to explicitly demonstrate that $C_{D}$ must increase with wind speed if both the wind speed follows a logarithmic profile and the Charnock relation holds. Physically, this increase, especially at higher wind speeds, can be tied to the different character of the ocean surface, as compared to a solid surface. Over a solid surface, as wind speed increases the viscous sublayer becomes thinner and roughness elements begin to protrude through it, increasing the roughness length (and drag coefficient). In the fully rough condition, the elements are completely exposed and since the roughness length will not increase with further increase of velocity, the drag coefficient is constant. On the other hand, on the ocean surface the short waves are the roughness elements and these continue to grow with increasing wind speed, even though a fully rough condition exists. So the drag coefficient continues to increase with increasing wind velocity. One notable aspect of ocean surface roughness is the presence of two markedly different regimes for lower and higher wind speeds. Within the atmospheric boundary layer, under neutral or unstable stability conditions, instabilities and large-scale coherent structures develop for winds higher than about $7 \mathrm{~m} / \mathrm{s}$ (Brown, 1986). This change of behavior in the boundary layer flow is reflected by a discontinuity in plots of surface roughness versus backscatter. This pattern has been recognized for quite some time - see Woiceshyn et al. (1986) for a more complete discussion.

The discontinuous behavior of $C_{D}$ cannot be fit with a single linear relation, in other words, by choosing a constant drag coefficient over the entire range of wind speeds. The alternative generally chosen is to fit two linear relations, across lower and higher wind speed regimes. Large and Pond (1981) have shown that a form of the drag coefficient that is constant at low wind speeds and varies linearly with $U$ at 
higher wind speeds fits the observations best. They relate the neutral drag coefficient referenced to $10 \mathrm{~m}\left(\mathrm{C}_{\mathrm{DN} 10}\right)$ and the wind speed at $10 \mathrm{~m}\left(\mathrm{U}_{10}\right)$ :

$$
\mathrm{C}_{\mathrm{DN} 10}= \begin{cases}1.2 \times 10^{-3} & \mathrm{U}_{10} \leq 11 \mathrm{~m} / \mathrm{s} \\ \left(0.49+.065 \mathrm{U}_{10}\right) \times 10^{-3} & \mathrm{U}_{10}>11 \mathrm{~m} / \mathrm{s}\end{cases}
$$

They also provide a method for calculating stress from wind speed at other heights, using this formula and the air-surface temperature difference. The variability of the drag coefficient with wind speed affects transient forcing as well as the mean wind stress. Variable drag coefficients have been shown to yield transient forcing that is approximately $30 \%$ higher than that for constant coefficients (Smith, 1980). The increased contribution of variable drag coefficients is an important consideration in selecting the forcing parameterizations and U-to- $\tau$ conversion, if necessary, for ocean modeling. With these relations, wind speed at various levels in the surface layer can be related to wind speed near the surface, and to stress on the surface through a drag coefficient. Stewart (1985) outlines the procedures: 1) Given the wind velocity at some height, $U_{z}$, and the air-sea temperature difference, find $L$ and estimate $\left.\frac{Z}{L} ; 2\right)$ If $\frac{Z}{L}$ is negligible and stability effects can therefore be ignored, use the logarithmic wind profile and Charnock's value of $z_{0}$ to find $U_{\text {ref }}$ (usually at $10 \mathrm{~m}$ ); 3) If $\frac{Z}{L}$ is small, but not negligible, correct $U_{z}$ to the value it would have if $\frac{Z}{L}=0$ (at the same height), then find $U_{r e f}$ with the $\log$ profile; 4) If $\frac{Z}{L}$ is not small, then $U_{r e f}$ can be estimated by numerical integration of the velocity profile, but it is generally better to get a new measurement of $U_{z}$ at a lower height. The extrapolations made for large $\frac{Z}{L}$ values are not very accurate. 


\section{Appendix 2: Ocean waves and energy transfer}

Capillary waves have wavelengths of about two centimeters, at which scale surface tension is a dominant influence. The sea surface can be described with spectra calculated from a three-dimensional Fourier transform, and the spectrum of windgenerated gravity waves is well known (Stewart, 1985). Spectra for the higher frequency capillary waves are not as well understood, nor is the actual spectra of the entire sea surface. Lleonart and Blackman (1980) discuss spectral characteristics of capillary waves and summarize some high-frequency wave measurements. Their studies indicate that the shape of the frequency spectrum, over the capillary wave range, depends on friction velocity, viscosity, and surface tension, and therefore on the local wind field. The height of small wavelength waves depends on balancing the input of energy from the wind and from other waves through wave-wave interactions with the loss of energy through viscosity, surface films, and other wave-wave interactions (Stewart, 1985). The method by which wind energy is input to capillary waves is still not completely understood, as discussed by Phillips (1977). Variations in both surface pressure, a normal force, and shear stress, a tangential force, contribute to wave growth: Phillips (1977) makes a distinction between two types of stress fluctuations, those generated by atmospheric turbulence and those induced by flow over the wavy surface. Turbulent stresses, due to their random nature, tend to contribute energy across a large frequency range, while the induced stresses contribute energy more selectively, enhancing the growth of only particular wave components (Phillips, 1977). An important issue is the allocation of the total momentum transfer, between the fraction associated with tangential stress, which is assumed to correlate with capillary waves, and the portion associated with the normal force of form drag, acting on the longer wave components (Stewart, 1985). Kitaigorodskii (1970) summarizes some of this work and finds that $70-85 \%$ of the momentum transfer to waves is linked to capillary waves, and the rest to longer waves. Theoretical calculations (Brooke Benjamin, 1959; Miles, 1962) and experimental observations (Kendall, 1970) indicate that 
the normal stresses are more important than the tangential stresses in energy transfer. Pierson et al. (1986) cite this work in their choice of a new physical parameter to relate to microwave backscatter, discussed in Section 2. The presence of significant energy transfer to the longer waves means that this long wave energy input must relate nearly constantly to the short wave energy input in order for the assumed linear wind-to-short wave spectra relations to hold (Brown, 1986). While an approximately constant relation holds for the near steady state conditions commonly found over the ocean, it does not hold under non-steady state conditions, such as those found in the vicinity of fronts and other weather systems. Phillips (1977) formulates an expression for the air-ocean energy flux that portion energy into wave motion and currents. It includes terms for the flux of energy into waves alone, for energy flux from normal and tangential stress variations, for the loss of mean flow energy from Reynold's stresses of the induced motion working against the mean velocity gradient, and for energy loss from wave-induced motion from molecular viscosity or variations in the turbulent Reynold's stress (Phillips, 1977).

In addition to the role of long waves in the partition of transferred energy, the presence of these waves may attenuate or enhance short wave growth, depending on the relative magnitude and orientation of the two (Phillips, 1978). The short wave shape, and therefore the associated surface roughness, can also change due to wave-current interactions, under the same wind conditions (Longuet-Higgins, 1978). Rain can significantly alter the short wave spectra, primarily by causing damping at higher rates of fall. Sea surface temperature variations affect short waves through the associated changes in viscosity, up to a factor of two across a $0-30^{\circ} \mathrm{C}$ temperature range (Pierson et al., 1986). Mixed layer dynamics, such as those responsible for Langmuir circulations can also significantly affect short wave spectra (Brown, 1986). These effects illustrate the complex system of multiple, interacting processes that complicate the basic picture of energy flux to the waves as a tangential stress correlated with short wave elevation and a normal stress in phase with the slopes of the longer waves. 


\section{Appendix 3: Aspects of radar scatter theory}

Microwave scatterometry is based to a large extent on known principles of radar scatter from rough surfaces. Calculating the backscatter from incident radiation on rough surfaces requires matching the electric and magnetic fields of the radiation across a boundary of known shape. Specifically, the scattering cross-section, $\sigma$, can be written as $4 \pi$ times the ratio of the scattered radiant intensity at the receiver to the power density incident on the area:

$$
\sigma=4 \pi \mathrm{A} \cos \theta\left(\frac{\mathrm{I}_{\mathrm{B}}}{\Theta_{\mathrm{i}}}\right)
$$

where:

$$
\begin{aligned}
& \sigma=\text { backscatter cross-section }\left(\mathrm{m}^{2}\right) \\
& \mathrm{A}=\text { surface area }\left(\mathrm{m}^{2}\right) \\
& \theta=\text { incidence angle measured from vertical } \\
& \mathrm{I}_{\mathrm{B}}=\text { radiant intensity }(\mathrm{W} / \mathrm{sr}) \\
& \Theta_{\mathrm{i}}=\operatorname{radiant} \text { flux }(\mathrm{W})
\end{aligned}
$$

(Stewart, 1985)

The incidence angle, $\theta$, is the angle between the incident radar beam and the local surface vertical. Power density is the radiant flux, $\Theta_{j}$, divided by the projected area, $A \cos (\theta)$. Using the radar equation, an expression for the received power can be found in terms of the transmitted power and the scattering cross section. Stewart (1985) develops an expression of this type for a point target. Since the sea is a distributed, rather than a point target, the chosen radiation measure is the normalized radar backscatter cross section (NRCS), $\sigma^{\circ}$. The dimensionless $\sigma^{\circ}$ is a ratio of the reflected to incident energy across a unit surface area, rather than the projected area. From these relations we can, in theory, find the power scattered toward the radar receiver if the form of $\sigma^{o}$ is known. So the problem is to find $\sigma^{\circ}$ for a given sea surface.

Current approaches to wave scattering from statistically rough surfaces fall into two categories: 1) specular or tangent-plane theory for slightly wavy surfaces, and 
2) Bragg-resonance theory for slightly rough surfaces. These two physical mechanisms take place over different ranges of incidence angles, with specular scatter at near vertical $\theta$, and Bragg scatter at larger angles. Specular scatter is a mirror-like reflection from wave facets that are oriented to reflect energy directly back toward the radar receiver. Because the direct reflection comes from wave slopes perpendicular to the incidence angles, and the fact that ocean wave slopes are rarely more than $20^{\circ}$, this mechanism is restricted to a small range of incidence angles. To apply this mechanism, wave facet shape must be specified, an estimate made of the probability of proper facet orientation made, and then the energy reflected by the facet calculated (Stewart, 1985). The estimate is generally made by use of a tangent plane approximation, through either a physical optics or geometrical optics approach. Stewart (1985) discusses the process in more detail, with expressions that sum the scatter over the incidence area, weighted by a probability distribution of surface slopes. The sea surface slopes are assumed to have a Gaussian distribution that is anisotropic about the wind direction. Those ocean wave lengths that are shorter than the radar wavelength do not contribute to this type of scatter. The return from specular scatter is isotropic in azimuth, which means that only magnitude and not direction information can be determined.

At larger incidence angles, the number of facets available for direct scatter decreases rapidly and another mechanism dominates the scatter. This is Bragg scatter, a resonant reflection from those wave components that match the projected radar wavelength on the ocean surface. It can be described as the combination of scattering from different surface elements, which, through constructive interference enhances the scattering from regular surface structures with favorable wavelengths, and through destructive interference diminishes all other reflections. The physical principle behind this type of scatter is that of a diffraction grating, and the radiation scatters in directions set by spacing and orientation of the regular surface elements relative to the radar wavelength (Stewart, 1985). The ocean surface can be viewed as a superposition of plane waves, and considering scattering as a linear process, then each individual component acts as a diffraction grating for a particular radiation wavelength. Since 
this resonant scatter adds coherently in the far field, with power given by Bragg's equation, it is known as Bragg scatter. Resonant scatter theory can be used with a physical optics approach to relate the backscatter measurement to the ocean surface. In a formulation developed by Wright (1968), using linearized boundary conditions, the backscatter cross section is directly proportional to the mean spectral density of the short-wavelength ocean waves:

$$
\sigma^{o}(\theta, \mathrm{k})_{\mathrm{ij}}=16 \pi \mathrm{k}_{\mathrm{r}}^{4} \cos ^{4} \theta\left|\mathrm{g}_{\mathrm{ij}}\left(\theta, \varepsilon_{\mathrm{r}}\right)\right|^{2} \Psi\left(\mathrm{k}_{\mathrm{x}}, \mathrm{k}_{\mathrm{y}}\right)
$$

where:

$$
\Psi\left(k_{x}, k_{y}\right)=2 \text {-D Fourier spectrum of surface height fluctua- }
$$

tions

$$
\begin{aligned}
& \mathrm{g}_{\mathrm{ij}}\left(\theta, \varepsilon_{\mathrm{r}}\right)=\text { first-order scattering coefficients } \\
& \varepsilon_{\mathrm{r}}=\text { complex dielectric constant of seawater } \\
& \mathrm{k}_{\mathrm{r}}=\text { radar wavenumber } \\
& \theta=\text { incidence angle }
\end{aligned}
$$

The actual dependence is on the spectrum of ocean wave slopes, but since that is linearly related to the surface height spectrum (ie: to the wave height spectrum), one can be used in place of the other (Stewart, 1985). This form combines the spectral densities of waves traveling toward and away from the antenna, since their respective doppler shifts are indistinguishable from each other.

For resonant scatter theory to hold, one condition is that $k_{r} h \ll 1$, where $h$ is the wave height. This condition is not satisfied for the short radar wavelengths used with capillary waves, but the scatter from these waves is handled by considering the ocean as a composite surface (Stewart, 1985). Composite surface theory assumes that the ocean surface has two separable scales of motion, with short-wavelength ripples superimposed on the longer gravity waves (Figure 66). Wright (1968) and Bass et al. (1968) first developed the basic principles of composite surface scattering theory, characterizing backscatter from the actual sea surface over the Bragg scatter range of incidence angles. Over small areas the Bragg scatter is calculated from the local wave 
field and local surface orientation. A probability distribution based on the slopes of the longer waves can then be used to integrate the local scatter values over a larger area. These mechanisms valid at intermediate incidence angles are then combined with the specular scatter mechanism at small incidence angles to produce a backscatter model for the ocean over a large range of incidence angles (Valenzuela, 1978). Laboratory and field experiments (see Stewart, 1985 for partial summary) support the two-scale Bragg scattering model over incidence angle ranges of approximately $20^{\circ}<\theta<70^{\circ}$ for vertical polarization and $20^{\circ}<\theta<60^{\circ}$ for horizontal polarization. For $\theta<20^{\circ}$, specular scatter starts to interfere, and it becomes the dominant scattering process for incidence angles on the order of the rms wave slope, roughly $5^{\circ}-8^{\circ}$ for wind generated waves. In the $10^{\circ}-20^{\circ}$ incidence angle range, the backscatter is relatively insensitive to wind speed. At the largest incidence angles $\left(\theta>60^{\circ}\right)$, wedge diffraction may become an important mechanism (Lyzenga et al., 1983), and shadowing by larger waves may prevent signal reception. Also, at these large angles, the intermittent signal scattered from white caps may be as strong as that from Bragg scatter, significantly contaminating the return. 


\section{REFERENCES}

Atlas, D., R.C. Beal, R.A. Brown, P. De Mey, R.K. Moore, C.G. Rapley, and C.T. Swift, 1986, Problems and future directions in remote sensing of the oceans and troposphere: A workshop report. J. Geophys. Res., 91, 2525-2548.

Atlas, R., A.J. Busalacchi, M. Ghil, S. Bloom, and E. Kalnay, 1987, Global surface wind and flux fields from model assimilation of Seasat data, J. Geophys. Res., 92, $6477-6487$.

Bass, F.G., I.M. Fuks, A.I. Kalmykov, I.E. Ostrovsky, and A.D. Rosenberg, 1968, Very high frequency radiowave scattering by a disturbed sea surface, 2, Scattering from an actual sea surface. IEEE Trans. Antennas Propag., AP-16, 560-568.

Baker, W.E., R. Atlas, E. Kalnay, M. Halem, P.M. Woiceshyn, S. Peteherych, and D. Edelmann, 1984, Large-scale analysis and forecast experiments with wind data from the Seasat A Scatterometer, J. Geophys. Res., 89, 4927-4936.

Boggs, D.H., 1981, The Seasat scatterometer model function: The genesis of SASS 1, JPL Int. Doc. 622-230, Jet Propul. Lab., Pasadena, CA.

Boggs, D.H., 1982, Geophysical Data Record User's Handbook: Scatterometer. Jet Propul. Lab. Rept. No. JPL D-129, Pasadena, CA.

Brooke Benjamin, T., 1959, On the generation of surface waves by shear flows, $J$. Fluid Mech., 13, 433-448.

Brown, R.A., 1983, On a satellite scatterometer as an anemometer, J. Geophys. Res., 88, 1663-1673.

Brown, R.A., 1986, On satellite scatterometer capabilities in air-sea interaction, $J$. Geophys. Res., 91, 2221-2232.

Bunker, A.F., 1976, Computations of surface energy flux and annual air-sea interaction cycles of the North Atlantic ocean, Mon. Weather Rev., 104, 1122-1140.

Businger, J.A., J.C. Wyngaard, Y. Izumi, and E.F. Bradley, 1971, Flux-profile relationships in the atmospheric surface layer, J. Atmos. Sci., 28, 181-189.

Cardone, V.J., 1969, Specifications of the wind distribution in the marine boundary layer for wave forecasting, New York University Rep. TR69-1,67pp.

Charnock, H., 1955, Wind stress on a water surface, Quart. J. Roy. Meteorol. Soc., 81, 639-640.

Davidson, K.L., G.E. Schacher, C.W. Fairall, and J.D. Jarrell, 1981, Observational results pertaining to scatterometer interpretation, In Oceanography from Space, J.F.R. Gower, ed., Plenum Press, New York, 597-606.

Duffy, D.G., and R. Atlas, 1986, The impact of Seasat-A Scatterometer data on the numerical prediction of the Queen Elizabeth II storm, J. Geophys. Res., 91, 22412248. 
Fischer, R.E., 1972, Standard deviation of scatterometer measurements from space, IEEE Trans. Geosci. Electron., GE-10, 106-113.

Freilich, M.H., and D.B. Chelton, 1986, Wavenumber spectra of Pacific winds measured by the Seasat scatterometer, J. Phys. Oceanogr., 16, 741-757.

Garratt, J.R., 1977. Review of drag coefficients over oceans and continents, Mon. Weather Rev., 195, 915-929.

Gohil, B.S., and P.C. Pandey, 1985, An algorithm for retrieval of oceanic wind vectors from the simulated SASS normalized radar cross-section measurements, $J$. Geophys. Res., 90, 7307-7311.

Han, Y.J., and S.W. Lee, 1983, An analysis of monthly wind stress over the global ocean, Mon. Weather Rev., 111, 1554-1566.

Halberstam, I., 1980, Some considerations in the evaluation of Seasat-A Scatterometer (SASS) measurements, J. Phys. Oceanogr., 10, 623-632.

Hellerman, S., and M. Rosenstein, 1983, Normal monthly wind stress over the world ocean and error estimates, J. Phys. Oceanogr., 13, 1103-1104.

Hofman, R.N., 1982, SASS wind ambiguity removal by direct minimization, 1, Mon. Weather Rev., 110, 434-445.

Hofman, R.N., 1984, SASS wind ambiguity removal by direct minimization, 2, Mon. Weather Rev., 112, 1829-1852.

Huhnerfuss, H., W. Alpers, A. Cross, W.D. Garrett, W.C. Keller, P.A. Large, W.J. Plant, F. Schlude, and D.L. Schuler, 1983, The modification of $X$ and $L$ band radar signals by monomolecular sea slicks, J. Geophys. Res., 88, 9817-9822.

Jones, W.L., L.C. Schroeder, and J.L. Mitchell, 1977, Aircraft measurements of the microwave scattering signature of the ocean, IEEE Trans. Antennas Propagat., AP-25, 52-61.

Jones, W.L., L.C. Schroeder, D.H. Boggs, E.M. Bracalente, R.A. Brown, G.J. Dome, W.J. Pierson, and F.J. Wentz, 1982, The Seasat-A Satellite Scatterometer: The geophysical evaluation of remotely sensed wind vectors over the ocean, J. Geophys. Res., 87, 3297-3317.

Keller, W.C., W.J. Plant, and D.E. Weissman, 1985, The dependence of X band microwave sea return on atmospheric stability and sea state, J. Geophys. Res., 90, 1019-1029.

Kendall, J.M., 1970, The turbulent boundary layer over a wall with progressive surface waves, J. Fluid Mech., 41, 259-281.

Kitaigorodskii, S.A., 1970, Physics of the Air-Sea Interaction, Israel Program for Scientific Translations, Ltd. 
Kitaigorodskii, S.A., 1983, On the theory of the equilibrium range in the spectrum of wind-generated gravity waves, J. Phys. Oceanogr., 13, 816-827.

Kitaigorodskii, S.A., and M.M. Zaslavskii, 1974, A dynamical analysis of the drag conditions at the sea surface, Bound. Layer Meteorol., 6, 53-61.

Kwoh, D.S.W., and B.M. Lake, 1984, A deterministic, coherent and dual-polarized laboratory study of microwave backscattering from water waves, 1, Short gravity waves without wind, IEEE J. Oceanic. Eng., OE-9(5), 291-308.

Lame, D.B., and G.H. Born, 1982, Seasat measurement system evaluation: Achievements and limitations, J. Geophys. Res., 87, 3175-3178.

Large, W.G., and S. Pond, 1981, Open ocean momentum flux measurements in moderate to strong winds, J. Phys. Oceanogr., 11, 324-336.

Leetmaa, A., and A.F. Bunker, 1978, Updated charts of the mean annual wind stress, convergences in the Ekman layers, and Sverdrup transports in the North Atlantic, J. Mar. Res., 36, 311-322.

Liu, W.T., and W.G. Large, 1981, Determination of surface stress by Seasat-SASS, J. Phys. Oceanogr., 11, 1603-1611.

Lleonart, G.T., and D.R. Blackman, 1980, The spectral characteristics of windgenerated capillary waves, J. Fluid Mech., 97, 455-479.

Longuet-Higgins, M.S., 1978, Dynamics of steep gravity waves in deep water, in Turbulent Fluxes Through the Sea Surface, Wave Dynamics and Prediction, edited by A. Favre and K. Hasselmann, Plenum, New York, 665pp.

Lyzenga, D.R., A.L. Maffett, and R.A. Shuchman, 1983, The contribution of wedge scattering to the radar cross section of the ocean surface, IEEE Trans. Geosci. Electron., GE-21, 502-505.

Miles, J.W., 1962, On the generation of surface waves by shear flows, J. Fluid Mech., 13, 433-448.

Monin, A.S., and A.M. Obukhov, 1954, Basic laws of turbulent mixing in the ground layer of the atmosphere, Akad. Nauk, SSSR Geofiz. Inst. Tr., 151, 163-187.

Moore, R.K., and A.K. Fung, 1979, Radar determination of winds at sea, Proc. IEEE, 67, 1504-1521.

Moore, R.K., and W.J. Pierson, 1967, Measuring sea state and estimating surface winds from a polar orbiting satellite, in Proceedings of the Symposium on Electromagnetic Sensing of the Earth from Satellites, edited by R. Zirkland, Polytechnic Press, New York, R1-R28.

Moore, R.K., U.S. Yu, A.K. Fung, D. Kaneko, G.J. Dome, and R.E. Werp, 1979, Preliminary study of rain effects on radar scattering from water surfaces, IEEE $J$, Oceanic Eng., OE-4(1), 31-32. 
Muller, P., and C. Frankignoul, 1981, Direct atmospheric forcing of geostrophic eddies, J. Phys. Oceanogr., 11, 287-308.

O'Brien, J.J., 1982, Scientific Opportunities Using Satellite Wind Stress Measurements Over the Ocean: Report of the Satellite Surface Stress Working Group (NOAA), Nova University/N.Y.I.T. Press, Fort Lauderdale, FL, 153pp.

Obukhov, A.M., 1946, Turbulence in an atmosphere with inhomogeneous temperature, Tr. Inst. Teor. Geofiz. Akad. Nauk. SSSR., 1, 95-115.

Peteherych, S., M.G. Wurtele, P.M. Woiceshyn, D.H. Boggs, and R.Atlas, 1984, First global analysis of Seasat scatterometer winds and potential for meterological research, Proceedings of the URSI Commission F Symposium and Workshop, Shoresh, Israel, May 14-23, 1984, NASA Conf. Publ. 2303, 575-585.

Phillips, O.M., 1977, The dynamics of the upper ocean, 2nd ed., Cambridge University Press, Cambridge, 336pp.

Phillips, O.M., 1978, Strong interaction in wind-wave fields, In Turbulent Fluxes Through the Sea Surface, Wave Dynamics and Prediction, edited by A. Favre and K. Hasselmann, Plenum, New York, 665pp.

Phillips, O.M., 1985, Spectral and statistical properties of the equilibrium range in wind-generated gravity waves, J. Fluid Mech., 156, 505-531.

Pierson, W.J., 1983, The measurement of the synoptic scale wind over the ocean, $J$. Geophys. Res., 88, 1683-1708.

Pierson, W.J., W.B. Sylvester, and M.A. Donelan, 1986, Aspects of the determination of winds by means of scatterometry and of the utilization of vector wind data for meteorological forecasts, J. Geophys. Res., 91, 2263-2272.

Plant, W.J., 1986, A two-scale model of short wind-generated waves and scatterometry, J. Geophys. Res., 91, 10735-10749.

Pond, S., G.T. Phelps, J.E. Paquin, G. McBean, and R.W. Stewart, 1971, Measurements of the turbulent fluxes of momentum, moisture, and sensible heat over the ocean, J. Atmos. Sci., 28, 901-917.

Schroeder, L.C., W.L. Grantham, J.L. Mitchell, and J.L. Sweet, 1982a, SASS measurements of the $\mathrm{K}_{\mathrm{u}}$ band radar signature of the ocean, IEEE J. Oceanic Eng., OE-7, 3-14.

Schroeder, L.C., D.H. Boggs, G.J. Dome, I.M. Halberstam, W.L. Jones, W.J. Pierson, and F.J. Wentz, 1982b, The relationship between wind vector and normalized radar cross section used to derive Seasat-A satellite scatterometer winds, J. Geophys. Res., 87, 3318-3336.

Smith, S.D., 1980, Wind stress and heat flux over the ocean in gale force winds, $J$. Phys. Oceanogr., 10, 709-726. 
Stewart, R.H., 1985, Methods of Satellite Oceanography, University of California Press, Berkeley, CA, 360pp.

Valenzuela, G.R., 1978, Theories for the interaction of electromagnetic and oceanic waves - a review, Bound. Layer Meteorol., 13, 61-85.

Veronis, G., 1981, Dynamics of large-scale ocean circulation, in Evolution of Physical Oceanography, edited by B.A. Warren and C. Wunsch, The MIT Press, Cambridge, MA, 140-183.

Wentz, F.J., S. Peteherych, and L.A. Thomas, 1984, A model function for ocean radar cross sections at $14.6 \mathrm{GHz}, J$. Geophys. Res., 89, 3689-3704.

Wentz, F.J., L.A. Mattox, and S. Peteherych, 1986, New algorithms for microwave measurements of ocean winds: Applications to Seasat and the special sensor microwave imager, J. Geophys. Res., 91, 2289-2307.

Willebrand, J., 1978, Temporal and spatial scales of the wind field over the North Pacific and North Atlantic, J. Phys. Oceanogr., 8, 1080-1094.

Willebrand, J., S.G.H. Philander, and R.C. Pacanowski, 1980, The oceanic response to large-scale atmospheric disturbances, J. Phys. Oceanogr., 10, 411-429.

Woiceshyn, P.M., M.G. Wurtele, D.H. Boggs, L.F. McGoldrick, and S. Peteherych, 1984, Proceedings of the URSI Commission F Symposium and Workshop, Shoresh, Israel, May 14-23, 1984, NASA Conf. Publ. 2303, 57-74.

Woiceshyn, P.M., M.G. Wurtele, D.H. Boggs, L.F. McGoldrick, and S. Peteherych, 1984, The necessity for a new parameterization of an empirical model for wind/ocean scatterometry, J. Geophys. Res., 91, 2273-2288.

Wright, J.W., 1968, A new model for sea clutter, IEEE Trans. Antennas Propag., AP-16, 217-223.

Wu, Jin, 1968, Laboratory studies of wind-wave interaction, J. Fluid Mech., 34, 91112.

Wu, Jin, 1980, Wind-stress coefficients over sea surface near neutral conditions - a revisit, J. Phys. Oceanogr., 10, 727-740.

Wurtele, M.G., P.M. Woiceshyn, S. Peteherych, M. Borowski, and W.S. Appleby, 1982, Wind direction alias removal studies of Seasat scatterometer-derived wind fields, J. Geophys. Res., 87, 3365-3377.

Yu, T.W., and R.D. McPherson, 1984, Global data assimilation experiments with scatterometer winds from Seasat-A, Mon. Weather Rev., 112, 368-376. 
Table 1: Ungridded Atlas SASS component wind statistics Individual area averages

U COMPONENT

\begin{tabular}{|c|c|c|c|c|c|c|}
\hline \multicolumn{2}{|c|}{ AREA } & $\begin{array}{l}\text { MEAN } \\
(\mathrm{m} / \mathrm{s})\end{array}$ & $\begin{array}{c}\text { VAR } \\
\left(m^{\wedge} 2 / s^{\wedge} 2\right)\end{array}$ & WVAR & $\begin{array}{l}\text { SDEV } \\
(\mathrm{m} / \mathrm{s})\end{array}$ & $\mathrm{N}$ \\
\hline \multirow{2}{*}{658} & $\mathrm{U1}$ & 2.239 & 12.195 & 0.715 & 3.492 & 76 \\
\hline & $\mathrm{U} 2$ & -3.163 & 10.170 & 0.507 & 3.189 & 76 \\
\hline \multirow[t]{3}{*}{664} & $\mathrm{U} 1$ & -6.794 & 5.514 & 0.107 & 2.348 & 75 \\
\hline & $\mathrm{U} 2$ & -3.700 & 12.953 & 0.489 & 3.599 & 99 \\
\hline & U3 & 2.241 & 21.700 & 0.821 & 4.658 & 71 \\
\hline 665 & U1 & -1.158 & 9.218 & 0.883 & 3.036 & 76 \\
\hline \multirow{3}{*}{672} & u1 & 5.543 & 8.045 & 0.208 & 2.836 & 61 \\
\hline & U2 & 3.894 & 7.466 & 0.331 & 2.732 & 77 \\
\hline & U3 & -4.825 & 1.053 & 0.043 & 1.026 & 48 \\
\hline 678 & U1 & -1.634 & 5.265 & 0.671 & 2.295 & 61 \\
\hline \multirow[t]{2}{*}{679} & U1 & -6.254 & 5.689 & 0.127 & 2.385 & 72 \\
\hline & U2 & -1.521 & 28.067 & 0.934 & 5.298 & 85 \\
\hline \multirow[t]{2}{*}{686} & U1 & -3.978 & 11.816 & 0.430 & 3.437 & 75 \\
\hline & U2 & -7.641 & 4.322 & 0.069 & 2.079 & 114 \\
\hline 692 & U1 & -2.129 & 10.841 & 0.717 & 3.293 & 43 \\
\hline \multirow[t]{3}{*}{693} & U1 & -6.517 & 11.607 & 0.215 & 3.407 & 120 \\
\hline & $\mathrm{U} 2$ & -3.370 & 19.035 & 0.632 & 4.363 & 74 \\
\hline & U3 & 4.943 & 12.997 & 0.349 & 3.605 & 65 \\
\hline 700 & UI & -4.808 & 5.481 & 0.192 & 2.341 & 113 \\
\hline \multirow[t]{3}{*}{701} & U1 & 4.454 & 2.980 & 0.131 & 1.726 & 59 \\
\hline & U2 & 1.181 & 16.580 & 0.935 & 4.072 & 71 \\
\hline & U3 & -4.342 & 18.507 & 0.500 & 4.302 & 56 \\
\hline \multirow{2}{*}{708} & U1 & -2.730 & 22.909 & 0.764 & 4.786 & 61 \\
\hline & U2 & 2.348 & 11.282 & 0.679 & 3.359 & 61 \\
\hline \multirow[t]{4}{*}{715} & U1 & 11.136 & 13.919 & 0.101 & 3.731 & 80 \\
\hline & U2 & 8.567 & 11.062 & 0.131 & 3.326 & 88 \\
\hline & U3 & 2.072 & 37.505 & 0.909 & 6.124 & 70 \\
\hline & $\mathrm{U} 4$ & -3.123 & 18.114 & 0.655 & 4.256 & 88 \\
\hline 716 & U1 & 2.460 & 4.923 & 0.456 & 2.219 & 27 \\
\hline 721 & U1 & 4.859 & 12.490 & 0.348 & 3.534 & 71 \\
\hline \multirow[t]{2}{*}{722} & $\mathrm{U} 1$ & -4.467 & 25.776 & 0.568 & 5.077 & 68 \\
\hline & U2 & 1.734 & 21.393 & 0.890 & 4.625 & 61 \\
\hline \multirow[t]{2}{*}{729} & U1 & 1.124 & 19.738 & 0.951 & 4.443 & 8 \\
\hline & U2 & -4.625 & 8.739 & 0.291 & 2.956 & 83 \\
\hline \multirow{4}{*}{$\begin{array}{l}135 \\
736\end{array}$} & U1 & 10.240 & 15.319 & 0.128 & 3.914 & 27 \\
\hline & UI & -3.009 & 21.125 & 0.704 & 4.596 & 120 \\
\hline & U2 & 0.722 & 6.218 & 0.934 & 2.494 & 7. \\
\hline & U3 & 0.762 & 6.879 & 0.940 & 2.623 & 45 \\
\hline \multirow[t]{3}{*}{744} & $\mathrm{U} 1$ & 2.192 & 8.751 & 0.652 & 2.958 & 68 \\
\hline & U2 & 1.252 & 11.277 & 0.883 & 3.358 & 171 \\
\hline & v3 & -3.020 & 25.656 & 0.741 & 5.065 & 15 \\
\hline \multirow[t]{3}{*}{750} & U1 & -3.405 & 18.457 & 0.617 & 4.296 & 135 \\
\hline & U2 & -0.481 & 8.251 & 0.980 & 2.872 & 127 \\
\hline & U3 & 0.055 & 12.184 & 1.009 & 3.491 & 115 \\
\hline 751 & U1 & 0.062 & 28.627 & 1.008 & 5.350 & 12 \\
\hline 758 & UI & 3.504 & 5.505 & 0.311 & 2.346 & 7 \\
\hline 772 & U1 & 3.671 & 6.040 & 0.311 & 2.458 & 6 \\
\hline 773 & U1 & 1.280 & 2.570 & 0.621 & 1.603 & 3 \\
\hline \multirow[t]{2}{*}{779} & U1 & -4.380 & 8.037 & 0.296 & 2.835 & 125 \\
\hline & $\mathrm{U} 2$ & 1.420 & 31.006 & 0.945 & 5.568 & 14 \\
\hline
\end{tabular}




\begin{tabular}{|c|c|c|c|c|c|c|}
\hline & U3 & 5.308 & 11.379 & 0.289 & 3.373 & 72 \\
\hline 780 & U1 & -5.037 & 9.672 & 0.278 & 3.110 & 39 \\
\hline 787 & U1 & 3.320 & 12.649 & 0.538 & 3.557 & 86 \\
\hline & U2 & 0.965 & 48.947 & 0.992 & 6.996 & 92 \\
\hline & u3 & 3.462 & 18.393 & 0.608 & 4.289 & 151 \\
\hline & U4 & -3.216 & 27.771 & 0.733 & 5.270 & 115 \\
\hline 793 & U1 & 2.559 & 12.247 & 0.656 & 3.500 & 88 \\
\hline & บ2 & -0.043 & 10.198 & 1.015 & 3.193 & 65 \\
\hline 794 & $\mathrm{U} 1$ & -3.326 & 31.193 & 0.747 & 5.585 & 61 \\
\hline & U2 & 4.334 & 25.594 & 0.582 & 5.059 & 68 \\
\hline 801 & UI & 2.201 & 18.236 & 0.796 & 4.270 & 98 \\
\hline & $\mathrm{U} 2$ & -0.016 & 33.640 & 1.009 & 5.800 & 116 \\
\hline & U3 & 2.832 & 8.416 & 0.514 & 2.901 & 153 \\
\hline & U4 & -1.722 & 11.532 & 0.801 & 3.396 & 118 \\
\hline 802 & U1 & 1.927 & 21.812 & 0.865 & 4.670 & 68 \\
\hline & U2 & 1.702 & 33.717 & 0.937 & 5.807 & 53 \\
\hline 807 & U1 & -1.112 & 35.657 & 0.992 & 5.971 & 38 \\
\hline 808 & U1 & -2.619 & 16.112 & 0.706 & 4.014 & 117 \\
\hline & $\mathrm{U} 2$ & -0.460 & 12.382 & 0.993 & 3.519 & 100 \\
\hline & U3 & 0.987 & 8.601 & 0.914 & 2.933 & 53 \\
\hline 815 & U1 & -5.708 & 2.140 & 0.062 & 1.463 & 59 \\
\hline 816 & U1 & -0.979 & 59.283 & 1.000 & 7.700 & 63 \\
\hline & $\mathrm{U} 2$ & 0.640 & 22.835 & 0.989 & 4.779 & 158 \\
\hline & U3 & -0.520 & 19.534 & 0.995 & 4.420 & 113 \\
\hline 822 & $\mathrm{U} 1$ & -4.443 & 7.856 & 0.285 & 2.803 & 113 \\
\hline & $\mathrm{U} 2$ & 0.958 & 21.369 & 0.965 & 4.623 & 139 \\
\hline & U3 & 4.553 & 11.921 & 0.366 & 3.453 & 134 \\
\hline 823 & U1 & -0.394 & 18.463 & 1.008 & 4.297 & 61 \\
\hline 830 & U1 & 4.266 & 32.819 & 0.646 & 5.729 & 141 \\
\hline & $\mathrm{U} 2$ & 5.458 & 12.738 & 0.300 & 3.569 & 158 \\
\hline & U3 & 0.741 & 10.356 & 0.956 & 3.218 & 143 \\
\hline 836 & U1 & 6.032 & 10.190 & 0.219 & 3.192 & 87 \\
\hline & U2 & 3.525 & 48.995 & 0.806 & 7.000 & 80 \\
\hline 837 & $\mathrm{U} 1$ & -1.658 & 6.134 & 0.695 & 2.477 & 100 \\
\hline 844 & U1 & 4.147 & 4.534 & 0.209 & 2.129 & 94 \\
\hline & $\mathrm{U} 2$ & -1.012 & 32.243 & 0.977 & 5.678 & 130 \\
\hline 845 & U1 & 2.000 & 20.368 & 0.843 & 4.513 & 93 \\
\hline 865 & U1 & -2.916 & 20.489 & 0.710 & 4.526 & 150 \\
\hline & $\mathrm{U} 2$ & 1.010 & 25.822 & 0.967 & 5.082 & 185 \\
\hline & U3 & -1.217 & 54.924 & 0.982 & 7.411 & 120 \\
\hline 865 & U4 & -2.385 & 55.281 & 0.915 & 7.435 & 101 \\
\hline 866 & U1 & 2.522 & 7.549 & 0.548 & 2.748 & 63 \\
\hline 873 & U1 & -1.980 & 38.363 & 0.917 & 6.194 & 86 \\
\hline & $\mathrm{U} 2$ & 2.679 & 21.807 & 0.759 & 4.670 & 81 \\
\hline & U3 & -0.835 & 24.610 & 0.981 & 4.961 & 109 \\
\hline 887 & $\mathrm{U} 1$ & 0.512 & 11.762 & 0.987 & 3.430 & 109 \\
\hline & $\mathrm{U} 2$ & -3.158 & 11.422 & 0.537 & 3.380 & 104 \\
\hline 888 & U1 & -3.753 & 2.759 & 0.164 & 1.661 & 44 \\
\hline 901 & U1 & -3.811 & 11.514 & 0.444 & 3.393 & 138 \\
\hline 902 & U1 & -2.939 & 7.677 & 0.474 & 2.771 & 63 \\
\hline & U2 & -3.200 & 15.885 & 0.612 & 3.986 & 107 \\
\hline 916 & U1 & -0.979 & 26.278 & 0.978 & 5.126 & 72 \\
\hline 916 & $\mathrm{U} 2$ & -3.696 & 17.048 & 0.559 & 4.129 & 83 \\
\hline 916 & U3 & -3.876 & 8.983 & 0.376 & 2.997 & 103 \\
\hline 930 & U1 & -1.091 & 7.981 & 0.880 & 2.825 & 78 \\
\hline 930 & U2 & -4.647 & 5.679 & 0.209 & 2.383 & 102 \\
\hline 951 & U1 & -2.033 & 18.650 & 0.827 & 4.319 & 76 \\
\hline 951 & U2 & 1.033 & 4.710 & 0.824 & 2.170 & 78 \\
\hline
\end{tabular}




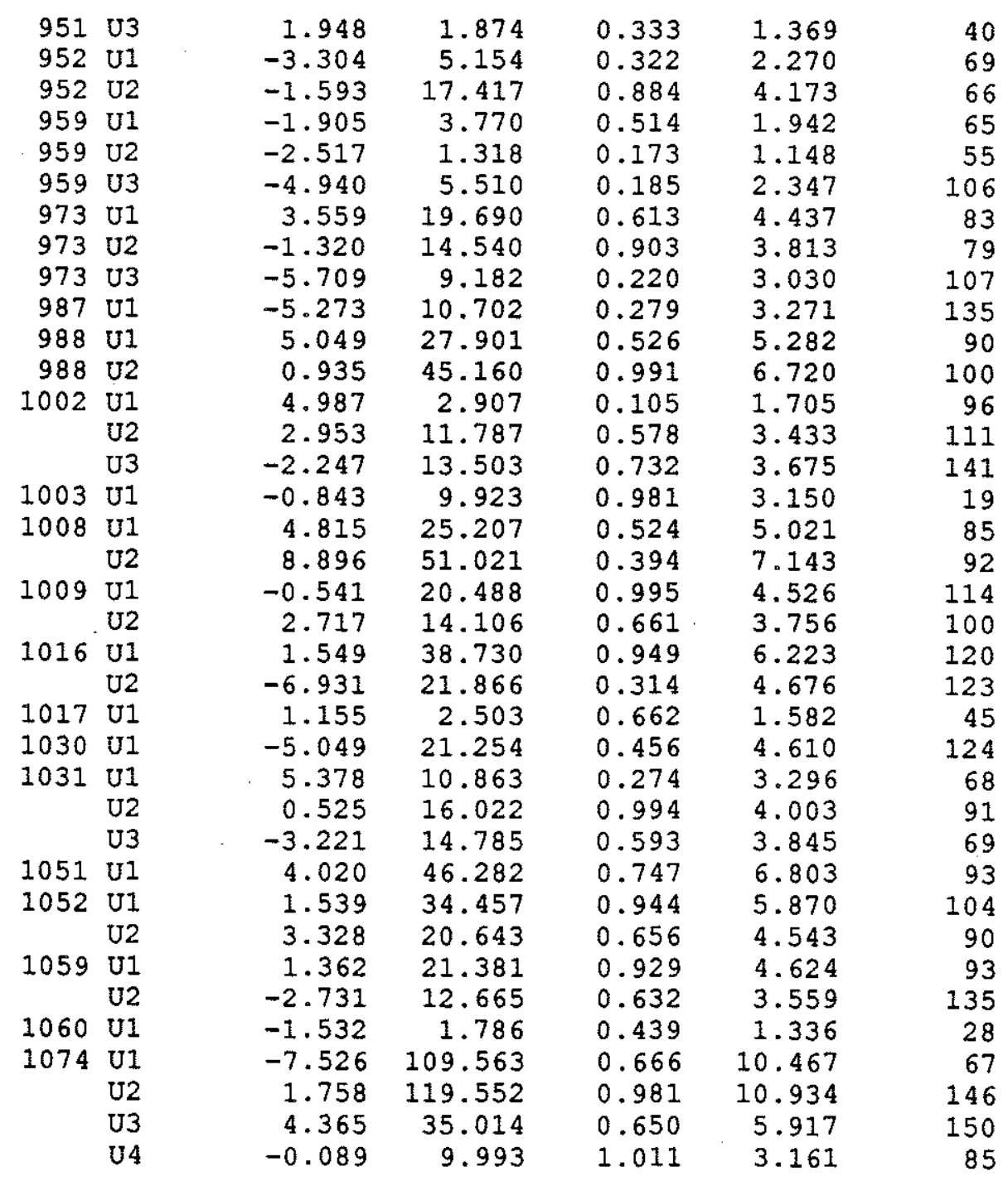

$\mathrm{V}$ COMPONENT

\begin{tabular}{llrrrrr} 
AREA & \multicolumn{1}{c}{$\begin{array}{c}\text { MEAN } \\
(\mathrm{m} / \mathrm{s})\end{array}$} & \multicolumn{1}{c}{$\begin{array}{c}\text { VAR } \\
\left(\mathrm{m}^{\wedge} 2 / \mathrm{s}^{\wedge} 2\right)\end{array}$} & WVAR & $\begin{array}{c}\text { SDEV } \\
(\mathrm{m} / \mathrm{s})\end{array}$ & N \\
658 V1 & 2.239 & 12.195 & 0.715 & 3.492 & 76 \\
& V2 & 1.113 & 2.851 & 0.704 & 1.688 & 76 \\
664 & V1 & 0.611 & 7.517 & 0.965 & 2.742 & 75 \\
& V2 & 1.147 & 5.766 & 0.821 & 2.401 & 99 \\
& V3 & 2.828 & 11.097 & 0.586 & 3.331 & 71 \\
665 & V1 & 5.400 & 16.441 & 0.362 & 4.055 & 76 \\
672 V1 & 3.642 & 7.809 & 0.373 & 2.795 & 61 \\
& V2 & 3.984 & 5.697 & 0.265 & 2.387 & 77 \\
& V3 & 0.099 & 1.588 & 1.015 & 1.260 & 48 \\
678 V1 & 3.176 & 4.131 & 0.292 & 2.033 & 61 \\
679 & V1 & 0.843 & 3.930 & 0.857 & 1.982 & 72 \\
& V2 & 2.635 & 11.560 & 0.629 & 3.400 & 85 \\
686 V1 & -1.751 & 5.423 & 0.644 & 2.329 & 75 \\
& V2 & -1.094 & 5.421 & 0.825 & 2.328 & 114
\end{tabular}




\begin{tabular}{|c|c|c|c|c|c|c|}
\hline 692 & V1 & 3.860 & 7.561 & 0.339 & 2.750 & 43 \\
\hline 693 & V1 & 0.839 & 10.328 & 0.944 & 3.214 & 120 \\
\hline & $\mathrm{V} 2$ & 1.690 & 6.960 & 0.716 & 2.638 & 74 \\
\hline & V3 & 4.355 & 5.566 & 0.228 & 2.359 & 65 \\
\hline 700 & V1 & -4.140 & 2.101 & 0.109 & 1.450 & 113 \\
\hline 701 & $\mathrm{VI}$ & 0.036 & 11.975 & 1.017 & 3.461 & 59 \\
\hline & $\mathrm{V} 2$ & 1.234 & 8.679 & 0.861 & 2.946 & 71 \\
\hline & v3 & 1.110 & 4.054 & 0.778 & 2.014 & 56 \\
\hline 708 & V1 & 1.349 & 5.311 & 0.754 & 2.305 & 61 \\
\hline & $\mathrm{V} 2$ & 1.418 & 5.500 & 0.741 & 2.345 & 61 \\
\hline 715 & V1 & -0.836 & 50.865 & 0.999 & 7.132 & 80 \\
\hline & V2 & -1.705 & 13.122 & 0.826 & 3.622 & 88 \\
\hline & v3 & -0.074 & 1.749 & 1.011 & 1.323 & 70 \\
\hline & V4 & -0.891 & 10.667 & 0.941 & 3.266 & 88 \\
\hline 716 & V1 & 2.668 & 3.282 & 0.319 & 1.812 & 27 \\
\hline 721 & V1 & -2.641 & 16.314 & 0.708 & 4.039 & 71 \\
\hline 722 & V1 & -0.276 & 8.851 & 1.006 & 2.975 & 68 \\
\hline & $\mathrm{V} 2$ & 0.317 & 9.734 & 1.006 & 3.120 & 61 \\
\hline 729 & $\mathrm{~V} 1$ & -1.426 & 3.562 & 0.641 & 1.887 & 84 \\
\hline & V2 & -1.527 & 3.794 & 0.624 & 1.948 & 83 \\
\hline 735 & VI & -0.296 & 9.079 & 1.028 & 3.013 & 27 \\
\hline 736 & $\mathrm{VI}$ & -0.962 & 4.923 & 0.848 & 2.219 & 120 \\
\hline & V2 & -1.499 & 4.757 & 0.686 & 2.181 & 74 \\
\hline & V3 & 4.016 & 2.863 & 0.151 & 1.692 & 49 \\
\hline 744 & V1 & 2.106 & 7.554 & 0.636 & 2.749 & 68 \\
\hline & V2 & 3.453 & 9.689 & 0.449 & 3.113 & 171 \\
\hline & V3 & 1.720 & 8.680 & 0.749 & 2.946 & 151 \\
\hline 750 & V1 & -1.213 & 4.194 & 0.744 & 2.048 & 135 \\
\hline & V2 & -0.006 & 8.246 & 1.008 & 2.872 & 127 \\
\hline & v3 & 1.599 & 9.527 & 0.794 & 3.087 & 115 \\
\hline 751 & V1 & 3.249 & 7.272 & 0.409 & 2.697 & 126 \\
\hline 758 & VI & 2.377 & 6.555 & 0.541 & 2.560 & 79 \\
\hline 772 & V1 & 3.604 & 3.844 & 0.229 & 1.960 & 61 \\
\hline 773 & $\mathrm{~V} 1$ & -0.583 & 5.309 & 0.965 & 2.304 & 36 \\
\hline 779 & V1 & 0.430 & 4.323 & 0.966 & 2.079 & 125 \\
\hline & V2 & 2.459 & 11.457 & 0.658 & 3.385 & 146 \\
\hline & V3 & 1.066 & 45.594 & 0.989 & 6.752 & 72 \\
\hline 780 & V1 & 2.732 & 6.250 & 0.461 & 2.500 & 39 \\
\hline 787 & V1 & -2.476 & 50.567 & 0.901 & 7.111 & 86 \\
\hline & $\mathrm{V} 2$ & 2.470 & 14.157 & 0.704 & 3.763 & 92 \\
\hline & V3 & 2.505 & 9.000 & 0.592 & 3.000 & 151 \\
\hline & V4 & 1.879 & 4.599 & 0.569 & 2.144 & 115 \\
\hline 793 & VI & -4.761 & 5.758 & 0.203 & 2.400 & 88 \\
\hline & $\mathrm{V} 2$ & -4.884 & 11.843 & 0.333 & 3.441 & 65 \\
\hline 794 & VI & 2.685 & 8.247 & 0.538 & 2.872 & 61 \\
\hline & V2 & -0.189 & 13.089 & 1.012 & 3.618 & 68 \\
\hline 801 & V1 & -6.007 & 20.076 & 0.359 & 4.481 & 98 \\
\hline & V2 & 1.841 & 18.428 & 0.851 & 4.293 & 116 \\
\hline & V3 & 0.232 & 17.031 & 1.003 & 4.127 & 153 \\
\hline & V4 & -1.083 & 6.999 & 0.863 & 2.645 & 118 \\
\hline 802 & V1 & -3.434 & 22.533 & 0.663 & 4.747 & 68 \\
\hline & V2 & 1.165 & 5.231 & 0.806 & 2.287 & 53 \\
\hline 807 & V1 & 0.762 & 9.377 & 0.966 & 3.062 & 38 \\
\hline 808 & V1 & 1.674 & 8.586 & 0.759 & 2.930 & 117 \\
\hline & V2 & 0.878 & 19.249 & 0.971 & 4.387 & 100 \\
\hline & V3 & -3.971 & 26.968 & 0.639 & 5.193 & 53 \\
\hline 815 & VI & -1.284 & 3.451 & 0.685 & 1.858 & 59 \\
\hline 816 & V1 & -0.630 & 16.429 & 0.992 & 4.053 & 63 \\
\hline
\end{tabular}




\begin{tabular}{|c|c|c|c|c|c|c|}
\hline & v2 & -1.613 & 20.813 & 0.894 & 4.562 & 158 \\
\hline & v3 & 1.754 & 14.067 & 0.826 & 3.751 & 113 \\
\hline \multirow[t]{3}{*}{822} & V1 & 0.627 & 3.082 & 0.894 & 1.756 & 113 \\
\hline & v2 & 1.909 & 4.986 & 0.580 & 2.233 & 139 \\
\hline & v3 & 1.915 & 21.302 & 0.859 & 4.615 & 134 \\
\hline 823 & v1 & 0.008 & 21.622 & 1.017 & 4.650 & 61 \\
\hline \multirow[t]{3}{*}{830} & V1 & 1.655 & 23.899 & 0.903 & 4.889 & 141 \\
\hline & v2 & 2.108 & 15.136 & 0.777 & 3.890 & 158 \\
\hline & v3 & 2.654 & 4.139 & 0.371 & 2.034 & 143 \\
\hline \multirow[t]{2}{*}{836} & V1 & 0.470 & 5.014 & 0.969 & 2.239 & 87 \\
\hline & v2 & 5.145 & 21.086 & 0.446 & 4.592 & 80 \\
\hline 837 & v1 & 1.111 & 11.967 & 0.915 & 3.459 & 100 \\
\hline \multirow[t]{2}{*}{844} & V1 & -1.421 & 3.592 & 0.645 & 1.895 & 94 \\
\hline & v2 & -0.865 & 4.108 & 0.851 & 2.027 & 130 \\
\hline 845 & V1 & -1.601 & 7.858 & 0.760 & 2.803 & 93 \\
\hline \multirow[t]{4}{*}{865} & v1 & 3.057 & 12.302 & 0.570 & 3.507 & 150 \\
\hline & v2 & 3.801 & 15.677 & 0.522 & 3.959 & 185 \\
\hline & V3 & 4.349 & 11.032 & 0.370 & 3.321 & 120 \\
\hline & V4 & 1.899 & 37.834 & 0.921 & 6.151 & 101 \\
\hline \multirow{4}{*}{$873^{\circ}$} & VI & -1.374 & 14.778 & 0.899. & 3.844 & 63 \\
\hline & VI & 3.084 & 31.995 & 0.778 & 5.656 & 86 \\
\hline & v2 & 6.051 & 6.499 & 0.151 & 2.549 & 81 \\
\hline & v3 & 4.083 & 6.279 & 0.274 & 2.506 & 109 \\
\hline \multirow[t]{2}{*}{887} & V1 & 3.891 & 8.459 & 0.360 & 2.908 & 109 \\
\hline & v2 & 0.561 & 8.054 & 0.971 & 2.838 & 104 \\
\hline 888 & v1 & 0.728 & 0.862 & 0.628 & 0.928 & 44 \\
\hline 901 & VI & 1.441 & 5.748 & 0.739 & 2.397 & 138 \\
\hline 902 & V1 & 4.015 & 17.281 & 0.522 & 4.157 & 63 \\
\hline 902 & V2 & 0.665 & 4.284 & 0.914 & 2.070 & 107 \\
\hline 916 & V1 & -6.505 & 14.878 & 0.261 & 3.857 & 72 \\
\hline 916 & v2 & -2.881 & 15.688 & 0.659 & 3.961 & 83 \\
\hline 916 & v3 & 1.036 & 4.868 & 0.826 & 2.206 & 103 \\
\hline 930 & V1 & -3.435 & 14.218 & 0.550 & 3.771 & 78 \\
\hline 930 & v2 & -0.453 & 5.487 & 0.973 & 2.342 & 102 \\
\hline 951 & v1 & -2.081 & 2.353 & 0.354 & 1.534 & 76 \\
\hline 951 & V2 & -2.459 & 3.180 & 0.346 & 1.783 & 78 \\
\hline 951 & v3 & -2.849 & 3.188 & 0.284 & 1.785 & 40 \\
\hline 952 & VI & 7.529 & 17.104 & 0.233 & 4.136 & 69 \\
\hline 952 & v2 & 7.056 & 21.487 & 0.303 & 4.635 & 66 \\
\hline 959 & V1 & 0.807 & 10.156 & 0.954 & 3.187 & 65 \\
\hline 959 & v2 & 0.621 & 4.453 & 0.936 & 2.110 & 55 \\
\hline 959 & v3 & -3.832 & 7.200 & 0.330 & 2.683 & 106 \\
\hline 973 & V1 & -1.431 & 6.471 & 0.767 & 2.544 & 83 \\
\hline 973 & v2 & -2.755 & 2.635 & 0.259 & 1.623 & 79 \\
\hline 973 & v3 & -1.309 & 4.365 & 0.723 & 2.089 & 107 \\
\hline 987 & V1 & -2.892 & 4.494 & 0.350 & 2.120 & 135 \\
\hline 988 & V1 & 8.259 & 39.916 & 0.371 & 6.318 & 90 \\
\hline 988 & v2 & 3.847 & 12.100 & 0.452 & 3.479 & 100 \\
\hline \multirow[t]{3}{*}{1002} & V1 & 9.504 & 3.795 & 0.040 & 1.948 & 96 \\
\hline & v2 & 6.334 & 13.369 & 0.250 & 3.656 & 111 \\
\hline & V3 & 1.992 & 5.411 & 0.579 & 2.326 & 141 \\
\hline 1003 & V1 & -4.514 & 5.538 & 0.216 & 2.353 & 19 \\
\hline \multirow[t]{2}{*}{1008} & $\mathrm{~V} 1$ & 6.589 & 21.260 & 0.330 & 4.611 & 85 \\
\hline & v2 & 9.472 & 51.634 & 0.367 & 7.186 & 92 \\
\hline \multirow[t]{2}{*}{1009} & v1 & 2.303 & 4.063 & 0.435 & 2.016 & 114 \\
\hline & v2 & 0.846 & 9.730 & 0.940 & 3.119 & 100 \\
\hline \multirow[t]{2}{*}{1016} & V1 & 5.922 & 10.729 & 0.235 & 3.276 & 120 \\
\hline & V2 & 1.466 & 24.592 & 0.927 & 4.959 & 123 \\
\hline
\end{tabular}




$\begin{array}{rlrrrrr}1017 & \text { V1 } & 5.186 & 7.805 & 0.226 & 2.794 & 45 \\ 1030 & \text { V1 } & -1.957 & 8.104 & 0.683 & 2.847 & 124 \\ 1031 & \text { V1 } & 3.343 & 9.739 & 0.469 & 3.121 & 68 \\ & \text { V2 } & 1.189 & 14.765 & 0.922 & 3.843 & 91 \\ \text { V3 } & -1.570 & 24.552 & 0.921 & 4.955 & 69 \\ 1051 & \text { V1 } & 5.661 & 108.102 & 0.778 & 10.397 & 93 \\ 1052 \text { V1 } & 0.507 & 30.187 & 1.001 & 5.494 & 104 \\ & \text { V2 } & -4.635 & 28.670 & 0.575 & 5.354 & 90 \\ 1059 & \text { V1 } & 7.275 & 12.706 & 0.194 & 3.565 & 93 \\ \text { V2 } & 5.064 & 9.957 & 0.280 & 3.155 & 135 \\ 1060 & \text { V1 } & -1.663 & 2.368 & 0.469 & 1.539 & 28 \\ 1074 & \text { V1 } & 5.256 & 81.280 & 0.755 & 9.016 & 67 \\ \text { V2 } & -1.240 & 121.694 & 0.994 & 11.031 & 146 \\ & \text { V3 } & -0.991 & 67.082 & 0.992 & 8.190 & 150 \\ \text { V4 } & -0.335 & 7.564 & 0.997 & 2.750 & 85\end{array}$


Table 2: Ungridded Atlas SASS component wind statistics - 1-day averages

\begin{tabular}{|c|c|c|c|c|c|}
\hline DAY & $\frac{M E A N}{(m / s)}$ & $\frac{\text { VAR }}{\left(\mathrm{m}^{2} / \mathrm{s}^{2}\right)}$ & WVAR & $\frac{\text { SDEV }}{(\mathrm{m} / \mathrm{s})}$ & $\underline{N}$ \\
\hline $224 \mathrm{U}$ & -0.720 & 26.060 & 0.982 & 5.105 & 486 \\
\hline V & 2.236 & 10.957 & 0.688 & 3.310 & 486 \\
\hline $225 \mathrm{U}$ & -1.897 & 32.461 & 0.902 & 5.697 & 489 \\
\hline $\mathrm{V}$ & 1.049 & 10.627 & 0.908 & 3.260 & 489 \\
\hline $226 \mathrm{U}$ & -2.776 & 32.242 & 0.808 & 5.678 & 628 \\
\hline V & -0.204 & 12.890 & 0.998 & 3.590 & 628 \\
\hline $227 \mathrm{U}$ & 0.611 & 45.880 & 0.994 & 6.773 & 539 \\
\hline V & -0.789 & 16.347 & 0.965 & 4.043 & 539 \\
\hline $228 \mathrm{U}$ & 2.117 & 45.433 & 0.912 & 6.740 & 556 \\
\hline $\mathrm{V}$ & -1.102 & 16.523 & 0.933 & 4.065 & 556 \\
\hline $229 \mathrm{U}$ & -0.322 & 29.390 & 0.998 & 5.421 & 632 \\
\hline V & 0.809 & 10.624 & 0.943 & 3.259 & 632 \\
\hline $230 \mathrm{U}$ & -0.375 & 23.460 & 0.995 & 4.844 & 724 \\
\hline V & 1.828 & 10.026 & 0.751 & 3.166 & 724 \\
\hline $231 \mathrm{U}$ & 3.052 & 6.765 & 0.422 & 2.601 & 179 \\
\hline V & 2.170 & 7.517 & 0.617 & 2.742 & 179 \\
\hline $232 \mathrm{U}$ & 0.113 & 32.800 & 1.001 & 5.727 & 634 \\
\hline V & 1.037 & 19.621 & 0.949 & 4.430 & 634 \\
\hline $233 \mathrm{U}$ & 0.012 & 30.609 & 1.001 & 5.533 & 926 \\
\hline V & -1.101 & 25.519 & 0.956 & 5.052 & 926 \\
\hline $234 \mathrm{U}$ & -1.103 & 25.662 & 0.956 & 5.066 & 869 \\
\hline V & -1.076 & 23.019 & 0.953 & 4.798 & 869 \\
\hline $235 \mathrm{U}$ & 0.316 & 29.565 & 0.998 & 5.437 & 877 \\
\hline V & 0.925 & 16.703 & 0.952 & 4.087 & 877 \\
\hline $236 \mathrm{U}$ & 1.680 & 27.300 & 0.907 & 5.225 & 810 \\
\hline V & 0.936 & 15.692 & 0.948 & 3.961 & 810 \\
\hline $237 \mathrm{U}$ & 0.970 & 26.786 & 0.969 & 5.176 & 277 \\
\hline V & -1.369 & 7.525 & 0.803 & 2.743 & 277 \\
\hline $238 \mathrm{U}$ & -1.435 & 33.986 & 0.944 & 5.830 & 561 \\
\hline V & 2.623 & 21.113 & 0.755 & 4.595 & 561 \\
\hline $239 \mathrm{U}$ & -1.515 & 21.785 & 0.907 & 4.667 & 425 \\
\hline V & 2.966 & 14.645 & 0.626 & 3.827 & 425 \\
\hline $240 \mathrm{U}$ & -2.549 & 14.509 & 0.692 & 3.809 & 504 \\
\hline V & 1.997 & 10.473 & 0.725 & 3.236 & 504 \\
\hline $241 \mathrm{U}$ & -3.267 & 15.535 & 0.593 & 3.941 & 467 \\
\hline V & 0.178 & 17.292 & 1.000 & 4.158 & 467 \\
\hline $242 \mathrm{U}$ & -2.858 & 17.301 & 0.681 & 4.159 & 363 \\
\hline V & -2.144 & 17.253 & 0.791 & 4.154 & 363 \\
\hline
\end{tabular}




$\begin{array}{rlllll}243 \mathrm{U} & -2.497 & 15.265 & 0.713 & 3.907 & 169 \\ \mathrm{~V} & -2.421 & 12.829 & 0.689 & 3.582 & 169 \\ 244 \mathrm{U} & -2.476 & 12.786 & 0.677 & 3.576 & 396 \\ \mathrm{~V} & -0.019 & 24.092 & 1.003 & 4.908 & 396 \\ 245 \mathrm{U} & -2.399 & 25.893 & 0.820 & 5.089 & 371 \\ \mathrm{~V} & -1.218 & 11.975 & 0.892 & 3.461 & 371 \\ 246 \mathrm{U} & -1.492 & 39.713 & 0.949 & 6.302 & 456 \\ \mathrm{~V} & 0.741 & 29.367 & 0.984 & 5.419 & 456 \\ 247 \mathrm{U} & -0.710 & 31.102 & 0.986 & 5.577 & 523 \\ \mathrm{~V} & 2.888 & \mathbf{3 4 . 0 8 6} & 0.805 & 5.838 & 523 \\ 248 \mathrm{U} & 0.805 & 41.735 & 0.986 & 6.460 & 765 \\ \mathrm{~V} & 4.175 & 28.833 & 0.624 & 5.370 & 765 \\ 249 \mathrm{U} & -1.746 & 40.097 & 0.931 & 6.332 & 505 \\ \mathrm{~V} & 1.782 & 26.404 & 0.894 & 5.138 & 505 \\ 250 \mathrm{U} & -1.885 & 34.931 & 0.911 & 5.910 & 266 \\ \mathrm{~V} & -0.397 & 18.012 & 0.995 & 4.244 & 266 \\ 251 \mathrm{U} & 1.109 & 29.008 & 0.961 & 5.386 & \mathbf{5 1 0} \\ \mathrm{V} & 3.620 & 53.994 & 0.806 & 7.348 & 510 \\ 252 \mathrm{U} & 0.184 & 51.466 & 1.001 & 7.174 & 474 \\ \mathrm{~V} & 2.454 & 62.263 & 0.914 & 7.891 & 474\end{array}$


Table 3: Mapped Atlas SASS component wind statistics - area averages

U component

\begin{tabular}{|c|c|c|c|c|}
\hline REV & $\frac{\text { MEAN }}{(\mathrm{m} / \mathrm{s})}$ & $\frac{\text { VAR }}{\left(\mathrm{m}^{2} / \mathrm{s}^{2}\right)}$ & WVAR & $\frac{\text { SDEV }}{(\mathrm{m} / \mathrm{s})}$ \\
\hline $658 \mathrm{U} 1$ & 4.098 & 3.378 & 0.168 & 1.838 \\
\hline U2 & -4.553 & 2.720 & 0.116 & 1.649 \\
\hline $664 \mathrm{U} 1$ & -7.179 & 3.022 & 0.055 & 1.738 \\
\hline U2 & -4.128 & 0.864 & $0: 048$ & 0.930 \\
\hline U3 & 5.628 & 14.099 & 0.311 & 3.755 \\
\hline 665 U1 & -0.152 & 3.945 & 1.022 & 1.986 \\
\hline 672 U1 & 6.097 & 4.069 & 0.099 & 2.017 \\
\hline U2 & 4.340 & 2.850 & 0.132 & 1.688 \\
\hline U3 & -5.128 & 0.858 & 0.032 & 0.926 \\
\hline $678 \mathrm{U} 1$ & -1.757 & 2.174 & 0.417 & 1.475 \\
\hline $679 \mathrm{U} 1$ & -7.142 & 1.662 & 0.032 & 1.289 \\
\hline U2 & -1.396 & 21.771 & 0.942 & 4.666 \\
\hline 686 U1 & -3.526 & 1.159 & 0.085 & 1.077 \\
\hline U2 & -8.387 & 1.265 & 0.018 & 1.125 \\
\hline $692 \mathrm{U} 1$ & -2.764 & 6.509 & 0.466 & 2.551 \\
\hline $693 \mathrm{U} 1$ & -7.849 & 4.805 & 0.072 & 2.192 \\
\hline U2 & -0.125 & 4.279 & 1.025 & 2.069 \\
\hline U3 & 7.091 & 2.947 & 0.055 & 1.717 \\
\hline $700 \mathrm{U} 1$ & -4.162 & 2.910 & 0.144 & 1.706 \\
\hline $701 \mathrm{U1}$ & 5.221 & 1.918 & 0.066 & 1.385 \\
\hline U2 & 2.582 & 3.948 & 0.376 & 1.987 \\
\hline U3 & -6.729 & 5.848 & 0.115 & 2.418 \\
\hline 708 U1 & -4.737 & 7.618 & 0.255 & 2.760 \\
\hline U2 & 4.551 & 2.404 & 0.104 & 1.551 \\
\hline 715 U1 & 12.511 & 6.562 & 0.040 & 2.562 \\
\hline U2 & 8.060 & 1.257 & 0.019 & 1.121 \\
\hline U3 & 2.580 & 9.355 & 0.594 & 3.059 \\
\hline U4 & -4.430 & 19.636 & 0.505 & 4.431 \\
\hline 716 U1 & 3.059 & 3.524 & 0.276 & 1.877 \\
\hline 721 U1 & 6.785 & 8.259 & 0.152 & 2.874 \\
\hline 722 U1 & -5.810 & 11.100 & 0.249 & 3.332 \\
\hline U2 & 4.577 & 8.061 & 0.279 & 2.839 \\
\hline 729 U1 & 2.221 & 7.029 & 0.595 & 2.651 \\
\hline U2 & -6.259 & 3.772 & 0.088 & 1.942 \\
\hline 735 U1 & 10.185 & 10.020 & 0.088 & 3.165 \\
\hline
\end{tabular}




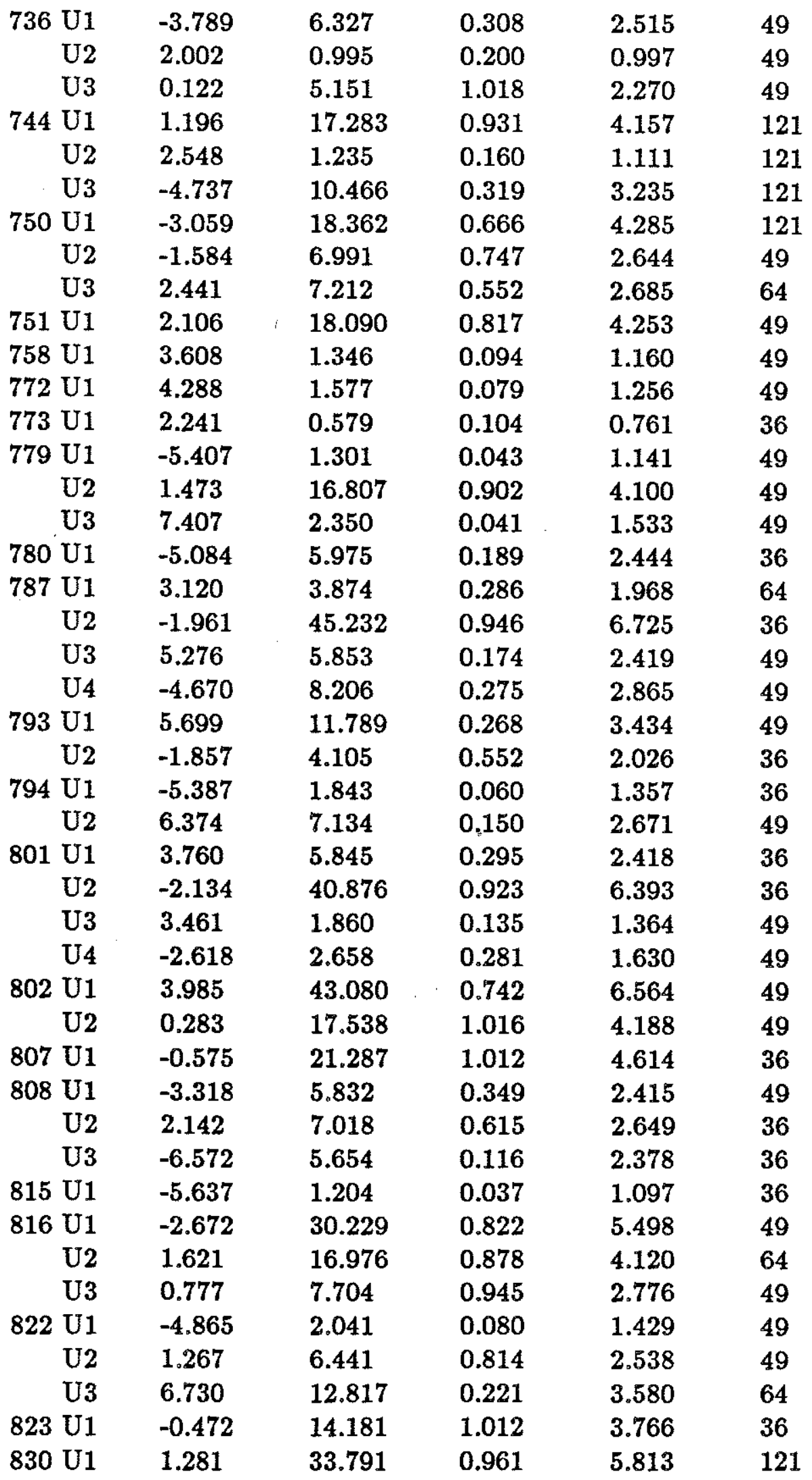




\begin{tabular}{|c|c|c|c|c|c|}
\hline U2 & 4.800 & 7.074 & 0.236 & 2.660 & 81 \\
\hline U3 & 1.204 & 2.843 & 0.669 & 1.686 & 64 \\
\hline 836 U1 & 5.337 & 9.696 & 0.256 & 3.114 & 36 \\
\hline $\mathrm{U} 2$ & 0.872 & 45.911 & 0.992 & 6.776 & 121 \\
\hline $837 \mathrm{U1}$ & -1.211 & 2.185 & 0.604 & 1.478 & 64 \\
\hline 844 U1 & 4.956 & 1.180 & 0.046 & 1.086 & 36 \\
\hline $\mathrm{U} 2$ & -2.003 & 17.167 & 0.824 & 4.143 & 49 \\
\hline 845 U1 & 2.281 & 5.439 & 0.513 & 2.332 & 121 \\
\hline $865 \mathrm{U} 1$ & -5.150 & 6.097 & 0.187 & 2.469 & 64 \\
\hline U2 & 4.101 & 4.562 & 0.214 & 2.136 & 64 \\
\hline U3 & -4.587 & 29.789 & 0.590 & 5.458 & 81 \\
\hline U4 & 0.019 & 36.865 & 1.029 & 6.072 & 36 \\
\hline 866 U1 & 3.035 & 5.254 & 0.366 & 2.292 & 49 \\
\hline 873 U1 & -4.072 & 19.614 & 0.546 & 4.429 & 81 \\
\hline U2 & 6.919 & 5.432 & 0.102 & 2.331 & 49 \\
\hline U3 & -0.940 & 14.347 & 0.949 & 3.788 & 121 \\
\hline 887 U1 & 2.564 & 7.602 & 0.544 & 2.757 & 36 \\
\hline $\mathrm{U} 2$ & -2.878 & 8.729 & 0.518 & 2.955 & 49 \\
\hline 888 U1 & -3.852 & 1.212 & 0.076 & 1.101 & 36 \\
\hline $901 \mathrm{U1}$ & -3.899 & 5.717 & 0.274 & 2.391 & 121 \\
\hline $902 \mathrm{U1}$ & -1.488 & 2.655 & 0.551 & 1.630 & 49 \\
\hline $\mathrm{U} 2$ & -3.649 & 11.407 & 0.463 & 3.377 & 121 \\
\hline $916 \mathrm{U1}$ & 2.357 & 11.493 & 0.681 & 3.390 & 64 \\
\hline U2 & -6.806 & 2.676 & 0.055 & 1.636 & 49 \\
\hline $\mathrm{U} 3$ & -3.508 & 6.299 & 0.341 & 2.510 & 49 \\
\hline 930 U1 & 0.467 & 6.682 & 0.988 & 2.585 & 49 \\
\hline $\mathrm{U} 2$ & -5.344 & 2.066 & 0.068 & 1.437 & 49 \\
\hline $951 \mathrm{U1}$ & -3.284 & 20.237 & 0.664 & 4.499 & 36 \\
\hline U2 & 1.080 & 1.115 & 0.495 & 1.056 & 36 \\
\hline U3 & 2.141 & 0.602 & 0.116 & 0.776 & 36 \\
\hline $952 \mathrm{U1}$ & -2.710 & 1.366 & 0.157 & 1.169 & 49 \\
\hline U2 & -2.246 & 10.658 & 0.688 & 3.265 & 49 \\
\hline 959 U1 & -2.170 & 0.891 & 0.160 & 0.944 & 49 \\
\hline $\mathrm{U} 2$ & -2.754 & 0.297 & 0.038 & 0.545 & 36 \\
\hline U3 & -5.150 & 4.594 & 0.148 & 2.143 & 49 \\
\hline 973 U1 & 3.063 & 4.395 & 0.322 & 2.096 & 36 \\
\hline U2 & -1.423 & 0.671 & 0.251 & 0.819 & 36 \\
\hline U3 & -6.993 & 4.408 & 0.083 & 2.100 & 49 \\
\hline 987 U1 & -5.437 & 6.194 & 0.173 & 2.489 & 121 \\
\hline 988 U1 & 6.973 & 17.335 & 0.265 & 4.164 & 36 \\
\hline U2 & -1.070 & 16.781 & 0.945 & 4.097 & 100 \\
\hline $1002 \mathrm{U} 1$ & 4.029 & 2.348 & 0.127 & 1.532 & 121 \\
\hline U2 & 5.439 & 6.236 & 0.175 & 2.497 & 49 \\
\hline U3 & -3.103 & 7.825 & 0.450 & 2.797 & 121 \\
\hline
\end{tabular}




$\begin{array}{rlllll}1003 \text { U1 } & 0.150 & 3.905 & 1.010 & 1.976 & 64 \\ 1008 \text { U1 } & 3.190 & 7.833 & 0.440 & 2.799 & 36 \\ \text { U2 } & 6.538 & 68.106 & 0.618 & 8.253 & 121 \\ 1009 \text { U1 } & -1.800 & 10.402 & 0.767 & 3.225 & 121 \\ \text { U2 } & 4.763 & 3.753 & 0.142 & 1.937 & 121 \\ 1016 \text { U1 } & 3.393 & 13.884 & 0.553 & 3.726 & 49 \\ \text { U2 } & -7.608 & 9.405 & 0.140 & 3.067 & 81 \\ 1017 \text { U1 } & 2.008 & 2.917 & 0.425 & 1.708 & 36 \\ 1030 \text { U1 } & -4.464 & 12.801 & 0.392 & 3.578 & 121 \\ 1031 \text { U1 } & 5.608 & 4.806 & 0.133 & 2.192 & 64 \\ \text { U2 } & 1.669 & 21.126 & 0.906 & 4.596 & 36 \\ \text { U3 } & -5.088 & 5.881 & 0.186 & 2.425 & 36 \\ 1051 \text { U1 } & 3.665 & 46.865 & 0.782 & 6.846 & 121 \\ 1052 \text { U1 } & 1.631 & 16.638 & 0.870 & 4.079 & 100 \\ \text { U2 } & 5.786 & 21.046 & 0.389 & 4.588 & 49 \\ 1059 \text { U1 } & 0.816 & 16.569 & 0.981 & 4.070 & 49 \\ \text { U2 } & -2.113 & 5.648 & 0.565 & 2.377 & 49 \\ 1060 \text { U1 } & -1.723 & 0.750 & 0.203 & 0.866 & 36 \\ 1074 \text { U1 } & -12.984 & 16.989 & 0.092 & 4.122 & 49 \\ \text { U2 } & 6.019 & 86.731 & 0.716 & 9.313 & 49 \\ \text { U3 } & 5.368 & 25.262 & 0.472 & 5.026 & 49 \\ \text { U4 } & -1.800 & 6.988 & 0.697 & 2.643 & 36\end{array}$

$\mathrm{V}$ component

\begin{tabular}{|c|c|c|c|c|}
\hline REV & $\frac{\text { MEAN }}{(\mathrm{m} / \mathrm{s})}$ & $\frac{\text { VAR }}{\left(\mathrm{m}^{2} / \mathrm{s}^{2}\right)}$ & WVAR & $\frac{\text { SDEV }}{(\mathrm{m} / \mathrm{s})}$ \\
\hline $658 \mathrm{~V} 1$ & 2.348 & 5.671 & 0.512 & 2.381 \\
\hline V2 & 0.783 & 0.763 & 0.563 & 0.873 \\
\hline $664 \mathrm{V1}$ & 0.697 & 7.154 & 0.961 & 2.675 \\
\hline $\mathrm{V} 2$ & 0.504 & 0.670 & 0.733 & 0.818 \\
\hline V3 & 6.018 & 9.940 & 0.217 & 3.153 \\
\hline $665 \mathrm{~V} 1$ & 6.552 & 8.178 & 0.161 & 2.860 \\
\hline $672 \mathrm{V1}$ & 1.903 & 4.385 & 0.556 & 2.094 \\
\hline V2 & 5.178 & 1.530 & 0.054 & 1.237 \\
\hline V3 & -0.345 & 2.044 & 0.971 & 1.430 \\
\hline $678 \mathrm{~V} 1$ & 2.290 & 2.559 & 0.330 & 1.600 \\
\hline $679 \mathrm{~V} 1$ & 0.300 & 1.428 & 0.966 & 1.195 \\
\hline V2 & 4.115 & 5.656 & 0.252 & 2.378 \\
\hline $686 \mathrm{~V} 1$ & -1.647 & 6.412 & 0.713 & 2.532 \\
\hline V2 & -0.190 & 4.279 & 1.012 & 2.069 \\
\hline $692 \mathrm{~V} 1$ & 4.238 & 2.136 & 0.107 & 1.462 \\
\hline $693 \mathrm{~V} 1$ & -0.022 & 8.535 & 1.021 & 2.921 \\
\hline
\end{tabular}




\begin{tabular}{|c|c|c|c|c|c|}
\hline V2 & 2.856 & 0.576 & 0.066 & 0.759 & 36 \\
\hline V3 & 5.321 & 1.389 & 0.047 & 1.179 & 36 \\
\hline $700 \mathrm{~V} 1$ & -5.105 & 1.591 & 0.058 & 1.261 & 100 \\
\hline $701 \mathrm{~V} 1$ & -2.044 & 1.692 & 0.291 & 1.301 & 36 \\
\hline V2 & 3.107 & 1.875 & 0.163 & 1.369 & 36 \\
\hline V3 & 0.644 & 1.992 & 0.847 & 1.412 & 36 \\
\hline $708 \mathrm{~V} 1$ & 1.106 & 3.433 & 0.753 & 1.853 & 36 \\
\hline V2 & 0.611 & 1.833 & 0.850 & 1.354 & 36 \\
\hline 715 V1 & -2.938 & 33.891 & 0.810 & 5.822 & 49 \\
\hline V2 & -0.544 & 4.302 & 0.954 & 2.074 & 49 \\
\hline V3 & -0.121 & 0.544 & 1.001 & 0.737 & 36 \\
\hline V4 & -1.537 & 10.174 & 0.825 & 3.190 & 49 \\
\hline $716 \mathrm{~V} 1$ & 2.665 & 1.623 & 0.187 & 1.274 & 36 \\
\hline $721 \mathrm{~V} 1$ & -1.090 & 6.569 & 0.858 & 2.563 & 64 \\
\hline $722 \mathrm{~V} 1$ & 0.365 & 4.426 & 0.998 & 2.104 & 36 \\
\hline V2 & -2.375 & 5.131 & 0.481 & 2.265 & 49 \\
\hline $729 \mathrm{~V} 1$ & -1.376 & 1.944 & 0.512 & 1.394 & 49 \\
\hline V2 & -0.686 & 3.524 & 0.904 & 1.877 & 36 \\
\hline $735 \mathrm{~V} 1$ & -0.944 & 3.958 & 0.835 & 1.990 & 36 \\
\hline $736 \mathrm{V1}$ & -0.725 & 2.596 & 0.846 & 1.611 & 49 \\
\hline V2 & -3.226 & 1.533 & 0.129 & 1.238 & 49 \\
\hline V3 & 4.880 & 0.395 & 0.016 & 0.628 & 49 \\
\hline $744 \mathrm{~V} 1$ & 0.242 & 7.246 & 1.000 & 2.692 & 121 \\
\hline V2 & 3.527 & 4.739 & 0.276 & 2.177 & 121 \\
\hline V3 & 1.789 & 2.227 & 0.412 & 1.492 & 121 \\
\hline $750 \mathrm{~V} 1$ & -0.649 & 2.233 & 0.847 & 1.494 & 121 \\
\hline $\mathrm{V} 2$ & -0.735 & 3.176 & 0.870 & 1.782 & 49 \\
\hline V3 & 1.711 & 9.999 & 0.783 & 3.162 & 64 \\
\hline $751 \mathrm{~V} 1$ & 3.950 & 5.721 & 0.270 & 2.392 & 49 \\
\hline $758 \mathrm{~V} 1$ & 3.703 & 3.839 & 0.220 & 1.959 & 49 \\
\hline $772 \mathrm{V1}$ & 3.010 & 5.429 & 0.378 & 2.330 & 49 \\
\hline $773 \mathrm{~V} 1$ & -0.524 & 2.342 & 0.918 & 1.530 & 36 \\
\hline $779 \mathrm{~V} 1$ & -0.439 & 1.081 & 0.864 & 1.040 & 49 \\
\hline V2 & 3.342 & 2.503 & 0.184 & 1.582 & 49 \\
\hline V3 & 0.307 & 35.855 & 1.018 & 5.988 & 49 \\
\hline $780 \mathrm{~V} 1$ & 2.052 & 6.889 & 0.632 & 2.625 & 36 \\
\hline $787 \mathrm{~V} 1$ & -4.738 & 28.877 & 0.568 & 5.374 & 64 \\
\hline V2 & 4.819 & 7.055 & 0.235 & 2.656 & 36 \\
\hline V3 & 2.837 & 8.013 & 0.504 & 2.831 & 49 \\
\hline V4 & 1.296 & 5.452 & 0.777 & 2.335 & 49 \\
\hline $793 \mathrm{V1}$ & -5.169 & 1.560 & 0.055 & 1.249 & 49 \\
\hline $\mathrm{V} 2$ & -6.090 & 11.234 & 0.234 & 3.352 & 36 \\
\hline $794 \mathrm{~V} 1$ & 2.540 & 6.962 & 0.527 & 2.639 & 36 \\
\hline V2 & 0.223 & 2.434 & 1.000 & 1.560 & 49 \\
\hline
\end{tabular}




\begin{tabular}{|c|c|c|c|c|c|}
\hline $801 \mathrm{V1}$ & -6.323 & 6.528 & 0.141 & 2.555 & 36 \\
\hline $\mathrm{V} 2$ & 2.917 & 10.017 & 0.549 & 3.165 & 36 \\
\hline V3 & -0.310 & 8.872 & 1.010 & 2.979 & 49 \\
\hline V4 & -1.391 & 3.634 & 0.661 & 1.906 & 49 \\
\hline 802 V1 & -2.567 & 19.814 & 0.762 & 4.451 & 49 \\
\hline $\mathrm{V} 2$ & 1.444 & 3.897 & 0.660 & 1.974 & 49 \\
\hline $807 \mathrm{~V} 1$ & 0.178 & 6.640 & 1.024 & 2.577 & 36 \\
\hline $808 \mathrm{~V} 1$ & 1.189 & 9.143 & 0.882 & 3.024 & 49 \\
\hline $\mathrm{V} 2$ & 2.250 & 2.495 & 0.333 & 1.579 & 36 \\
\hline V3 & -6.572 & 5.654 & 0.116 & 2.378 & 36 \\
\hline $815 \mathrm{~V} 1$ & -0.674 & 3.190 & 0.897 & 1.786 & 36 \\
\hline $816 \mathrm{~V} 1$ & -1.467 & 19.705 & 0.918 & 4.439 & 49 \\
\hline $\mathrm{V} 2$ & -2.284 & 8.930 & 0.637 & 2.988 & 64 \\
\hline V3 & 3.606 & 4.746 & 0.269 & 2.178 & 49 \\
\hline 822 V1 & 0.344 & 1.206 & 0.928 & 1.098 & 49 \\
\hline V2 & 1.321 & 1.504 & 0.467 & 1.226 & 49 \\
\hline V3 & 2.547 & 25.010 & 0.804 & 5.001 & 64 \\
\hline $823 \mathrm{~V} 1$ & -2.613 & 14.472 & 0.693 & 3.804 & 36 \\
\hline $830 \mathrm{~V} 1$ & 2.843 & 14.832 & 0.651 & 3.851 & 121 \\
\hline V2 & 3.422 & 5.288 & 0.312 & 2.299 & 81 \\
\hline V3 & 3.180 & 2.167 & 0.177 & 1.472 & 64 \\
\hline $836 \mathrm{~V} 1$ & 0.768 & 3.045 & 0.858 & 1.745 & 36 \\
\hline $\mathrm{V} 2$ & 5.857 & 13.292 & 0.280 & 3.646 & 121 \\
\hline $837 \mathrm{~V} 1$ & 1.457 & 7.338 & 0.785 & 2.709 & 64 \\
\hline 844 V1 & -1.584 & 0.739 & 0.229 & 0.859 & 36 \\
\hline V2 & -0.616 & 5.484 & 0.953 & 2.342 & 49 \\
\hline $845 \mathrm{~V} 1$ & -2.898 & 2.840 & 0.253 & 1.685 & 121 \\
\hline $865 \mathrm{~V} 1$ & 2.096 & 9.725 & 0.696 & 3.119 & 64 \\
\hline V2 & 3.336 & 7.210 & 0.396 & 2.685 & 64 \\
\hline V3 & 4.180 & 17.918 & 0.509 & 4.233 & 81 \\
\hline V4 & -0.401 & 45.699 & 1.025 & 6.760 & 36 \\
\hline 866 V1 & -1.525 & 8.263 & 0.793 & 2.874 & 49 \\
\hline 873 V1 & 5.177 & 13.500 & 0.336 & 3.674 & 81 \\
\hline $\mathrm{V} 2$ & 5.209 & 6.631 & 0.197 & 2.575 & 49 \\
\hline V3 & 3.961 & 2.557 & 0.140 & 1.599 & 121 \\
\hline $887 \mathrm{~V} 1$ & 3.655 & 6.857 & 0.342 & 2.618 & 36 \\
\hline V2 & 1.205 & 4.756 & 0.778 & 2.181 & 49 \\
\hline $888 \mathrm{VI}$ & 0.584 & 0.253 & 0.432 & 0.503 & 36 \\
\hline $901 \mathrm{V1}$ & 1.364 & 1.604 & 0.465 & 1.267 & 121 \\
\hline $902 \mathrm{~V} 1$ & 5.982 & 22.001 & 0.384 & 4.691 & 49 \\
\hline $\mathrm{V} 2$ & -0.133 & 2.233 & 1.000 & 1.494 & 121 \\
\hline $916 \mathrm{V1}$ & -8.635 & 4.288 & 0.054 & 2.071 & 64 \\
\hline V2 & -1.126 & 5.284 & 0.820 & 2.299 & 49 \\
\hline V3 & 0.894 & 4.652 & 0.869 & 2.157 & 49 \\
\hline
\end{tabular}




$\begin{array}{rlllll}930 \text { V1 } & -3.137 & 9.054 & 0.484 & 3.009 & 49 \\ \text { V2 } & -0.654 & 3.297 & 0.901 & 1.816 & 49 \\ 951 \text { V1 } & -2.505 & 2.700 & 0.303 & 1.643 & 36 \\ \text { V2 } & -2.831 & 0.990 & 0.110 & 0.995 & 36 \\ \text { V3 } & -2.401 & 2.285 & 0.286 & 1.512 & 36 \\ 952 \text { V1 } & 6.055 & 3.046 & 0.077 & 1.745 & 49 \\ \text { V2 } & 5.842 & 17.202 & 0.337 & 4.148 & 49 \\ 959 \text { V1 } & 0.719 & 9.274 & 0.966 & 3.045 & 49 \\ \text { V2 } & 0.540 & 0.053 & 0.155 & 0.231 & 36 \\ \text { V3 } & -4.432 & 5.942 & 0.233 & 2.438 & 49 \\ 973 \text { V1 } & -2.453 & 1.896 & 0.241 & 1.377 & 36 \\ \text { V2 } & -3.119 & 0.834 & 0.079 & 0.913 & 36 \\ \text { V3 } & -0.746 & 2.177 & 0.810 & 1.475 & 49 \\ 987 \text { V1 } & -3.004 & 3.044 & 0.253 & 1.745 & 121 \\ 988 \text { V1 } & 10.503 & 32.575 & 0.229 & 5.707 & 36 \\ \text { V2 } & 3.623 & 2.465 & 0.158 & 1.570 & 100 \\ 1002 \text { V1 } & 9.666 & 2.279 & 0.024 & 1.510 & 121 \\ \text { V2 } & 6.420 & 3.225 & 0.073 & 1.796 & 49 \\ \text { V3 } & 1.454 & 2.334 & 0.527 & 1.528 & 121 \\ 1003 \text { V1 } & -4.668 & 5.978 & 0.216 & 2.445 & 64 \\ 1008 \text { V1 } & 6.507 & 4.642 & 0.099 & 2.155 & 36 \\ \text { V2 } & 12.315 & 28.861 & 0.160 & 5.372 & 121 \\ 1009 \text { V1 } & 2.683 & 1.841 & 0.204 & 1.357 & 121 \\ \text { V2 } & -0.950 & 5.578 & 0.867 & 2.362 & 121 \\ 1016 \text { V1 } & 5.737 & 7.319 & 0.183 & 2.705 & 49 \\ \text { V2 } & 0.389 & 22.008 & 1.005 & 4.691 & 81 \\ 1017 \text { V1 } & 3.661 & 8.947 & 0.405 & 2.991 & 36 \\ 1030 \text { V1 } & -2.345 & 6.308 & 0.537 & 2.512 & 121 \\ 1031 \text { V1 } & 3.049 & 7.606 & 0.453 & 2.758 & 64 \\ \text { V2 } & 3.351 & 9.056 & 0.452 & 3.009 & 36 \\ \text { V3 } & -5.074 & 2.710 & 0.095 & 1.646 & 36 \\ 1060 \text { V1 } & -1.559 & 1.211 & 0.336 & 1.100 & 36 \\ 1074 \text { V1 } & 4.286 & 55.585 & 0.763 & 7.456 & 49 \\ \text { V2 } & -1.321 & 140.918 & 1.008 & 11.871 & 49 \\ \text { V3 } & 0.160 & 18.131 & 1.019 & 4.258 & 49 \\ \text { V4 } & -1.124 & 2.801 & 0.702 & 1.674 & 36\end{array}$


Table 4: Mapped Atlas SASS component wind statistics - 1-day averages

\begin{tabular}{|c|c|c|c|c|c|}
\hline DAY & $\frac{\text { MEAN }}{(\mathrm{m} / \mathrm{s})}$ & $\frac{\text { VAR }}{\left(\mathrm{m}^{2} / \mathrm{s}^{2}\right)}$ & WVAR & $\frac{\text { SDEV }}{(\mathrm{m} / \mathrm{s})}$ & $\underline{N}$ \\
\hline $224 \mathrm{U}$ & -0.109 & 27.102 & 1.002 & 5.206 & 378 \\
\hline V & 2.548 & 9.800 & 0.603 & 3.130 & 378 \\
\hline $225 \mathrm{U}$ & -2.146 & 26.860 & 0.856 & 5.183 & 340 \\
\hline V & 1.444 & 8.633 & 0.807 & 2.938 & 340 \\
\hline $226 \mathrm{U}$ & -2.580 & 26.378 & 0.800 & 5.136 & 463 \\
\hline V & -0.201 & 14.313 & 0.999 & 3.783 & 463 \\
\hline $227 \mathrm{U}$ & 1.222 & 41.364 & 0.967 & 6.432 & 499 \\
\hline V & -1.085 & 12.714 & 0.917 & 3.566 & 499 \\
\hline $228 \mathrm{U}$ & 2.929 & 41.766 & 0.831 & 6.463 & 453 \\
\hline V & -0.926 & 9.551 & 0.919 & 3.090 & 453 \\
\hline $229 \mathrm{U}$ & 0.077 & 23.575 & 1.001 & 4.855 & 631 \\
\hline V & 0.938 & 8.676 & 0.909 & 2.946 & 631 \\
\hline $230 \mathrm{U}$ & -0.189 & 19.515 & 1.000 & 4.418 & 695 \\
\hline V & 1.500 & 7.567 & 0.772 & 2.751 & 695 \\
\hline $231 \mathrm{U}$ & 3.490 & 1.869 & 0.133 & 1.367 & 134 \\
\hline V & 2.314 & 7.031 & 0.570 & 2.652 & 134 \\
\hline $232 \mathrm{U}$ & 0.937 & 27.555 & 0.971 & 5.249 & 466 \\
\hline V & 0.928 & 18.717 & 0.958 & 4.326 & 466 \\
\hline $233 \mathrm{U}$ & 1.256 & 27.875 & 0.948 & 5.280 & 636 \\
\hline V & -0.878 & 20.890 & 0.966 & 4.571 & 636 \\
\hline $234 \mathrm{U}$ & -0.069 & 22.367 & 1.001 & 4.729 & 623 \\
\hline $\mathrm{V}$ & -0.661 & 16.108 & 0.975 & 4.013 & 623 \\
\hline $235 \mathrm{U}$ & 0.930 & 25.584 & 0.969 & 5.058 & 662 \\
\hline V & 1.383 & 14.450 & 0.884 & 3.801 & 662 \\
\hline $236 \mathrm{U}$ & 1.728 & 22.131 & 0.882 & 4.704 & 693 \\
\hline V & 1.755 & 15.901 & 0.839 & 3.988 & 693 \\
\hline $237 \mathrm{U}$ & 1.730 & 12.742 & 0.813 & 3.570 & 206 \\
\hline V & -2.126 & 4.021 & 0.472 & 2.005 & 206 \\
\hline $238 \mathrm{U}$ & -0.722 & 30.494 & 0.985 & 5.522 & 545 \\
\hline V & 3.213 & 15.631 & 0.603 & 3.954 & 545 \\
\hline $239 \mathrm{U}$ & -0.785 & 24.319 & 0.978 & 4.931 & 372 \\
\hline V & 3.671 & 8.444 & 0.386 & 2.906 & 372 \\
\hline $240 \mathrm{U}$ & -2.849 & 10.468 & 0.564 & 3.235 & 412 \\
\hline V & 1.587 & 8.519 & 0.773 & 2.919 & 412 \\
\hline $241 \mathrm{U}$ & -2.960 & 13.620 & 0.609 & 3.690 & 453 \\
\hline V & -0.269 & 20.098 & 0.999 & 4.483 & 453 \\
\hline $242 \mathrm{U}$ & -2.283 & 18.757 & 0.785 & 4.331 & 260 \\
\hline V & -2.884 & 17.582 & 0.681 & 4.193 & 260 \\
\hline
\end{tabular}




\begin{tabular}{|c|c|c|c|c|}
\hline $243 \mathrm{U}$ & -2.438 & 12.859 & 0.689 & 3.586 \\
\hline V & -1.896 & 7.668 & 0.686 & 2.769 \\
\hline $244 \mathrm{U}$ & -2.067 & 9.356 & 0.688 & 3.059 \\
\hline V & 0.417 & 20.773 & 0.995 & 4.558 \\
\hline $245 \mathrm{U}$ & -2.908 & 12.271 & 0.593 & 3.503 \\
\hline . V & -1.567 & 7.474 & 0.755 & 2.734 \\
\hline $246 \mathrm{U}$ & -2.110 & 26.434 & 0.858 & 5.141 \\
\hline V & 0.370 & 23.519 & 0.997 & 4.850 \\
\hline $247 \mathrm{U}$ & -0.205 & 24.179 & 1.000 & 4.917 \\
\hline $\mathrm{V}$ & 2.840 & 31.501 & 0.797 & 5.613 \\
\hline $248 \mathrm{U}$ & 1.386 & 32.991 & 0.946 & 5.744 \\
\hline V & 4.065 & 33.472 & 0.670 & 5.786 \\
\hline $249 \mathrm{U}$ & -1.612 & 33.314 & 0.930 & 5.772 \\
\hline V & 0.694 & 20.302 & 0.979 & 4.5067 \\
\hline $250 \mathrm{U}$ & -1.184 & 30.780 & 0.960 & 5.548 \\
\hline V & -0.586 & 16.216 & 0.983 & 4.027 \\
\hline $251 \mathrm{U}$ & 1.892 & 29.322 & 0.893 & 5.415 \\
\hline V & 2.939 & 48.639 & 0.851 & 6.974 \\
\hline $252 \mathrm{U}$ & -0.847 & 60.832 & 0.991 & 7.799 \\
\hline $\mathrm{V}$ & 2.138 & 47.377 & 0.915 & 6.883 \\
\hline
\end{tabular}


Table 5: Mapped Atlas SASS component wind statistics 1-day/10 deg latitude averages

$20-30 \mathrm{~N}$

\begin{tabular}{|c|c|c|c|c|c|c|}
\hline DAY & & $\begin{array}{l}\text { MEAN } \\
(\mathrm{m} / \mathrm{s})\end{array}$ & $\begin{array}{c}\mathrm{VAR} \\
\left(\mathrm{m}^{\wedge} 2 / \mathrm{s}^{\wedge} 2\right)\end{array}$ & WGTVAR & $\begin{array}{l}\text { SDEV } \\
(\mathrm{m} / \mathrm{s})\end{array}$ & $N$ \\
\hline 224 & $\mathrm{U}$ & -5.065 & 2.995 & 0.105 & 1.731 & 172 \\
\hline & V & 0.425 & 2.455 & 0.936 & 1.567 & 172 \\
\hline 225 & $\mathrm{U}$ & -5.751 & 12.935 & 0.282 & 3.597 & 157 \\
\hline & V & 0.874 & 6.562 & 0.901 & 2.562 & 157 \\
\hline 226 & $\mathrm{U}$ & -6.214 & 6.767 & 0.149 & 2.601 & 234 \\
\hline & V & -2.127 & 10.329 & 0.698 & 3.214 & 234 \\
\hline 227 & $\mathrm{U}$ & -3.709 & 15.100 & 0.524 & 3.886 & 257 \\
\hline & $\mathrm{V}$ & -2.051 & 9.979 & 0.705 & 3.159 & 257 \\
\hline 228 & $\mathrm{U}$ & -0.885 & 29.174 & 0.978 & 5.401 & 255 \\
\hline & $\mathrm{V}$ & -1.079 & 5.259 & 0.821 & 2.293 & 255 \\
\hline 229 & $\mathrm{U}$ & -3.432 & 16.122 & 0.579 & 4.015 & 255 \\
\hline & $\mathrm{V}$ & 0.348 & 4.332 & 0.977 & 2.081 & 255 \\
\hline 230 & $\mathrm{U}$ & -2.887 & 20.574 & 0.713 & 4.536 & 291 \\
\hline & V & 1.139 & 5.638 & 0.815 & 2.375 & 291 \\
\hline 231 & $\mathrm{U}$ & none & & & & \\
\hline & V & none & & & & \\
\hline 232 & $\mathrm{U}$ & -5.050 & 5.105 & 0.167 & 2.259 & 134 \\
\hline & $\mathrm{V}$ & 0.864 & 5.246 & 0.881 & 2.290 & 134 \\
\hline 233 & $\mathrm{U}$ & -2.935 & 12.661 & 0.597 & 3.558 & 183 \\
\hline & $\mathrm{V}$ & 0.861 & 6.828 & 0.907 & 2.613 & 183 \\
\hline 234 & $\mathrm{U}$ & -1.550 & 13.212 & 0.849 & 3.635 & 268 \\
\hline & V & 1.120 & 7.463 & 0.859 & 2.732 & 268 \\
\hline 235 & $U$ & -1.683 & 12.889 & 0.823 & 3.590 & 234 \\
\hline & $\mathrm{V}$ & 1.216 & 9.640 & 0.870 & 3.105 & 234 \\
\hline 236 & $\mathrm{U}$ & 0.595 & 9.093 & 0.966 & 3.015 & 298 \\
\hline & $\mathrm{V}$ & -0.282 & 10.091 & 0.995 & 3.177 & 298 \\
\hline 237 & $\mathrm{U}$ & 1.047 & 12.525 & 0.925 & 3.539 & 170 \\
\hline & V & -2.240 & 4.649 & 0.482 & 2.156 & 170 \\
\hline 238 & $\mathrm{U}$ & -1.259 & 18.209 & 0.924 & 4.267 & 234 \\
\hline & $\mathrm{V}$ & 2.302 & 10.170 & 0.659 & 3.189 & 234 \\
\hline 239 & $\mathrm{U}$ & -1.910 & 12.091 & 0.771 & 3.477 & 206 \\
\hline & $\mathrm{V}$ & 2.715 & 4.913 & 0.401 & 2.216 & 206 \\
\hline 240 & $\mathrm{U}$ & -3.649 & 7.836 & 0.371 & 2.799 & 327 \\
\hline & V & 0.700 & 2.601 & 0.844 & 1.613 & 327 \\
\hline 241 & $\mathrm{U}$ & -3.729 & 8.151 & 0.370 & 2.855 & 291 \\
\hline & $\mathrm{V}$ & 0.662 & 2.836 & 0.869 & 1.684 & 291 \\
\hline 242 & $\mathrm{U}$ & -4.426 & 4.991 & 0.203 & 2.234 & 98 \\
\hline & V & 0.120 & 4.539 & 1.007 & 2.131 & 98 \\
\hline 243 & $\mathrm{U}$ & -5.344 & 2.066 & 0.068 & 1.437 & 49 \\
\hline & V & -3.137 & 9.054 & 0.484 & 3.009 & 49 \\
\hline 244 & $\mathrm{U}$ & -3.756 & 8.655 & 0.381 & 2.942 & 134 \\
\hline & V & -0.079 & 26.388 & 1.007 & 5.137 & 134 \\
\hline 245 & $U$ & -4.822 & 8.329 & 0.264 & 2.886 & 134 \\
\hline & V & -2.731 & 5.708 & 0.435 & 2.389 & 134 \\
\hline 246 & $\mathrm{U}$ & -3.176 & 14.065 & 0.584 & 3.750 & 257 \\
\hline & V & -0.441 & 13.058 & 0.989 & 3.614 & 257 \\
\hline 247 & U & -3.334 & 12.896 & 0.538 & 3.591 & 342 \\
\hline & $\mathrm{V}$ & 0.511 & 10.147 & 0.978 & 3.185 & 342 \\
\hline & $\mathrm{U}$ & -3.168 & 16.285 & 0.620 & 4.035 & 359 \\
\hline & V & 1.849 & 8.293 & 0.709 & 2.880 & 359 \\
\hline
\end{tabular}




$\begin{array}{rrrrrrr}249 & \mathrm{U} & -3.894 & 22.368 & 0.597 & 4.729 & 310 \\ & \mathrm{~V} & -0.589 & 18.208 & 0.984 & 4.267 & 310 \\ 250 & \mathrm{U} & -3.436 & 18.987 & 0.619 & 4.357 & 193 \\ & \mathrm{~V} & -1.792 & 13.260 & 0.808 & 3.641 & 193 \\ 251 & \mathrm{U} & -0.013 & 13.783 & 1.005 & 3.713 & 185 \\ & \mathrm{~V} & 2.239 & 17.110 & 0.777 & 4.136 & 185 \\ 252 & \mathrm{U} & -1.904 & 4.547 & 0.559 & 2.132 & 121 \\ & \mathrm{~V} & 1.404 & 15.760 & 0.895 & 3.970 & 121\end{array}$

$30-40 \mathrm{~N}$

\begin{tabular}{|c|c|c|c|c|c|}
\hline DAY & $\begin{array}{l}\text { MEAN } \\
(\mathrm{m} / \mathrm{s})\end{array}$ & $\begin{array}{c}\text { VAR } \\
\left(m^{\wedge} 2 / s^{\wedge} 2\right)\end{array}$ & WGTVAR & $\begin{array}{l}\text { SDEV } \\
(\mathrm{m} / \mathrm{s})\end{array}$ & $\mathbf{N}$ \\
\hline 224 & 3.592 & 9.611 & 0.428 & 3.100 & 170 \\
\hline & 4.831 & 8.551 & 0.269 & 2.924 & 170 \\
\hline 225 & -0.527 & 15.173 & 0.987 & 3.895 & 183 \\
\hline & 2.368 & 10.930 & 0.663 & 3.306 & 183 \\
\hline 226 & 1.859 & 18.166 & 0.844 & 4.262 & 193 \\
\hline & 1.305 & 11.022 & 0.870 & 3.320 & 193 \\
\hline 227 & 4.921 & 6.697 & 0.217 & 2.588 & 193 \\
\hline & 0.671 & 5.973 & 0.934 & 2.444 & 193 \\
\hline 228 & 5.876 & 8.862 & 0.204 & 2.977 & 198 \\
\hline & -0.590 & 7.498 & 0.960 & 2.738 & 198 \\
\hline 229 & 1.883 & 2.979 & 0.457 & 1.726 & 219 \\
\hline & 2.319 & 12.228 & 0.697 & 3.497 & 219 \\
\hline 230 & 1.525 & 9.032 & 0.798 & 3.005 & 219 \\
\hline & 2.668 & 7.950 & 0.529 & 2.820 & 219 \\
\hline 231 & 3.421 & 2.178 & 0.157 & 1.476 & 85 \\
\hline & 1.513 & 7.164 & 0.765 & 2.677 & 85 \\
\hline 232 & 3.410 & 19.260 & 0.625 & 4.389 & 268 \\
\hline & 2.313 & 13.548 & 0.719 & 3.681 & 268 \\
\hline 233 & 3.408 & 27.240 & 0.703 & 5.219 & 353 \\
\hline & -0.548 & 20.704 & 0.988 & 4.550 & 353 \\
\hline 234 & 1.940 & 22.936 & 0.862 & 4.789 & 270 \\
\hline & -2.394 & 19.074 & 0.771 & 4.367 & 270 \\
\hline 235 & 3.294 & 17.333 & 0.616 & 4.163 & 294 \\
\hline & 0.900 & 16.828 & 0.957 & 4.102 & 294 \\
\hline 236 & 2.834 & 10.451 & 0.566 & 3.233 & 338 \\
\hline & -0.028 & 10.595 & 1.003 & 3.255 & 338 \\
\hline 237 & 2.895 & 5.721 & 0.407 & 2.392 & 157 \\
\hline & -2.597 & 2.658 & 0.283 & 1.630 & 157 \\
\hline 238 & 1.360 & 34.300 & 0.952 & 5.857 & 279 \\
\hline & 2.574 & 21.331 & 0.765 & 4.619 & 279 \\
\hline 239 & 5.075 & 10.959 & 0.300 & 3.310 & 85 \\
\hline & 4.551 & 7.243 & 0.260 & 2.691 & 85 \\
\hline & 0.228 & 8.741 & 1.006 & 2.956 & 85 \\
\hline & 4.997 & 16.767 & 0.404 & 4.095 & 85 \\
\hline & -4.147 & 9.782 & 0.364 & 3.128 & 98 \\
\hline & 2.428 & 26.265 & 0.824 & 5.125 & 98 \\
\hline & -3.169 & 17.994 & 0.646 & 4.242 & 98 \\
\hline & -2.132 & 8.116 & 0.645 & 2.849 & 98 \\
\hline & 0.467 & 6.682 & 0.988 & 2.585 & 49 \\
\hline & -3.137 & 9.054 & 0.484 & 3.009 & 49 \\
\hline & -1.407 & 7.363 & 0.793 & 2.714 & 121 \\
\hline & 1.684 & 20.751 & 0.886 & 4.555 & 121 \\
\hline & 0.154 & 10.890 & 1.012 & 3.300 & 72 \\
\hline
\end{tabular}




$\begin{array}{rrrrrrr} & \mathrm{V} & -0.957 & 3.232 & 0.788 & 1.798 & 72 \\ 246 & \mathrm{U} & 5.018 & 14.588 & 0.369 & 3.819 & 72 \\ & \mathrm{~V} & 4.025 & 59.545 & 0.795 & 7.717 & 342 \\ 247 & \mathrm{U} & 3.538 & 16.815 & 0.576 & 4.101 & 149 \\ & \mathrm{~V} & 2.643 & 54.157 & 0.891 & 7.359 & 149 \\ 248 & \mathrm{U} & 3.397 & 9.080 & 0.441 & 3.013 & 355 \\ & \mathrm{~V} & 1.544 & 23.513 & 0.910 & 4.849 & 355 \\ 249 & \mathrm{U} & 4.010 & 9.444 & 0.371 & 3.073 & 149 \\ & \mathrm{~V} & 4.081 & 9.139 & 0.355 & 3.023 & 149 \\ 250 & \mathrm{U} & 5.608 & 4.806 & 0.133 & 2.192 & 64 \\ & \mathrm{~V} & 3.049 & 7.606 & 0.453 & 2.758 & 64 \\ 251 & \mathrm{U} & 3.301 & 24.853 & 0.700 & 4.985 & 98 \\ & \mathrm{~V} & 0.160 & 61.431 & 1.010 & 7.838 & 98 \\ 252 & \mathrm{U} & 4.067 & 47.661 & 0.746 & 6.904 & 147 \\ & \mathrm{~V} & 2.026 & 69.308 & 0.950 & 8.325 & 147\end{array}$

$40-50 \mathrm{~N}$

\begin{tabular}{|c|c|c|c|c|c|c|}
\hline DAY & & $\begin{array}{c}\mathrm{MEAN} \\
(\mathrm{m} / \mathrm{s})\end{array}$ & $\begin{array}{c}\text { VAR } \\
\left(m^{\wedge} 2 / s^{\wedge} 2\right)\end{array}$ & WGTVAR & $\begin{array}{l}\text { SDEV } \\
(\mathrm{m} / \mathrm{s})\end{array}$ & $\mathrm{N}$ \\
\hline 224 & $\mathrm{U}$ & 6.097 & 4.069 & 0.099 & 2.017 & 36 \\
\hline & $\mathrm{V}$ & 1.903 & 4.385 & 0.556 & 2.094 & 36 \\
\hline 225 & U & none & & & & \\
\hline & V & none & & & & \\
\hline 226 & U & -2.764 & 6.509 & 0.466 & 2.551 & 36 \\
\hline & $\mathrm{V}$ & 4.238 & 2.136 & 0.107 & 1.462 & 36 \\
\hline 227 & $U$ & 12.511 & 6.562 & 0.040 & 2.562 & 49 \\
\hline & $\mathrm{V}$ & -2.938 & 33.891 & 0.810 & 5.822 & 49 \\
\hline 228 & $\mathrm{U}$ & 12.511 & 6.562 & 0.040 & 2.562 & 49 \\
\hline & $\mathrm{v}$ & -2.938 & 33.891 & 0.810 & 5.822 & 49 \\
\hline 229 & $\mathrm{U}$ & 3.257 & 29.912 & 0.742 & 5.469 & 157 \\
\hline & $\mathrm{v}$ & -0.030 & 6.712 & 1.006 & 2.591 & 157 \\
\hline 230 & U & 2.041 & 12.060 & 0.746 & 3.473 & 234 \\
\hline & $\mathrm{V}$ & 1.369 & 9.063 & 0.832 & 3.011 & 234 \\
\hline 231 & U & 3.608 & 1.346 & 0.094 & 1.160 & 49 \\
\hline & $\mathrm{V}$ & 3.703 & 3.839 & 0.220 & 1.959 & 49 \\
\hline 232 & $\mathrm{U}$ & 1.291 & 24.465 & 0.945 & 4.946 & 100 \\
\hline & $\mathrm{V}$ & -1.298 & 42.126 & 0.971 & 6.490 & 100 \\
\hline 233 & $\mathrm{U}$ & -0.085 & 26.023 & 1.006 & 5.101 & 172 \\
\hline & $\mathrm{V}$ & -1.418 & 37.298 & 0.954 & 6.107 & 172 \\
\hline 234 & $\mathrm{U}$ & -1.888 & 31.009 & 0.904 & 5.569 & 121 \\
\hline & $\mathrm{v}$ & 0.327 & 16.074 & 1.002 & 4.009 & 121 \\
\hline 235 & $\mathrm{U}$ & 1.943 & 38.096 & 0.913 & 6.172 & 234 \\
\hline & $\mathrm{v}$ & 1.859 & 21.419 & 0.864 & 4.628 & 234 \\
\hline 236 & $\mathrm{U}$ & 1.077 & 39.727 & 0.976 & 6.303 & 242 \\
\hline & $\mathrm{v}$ & 4.350 & 16.284 & 0.463 & 4.035 & 242 \\
\hline 237 & $U$ & none & & & & \\
\hline & v & none & & & & \\
\hline 238 & $\mathrm{U}$ & -4.329 & 24.614 & 0.570 & 4.961 & 162 \\
\hline & $\mathrm{v}$ & 4.678 & 15.861 & 0.421 & 3.983 & 162 \\
\hline 239 & U & -4.072 & 19.614 & 0.546 & 4.429 & 81 \\
\hline & $\mathrm{v}$ & 5.177 & 13.500 & 0.336 & 3.674 & 81 \\
\hline 240 & $\mathrm{U}$ & none & & & & \\
\hline & $\mathrm{v}$ & none & & & & \\
\hline 241 & U & -1.617 & 28.415 & 0.923 & 5.331 & 113 \\
\hline & $\mathrm{V}$ & -5.379 & 18.646 & 0.393 & 4.318 & 113 \\
\hline
\end{tabular}




$\begin{array}{rrrrrrr}242 & \mathrm{U} & -1.617 & 28.415 & 0.923 & 5.331 & 113 \\ & \mathrm{~V} & -5.379 & 18.646 & 0.393 & 4.318 & 113 \\ 243 & \mathrm{U} & \text { none } & & & & \\ & \mathrm{V} & \text { none } & & & & \\ 244 & \mathrm{U} & -0.344 & 5.351 & 0.990 & 2.313 & 85 \\ & \mathrm{~V} & -0.602 & 8.657 & 0.971 & 2.942 & 85 \\ 245 & \mathrm{U} & -2.170 & 0.891 & 0.160 & 0.944 & 49 \\ & \mathrm{~V} & 0.719 & 9.274 & 0.966 & 3.045 & 49 \\ 246 & \mathrm{U} & \text { none } & & & & \\ & \mathrm{V} & \text { none } & & & & \\ 247 & \mathrm{U} & 4.029 & 2.348 & 0.127 & 1.532 & 121 \\ & \mathrm{~V} & 9.666 & 2.279 & 0.024 & 1.510 & 121 \\ 248 & \mathrm{U} & 5.284 & 36.662 & 0.569 & 6.055 & 242 \\ & \mathrm{~V} & 10.991 & 17.267 & 0.125 & 4.155 & 242 \\ 249 & \mathrm{U} & \text { none } & & & & \\ & \mathrm{V} & \text { none } & & & & \\ 250 & \mathrm{U} & \text { none } & & & & \\ & \mathrm{V} & \text { none } & & & & \\ 251 & \mathrm{U} & 3.665 & 46.865 & 0.782 & 6.846 & 121 \\ & \mathrm{~V} & 6.260 & 69.274 & 0.642 & 8.323 & 121 \\ 252 & \mathrm{U} & -12.984 & 16.989 & 0.092 & 4.122 & 49 \\ & \mathrm{~V} & 4.286 & 55.585 & 0.763 & 7.456 & 49\end{array}$


Table 6: Mapped Atlas SASS wind statistics - 1-day vector average plus direction bins (wind blowing toward a given direction)

\begin{tabular}{|c|c|c|c|c|c|c|c|c|c|c|}
\hline DAY & MEAN & VARSPD & SDSPD & VARVEC & SDVEC & $\mathrm{N}$ & NORTH & EAST & SOUTH & WEST \\
\hline 224 & 6.130 & 5.743 & 2.396 & 36.901 & 6.075 & 378 & 0.29 & 0.26 & 0.00 & 0.45 \\
\hline 225 & 6.074 & 5.205 & 2.282 & 35.490 & 5.957 & 340 & 0.22 & 0.18 & 0.04 & 0.56 \\
\hline 226 & 6.426 & 6.025 & 2.455 & 40.690 & 6.379 & 463 & 0.19 & 0.18 & 0.19 & 0.43 \\
\hline 227 & 6.701 & 11.767 & 3.430 & 54.077 & 7.354 & 499 & 0.12 & 0.44 & 0.18 & 0.26 \\
\hline 228 & 6.838 & 13.910 & 3.730 & 51.314 & 7.163 & 453 & 0.08 & 0.56 & 0.14 & 0.23 \\
\hline 229 & 5.236 & 5.676 & 2.382 & 32.251 & 5.679 & 631 & 0.31 & 0.25 & 0.14 & 0.30 \\
\hline 230 & 4.974 & 4.590 & 2.142 & 27.081 & 5.204 & 695 & 0.36 & 0.25 & 0.06 & 0.33 \\
\hline 231 & 4.865 & 2.718 & 1.649 & 8.900 & 2.983 & 134 & 0.43 & 0.53 & 0.04 & 0.00 \\
\hline 232 & 6.343 & 7.691 & 2.773 & 46.271 & 6.802 & 466 & 0.24 & 0.35 & 0.13 & 0.28 \\
\hline 233 & 6.488 & 8.960 & 2.993 & 48.764 & 6.983 & 636 & 0.14 & 0.35 & 0.28 & 0.23 \\
\hline 234 & 5.612 & 7.369 & 2.715 & 38.473 & 6.203 & 623 & 0.16 & 0.27 & 0.28 & 0.29 \\
\hline 235 & 5.941 & 7.468 & 2.733 & 40.033 & 6.327 & 662 & 0.32 & 0.32 & 0.10 & 0.26 \\
\hline 236 & 5.878 & 9.507 & 3.083 & 38.031 & 6.167 & 693 & 0.35 & 0.35 & 0.15 & 0.16 \\
\hline 237 & 4.642 & 2.656 & 1.630 & 16.763 & 4.094 & 206 & 0.02 & 0.39 & 0.44 & 0.15 \\
\hline 238 & 7.023 & 7.578 & 2.753 & 46.123 & 6.791 & 545 & 0.42 & 0.21 & 0.07 & 0.30 \\
\hline 239 & 6.342 & 6.559 & 2.561 & 32.762 & 5.724 & 372 & 0.51 & 0.11 & 0.01 & 0.38 \\
\hline 240 & 4.955 & 5.040 & 2.245 & 18.987 & 4.357 & 412 & 0.25 & 0.07 & 0.02 & 0.66 \\
\hline 241 & 5.891 & 7.785 & 2.790 & 33.716 & 5.807 & 453 & 0.17 & 0.04 & 0.17 & 0.62 \\
\hline 242 & 6.450 & 8.149 & 2.855 & 36.337 & 6.028 & 260 & 0.08 & 0.03 & 0.38 & 0.52 \\
\hline 243 & 5.101 & 3.873 & 1.968 & 20.527 & 4.531 & 98 & 0.07 & 0.08 & 0.32 & 0.53 \\
\hline 244 & 5.195 & 7.518 & 2.742 & 30.127 & 5.489 & 340 & 0.29 & 0.09 & 0.29 & 0.33 \\
\hline 245 & 5.051 & 5.083 & 2.254 & 19.744 & 4.443 & 255 & 0.05 & 0.08 & 0.35 & 0.51 \\
\hline 246 & 6.575 & 11.205 & 3.347 & 49.952 & 7.068 & 378 & 0.18 & 0.12 & 0.25 & 0.45 \\
\hline 247 & 7.227 & 11.487 & 3.389 & 55.679 & 7.462 & 612 & 0.41 & 0.09 & 0.16 & 0.34 \\
\hline 248 & 7.981 & 21.153 & 4.599 & 66.462 & 8.152 & 920 & 0.45 & 0.22 & 0.09 & 0.23 \\
\hline 249 & 6.814 & 10.161 & 3.188 & 53.615 & 7.322 & 423 & 0.22 & 0.23 & 0.16 & 0.40 \\
\hline 250 & 6.316 & 8.699 & 2.949 & 46.994 & 6.855 & 257 & 0.12 & 0.30 & 0.21 & 0.37 \\
\hline 251 & 8.458 & 18.499 & 4.301 & 77.959 & 8.829 & 404 & 0.43 & 0.23 & 0.15 & 0.19 \\
\hline 252 & 9.033 & 31.664 & 5.627 & 108.209 & 10.402 & 317 & 0.33 & 0.17 & 0.19 & 0.31 \\
\hline
\end{tabular}


Table 7: Mapped Atlas SASS wind statistics - 3-day average plus direction bins

(wind blowing toward a given direction)

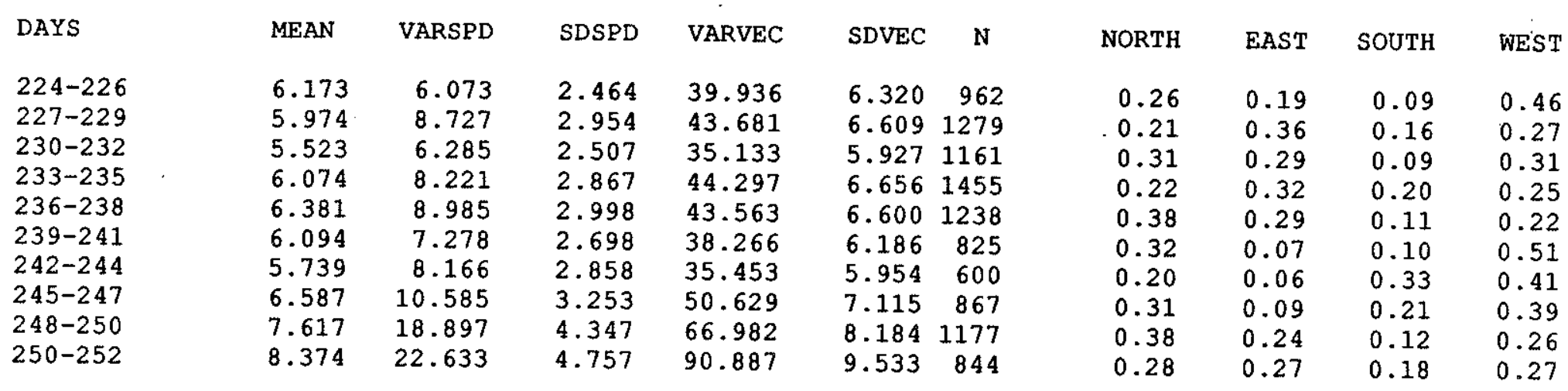


Table 8: FNOC component wind statistics - area averages

U Component

\begin{tabular}{|c|c|c|c|c|c|c|c|}
\hline $\mathrm{DAY} / \mathrm{T}$ & $\mathrm{CME}$ & & $\begin{array}{l}\text { MEAN } \\
(\mathrm{m} / \mathrm{s})\end{array}$ & $\begin{array}{c}\text { VAR } \\
\left(m^{\wedge} 2 / s^{\wedge} 2\right)\end{array}$ & WGTVAR & $\begin{array}{l}\text { SDEV } \\
(\mathrm{m} / \mathrm{s})\end{array}$ & $\mathrm{N}$ \\
\hline 224 & 00 & U1 & 2.545 & 3.364 & 0.344 & 1.834 & 49 \\
\hline & & $\mathrm{U} 2$ & -3.856 & 0.386 & 0.025 & 0.621 & 36 \\
\hline 224 & 12 & U1 & -7.366 & 1.021 & 0.018 & 1.010 & 36 \\
\hline & & U2 & -2.861 & 2.862 & 0.260 & 1.692 & 64 \\
\hline & & U3 & 2.679 & 2.679 & 0.178 & 1.637 & 36 \\
\hline 224 & $12 \mathrm{~B}$ & U1 & -0.010 & 3.531 & 1.029 & 1.879 & 36 \\
\hline 225 & 00 & U1 & 8.389 & 2.700 & 0.037 & 1.643 & 36 \\
\hline & & $\mathrm{U} 2$ & 1.747 & 1.980 & 0.392 & 1.393 & 49 \\
\hline & & U3 & -3.904 & 0.898 & 0.056 & 0.948 & 36 \\
\hline 225 & 12 & U1 & 1.151 & 1.946 & 0.602 & 1.395 & 49 \\
\hline 225 & $12 \mathrm{~B}$ & $\mathrm{U1}$ & -6.839 & 0.567 & 0.012 & 0.753 & 36 \\
\hline & & $\mathrm{U} 2$ & -1.600 & 15.450 & 0.879 & 3.931 & 36 \\
\hline 226 & 00 & U1 & -1.679 & 3.442 & 0.556 & 1.855 & 49 \\
\hline & & $\mathrm{U} 2$ & -6.840 & 1.363 & 0.028 & 1.167 & 49 \\
\hline 226 & 12 & U1 & -0.724 & 2.306 & 0.834 & 1.518 & 36 \\
\hline 226 & $12 \mathrm{~B}$ & U1 & -7.656 & 3.595 & 0.058 & 1.896 & 49 \\
\hline & & $\mathrm{U} 2$ & 0.993 & 4.948 & 0.854 & 2.224 & 36 \\
\hline & & U3 & 5.461 & 0.828 & 0.027 & 0.910 & 36 \\
\hline 227 & 00 & U1 & -4.194 & 3.963 & 0.184 & 1.991 & 100 \\
\hline 227 & $0 O B$ & U1 & 3.048 & 0.456 & 0.047 & 0.675 & 36 \\
\hline & & $\mathrm{U} 2$ & 1.424 & 1.940 & 0.496 & 1.393 & 36 \\
\hline & & U3 & -5.128 & 2.171 & 0.076 & 1.473 & 36 \\
\hline 227 & 12 & U1 & -4.642 & 5.093 & 0.192 & 2.257 & 36 \\
\hline & & $\mathrm{U} 2$ & 0.547 & 0.081 & 0.214 & 0.285 & 36 \\
\hline 228 & 00 & U1 & 12.509 & 5.660 & 0.035 & 2.379 & 49 \\
\hline & & $\mathrm{U} 2$ & 8.149 & 2.325 & 0.034 & 1.525 & 49 \\
\hline & & U3 & 2.342 & 9.411 & 0.643 & 3.068 & 36 \\
\hline & & $\mathrm{U} 4$ & -4.650 & 3.755 & 0.148 & 1.938 & 49 \\
\hline 228 & $O O B$ & $\mathrm{U1}$ & 0.918 & 0.625 & 0.431 & 0.791 & 36 \\
\hline 228 & 12 & U1 & 4.249 & 1.663 & 0.084 & 1.289 & 64 \\
\hline 228 & $12 \mathrm{~B}$ & U1 & -4.985 & 5.404 & 0.180 & 2.325 & 36 \\
\hline & & $\mathrm{U} 2$ & 1.611 & 3.579 & 0.587 & 1.892 & 49 \\
\hline 229 & 00 & $\mathrm{U1}$ & 1.375 & 1.763 & 0.487 & 1.328 & 49 \\
\hline & & $\mathrm{U} 2$ & -3.573 & 5.119 & 0.289 & 2.263 & 36 \\
\hline 229 & 12 & U1 & 9.977 & 11.068 & 0.100 & 3.327 & 36 \\
\hline 229 & $12 \mathrm{~B}$ & U1 & -2.432 & 4.303 & 0.425 & 2.074 & 49 \\
\hline & & U2 & 2.416 & 1.583 & 0.214 & 1.258 & 49 \\
\hline & & U3 & 1.293 & 0.942 & 0.363 & 0.971 & 49 \\
\hline 230 & 00 & U1 & 3.658 & 25.670 & 0.661 & 5.067 & 121 \\
\hline & & U2 & 0.908 & 4.895 & 0.862 & 2.213 & 121 \\
\hline & & U3 & -3.336 & 14.368 & 0.566 & 3.790 & 121 \\
\hline 230 & 12 & U1 & -3.864 & 10.684 & 0.419 & 3.269 & 121 \\
\hline & & $\mathrm{U} 2$ & 0.641 & 1.494 & 0.797 & 1.222 & 49 \\
\hline & & U3 & 3.714 & 5.027 & 0.268 & 2.242 & 64 \\
\hline 230 & $12 \mathrm{~B}$ & U1 & 0.878 & 6.530 & 0.911 & 2.555 & 49 \\
\hline 231 & 00 & $\mathrm{u} 1$ & 3.290 & 15.740 & 0.600 & 3.967 & 49 \\
\hline 232 & 00 & U1 & 4.540 & 1.136 & 0.052 & 1.066 & 49 \\
\hline 232 & $00 B$ & U1 & 3.199 & 1.215 & 0.106 & 1.102 & 36 \\
\hline 232 & 12 & $\mathrm{U1}$ & -4.410 & 1.784 & 0.084 & 1.336 & 49 \\
\hline & & $\mathrm{U} 2$ & 2.176 & 10.528 & 0.700 & 3.245 & 49 \\
\hline
\end{tabular}




\begin{tabular}{|c|c|c|c|c|c|c|c|}
\hline & & U3 & 6.399 & 4.770 & 0.105 & 2.184 & 49 \\
\hline 232 & $12 \mathrm{~B}$ & U1 & -2.756 & 2.913 & 0.279 & 1.707 & \\
\hline \multirow[t]{4}{*}{233} & 00 & U1 & 2.748 & 9.540 & 0.563 & 3.089 & 6 \\
\hline & & U2 & 1.518 & 9.978 & 0.831 & 3.159 & \\
\hline & & U3 & 4.629 & 4.263 & 0.166 & 2.065 & 45 \\
\hline & & U4 & -3.570 & 5.603 & 0.307 & 2.367 & 4 \\
\hline \multirow[t]{2}{*}{233} & 12 & U1 & 5.409 & 3.799 & 0.115 & 1.949 & \\
\hline & & U2 & 2.042 & 4.779 & 0.542 & 2.186 & \\
\hline \multirow[t]{2}{*}{233} & $12 \mathrm{~B}$ & U1 & -4.325 & 2.326 & 0.111 & 1.525 & 3 \\
\hline & & U2 & 5.099 & 5.354 & 0.171 & 2.314 & \\
\hline \multirow[t]{4}{*}{234} & 00 & U1 & 2.693 & 8.920 & 0.560 & 2.987 & 3 \\
\hline & & U2 & -2.256 & 24.208 & 0.846 & 4.920 & 3 \\
\hline & & U3 & 3.269 & 1.963 & 0.156 & 1.401 & 4 \\
\hline & & U4 & -2.725 & 5.952 & 0.449 & 2.440 & \\
\hline \multirow[t]{2}{*}{234} & OOB & U1 & 1.235 & 9.892 & 0.882 & 3.145 & 4 \\
\hline & & U2 & -1.242 & 5.173 & 0.783 & 2.274 & \\
\hline 234 & 12 & U1 & -1.376 & 10.786 & 0.871 & 3.284 & 3 \\
\hline \multirow[t]{3}{*}{234} & $12 \mathrm{~B}$ & $\mathrm{U} 1$ & -3.548 & 4.064 & 0.245 & 2.016 & 4 \\
\hline & & U2 & 1.890 & 5.467 & 0.615 & 2.338 & \\
\hline & & U3 & -1.188 & 2.016 & 0.598 & 1.420 & 3 \\
\hline 235 & 00 & U1 & -4.249 & 1.461 & 0.075 & 1.209 & 3 \\
\hline \multirow{3}{*}{235} & $O O B$ & U1 & -3.123 & 30.521 & 0.770 & 5.525 & \\
\hline & & U2 & 2.203 & 7.622 & 0.617 & 2.761 & \\
\hline & & U3 & -0.664 & 8.981 & 0.972 & 2.997 & 4 \\
\hline \multirow[t]{3}{*}{235} & 12 & U1 & -2.819 & 4.108 & 0.343 & 2.207 & \\
\hline & & U2 & 1.796 & 2.226 & 0.412 & 1.492 & \\
\hline & & U3 & 4.612 & 11.261 & 0.348 & 3.356 & \\
\hline 235 & $12 \mathrm{~B}$ & U1 & -2.036 & 0.774 & 0.158 & 0.880 & \\
\hline \multirow[t]{3}{*}{236} & 00 & U1 & 0.720 & 33.438 & 0.993 & 5.783 & 12 \\
\hline & & U2 & 4.902 & 8.663 & 0.266 & 2.943 & \\
\hline & & U3 & 0.039 & 2.235 & 1.015 & 1.495 & 6 \\
\hline \multirow[t]{2}{*}{236} & 12 & U1 & 4.508 & 13.433 & 0.402 & 3.665 & \\
\hline & & U2 & 1.904 & 32.370 & 0.906 & 5.689 & 12 \\
\hline 2.36 & $12 \mathrm{~B}$ & U1 & 0.116 & 2.075 & 1.009 & 1.441 & 6 \\
\hline \multirow[t]{2}{*}{237} & 00 & U1 & 5.729 & 0.920 & 0.027 & 0.959 & \\
\hline & & $\mathrm{U} 2$ & -1.135 & 10.234 & 0.905 & 3.199 & \\
\hline 237 & $00 \mathrm{~B}$ & U1 & -0.255 & 4.369 & 0.993 & 2.090 & 12 \\
\hline \multirow[t]{4}{*}{238} & 12 & U1 & -3.856 & 2.811 & 0.159 & 1.677 & \\
\hline & & U2 & 2.248 & 1.911 & 0.275 & 1.382 & \\
\hline & & U3 & -4.194 & 8.327 & 0.323 & 2.886 & \\
\hline & & U4 & -0.760 & 13.474 & 0.985 & 3.671 & \\
\hline 238 & $12 \mathrm{~B}$ & U1 & 1.920 & 1.755 & 0.325 & 1.325 & 4 \\
\hline \multirow[t]{3}{*}{239} & 00 & U1 & -6.168 & 11.113 & 0.227 & 3.334 & \\
\hline & & $\mathrm{U} 2$ & 5.407 & 1.293 & 0.042 & 1.137 & \\
\hline & & U3 & -1.207 & 6.974 & 0.829 & 2.607 & 12. \\
\hline \multirow[t]{2}{*}{240} & 00 & UI & 2.462 & 8.271 & 0.586 & 2.876 & \\
\hline & & U2 & -3.922 & 8.973 & 0.371 & 2.995 & 4 \\
\hline 240 & OOB & U1 & -2.341 & 0.379 & 0.065 & 0.615 & 3 \\
\hline \multirow{3}{*}{241} & 00 & U1 & -2.938 & 8.201 & 0.489 & 2.864 & 12 \\
\hline & $\mathrm{OOB}$ & $\mathrm{U} 1$ & -0.682 & 3.496 & 0.899 & 1.870 & 4 \\
\hline & & U2 & -3.014 & 2.538 & 0.219 & 1.593 & 12 \\
\hline \multirow[t]{3}{*}{242} & 00 & U1 & -3.324 & 1.853 & 0.144 & 1.361 & \\
\hline & & U2 & -3.050 & 0.582 & 0.059 & 0.763 & 4 \\
\hline & & U3 & -0.312 & 4.986 & 1.001 & 2.233 & 4 \\
\hline 243 & 00 & U1 & 0.957 & 6.831 & 0.898 & 2.614 & 4 \\
\hline & & U2 & -3.768 & 3.037 & 0.177 & 1.743 & 4 \\
\hline 244 & 12 & U1 & -4.521 & 15.699 & 0.440 & 3.962 & 36 \\
\hline & & U2 & 0.732 & 2.860 & 0.862 & 1.691 & 3 \\
\hline
\end{tabular}




\begin{tabular}{|c|c|c|c|c|c|c|c|}
\hline & & U3 & 1.521 & 0.989 & 0.302 & 0.994 & 36 \\
\hline \multirow[t]{2}{*}{244} & $12 \mathrm{~B}$ & U1 & -1.089 & 36.305 & 0.988 & 6.025 & 49 \\
\hline & & U2 & -6.862 & 131.218 & 0.747 & 11.455 & 49 \\
\hline \multirow[t]{3}{*}{245} & 00 & U1 & 1.546 & 1.006 & 0.298 & 1.003 & 49 \\
\hline & & U2 & 0.192 & 1.548 & 1.004 & 1.244 & 36 \\
\hline & & U3 & -6.041 & 5.442 & 0.130 & 2.333 & 49 \\
\hline \multirow[t]{3}{*}{246} & 00 & U1 & 4.887 & 5.362 & 0.184 & 2.316 & 36 \\
\hline & & U2 & -0.125 & 1.647 & 1.019 & 1.283 & 36 \\
\hline & & U3 & -5.280 & 0.832 & 0.029 & 0.912 & 49 \\
\hline 247 & 00 & U1 & -3.287 & 9.251 & 0.463 & 3.042 & 121 \\
\hline \multirow[t]{2}{*}{247} & $O O B$ & U1 & 2.481 & 139.787 & 0.984 & 11.823 & 36 \\
\hline & & U2 & -0.072 & 12.236 & 1.010 & 3.498 & 100 \\
\hline \multirow[t]{3}{*}{248} & 00 & U1 & 6.185 & 1.376 & 0.035 & 1.173 & 121 \\
\hline & & U2 & 6.664 & 10.889 & 0.198 & 3.300 & 49 \\
\hline & & U3 & -1.764 & 10.913 & 0.783 & 3.303 & 121 \\
\hline 248 & $O O B$ & U1 & 3.092 & 2.486 & 0.207 & 1.577 & 64 \\
\hline \multirow[t]{2}{*}{248} & 12 & U1 & 5.367 & 14.806 & 0.343 & 3.848 & 36 \\
\hline & & U2 & 7.190 & 47.829 & 0.482 & 6.916 & 121 \\
\hline \multirow[t]{2}{*}{248} & $12 \mathrm{~B}$ & U1 & -1.940 & 3.151 & 0.457 & 1.775 & 121 \\
\hline & & $\mathrm{U} 2$ & 3.285 & 3.003 & 0.218 & 1.733 & 121 \\
\hline \multirow[t]{2}{*}{249} & 00 & U1 & 4.551 & 11.445 & 0.359 & 3.383 & 49 \\
\hline & & $\mathrm{U} 2$ & -4.974 & 12.419 & 0.336 & 3.524 & 81 \\
\hline 249 & $O O B$ & U1 & -1.615 & 1.141 & 0.307 & 1.068 & 36 \\
\hline 250 & 00 & U1 & -4.298 & 10.430 & 0.362 & 3.230 & 121 \\
\hline \multirow[t]{3}{*}{250} & $O O B$ & U1 & 4.874 & 2.812 & 0.106 & 1.677 & 64 \\
\hline & & บ2 & -2.317 & 1.454 & 0.214 & 1.206 & 36 \\
\hline & & U3 & -3.803 & 0.642 & 0.043 & 0.801 & 36 \\
\hline 251 & 12 & U1 & 5.354 & 34.252 & 0.547 & 5.852 & 121 \\
\hline \multirow{2}{*}{251} & $12 \mathrm{~B}$ & U1 & -3.517 & 16.317 & 0.572 & 4.039 & 100 \\
\hline & & U2 & 2.407 & 11.552 & 0.675 & 3.399 & 49 \\
\hline \multirow[t]{2}{*}{252} & 00 & U1 & 2.614 & 16.148 & 0.713 & 4.018 & 49 \\
\hline & & U2 & -3.517 & 4.905 & 0.286 & 2.215 & 49 \\
\hline 252 & $O O B$ & U1 & -0.607 & 1.176 & 0.778 & 1.084 & 36 \\
\hline \multirow[t]{4}{*}{2.53} & 00 & U1 & -10.485 & 18.268 & 0.143 & 4.274 & 49 \\
\hline & & $\mathrm{U} 2$ & 5.566 & 51.617 & 0.633 & 7.185 & 49 \\
\hline & & U3 & 4.852 & 10.822 & 0.317 & 3.290 & 49 \\
\hline & & U4 & -1.753 & 3.670 & 0.553 & 1.916 & 36 \\
\hline
\end{tabular}

V Component

\begin{tabular}{|c|c|c|c|c|c|c|c|}
\hline \multicolumn{3}{|c|}{ DAY/TIME } & $\begin{array}{l}\text { MEAN } \\
(\mathrm{m} / \mathrm{s})\end{array}$ & $\begin{array}{c}\text { VAR } \\
\left(m^{\wedge} 2 / s^{\wedge} 2\right)\end{array}$ & WGTVAR & $\begin{array}{l}\text { SDEV } \\
(\mathrm{m} / \mathrm{s})\end{array}$ & $\mathrm{N}$ \\
\hline \multirow[t]{2}{*}{224} & 00 & v1 & 2.310 & 0.449 & 0.078 & 0.670 & 49 \\
\hline & & v2 & 0.174 & 0.700 & 0.985 & 0.837 & 36 \\
\hline \multirow[t]{3}{*}{224} & 12 & $\mathrm{~V} 1$ & -0.962 & 0.657 & 0.420 & 0.811 & 36 \\
\hline & & v2 & 0.776 & 0.492 & 0.453 & 0.701 & \\
\hline & & v3 & 2.274 & 4.548 & 0.474 & 2.132 & 36 \\
\hline 224 & $12 \mathrm{~B}$ & V1 & 2.313 & 4.545 & 0.465 & 2.132 & 36 \\
\hline \multirow[t]{3}{*}{225} & 00 & V1 & 0.041 & 0.505 & 1.025 & 0.710 & 36 \\
\hline & & V2 & 3.352 & 1.583 & 0.124 & 1.258 & 49 \\
\hline & & V3 & -0.401 & 0.114 & 0.419 & 0.337 & 36 \\
\hline 225 & 12 & $\mathrm{~V} 1$ & 2.804 & 1.014 & 0.115 & 1.007 & 49 \\
\hline \multirow[t]{2}{*}{225} & $12 \mathrm{~B}$ & V1 & -1.067 & 2.058 & 0.655 & 1.435 & 36 \\
\hline & & v2 & 4.481 & 4.773 & 0.193 & 2.185 & 36 \\
\hline 226 & 00 & V1 & -2.159 & 4.537 & 0.498 & 2.130 & 49 \\
\hline
\end{tabular}




\begin{tabular}{|c|c|c|c|c|c|c|c|}
\hline & & V2 & -0.983 & 2.165 & 0.701 & 1.471 & 49 \\
\hline 226 & 12 & V1 & 8.182 & 0.394 & 0.006 & 0.627 & 36 \\
\hline \multirow[t]{3}{*}{226} & $12 \mathrm{~B}$ & V1 & -0.805 & 3.495 & 0.858 & 1.870 & 4 \\
\hline & & V2 & 2.557 & 3.711 & 0.366 & 1.926 & \\
\hline & & V3 & 3.166 & 1.203 & 0.107 & 1.097 & \\
\hline 227 & 00 & V1 & -4.025 & 5.667 & 0.260 & 2.380 & 100 \\
\hline \multirow{3}{*}{227} & $O O B$ & V1 & -0.692 & 0.526 & 0.531 & 0.725 & \\
\hline & & V2 & 1.235 & 0.306 & 0.168 & 0.553 & \\
\hline & & v3 & -0.483 & 1.027 & 0.834 & 1.013 & 3 \\
\hline \multirow[t]{2}{*}{227} & 12 & V1 & 1.170 & 0.254 & 0.157 & 0.504 & \\
\hline & & V2 & 1.058 & 0.041 & 0.035 & 0.203 & 3 \\
\hline \multirow[t]{4}{*}{228} & 00 & V1 & -3.708 & 23.905 & 0.643 & 4.889 & 4 \\
\hline & & V2 & 1.208 & 4.902 & 0.783 & 2.214 & 4 \\
\hline & & v3 & 0.957 & 1.881 & 0.685 & 1.372 & \\
\hline & & V4 & -0.871 & 1.920 & 0.727 & 1.386 & \\
\hline 228 & $O O B$ & V1 & 1.160 & 0.465 & 0.259 & 0.682 & 3 \\
\hline 228 & 12 & $\mathrm{v} 1$ & 0.145 & 3.205 & 1.009 & 1.790 & \\
\hline \multirow[t]{2}{*}{228} & $12 \mathrm{~B}$ & $\mathrm{~V} 1$ & -0.041 & 1.008 & 1.027 & 1.004 & 3 \\
\hline & & v2 & 1.833 & 1.287 & 0.279 & 1.134 & 4 \\
\hline \multirow[t]{2}{*}{229} & 00 & v1 & 0.854 & 1.121 & 0.613 & 1.059 & \\
\hline & & V2 & -1.563 & 3.842 & 0.622 & 1.960 & 3 \\
\hline 229 & 12 & V1 & -2.162 & 8.450 & 0.656 & 2.907 & \\
\hline \multirow{3}{*}{229} & $12 \mathrm{~B}$ & v1 & -0.077 & 3.154 & 1.019 & 1.776 & \\
\hline & & v2 & 0.383 & 1.578 & 0.932 & 1.256 & 4 \\
\hline & & v3 & 4.355 & 1.328 & 0.066 & 1.152 & 4 \\
\hline \multirow[t]{3}{*}{230} & 00 & V1 & 2.421 & 7.333 & 0.558 & 2.708 & 12 \\
\hline & & v2 & 6.364 & 1.535 & 0.037 & 1.239 & 12 \\
\hline & & v3 & 1.800 & 2.518 & 0.439 & 1.587 & 12 \\
\hline \multirow[t]{3}{*}{230} & 12 & VI & -0.011 & 1.530 & 1.008 & 1.237 & 12 \\
\hline & & v2 & 1.640 & 0.394 & 0.128 & 0.627 & \\
\hline & & v3 & 4.502 & 4.151 & 0.170 & 2.037 & \\
\hline 230 & $12 \mathrm{~B}$ & v1 & 3.339 & 4.864 & 0.306 & 2.205 & \\
\hline 231 & 00 & $\mathrm{~V} 1$ & 5.297 & 2.169 & 0.072 & 1.473 & \\
\hline $23 ̧ 2$ & 00 & V1 & 3.285 & 0.927 & 0.079 & 0.963 & \\
\hline 232 & $O O B$ & $\mathrm{~V} 1$ & -0.216 & 1.661 & 1.000 & 1.289 & \\
\hline \multirow[t]{3}{*}{232} & 12 & v1 & -0.538 & 0.594 & 0.682 & 0.771 & \\
\hline & & V2 & 2.722 & 3.045 & 0.293 & 1.745 & \\
\hline & & v3 & 1.972 & 6.600 & 0.637 & 2.569 & \\
\hline 232 & $12 \mathrm{~B}$ & V1 & 2.912 & 1.819 & 0.178 & 1.349 & \\
\hline \multirow[t]{4}{*}{233} & 00 & v1 & -1.560 & 24.322 & 0.922 & 4.932 & \\
\hline & & v2 & 1.760 & 6.661 & 0.696 & 2.581 & \\
\hline & & v3 & 4.236 & 3.818 & 0.176 & 1.954 & \\
\hline & & V4 & 2.037 & 0.975 & 0.191 & 0.987 & \\
\hline \multirow[t]{2}{*}{233} & 12 & V1 & -2.264 & 0.900 & 0.150 & 0.949 & \\
\hline & & v2 & -1.151 & 16.873 & 0.952 & 4.108 & \\
\hline \multirow[t]{2}{*}{233} & $12 \mathrm{~B}$ & VI & 1.630 & 0.564 & 0.176 & 0.751 & 3 \\
\hline & & V2 & 1.455 & 6.325 & 0.761 & 2.515 & \\
\hline \multirow[t]{4}{*}{234} & 00 & VI & -2.383 & 17.691 & 0.773 & 4.206 & \\
\hline & & $\mathrm{v} 2$ & 4.375 & 6.128 & 0.244 & 2.475 & \\
\hline & & V3 & 0.862 & 6.364 & 0.912 & 2.523 & \\
\hline & & V4 & -0.716 & 2.278 & 0.830 & 1.509 & \\
\hline \multirow[t]{2}{*}{234} & $O O B$ & V1 & -1.050 & 11.943 & 0.933 & 3.456 & 4 \\
\hline & & V2 & 1.277 & 0.763 & 0.321 & 0.873 & 4 \\
\hline 234 & 12 & $\mathrm{~V} 1$ & 2.027 & 5.106 & 0.563 & 2.260 & \\
\hline \multirow[t]{3}{*}{234} & $12 \mathrm{~B}$ & V1 & 0.107 & 5.330 & 1.019 & 2.309 & 4 \\
\hline & & $\mathrm{v} 2$ & 2.248 & 0.926 & 0.156 & 0.962 & 3 \\
\hline & & v3 & -1.403 & 10.279 & 0.859 & 3.206 & 3 \\
\hline & 00 & V1 & -2.088 & 0.659 & 0.132 & 0.812 & 3 \\
\hline
\end{tabular}




\begin{tabular}{|c|c|c|c|c|c|c|c|}
\hline \multirow[t]{3}{*}{235} & OOB & V1 & 2.257 & 19.478 & 0.806 & 4.413 & 49 \\
\hline & & V2 & -0.384 & 6.699 & 0.994 & 2.588 & 64 \\
\hline & & v3 & 1.516 & 1.701 & 0.429 & 1.304 & 49 \\
\hline \multirow[t]{3}{*}{235} & 12 & V1 & -0.014 & 1.620 & 1.021 & 1.273 & 49 \\
\hline & & v2 & 2.538 & 0.601 & 0.085 & 0.775 & 49 \\
\hline & & V3 & 1.446 & 20.677 & 0.921 & 4.547 & 64 \\
\hline 235 & $12 \mathrm{~B}$ & V1 & -1.027 & 6.191 & 0.875 & 2.488 & 36 \\
\hline \multirow[t]{3}{*}{236} & 00 & V1 & 2.267 & 19.855 & 0.800 & 4.456 & 121 \\
\hline & & $\mathrm{V} 2$ & 1.683 & 2.362 & 0.457 & 1.537 & 81 \\
\hline & & v3 & 1.706 & 1.687 & 0.369 & 1.299 & 64 \\
\hline \multirow[t]{2}{*}{236} & 12 & v1 & 1.780 & 0.202 & 0.060 & 0.449 & 36 \\
\hline & & v2 & 6.065 & 11.968 & 0.246 & 3.459 & 121 \\
\hline 236 & $12 \mathrm{~B}$ & V1 & -0.191 & 2.257 & 0.999 & 1.502 & 64 \\
\hline \multirow[t]{2}{*}{237} & 00 & V1 & 1.109 & 3.108 & 0.731 & 1.763 & 36 \\
\hline & & v2 & 0.445 & 2.926 & 0.955 & 1.711 & 49 \\
\hline 237 & $O O B$ & V1 & 0.372 & 1.085 & 0.893 & 1.042 & 121 \\
\hline \multirow[t]{4}{*}{238} & 12 & V1 & 1.347 & 8.670 & 0.838 & 2.945 & 64 \\
\hline & & v2 & 4.461 & 2.523 & 0.113 & 1.588 & 64 \\
\hline & & V3 & 3.762 & 2.685 & 0.160 & 1.639 & 81 \\
\hline & & V4 & 1.924 & 5.247 & 0.596 & 2.291 & 36 \\
\hline 238 & $12 \mathrm{~B}$ & V1 & -0.262 & 1.929 & 0.985 & 1.389 & 49 \\
\hline \multirow[t]{3}{*}{239} & 00 & V1 & 5.269 & 6.788 & 0.197 & 2.605 & 81 \\
\hline & & v2 & 5.236 & 0.636 & 0.023 & 0.797 & 49 \\
\hline & & v3 & 3.269 & 2.279 & 0.176 & 1.510 & 121 \\
\hline \multirow[t]{2}{*}{240} & 00 & V1 & 4.932 & 12.467 & 0.342 & 3.531 & 36 \\
\hline & & v2 & 1.378 & 5.943 & 0.770 & 2.438 & 49 \\
\hline 240 & $\mathrm{OOB}$ & V1 & -0.477 & 1.026 & 0.837 & 1.013 & 36 \\
\hline 241 & 00 & V1 & -1.220 & 7.554 & 0.841 & 2.748 & 121 \\
\hline \multirow{2}{*}{241} & $O O B$ & V1 & 6.402 & 18.109 & 0.308 & 4.255 & 49 \\
\hline & & V2 & 0.492 & 3.819 & 0.948 & 1.954 & 121 \\
\hline \multirow[t]{3}{*}{242} & 00 & V1 & -3.780 & 7.242 & 0.338 & 2.691 & 64 \\
\hline & & v2 & -0.245 & 2.582 & 0.997 & 1.607 & 49 \\
\hline & & v3 & 1.054 & 8.491 & 0.900 & 2.914 & 49 \\
\hline \multirow[t]{2}{*}{243} & 00 & V1 & 2.004 & 3.668 & 0.482 & 1.915 & 49 \\
\hline & & $\mathrm{V} 2$ & 0.470 & 2.489 & 0.936 & 1.578 & 49 \\
\hline \multirow[t]{3}{*}{244} & 12 & V1 & -1.237 & 3.503 & 0.710 & 1.872 & 36 \\
\hline & & V2 & 0.685 & 0.470 & 0.507 & 0.686 & 36 \\
\hline & & v3 & 2.377 & 0.220 & 0.037 & 0.469 & 36 \\
\hline \multirow[t]{2}{*}{244} & $12 \mathrm{~B}$ & VI & 2.864 & 15.479 & 0.662 & 3.934 & 49 \\
\hline & & V2 & 3.846 & 105.889 & 0.893 & 10.290 & 49 \\
\hline \multirow[t]{3}{*}{245} & 00 & v1 & 1.575 & 5.215 & 0.687 & 2.284 & 49 \\
\hline & & V2 & 1.145 & 0.258 & 0.165 & 0.508 & 36 \\
\hline & & v3 & 0.141 & 2.401 & 1.012 & 1.550 & 49 \\
\hline \multirow[t]{3}{*}{246} & 00 & $\mathrm{~V} 1$ & 1.012 & 4.576 & 0.836 & 2.139 & 36 \\
\hline & & V2 & 0.313 & 1.176 & 0.947 & 1.084 & 36 \\
\hline & & v3 & -0.042 & 0.552 & 1.018 & 0.743 & 49 \\
\hline 247 & 00 & V1 & -2.579 & 6.768 & 0.506 & 2.602 & 121 \\
\hline \multirow[t]{2}{*}{247} & OOB & v1 & 7.182 & 104.769 & 0.683 & 10.236 & 36 \\
\hline & & V2 & 3.145 & 2.963 & 0.231 & 1.721 & 100 \\
\hline 248 & 00 & v1 & 9.310 & 4.477 & 0.049 & 2.116 & 121 \\
\hline & & v2 & 7.021 & 3.441 & 0.065 & 1.855 & 49 \\
\hline & & v3 & 1.764 & 3.306 & 0.517 & 1.818 & 121 \\
\hline 248 & $\mathrm{OOB}$ & V1 & -0.711 & 4.335 & 0.908 & 2.082 & 64 \\
\hline 248 & 12 & V1 & 8.241 & 7.761 & 0.103 & 2.786 & 36 \\
\hline & & $\mathrm{V} 2$ & 7.697 & 44.395 & 0.430 & 6.663 & 121 \\
\hline 248 & $12 \mathrm{~B}$ & V1 & 2.702 & 0.729 & 0.091 & 0.854 & 1.21 \\
\hline & & v2 & -0.096 & 5.535 & 1.007 & 2.353 & 1.21 \\
\hline 249 & 00 & V1 & 5.569 & 10.666 & 0.257 & 3.266 & 49 \\
\hline
\end{tabular}




\begin{tabular}{|c|c|c|c|c|c|c|c|}
\hline & & v2 & 0.019 & 16.876 & 1.012 & 4.108 & 81 \\
\hline 249 & $O O B$ & VI & 2.009 & 6.905 & 0.642 & 2.628 & 36 \\
\hline 250 & 00 & V1 & -0.768 & 4.170 & 0.883 & 2.042 & 121 \\
\hline 250 & $0 O B$ & V1 & 3.309 & 2.010 & 0.156 & 1.418 & 64 \\
\hline & & V2 & 3.229 & 1.744 & 0.144 & 1.321 & 36 \\
\hline & & v3 & -2.070 & 5.167 & 0.555 & 2.273 & 36 \\
\hline 251 & 12 & V1 & 5.314 & 64.207 & 0.699 & 8.013 & 121 \\
\hline 251 & $12 \mathrm{~B}$ & V1 & -1.316 & 14.339 & 0.900 & 3.787 & 100 \\
\hline & & V2 & -3.581 & 27.975 & 0.695 & 5.289 & 49 \\
\hline 252 & 00 & $\begin{array}{l}\mathrm{V} 1 \\
\mathrm{~V} 2\end{array}$ & 6.783 & 9.069 & 0.165 & 3.012 & 49 \\
\hline 252 & $00 B$ & $\begin{array}{l}\mathrm{V} 2 \\
\mathrm{~V} 1\end{array}$ & $\begin{array}{r}3.036 \\
-0.475\end{array}$ & 6.542 & 0.419 & 2.558 & 49 \\
\hline 253 & 00 & V1 & 0.421 & $\begin{array}{r}1.133 \\
33.841\end{array}$ & 0.854 & 1.065 & 36 \\
\hline & & V2 & -2.925 & 93.933 & 0.934 & $\begin{array}{l}5.817 \\
9.692\end{array}$ & 49 \\
\hline & & V3 & -1.183 & 5.605 & 0.814 & 2.368 & $\begin{array}{l}49 \\
49\end{array}$ \\
\hline & & V4 & -0.654 & 1.079 & 0.731 & 1.039 & 36 \\
\hline
\end{tabular}


Table 9: FNOC component wind statistics - 1-day averages

\begin{tabular}{|c|c|c|c|c|c|}
\hline DAY & $\frac{\text { MEAN }}{(\mathrm{m} / \mathrm{s})}$ & $\frac{\text { VAR }}{\left(\mathrm{m}^{2} / \mathrm{s}^{2}\right)}$ & WVAR & $\frac{\underline{\text { SDEV }}}{(\mathrm{m} / \mathrm{s})}$ & $\underline{\mathrm{N}}$ \\
\hline $224 \mathrm{U}$ & -0.234 & 20.735 & 1.000 & 4.554 & 378 \\
\hline V & 1.193 & 3.309 & 0.701 & 1.819 & 378 \\
\hline $225 \mathrm{U}$ & -1.229 & 23.873 & 0.943 & 4.886 & 340 \\
\hline V & 0.758 & 7.354 & 0.930 & 2.712 & 340 \\
\hline $226 \mathrm{U}$ & -2.223 & 18.383 & 0.789 & 4.288 & 463 \\
\hline V & -0.201 & 13.628 & 0.999 & 3.692 & 463 \\
\hline $227 \mathrm{U}$ & 0.624 & 35.262 & 0.991 & 5.938 & 499 \\
\hline V & -0.820 & 9.059 & 0.933 & 3.010 & 499 \\
\hline $228 \mathrm{U}$ & 2.234 & 31.682 & 0.866 & 5.629 & 453 \\
\hline V & -0.013 & 7.074 & 1.002 & 2.660 & 453 \\
\hline $229 \mathrm{U}$ & 0.807 & 21.933 & 0.973 & 4.683 & 631 \\
\hline V & 2.245 & 9.824 & 0.662 & 3.134 & 631 \\
\hline $230 \mathrm{U}$ & 0.222 & 20.806 & 0.999 & 4.561 & 695 \\
\hline V & 2.980 & 7.705 & 0.465 & 2.776 & 695 \\
\hline $231 \mathrm{U}$ & 3.772 & 6.800 & 0.330 & 2.608 & 134 \\
\hline V & 3.080 & 6.322 & 0.401 & 2.514 & 134 \\
\hline $232 \mathrm{U}$ & 1.556 & 18.062 & 0.884 & 4.250 & 466 \\
\hline V & 1.572 & 9.150 & 0.789 & 3.025 & 466 \\
\hline $233 \mathrm{U}$ & 1.190 & 16.892 & 0.924 & 4.110 & 636 \\
\hline V & 0.532 & 11.917 & 0.978 & 3.452 & 636 \\
\hline $234 \mathrm{U}$ & -0.568 & 14.678 & 0.980 & 3.831 & 623 \\
\hline V & 0.455 & 9.704 & 0.981 & 3.115 & 623 \\
\hline $235 \mathrm{U}$ & 0.696 & 20.454 & 0.978 & 4.523 & 662 \\
\hline V & 1.184 & 10.423 & 0.883 & 3.228 & 662 \\
\hline $236 \mathrm{U}$ & 1.452 & 19.291 & 0.903 & 4.392 & 693 \\
\hline V & 2.038 & 10.751 & 0.722 & 3.279 & 693 \\
\hline $237 \mathrm{U}$ & 0.582 & 10.881 & 0.974 & 3.299 & 206 \\
\hline V & 0.518 & 1.926 & 0.881 & 1.388 & 206 \\
\hline $238 \mathrm{U}$ & -1.388 & 18.064 & 0.905 & 4.250 & 545 \\
\hline V & 3.324 & 6.577 & 0.373 & 2.565 & 545 \\
\hline $239 \mathrm{U}$ & -1.528 & 20.189 & 0.899 & 4.493 & 372 \\
\hline V & 3.513 & 7.751 & 0.386 & 2.784 & 372 \\
\hline $240 \mathrm{U}$ & -2.285 & 8.209 & 0.612 & 2.865 & 412 \\
\hline V & 1.101 & 13.837 & 0.922 & 3.720 & 412 \\
\hline $241 \mathrm{U}$ & -2.497 & 5.200 & 0.455 & 2.280 & 453 \\
\hline $\mathrm{V}$ & 0.052 & 14.181 & 1.002 & 3.766 & 453 \\
\hline $242 \mathrm{U}$ & -1.982 & 6.738 & 0.633 & 2.596 & 260 \\
\hline V & -0.312 & 9.412 & 0.994 & 3.068 & 260 \\
\hline
\end{tabular}




$\begin{array}{rlllll}243 \mathrm{U} & -1.405 & 10.521 & 0.849 & 3.244 & 98 \\ \mathrm{~V} & 1.237 & 3.641 & 0.709 & 1.908 & 98 \\ 244 \mathrm{U} & -2.014 & 37.820 & 0.906 & 6.150 & 340 \\ \mathrm{~V} & 1.529 & 21.015 & 0.902 & 4.584 & 340 \\ 245 \mathrm{U} & -1.179 & 17.432 & 0.930 & 4.175 & 255 \\ \mathrm{~V} & 0.670 & 2.748 & 0.862 & 1.658 & 255 \\ 246 \mathrm{U} & -1.066 & 28.716 & 0.964 & 5.359 & 378 \\ \text { V } & 0.811 & 22.394 & 0.974 & 4.732 & 378 \\ 247 \mathrm{U} & 1.851 & 35.255 & 0.913 & 5.938 & 612 \\ \mathrm{~V} & 3.104 & 28.171 & 0.746 & 5.308 & 612 \\ 248 \mathrm{U} & 2.225 & 28.439 & 0.852 & 5.333 & 920 \\ \mathrm{~V} & 3.835 & 23.687 & 0.617 & 4.867 & 920 \\ 249 \mathrm{U} & -1.576 & 22.789 & 0.904 & 4.774 & 423 \\ \mathrm{~V} & 1.199 & 12.631 & 0.900 & 3.554 & 423 \\ 250 \mathrm{U} & -1.667 & 20.537 & 0.884 & 4.532 & 257 \\ \mathrm{~V} & 0.625 & 8.086 & 0.957 & 2.844 & 257 \\ 251 \mathrm{U} & 0.861 & 32.198 & 0.980 & 5.674 & 404 \\ \mathrm{~V} & 1.980 & 41.203 & 0.915 & 6.419 & 404 \\ 252 \mathrm{U} & -0.418 & 44.690 & 0.999 & 6.685 & 317 \\ \mathrm{~V} & 0.820 & 32.411 & 0.983 & 5.693 & 317\end{array}$


Table 10: FNOC component wind statistics 1 -day $/ 10^{\circ}$ latitude averages

$$
20^{\circ}-30^{\circ} \mathrm{N}
$$

\begin{tabular}{|c|c|c|c|c|c|}
\hline DAY & MEAN & $\underline{\text { VAR }}$ & WVAR & SDEV & $\underline{N}$ \\
\hline & $(\mathrm{m} / \mathrm{s})$ & $\left(\mathrm{m}^{2} / \mathrm{s}^{2}\right)$ & & $(\mathrm{m} / \mathrm{s})$ & \\
\hline $224 \mathrm{U}$ & -4.231 & 4.351 & 0.196 & 2.086 & 172 \\
\hline V & 0.040 & 0.941 & 1.004 & 0.970 & 172 \\
\hline $225 \mathrm{U}$ & -4.965 & 9.001 & 0.268 & 3.000 & 157 \\
\hline V & 0.384 & 7.313 & 0.986 & 2.704 & 157 \\
\hline $226 \mathrm{U}$ & -5.617 & 5.126 & 0.140 & 2.264 & 234 \\
\hline V & -2.169 & 6.332 & 0.575 & 2.516 & 234 \\
\hline $227 \mathrm{U}$ & -3.559 & 10.310 & 0.450 & 3.211 & 257 \\
\hline V & -1.502 & 7.547 & 0.772 & 2.747 & 257 \\
\hline $228 \mathrm{U}$ & -1.197 & 14.172 & 0.911 & 3.765 & 255 \\
\hline V & 0.258 & 3.090 & 0.983 & 1.758 & 255 \\
\hline $229 \mathrm{U}$ & -2.290 & 11.989 & 0.698 & 3.462 & 255 \\
\hline V & 0.783 & 3.943 & 0.868 & 1.986 & 255 \\
\hline $230 \mathrm{U}$ & -2.846 & 14.322 & 0.640 & 3.785 & 291 \\
\hline $\mathrm{V}$ & 1.306 & 4.004 & 0.703 & 2.001 & 291 \\
\hline $231 \mathrm{U}$ & none & & & & \\
\hline V & none & & & & \\
\hline $232 \mathrm{U}$ & -3.658 & 3.864 & 0.224 & 1.966 & 134 \\
\hline $\mathrm{V}$ & 1.331 & 3.192 & 0.646 & 1.787 & 134 \\
\hline $233 \mathrm{U}$ & -2.869 & 6.129 & 0.428 & 2.476 & 183 \\
\hline V & 1.016 & 2.349 & 0.697 & 1.533 & 183 \\
\hline $234 \mathrm{U}$ & -1.812 & 8.910 & 0.733 & 2.985 & 268 \\
\hline V & 0.421 & 3.927 & 0.960 & 1.982 & 268 \\
\hline $235 \mathrm{U}$ & -1.686 & 5.977 & 0.680 & 2.445 & 234 \\
\hline V & 0.302 & 4.197 & 0.983 & 2.049 & 234 \\
\hline $236 \mathrm{U}$ & -0.257 & 4.509 & 0.989 & 2.123 & 298 \\
\hline V & 0.550 & 2.169 & 0.880 & 1.473 & 298 \\
\hline $237 \mathrm{U}$ & -0.508 & 6.168 & 0.965 & 2.484 & 170 \\
\hline $\mathrm{V}$ & 0.393 & 1.603 & 0.917 & 1.266 & 170 \\
\hline $238 \mathrm{U}$ & -1.276 & 8.599 & 0.844 & 2.932 & 234 \\
\hline $\mathrm{V}$ & 2.004 & 5.945 & 0.598 & 2.438 & 234 \\
\hline $239 \mathrm{U}$ & -2.051 & 7.415 & 0.640 & 2.723 & 206 \\
\hline V & 2.164 & 4.995 & 0.517 & 2.235 & 206 \\
\hline $240 \mathrm{U}$ & -3.048 & 5.489 & 0.372 & 2.343 & 327 \\
\hline V & -0.115 & 6.111 & 1.001 & 2.472 & 327 \\
\hline
\end{tabular}




$\begin{array}{rlllll}241 \mathrm{U} & -2.527 & 6.267 & 0.496 & 2.503 & 291 \\ \mathrm{~V} & -0.125 & 7.006 & 1.001 & 2.647 & 291 \\ 242 \mathrm{U} & -2.040 & 6.986 & 0.631 & 2.643 & 98 \\ \mathrm{~V} & 0.762 & 5.520 & 0.913 & 2.349 & 98 \\ 243 \mathrm{U} & -3.768 & 3.037 & 0.177 & 1.743 & 49 \\ \mathrm{~V} & 0.470 & 2.489 & 0.936 & 1.578 & 49 \\ 244 \mathrm{U} & -3.822 & 23.897 & 0.624 & 4.888 & 134 \\ \mathrm{~V} & 0.766 & 10.227 & 0.952 & 3.198 & 134 \\ 245 \mathrm{U} & -4.173 & 8.871 & 0.338 & 2.978 & 134 \\ \mathrm{~V} & 0.120 & 1.395 & 0.997 & 1.181 & 134 \\ 246 \mathrm{U} & -2.184 & 11.917 & 0.716 & 3.452 & 306 \\ \mathrm{~V} & 0.038 & 9.739 & 1.003 & 3.121 & 306 \\ 247 \mathrm{U} & -1.808 & 12.309 & 0.792 & 3.508 & 342 \\ \mathrm{~V} & 0.631 & 10.371 & 0.966 & 3.220 & 342 \\ 248 \mathrm{U} & -2.533 & 9.352 & 0.594 & 3.058 & 359 \\ \mathrm{~V} & 1.711 & 6.788 & 0.700 & 2.605 & 359 \\ 249 \mathrm{U} & -3.876 & 8.897 & 0.372 & 2.983 & 310 \\ \mathrm{~V} & 0.073 & 9.963 & 1.003 & 3.156 & 310 \\ 250 \mathrm{U} & -3.837 & 7.468 & 0.337 & 2.733 & 193 \\ \mathrm{~V} & -0.265 & 6.925 & 0.995 & 2.631 & 193 \\ 251 \mathrm{U} & -2.951 & 11.617 & 0.573 & 3.408 & 185 \\ \mathrm{~V} & 0.000 & 13.077 & 1.005 & 3.616 & 185 \\ 252 \mathrm{U} & -2.127 & 4.899 & 0.522 & 2.213 & 121 \\ \mathrm{~V} & 0.893 & 6.416 & 0.896 & 2.533 & 121\end{array}$

$30^{\circ}-40^{\circ} \mathrm{N}$

\begin{tabular}{|c|c|c|c|c|c|}
\hline DAY & $\frac{\text { MEAN }}{(\mathrm{m} / \mathrm{s})}$ & $\frac{\mathrm{VAR}}{\left(\mathrm{m}^{2} / \mathrm{s}^{2}\right)}$ & WVAR & $\frac{\text { SDEV }}{(m / s)}$ & $\underline{N}$ \\
\hline $224 \mathrm{U}$ & 1.983 & 4.258 & 0.522 & 2.064 & 170 \\
\hline V & 2.603 & 2.689 & 0.285 & 1.640 & 170 \\
\hline $225 \mathrm{U}$ & 0.012 & 7.347 & 1.005 & 2.711 & 183 \\
\hline V & 1.952 & 9.338 & 0.713 & 3.056 & 183 \\
\hline $226 \mathrm{U}$ & 1.612 & 8.357 & 0.766 & 2.891 & 193 \\
\hline V & 0.620 & 6.465 & 0.948 & 2.543 & 193 \\
\hline $227 \mathrm{U}$ & 3.176 & 10.288 & 0.506 & 3.208 & 193 \\
\hline V & 0.822 & 2.001 & 0.751 & 1.415 & 193 \\
\hline $228 \mathrm{U}$ & 3.955 & 9.536 & 0.379 & 3.088 & 198 \\
\hline V & 1.010 & 3.040 & 0.751 & 1.744 & 198 \\
\hline $229 \mathrm{U}$ & 1.332 & 3.615 & 0.673 & 1.901 & 219 \\
\hline V & 4.576 & 7.221 & 0.257 & 2.687 & 219 \\
\hline $230 \mathrm{U}$ & 0.842 & 4.473 & 0.867 & 2.115 & 219 \\
\hline V & 4.630 & 6.054 & 0.220 & 2.460 & 219 \\
\hline
\end{tabular}




\begin{tabular}{|c|c|c|c|c|c|}
\hline $231 \mathrm{U}$ & 3.972 & 1.600 & 0.092 & 1.265 & 85 \\
\hline V & 1.802 & 4.250 & 0.571 & 2.062 & 85 \\
\hline $232 \mathrm{U}$ & 3.878 & 7.883 & 0.344 & 2.808 & 268 \\
\hline V & 2.441 & 5.469 & 0.480 & 2.339 & 268 \\
\hline $233 \mathrm{U}$ & 2.926 & 13.020 & 0.604 & 3.608 & 353 \\
\hline V & 0.832 & 12.956 & 0.952 & 3.599 & 353 \\
\hline $234 \mathrm{U}$ & 1.240 & 12.151 & 0.891 & 3.486 & 270 \\
\hline V & -0.047 & 13.226 & 1.004 & 3.637 & 270 \\
\hline $235 \mathrm{U}$ & 2.884 & 11.934 & 0.590 & 3.455 & 294 \\
\hline V & 0.992 & 8.860 & 0.903 & 2.977 & 294 \\
\hline $236 \mathrm{U}$ & 2.196 & 12.133 & 0.717 & 3.483 & 338 \\
\hline V & 0.808 & 2.265 & 0.778 & 1.505 & 338 \\
\hline $237 \mathrm{U}$ & 1.117 & 9.936 & 0.893 & 3.152 & 157 \\
\hline V & 0.541 & 1.628 & 0.852 & 1.276 & 157 \\
\hline $238 \mathrm{U}$ & 0.487 & 16.981 & 0.990 & 4.121 & 279 \\
\hline V & 3.237 & 5.958 & 0.363 & 2.441 & 279 \\
\hline $239 \mathrm{U}$ & 4.160 & 6.329 & 0.269 & 2.516 & 85 \\
\hline V & 5.107 & 5.581 & 0.177 & 2.362 & 85 \\
\hline $240 \mathrm{U}$ & 0.650 & 7.886 & 0.960 & 2.808 & 85 \\
\hline V & 5.780 & 16.076 & 0.326 & 4.009 & 85 \\
\hline $241 \mathrm{U}$ & -1.866 & 3.434 & 0.499 & 1.853 & 98 \\
\hline V & 3.079 & 21.397 & 0.698 & 4.626 & 98 \\
\hline $242 \mathrm{U}$ & -1.046 & 7.724 & 0.884 & 2.779 & 98 \\
\hline V & 0.880 & 4.370 & 0.857 & 2.090 & 98 \\
\hline $243 \mathrm{U}$ & 0.957 & 6.831 & 0.898 & 2.614 & 49 \\
\hline V & 2.004 & 3.668 & 0.482 & 1.915 & 49 \\
\hline $244 \mathrm{U}$ & -2.504 & 66.851 & 0.921 & 8.176 & 121 \\
\hline$V$ & 2.102 & 44.686 & 0.917 & 6.685 & 121 \\
\hline $245 \mathrm{U}$ & 2.540 & 8.995 & 0.587 & 2.999 & 72 \\
\hline V & 1.078 & 2.387 & 0.679 & 1.545 & 72 \\
\hline $246 \mathrm{U}$ & 3.684 & 73.020 & 0.853 & 8.545 & 72 \\
\hline$V$ & 4.097 & 63.553 & 0.800 & 7.972 & 72 \\
\hline $247 \mathrm{C}$ & 4.119 & 40.899 & 0.710 & 6.395 & 149 \\
\hline $\mathrm{V}$ & 3.739 & 42.750 & 0.757 & 6.538 & 149 \\
\hline $248 \mathrm{C}$ & 3.605 & 10.653 & 0.451 & 3.264 & 355 \\
\hline V & 2.616 & 17.674 & 0.722 & 4.204 & 355 \\
\hline $249 \mathrm{U}$ & 3.200 & 12.635 & 0.554 & 3.555 & 149 \\
\hline V & 3.738 & 7.866 & 0.361 & 2.805 & 149 \\
\hline $250 \mathrm{U}$ & 4.874 & 2.812 & 0.106 & 1.677 & 64 \\
\hline$V$ & 3.309 & 2.010 & 0.156 & 1.418 & 64 \\
\hline $251 \mathrm{U}$ & 2.511 & 13.718 & 0.690 & 3.704 & 98 \\
\hline V & 1.601 & 45.461 & 0.956 & 6.742 & 98 \\
\hline $252 \mathrm{U}$ & 4.344 & 27.428 & 0.595 & 5.237 & 147 \\
\hline V & 0.892 & 53.689 & 0.992 & 7.327 & 147 \\
\hline
\end{tabular}




\begin{tabular}{|c|c|c|c|c|c|}
\hline DAY & $\frac{\text { MEAN }}{(\mathrm{m} / \mathrm{s})}$ & $\frac{\text { VAR }}{\left(m^{2} / s^{2}\right)}$ & WVAR & $\frac{\mathrm{SDEV}}{(\mathrm{m} / \mathrm{s})}$ & $\underline{N}$ \\
\hline $224 \mathrm{U}$ & 8.389 & 2.700 & 0.037 & 1.643 & 36 \\
\hline V & 0.041 & 0.505 & 1.025 & 0.710 & 36 \\
\hline $225 \mathrm{U}$ & none & & & & \\
\hline V & none & & & & \\
\hline $226 \mathrm{U}$ & -0.724 & 2.306 & 0.834 & 1.518 & 36 \\
\hline V & 8.182 & 0.394 & 0.006 & 0.627 & 36 \\
\hline $227 \mathrm{U}$ & 12.509 & 5.660 & 0.035 & 2.379 & 49 \\
\hline V & -3.708 & 23.905 & 0.643 & 4.889 & 49 \\
\hline $228 \mathrm{U}$ & 12.509 & 5.660 & 0.035 & 2.379 & 49 \\
\hline V & -3.708 & 23.905 & 0.643 & 4.889 & 49 \\
\hline $229 \mathrm{U}$ & 5.107 & 29.329 & 0.531 & 5.416 & 157 \\
\hline V & 1.370 & 11.273 & 0.862 & 3.357 & 157 \\
\hline $230 \mathrm{U}$ & 3.596 & 17.847 & 0.581 & 4.225 & 234 \\
\hline V & 3.593 & 6.897 & 0.349 & 2.626 & 234 \\
\hline $231 \mathrm{U}$ & 3.290 & 15.740 & 0.600 & 3.967 & 49 \\
\hline V & 5.297 & 2.169 & 0.072 & 1.473 & 49 \\
\hline $232 \mathrm{U}$ & 2.305 & 9.951 & 0.656 & 3.155 & 100 \\
\hline V & -0.365 & 20.398 & 1.003 & 4.516 & 100 \\
\hline $233 \mathrm{U}$ & 1.295 & 15.063 & 0.905 & 3.881 & 172 \\
\hline V & 0.463 & 20.688 & 0.995 & 4.548 & 172 \\
\hline $234 \mathrm{U}$ & -2.345 & 22.947 & 0.812 & 4.790 & 121 \\
\hline V & 2.819 & 12.111 & 0.607 & 3.480 & 121 \\
\hline $235 \mathrm{U}$ & 0.979 & 33.752 & 0.976 & 5.810 & 234 \\
\hline V & 2.040 & 19.962 & 0.830 & 4.468 & 234 \\
\hline $236 \mathrm{U}$ & 1.312 & 33.119 & 0.954 & 5.755 & 242 \\
\hline V & 4.166 & 19.467 & 0.530 & 4.412 & 242 \\
\hline $237 \mathrm{U}$ & none & & & & \\
\hline V & none & & & & \\
\hline $238 \mathrm{U}$ & -5.181 & 10.639 & 0.284 & 3.262 & 162 \\
\hline V & 4.515 & 5.279 & 0.206 & 2.298 & 162 \\
\hline $239 \mathrm{U}$ & -6.168 & 11.113 & 0.227 & 3.334 & 81 \\
\hline V & 5.269 & 6.788 & 0.197 & 2.605 & 81 \\
\hline $240 \mathrm{U}$ & none & & & & \\
\hline V & none & & & & \\
\hline $241 \mathrm{U}$ & -3.205 & 1.310 & 0.113 & 1.145 & 113 \\
\hline V & -2.247 & 8.278 & 0.625 & 2.877 & 113 \\
\hline $242 \mathrm{U}$ & -3.205 & 1.310 & 0.113 & 1.145 & 113 \\
\hline V & -2.247 & 8.278 & 0.625 & 1.145 & 113 \\
\hline $243 \mathrm{U}$ & none & & & & \\
\hline
\end{tabular}




$\begin{array}{rlllll}\text { V } & \text { none } & & & & \\ 244 \mathrm{U} & 1.536 & 0.987 & 0.296 & 0.993 & 85 \\ \mathrm{~V} & 1.914 & 3.231 & 0.471 & 1.797 & 85 \\ 245 \mathrm{U} & 1.546 & 1.006 & 0.298 & 1.003 & 49 \\ \mathrm{~V} & 1.575 & 5.215 & 0.687 & 2.284 & 49 \\ 246 \mathrm{U} & \text { none } & & & & \\ \text { V } & \text { none } & & & & \\ 247 \mathrm{U} & 6.185 & 1.376 & 0.035 & 1.173 & 121 \\ \mathrm{~V} & 9.310 & 4.477 & 0.049 & 2.116 & 121 \\ 248 \mathrm{U} & 6.687 & 24.754 & 0.357 & 4.975 & 242 \\ \text { V } & 8.504 & 24.988 & 0.257 & 4.999 & 242 \\ 249 \mathrm{U} & \text { none } & & & & \\ \text { V } & \text { none } & & & & \\ 250 \mathrm{U} & \text { none } & & & & \\ \text { V } & \text { none } & & & & \\ 251 \mathrm{U} & 5.354 & 34.252 & 0.547 & 5.852 & 121 \\ \text { V } & 5.314 & 64.207 & 0.699 & 8.013 & 121 \\ 252 \mathrm{U} & -10.485 & 18.268 & 0.143 & 4.274 & 49 \\ \text { V } & 0.421 & 33.841 & 1.015 & 5.817 & 49\end{array}$


Table 11: FNOC wind statistics - 1-day vector average plus direction bins (wind blowing toward a given direction)

\begin{tabular}{|c|c|c|c|c|c|c|c|c|c|c|}
\hline DAY & MEAN & VARSPD & SDSPD & VARVEC & SDVEC & $\mathbf{N}$ & NORTH & EAST & SOUTH & WEST \\
\hline 224 & 4.595 & 4.359 & 2.088 & 24.043 & 4.903 & 378 & 0.29 & 0.25 & 0.00 & 0.46 \\
\hline 225 & 5.323 & 4.898 & 2.213 & 31.225 & 5.588 & 340 & 0.31 & 0.17 & 0.08 & 0.44 \\
\hline 226 & 5.619 & 5.360 & 2.315 & 32.011 & 5.658 & 463 & 0.17 & 0.23 & 0.17 & 0.43 \\
\hline 227 & 5.583 & 14.152 & 3.762 & 44.320 & 6.657 & 499 & 0.15 & 0.39 & 0.11 & 0.35 \\
\hline 228 & 5.393 & 14.605 & 3.822 & 38.754 & 6.225 & 453 & 0.16 & 0.55 & 0.04 & 0.26 \\
\hline 229 & 5.421 & 8.026 & 2.833 & 31.756 & 5.635 & 631 & 0.47 & 0.26 & 0.03 & 0.24 \\
\hline 230 & 5.553 & 6.569 & 2.563 & 28.510 & 5.339 & 695 & 0.53 & 0.19 & 0.01 & 0.27 \\
\hline 231 & 5.659 & 4.378 & 2.092 & 13.121 & 3.622 & 134 & 0.26 & 0.72 & 0.01 & 0.00 \\
\hline 232 & 5.178 & 5.241 & 2.289 & 27.210 & 5.216 & 466 & 0.25 & 0.45 & 0.06 & 0.24 \\
\hline 233 & 5.003 & 5.447 & 2.334 & 28.808 & 5.367 & 636 & 0.23 & 0.39 & 0.14 & 0.24 \\
\hline 234 & 4.458 & 5.006 & 2.237 & 24.381 & 4.938 & 623 & 0.23 & 0.26 & 0.14 & 0.37 \\
\hline 235 & 5.077 & 6.956 & 2.637 & 30.875 & 5.557 & 662 & 0.32 & 0.32 & 0.09 & 0.27 \\
\hline 236 & 5.018 & 11.093 & 3.331 & 30.041 & 5.481 & 693 & 0.37 & 0.42 & 0.04 & 0.18 \\
\hline 237 & 3.024 & 4.227 & 2.056 & 12.807 & 3.579 & 206 & 0.26 & 0.44 & 0.02 & 0.28 \\
\hline 238 & 5.627 & 5.921 & 2.433 & 24.640 & 4.964 & 545 & 0.50 & 0.13 & 0.02 & 0.35 \\
\hline 239 & 5.916 & 7.566 & 2.751 & 27.940 & 5.286 & 372 & 0.50 & 0.08 & 0.01 & 0.41 \\
\hline 240 & 4.710 & 6.256 & 2.501 & 22.045 & 4.695 & 412 & 0.30 & 0.03 & 0.08 & 0.59 \\
\hline 241 & 4.512 & 5.232 & 2.287 & 19.379 & 4.402 & 453 & 0.23 & 0.04 & 0.17 & 0.56 \\
\hline 242 & 4.144 & 2.946 & 1.716 & 16.149 & 4.019 & 260 & 0.23 & 0.09 & 0.18 & 0.51 \\
\hline 243 & 3.943 & 1.997 & 1.413 & 14.163 & 3.763 & 98 & 0.31 & 0.14 & 0.01 & 0.54 \\
\hline 244 & 6.038 & 28.676 & 5.355 & 58.834 & 7.670 & 340 & 0.34 & 0.21 & 0.02 & 0.42 \\
\hline 245 & 4.080 & 5.313 & 2.305 & 20.179 & 4.492 & 255 & 0.23 & 0.29 & 0.03 & 0.45 \\
\hline 246 & 5.754 & 19.715 & 4.440 & 51.109 & 7.149 & 378 & 0.28 & 0.17 & 0.12 & 0.43 \\
\hline 247 & 7.007 & 19.227 & 4.385 & 57.280 & 7.568 & 612 & 0.46 & 0.17 & 0.09 & 0.28 \\
\hline 248 & 7.180 & 20.204 & 4.495 & 52.124 & 7.220 & 920 & 0.48 & 0.26 & 0.05 & 0.21 \\
\hline 249 & 5.583 & 8.102 & 2.846 & 35.419 & 5.951 & 423 & 0.27 & 0.17 & 0.05 & 0.50 \\
\hline 250 & 5.230 & 4.344 & 2.084 & 28.622 & 5.350 & 257 & 0.20 & 0.23 & 0.03 & 0.54 \\
\hline 251 & 7.382 & 23.454 & 4.843 & 73.400 & 8.567 & 404 & 0.35 & 0.19 & 0.22 & 0.24 \\
\hline 252 & 7.403 & 22.970 & 4.793 & 77.100 & 8.781 & 317 & 0.30 & 0.20 & 0.13 & 0.38 \\
\hline
\end{tabular}


Table 12: FNOC wind statistics - 3-day average plus direction bins

(wind blowing toward a given direction)

$\begin{array}{lrrrrrrrrrr}\text { DAYS } & \text { MEAN } & \text { VARSPD } & \text { SDSPD } & \text { VARVEC } & \text { SDVEC } & \text { N } & \text { NORTH } & \text { EAST } & \text { SOUTH } & \text { WEST } \\ 224-226 & 5.184 & 4.989 & 2.234 & 29.464 & 5.428 & 962 & 0.27 & 0.21 & 0.08 & 0.44 \\ 227-229 & 5.355 & 9.885 & 3.144 & 37.231 & 6.102 & 1279 & 0.31 & 0.35 & 0.06 & 0.28 \\ 230-232 & 5.402 & 6.072 & 2.464 & 28.868 & 5.373 & 1161 & 0.42 & 0.29 & 0.03 & 0.26 \\ 233-235 & 4.923 & 5.981 & 2.446 & 29.025 & 5.387 & 1455 & 0.28 & 0.34 & 0.11 & 0.28 \\ 236-238 & 5.286 & 8.907 & 2.985 & 30.038 & 5.481 & 1238 & 0.42 & 0.29 & 0.03 & 0.25 \\ 239-241 & 5.145 & 6.766 & 2.601 & 26.412 & 5.139 & 825 & 0.35 & 0.06 & 0.10 & 0.49 \\ 242-244 & 5.218 & 18.385 & 4.288 & 41.112 & 6.412 & 600 & 0.29 & 0.16 & 0.09 & 0.46 \\ 245-247 & 6.146 & 16.910 & 4.112 & 48.754 & 6.982 & 867 & 0.39 & 0.21 & 0.07 & 0.33 \\ 248-250 & 6.754 & 17.383 & 4.169 & 51.313 & 7.163 & 1177 & 0.42 & 0.25 & 0.04 & 0.29 \\ 250-252 & 7.047 & 19.952 & 4.467 & 68.832 & 8.297 & 844 & 0.26 & 0.23 & 0.15 & 0.36\end{array}$


Table 13: Boxed mean differences - 18 Aug 1978 (Day 224)

Mean Atlas SASS winds - $10 \mathrm{deg} 1 \mathrm{at} / 10 \mathrm{deg}$ lon boxes and total for data from revs $744,750,751,758$

LON1 LON2 LAT1 LAT2 UMEAN UVAR VMEAN VVAR N

$\begin{array}{rrrrrrrrr}60 & 70 & 40 & 50 & 2.33 & 6.39 & 5.59 & 0.90 & 16 \\ 50 & 60 & 40 & 50 & 2.35 & 6.97 & 4.21 & 4.78 & 51 \\ 40 & 50 & 40 & 50 & 3.05 & 7.59 & 1.51 & 9.80 & 73 \\ 70 & 80 & 30 & 40 & 5.23 & 3.47 & 1.22 & 4.88 & 21 \\ 60 & 70 & 30 & 40 & 2.82 & 8.53 & 5.73 & 8.33 & 89 \\ 50 & 60 & 30 & 40 & 1.40 & 6.21 & 1.28 & 6.53 & 94 \\ 40 & 50 & 30 & 40 & -0.67 & 10.75 & 0.59 & 6.68 & 81 \\ 70 & 80 & 20 & 30 & -4.44 & 23.36 & 1.00 & 5.68 & 49 \\ 60 & 70 & 20 & 30 & -4.54 & 11.62 & 2.34 & 4.30 & 116 \\ 50 & 60 & 20 & 30 & -1.37 & 16.07 & -0.54 & 4.41 & 22 \\ 40 & 50 & 20 & 30 & -3.93 & 18.90 & -0.65 & 2.96 & 64 \\ 40 & 80 & 20 & 50 & -0.31 & 20.89 & 2.05 & 9.72 & 676\end{array}$

Mean ENOC winds - $10 \mathrm{deg}$ lat/10 deg lon boxes and total for 12218 Aug

$\begin{array}{rrrrrrrrr}\text { LON1 } & \text { LON2 } & \text { LAT1 } & \text { LAT2 } & \text { UMEAN } & \text { UVAR } & \text { VMEAN } & \text { VVAR } & \text { N } \\ 70 & 80 & 40 & 50 & 3.42 & 10.72 & -2.86 & 23.50 & 121 \\ 60 & 70 & 40 & 50 & 5.42 & 4.12 & 0.26 & 29.43 & 121 \\ 50 & 60 & 40 & 50 & 4.64 & 6.35 & 7.04 & 2.61 & 121 \\ 40 & 50 & 40 & 50 & 5.37 & 7.55 & 1.18 & 14.05 & 121 \\ 70 & 80 & 30 & 40 & 1.64 & 4.99 & 0.03 & 4.73 & 121 \\ 60 & 70 & 30 & 40 & 2.19 & 0.20 & 4.79 & 4.00 & 121 \\ 50 & 60 & 30 & 40 & 1.44 & 0.66 & 2.84 & 2.03 & 121 \\ 40 & 50 & 30 & 40 & 0.75 & 1.73 & 1.64 & 0.42 & 121 \\ 70 & 80 & 20 & 30 & -2.45 & 14.97 & 1.51 & 2.85 & 121 \\ 60 & 70 & 20 & 30 & -2.75 & 10.71 & 2.14 & 3.24 & 121 \\ 50 & 60 & 20 & 30 & -3.20 & 10.27 & 0.28 & 1.49 & 121 \\ 40 & 50 & 20 & 30 & -3.86 & 10.68 & -0.01 & 1.53 & 121 \\ 40 & 80 & 20 & 50 & 0.98 & 18.54 & 1.47 & 13.39 & 1271\end{array}$

Difference of boxed component means:

Atlas-ENOC: U component/V component

$\begin{array}{cccccc}* 50 * & \text { N/A } & -3.09 / 5.34 & -2.29 /-2.83 & -2.31 / 0.33 \\ \star 40 * & 3.59 / 1.19 & 0.63 / 0.93 & -0.04 /-1.55 & -1.42 /-1.05 \\ \star 30 * & -1.99 /-0.51 & -1.79 / 0.19 & 1.83 /-0.82 & -0.07 /-0.64 \\ \star 20 * & & & & & \end{array}$


Table 14: Boxed mean differences - 24 Aug 1978 (Day 230)

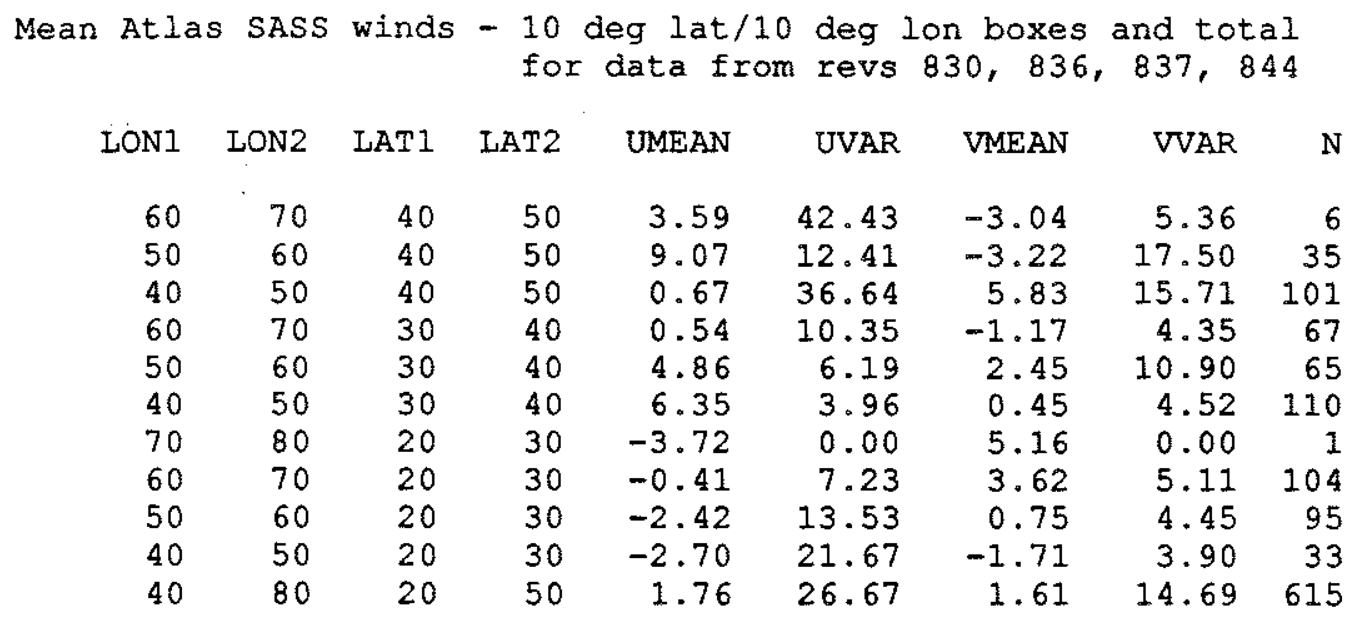

Mean FNOC winds - $10 \mathrm{deg}$ lat/10 deg lon boxes and total for 12224 Aug

$\begin{array}{rrrrrrrrr}\text { LON1 } & \text { LON2 } & \text { LAT1 } & \text { LAT2 } & \text { UMEAN } & \text { UVAR } & \text { VMEAN } & \text { VVAR } & \text { N } \\ 70 & 80 & 40 & 50 & -2.96 & 25.33 & 0.26 & 10.47 & 121 \\ 60 & 70 & 40 & 50 & 0.82 & 28.59 & -3.60 & 36.39 & 121 \\ 50 & 60 & 40 & 50 & 7.76 & 7.66 & -4.07 & 23.69 & 121 \\ 40 & 50 & 40 & 50 & 1.90 & 32.37 & 6.07 & 11.97 & 121 \\ 70 & 80 & 30 & 40 & 0.14 & 9.01 & -1.18 & 3.23 & 121 \\ 60 & 70 & 30 & 40 & 0.42 & 4.47 & -1.29 & 3.23 & 121 \\ 50 & 60 & 30 & 40 & 3.53 & 10.22 & 1.14 & 2.80 & 121 \\ 40 & 50 & 30 & 40 & 6.19 & 5.02 & 1.92 & 0.47 & 121 \\ 70 & 80 & 20 & 30 & -2.99 & 1.75 & 0.39 & 2.95 & 121 \\ 60 & 70 & 20 & 30 & -0.67 & 2.43 & 0.64 & 0.72 & 121 \\ 50 & 60 & 20 & 30 & -1.68 & 4.27 & 1.11 & 2.14 & 121 \\ 40 & 50 & 20 & 30 & -3.02 & 7.91 & 1.01 & 0.74 & 121 \\ 40 & 80 & 20 & 50 & 0.64 & 23.57 & 0.27 & 15.15 & 1271\end{array}$

Difference of boxed component means:

Atlas-FNOC: U component/V component

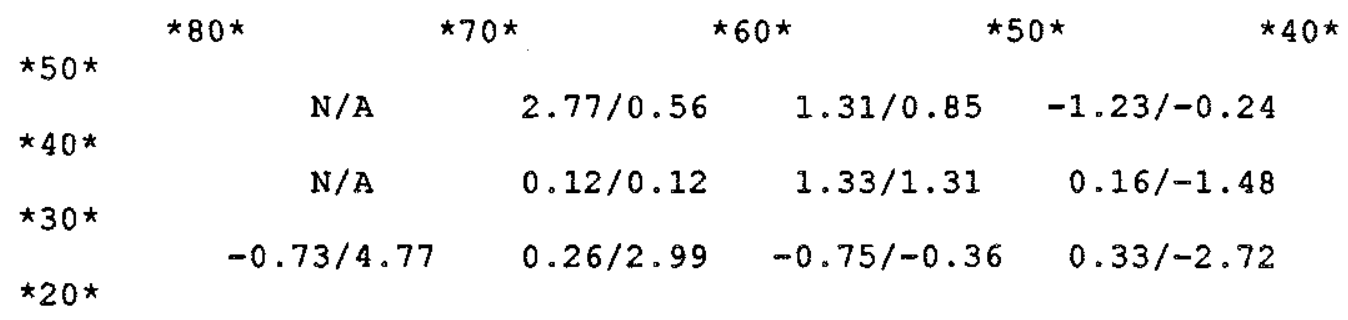


Table 15: Boxed mean differences - 5 Sep 1978 (Day 248)

Mean At las SASS winds - $10 \mathrm{deg}$ lat/10 deg lon boxes and total for data from revs $1002,1003,1008,1009,1016$

LON1 LON2 LAT1 LAT2 UMEAN UVAR VMEAN VVAR $N$

$\begin{array}{rrrrrrrrr}60 & 70 & 40 & 50 & 6.40 & 3.21 & 0.34 & 1.85 & 11 \\ 40 & 50 & 40 & 50 & 8.66 & 39.68 & 10.48 & 26.85 & 104 \\ 70 & 80 & 30 & 40 & -1.81 & 2.37 & -4.75 & 6.49 & 17 \\ 60 & 70 & 30 & 40 & 4.66 & 4.43 & -0.42 & 13.64 & 54 \\ 50 & 60 & 30 & 40 & 3.92 & 7.16 & 6.31 & 7.30 & 34 \\ 40 & 50 & 30 & 40 & 4.50 & 4.55 & 7.07 & 8.25 & 100 \\ 70 & 80 & 20 & 30 & -4.37 & 4.28 & 4.64 & 0.44 & 8 \\ 60 & 70 & 20 & 30 & -2.03 & 10.86 & 2.49 & 3.16 & 103 \\ 50 & 60 & 20 & 30 & -5.59 & 8.93 & -0.53 & 13.91 & 128 \\ 40 & 50 & 20 & 30 & -8.41 & 14.95 & 3.66 & 6.41 & 53 \\ 40 & 80 & 20 & 50 & 0.59 & 46.23 & 3.80 & 29.29 & 611\end{array}$

Mean FNOC winds - $10 \mathrm{deg} 1 \mathrm{lat} / 10 \mathrm{deg} 10 \mathrm{n}$ boxes and total for $12 \mathrm{z}$ sep

$\begin{array}{rrrrrrrrr}\text { LON1 } & \text { LON2 } & \text { LAT1 } & \text { LAT2 } & \text { UMEAN } & \text { UVAR } & \text { VMEAN } & \text { VNAR } & \text { N } \\ 70 & 80 & 40 & 50 & 1.42 & 19.44 & -2.58 & 25.93 & 121 \\ 60 & 70 & 40 & 50 & 4.84 & 14.65 & -2.11 & 16.94 & 121 \\ 50 & 60 & 40 & 50 & 4.62 & 24.93 & -2.10 & 21.69 & 121 \\ 40 & 50 & 40 & 50 & 7.19 & 47.83 & 7.70 & 44.39 & 121 \\ 70 & 80 & 30 & 40 & -1.06 & 6.70 & -0.97 & 6.11 & 121 \\ 60 & 70 & 30 & 40 & 3.28 & 3.00 & -0.10 & 5.53 & 121 \\ 50 & 60 & 30 & 40 & 6.58 & 6.00 & 3.65 & 8.63 & 121 \\ 40 & 50 & 30 & 40 & 5.60 & 15.97 & 8.51 & 4.66 & 121 \\ 70 & 80 & 20 & 30 & -1.58 & 0.42 & 1.23 & 0.90 & 121 \\ 60 & 70 & 20 & 30 & -1.94 & 3.15 & 2.70 & 0.73 & 121 \\ 50 & 60 & 20 & 30 & -2.42 & 7.10 & 1.58 & 4.11 & 121 \\ 40 & 50 & 20 & 30 & -5.49 & 10.55 & 1.91 & 4.92 & 121 \\ 40 & 80 & 20 & 50 & 1.63 & 29.98 & 1.65 & 23.98 & 1271\end{array}$

Difference of boxed component means:

At las-FNOC: $U$ component/V component

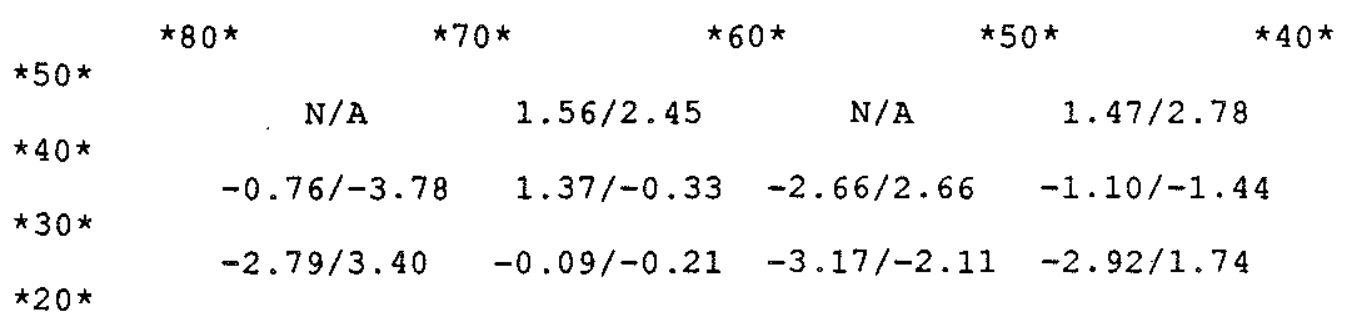


Table 16: Atlas-FNOC difference field component statistics - area averages (including mean squared difference)

\begin{tabular}{|c|c|c|c|c|c|c|c|c|c|c|c|c|}
\hline REI & & TIME & & & MEAN & VAR & WGTVAR & SDEV & DEVMN & MSD & WGTMSD & \\
\hline 658 & U1 & 224 & 000 & UI & 1.553 & 6.411 & 0.737 & 2.532 & 0.131 & 8.693 & 0.432 & \\
\hline 658 & v1 & 224 & $00 \mathrm{~V}$ & V1 & 0.039 & 6.407 & 1.021 & 2.531 & 0.131 & 6.277 & 0.567 & \\
\hline 658 & U2 & 224 & 00 & $\mathrm{U} 2$ & -0.697 & 1.610 & 0.785 & 1.269 & 0.045 & 2.051 & 0.088 & \\
\hline 658 & v2 & 224 & 00 & $\mathrm{~V} 2$ & 0.609 & 1.011 & 0.747 & 1.005 & 0.028 & 1.354 & 0.999 & \\
\hline 664 & $\mathrm{U} 1$ & 224 & $12 \mathrm{U}$ & $\mathrm{U1}$ & 0.187 & 1.971 & 1.010 & 1.404 & 0.055 & 1.951 & 0.036 & \\
\hline 664 & V1 & 224 & 12 & $\mathrm{~V} 1$ & 1.659 & 5.658 & 0.686 & 2.379 & 0.157 & 8.253 & 1.109 & \\
\hline 664 & $\mathrm{U} 2$ & 224 & 12 & $\mathrm{U} 2$ & -1.267 & 2.422 & 0.607 & 1.556 & 0.038 & 3.989 & 0.223 & \\
\hline 664 & V2 & 224 & $12 \mathrm{~V}$ & $\mathrm{~V} 2$ & -0.271 & 0.722 & 0.920 & 0.850 & 0.011 & 0.784 & 0.859 & \\
\hline 664 & U3 & 224 & 120 & U3 & 2.096 & 5.754 & 0.576 & 2.399 & 0.160 & 9.986 & 0.220 & \\
\hline 664 & V3 & 224 & $12 \mathrm{~V}$ & V3 & 3.744 & 14.367 & 0.513 & 3.790 & 0.399 & 27.984 & 0.610 & \\
\hline 665 & $\mathrm{U} 1$ & 224 & $12 \mathrm{~B}$ & U1 & -0.141 & 2.540 & 1.020 & 1.594 & 0.071 & 2.489 & 0.645 & \\
\hline 665 & V1 & 224 & $12 \mathrm{~B}$ & V1 & 4.239 & 4.657 & 0.207 & 2.158 & 0.129 & 22.498 & 0.442 & \\
\hline 672 & $\mathrm{U} 1$ & 225 & 00 & $\mathrm{U} 1$ & -2.292 & 4.131 & 0.446 & 2.032 & 0.115 & 9.269 & 0.225 & \\
\hline 672 & V1 & 225 & 00 & V1 & 1.862 & 3.175 & 0.484 & 1.782 & 0.088 & 6.553 & 0.831 & \\
\hline 672 & $\mathrm{U} 2$ & 225 & 00 & $\mathrm{U} 2$ & 2.593 & 1.294 & 0.162 & 1.138 & 0.026 & 7.993 & 0.370 & \\
\hline 672 & V2 & 225 & 00 & V2 & 1.827 & 1.548 & 0.319 & 1.244 & 0.032 & 4.853 & 0.171 & \\
\hline 672 & U3 & 225 & 00 & U3 & -1.224 & 2.807 & 0.664 & 1.676 & 0.078 & 4.227 & 0.156 & \\
\hline 672 & V3 & 225 & 00 & V3 & 0.056 & 2.468 & 1.027 & 1.571 & 0.069 & 2.403 & 1.141 & \\
\hline 678 & U1 & 225 & 12 & U1 & -2.908 & 2.107 & 0.200 & 1.452 & 0.043 & 10.520 & 2.017 & \\
\hline 678 & V1 & 225 & 12 & V1 & -0.514 & 4.466 & 0.963 & 2.113 & 0.091 & 4.639 & 0.599 & \\
\hline 679 & U1 & 225 & $12 \mathrm{~B}$ & U1 & -0.304 & 0.984 & 0.938 & 0.992 & 0.027 & 1.049 & 0.020 & \\
\hline 679 & V1 & 225 & $12 \mathrm{~B}$ & V1 & 1.368 & 3.528 & 0.666 & 1.878 & 0.098 & 5.300 & 3.586 & \\
\hline 679 & $\mathrm{U} 2$ & 225 & $12 \mathrm{~B}$ & $\mathrm{U} 2$ & 0.205 & 4.244 & 1.018 & 2.060 & 0.118 & 4.168 & 0.180 & \\
\hline 679 & V2 & 225 & $12 \mathrm{~B}$ & V2 & -0.366 & 2.400 & 0.973 & 1.549 & 0.067 & 2.467 & 0.110 & \\
\hline 686 & U1 & 226 & 00 & U1 & -1.848 & 2.054 & 0.379 & 1.433 & 0.042 & 5.426 & 0.400 & \\
\hline & V1 & & & V1 & 0.512 & 9.929 & 0.994 & 3.151 & 0.203 & 9.988 & 1.110 & \\
\hline & $\mathrm{U} 2$ & & & $\mathrm{U} 2$ & -1.547 & 0.632 & 0.210 & 0.795 & 0.013 & 3.012 & 0.042 & \\
\hline & V2 & & & V2 & 0.793 & 1.671 & 0.737 & 1.293 & 0.034 & 2.267 & 0.536 & 4 \\
\hline 692 & U1 & 226 & 12 & U1 & -2.040 & 5.718 & 0.588 & 2.391 & 0.159 & 9.722 & 0.696 & \\
\hline & V1 & & & V1 & -3.944 & 1.533 & 0.090 & 1.238 & 0.043 & 17.044 & 0.851 & \\
\hline 693 & $\mathrm{U} 1$ & 226 & $12 \mathrm{~B}$ & U1 & -0.193 & 2.576 & 1.006 & 1.605 & 0.053 & 2.561 & 0.039 & 4 \\
\hline & V1 & & & V1 & 0.784 & 6.492 & 0.931 & 2.548 & 0.132 & 6.974 & 0.834 & \\
\hline & $\mathrm{U} 2$ & & & $\mathrm{U} 2$ & -1.118 & 2.566 & 0.685 & 1.602 & 0.071 & 3.745 & 0.897 & 3 \\
\hline & V2 & & & V2 & 0.299 & 3.669 & 1.003 & 1.916 & 0.102 & 3.657 & 0.419 & \\
\hline
\end{tabular}




\begin{tabular}{|c|c|c|c|c|c|c|c|c|c|c|c|c|}
\hline & U3 & & & U3 & 1.630 & 1.771 & 0.405 & 1.331 & 0.049 & 4.378 & 0.082 & 36 \\
\hline & v3 & & & v3 & 2.155 & 3.408 & 0.428 & 1.846 & 0.095 & 7.956 & 0.268 & 36 \\
\hline 700 & U1 & 227 & 00 & U1 & 0.032 & 3.759 & 1.010 & 1.939 & 0.038 & 3.722 & 0.184 & 100 \\
\hline & v1 & & & v1 & -1.080 & 4.942 & 0.816 & 2.223 & 0.049 & 6.059 & 0.219 & 100 \\
\hline 701 & U1 & 227 & $O O B$ & U1 & 2.173 & 1.052 & 0.183 & 1.026 & 0.029 & 5.746 & 0.197 & -36 \\
\hline & v1 & & & v1 & -1.351 & 1.338 & 0.428 & 1.157 & 0.037 & 3.127 & 0.537 & 36 \\
\hline & $\mathrm{U} 2$ & & & U2 & 1.158 & 1.506 & 0.537 & 1.227 & 0.042 & 2.805 & 0.267 & 36 \\
\hline & v2 & & & v2 & 1.873 & 2.564 & 0.427 & 1.601 & 0.071 & 6.001 & 0.523 & 36 \\
\hline & U3 & & & U3 & -1.600 & 1.540 & 0.379 & 1.241 & 0.043 & 4.058 & 0.080 & 36 \\
\hline & v3 & & & v3 & 1.126 & 1.630 & 0.571 & 1.277 & 0.045 & 2.853 & 1.213 & 36 \\
\hline 708 & U1 & 227 & 12 & $\mathrm{U} 1$ & -0.096 & 0.619 & 1.013 & 0.787 & 0.017 & 0.611 & 0.020 & 36 \\
\hline & v1 & & & v1 & -0.064 & 2.281 & 1.027 & 1.510 & 0.063 & 2.221 & 0.487 & 36 \\
\hline & U2 & & & U2 & 4.004 & 2.054 & 0.114 & 1.433 & 0.057 & 18.027 & 0.782 & 36 \\
\hline & v2 & & & v2 & -0.447 & 2.141 & 0.939 & 1.463 & 0.059 & 2.281 & 1.058 & 36 \\
\hline 715 & U1 & 228 & 00 & U1 & 0.002 & 7.798 & 1.021 & 2.792 & 0.159 & 7.639 & 0.047 & 49 \\
\hline & $\mathrm{V} 1$ & & & V1 & 0.769 & 16.747 & 0.985 & 4.092 & 0.342 & 16.998 & 0.406 & 49 \\
\hline & $\mathrm{U} 2$ & & & U2 & -0.089 & 3.699 & 1.019 & 1.923 & 0.075 & 3.632 & 0.055 & 49 \\
\hline & v2 & & & $\mathrm{v} 2$ & -1.752 & 2.052 & 0.404 & 1.432 & 0.042 & 5.080 & 1.126 & 49 \\
\hline & U3 & & & U3 & 0.238 & 3.210 & 1.010 & 1.792 & 0.089 & 3.178 & 0.202 & 36 \\
\hline & v3 & & & v3 & -1.078 & 2.301 & 0.677 & 1.517 & 0.064 & 3.399 & 6.256 & 36 \\
\hline & U4 & & & U4 & 0.219 & 13.226 & 1.017 & 3.637 & 0.270 & 13.005 & 0.335 & 49 \\
\hline & V4 & & & V4 & -0.666 & 7.678 & 0.964 & 2.771 & 0.157 & 7.964 & 0.646 & 49 \\
\hline 716 & U1 & 228 & $00 \mathrm{~B}$ & U1 & 2.141 & 3.264 & 0.421 & 1.807 & 0.091 & 7.759 & 0.607 & 36 \\
\hline & v1 & & & v1 & 1.505 & 2.624 & 0.545 & 1.620 & 0.073 & 4.816 & 0.555 & 36 \\
\hline 721 & U1 & 228 & 12 & U1 & 2.536 & 4.723 & 0.426 & 2.173 & 0.074 & 11.083 & 0.205 & 64 \\
\hline & v1 & & & V1 & -1.235 & 5.815 & 0.802 & 2.411 & 0.091 & 7.251 & 0.947 & 64 \\
\hline 722 & U1 & 228 & $12 \mathrm{~B}$ & U1 & -0.825 & 2.197 & 0.780 & 1.482 & 0.061 & 2.817 & 0.063 & 36 \\
\hline & v1 & & & v1 & 0.406 & 3.183 & 0.977 & 1.784 & 0.088 & 3.259 & 0.735 & 36 \\
\hline & U2 & . & & U2 & 2.966 & 8.954 & 0.510 & 2.992 & 0.183 & 17.566 & 0.609 & 49 \\
\hline & v2 & & & v2 & -4.208 & 5.180 & 0.227 & 2.276 & 0.106 & 22.781 & 2.135 & 49 \\
\hline 729 & U1 & 22.9 & 00 & U1 & 0.847 & 3.181 & 0.830 & 1.784 & 0.065 & 3.833 & 0.324 & 49 \\
\hline & V1 & & & V1 & -2.231 & 2.666 & 0.351 & 1.633 & 0.054 & 7.587 & 1.997 & 49 \\
\hline & U2 & & & U2 & -2.687 & 1.801 & 0.201 & 1.342 & 0.050 & 8.969 & 0.209 & 36 \\
\hline & v2 & & & V2 & 0.876 & 5.979 & 0.909 & 2.445 & 0.166 & 6.581 & 1.689 & 36 \\
\hline 735 & v1 & 229 & 12 & $\mathrm{U} 1$ & 0.208 & 2.128 & 1.007 & 1.459 & 0.059 & 2.112 & 0.019 & 36 \\
\hline & v1 & & & V1 & 1.218 & 3.839 & 0.736 & 1.959 & 0.107 & 5.215 & 1.100 & 36 \\
\hline 736 & U1 & 229 & $12 \mathrm{~B}$ & Ul & -1.356 & 0.866 & 0.322 & 0.931 & 0.018 & 2.688 & 0.131 & 49 \\
\hline & V1 & & & V1 & -0.648 & 2.002 & 0.841 & 1.415 & 0.041 & 2.381 & 0.776 & 49 \\
\hline & $\mathrm{U} 2$ & & & U2 & -0.414 & 0.866 & 0.849 & 0.931 & 0.018 & 1.020 & 0.205 & 49 \\
\hline & v2 & & & $\mathrm{V} 2$ & -3.609 & 1.838 & 0.124 & 1.356 & 0.038 & 14.825 & 1.245 & 49 \\
\hline
\end{tabular}




\begin{tabular}{|c|c|c|c|c|c|c|c|c|c|c|c|c|c|}
\hline & & U3 & & & U3 & -1.171 & 3.419 & 0.724 & 1.849 & 0.070 & 4.720 & 0.933 & 49 \\
\hline & & V3 & & & V3 & 0.526 & 2.098 & 0.900 & 1. 448 & 0.043 & 2.331 & 0.096 & 49 \\
\hline & 744 & U1 & 230 & 00 & U1 & -2.46 & 21.45 & 0.78 & 4.63 & 0.18 & 27.3 & 1.47 & 121 \\
\hline & & $\mathrm{V} 1$ & & & V1 & -2.18 & 7.24 & 0.61 & 2.69 & 0.06 & 11.92 & 1.65 & 121 \\
\hline & & $\mathrm{U} 2$ & & & U2 & 1.64 & 8.33 & 0.76 & 2.89 & 0.07 & 10.95 & 1.42 & 121 \\
\hline & & $\mathrm{V} 2$ & & & v2 & -2.84 & 6.07 & 0.43 & 2.46 & 0.05 & 14.06 & 0.82 & 121 \\
\hline & & บ3 & & & U3 & -1.40 & 5.69 & 0.75 & 2.39 & 0.05 & 7.61 & 0.23 & 121 \\
\hline & & v3 & & & V3 & -0.01 & 5.66 & 1.01 & 2.38 & 0.05 & 5.62 & 1.04 & 121 \\
\hline & 750 & U1 & 230 & 12 & U1 & 0.81 & 5.17 & 0.90 & 2.27 & 0.04 & 5.78 & 0.21 & 121 \\
\hline & & V1 & & & V1 & -0.64 & 1.80 & 0.82 & 1.34 & 0.01 & 2.19 & 0.83 & 121 \\
\hline & & $\mathrm{U} 2$ & & & $\mathrm{U} 2$ & -2.23 & 3.73 & 0.43 & 1.93 & 0.08 & 8.60 & 0.92 & 49 \\
\hline & & V2 & & & $\mathrm{V} 2$ & -2.38 & 2.03 & 0.27 & 1.42 & 0.04 & 7.63 & 2.09 & 49 \\
\hline & & U3 & & & U3 & -1.27 & 4.57 & 0.75 & 2.14 & 0.07 & 6.12 & 0.47 & 64 \\
\hline & & v3 & & & V3 & -2.79 & 3.24 & 0.29 & 1.80 & 0.05 & 10.98 & 0.86 & 64 \\
\hline & 751 & U1 & 230 & $12 \mathrm{~B}$ & U1 & 1.23 & 5.10 & 0.78 & 2.26 & 0.10 & 6.50 & 0.29 & 49 \\
\hline & & V1 & & & v1 & 0.61 & 1.42 & 0.80 & 1.19 & 0.03 & 1.76 & 0.08 & 49 \\
\hline & 758 & $\mathrm{U1}$ & 231 & 00 & $\mathrm{U1}$ & 0.32 & 13.21 & 1.01 & 3.64 & 0.27 & 13.05 & 0.91 & 49 \\
\hline & & V1 & & & V1 & -1.59 & 2.41 & 0.49 & 1.55 & 0.05 & 4.91 & 0.28 & 49 \\
\hline 心 & 772 & U1 & 232 & 00 & U1 & -0.251 & 3.701 & 1.003 & 1.924 & 0.076 & 3.689 & 0.185 & 49 \\
\hline & & V1 & & & v1 & -0.275 & 3.358 & 0.998 & 1.833 & 0.069 & 3.366 & 0.234 & 49 \\
\hline & 773 & U1 & 232 & $O O B$ & U1 & -0.957 & 1.246 & 0.585 & 1.116 & 0.035 & 2.128 & 0.381 & 36 \\
\hline & & V1 & & & VI & -0.308 & 1.602 & 0.969 & 1.266 & 0.045 & 1.653 & 0.648 & 36 \\
\hline & 779 & U1 & 232 & 12 & $\mathrm{U} 1$ & -0.997 & 1.568 & 0.620 & 1.252 & 0.032 & 2.530 & 0.083 & 49 \\
\hline & & VI & & & V1 & 0.099 & 0.792 & 1.008 & 0.890 & 0.016 & 0.786 & 0.628 & 49 \\
\hline & & $\mathrm{U} 2$ & & & U2 & -0.703 & 1.891 & 0.806 & 1.375 & 0.039 & 2.348 & 0.126 & 49 \\
\hline & & v2 & & & V2 & 0.620 & 1.675 & 0.827 & 1.294 & 0.034 & 2.025 & 0.149 & 49 \\
\hline & & U3 & & & U3 & 1.008 & 4.044 & 0.812 & 2.011 & 0.083 & 4.978 & 0.087 & 49 \\
\hline & & V3 & & & V3 & -1.665 & 15.415 & 0.863 & 3.926 & 0.315 & 17.872 & 0.507 & 49 \\
\hline & 780 & $\mathrm{U1}$ & 232 & $12 \mathrm{~B}$ & U1 & -2.328 & 1.048 & 0.163 & 1.023 & 0.029 & 6.439 & 0.203 & 36 \\
\hline & & V1 & & & v1 & -0.860 & 3.560 & 0.847 & 1.887 & 0.099 & 4.202 & 0.385 & 36 \\
\hline & 787 & U1 & 233 & 00 & U1 & 0.372 & 10.538 & 1.003 & 3.246 & 0.165 & 10.512 & 0.776 & 64 \\
\hline & & v1 & & & V1 & -3.178 & 34.192 & 0.781 & 5.847 & 0.534 & 43.761 & 0.860 & 64 \\
\hline & & $\mathrm{u} 2$ & & & $\mathrm{U} 2$ & -3.479 & 20.093 & 0.635 & 4.483 & 0.558 & 31.637 & 0.662 & 36 \\
\hline & & $\mathrm{v} 2$ & & & V2 & 3.058 & 3.663 & 0.284 & 1.914 & 0.102 & 12.914 & 0.429 & 36 \\
\hline & & U3 & & & U3 & 0.647 & 4.546 & 0.933 & 2.132 & 0.093 & 4.871 & 0.145 & 49 \\
\hline & & V3 & & & V3 & -1.399 & 5.599 & 0.752 & 2.366 & 0.114 & 7.442 & 0.468 & 49 \\
\hline & & U4 & & & U4 & -1.099 & 0.939 & 0.441 & 0.969 & 0.019 & 2.128 & 0.071 & 49 \\
\hline & & V4 & & & V4 & -0.742 & 2.463 & 0.831 & 1.569 & 0.050 & 2.963 & 0.422 & 49 \\
\hline & 793 & U1 & 233 & 12 & $\mathrm{U} 1$ & 0.290 & 9.255 & 1.011 & 3.042 & 0.189 & 9.151 & 0.208 & 49 \\
\hline & & V1 & & & V1 & -2.905 & 1.198 & 0.125 & 1.095 & 0.024 & 9.612 & 0.340 & 49 \\
\hline
\end{tabular}




\begin{tabular}{|c|c|c|c|c|c|c|c|c|c|c|c|c|}
\hline & U2 & & & U2 & -3.899 & 9.192 & 0.381 & 3.032 & 0.255 & 24.139 & 3.244 & 36 \\
\hline & v2 & & & v2 & -4.938 & 9.537 & 0.283 & 3.088 & 0.265 & 33.658 & 0.701 & 36 \\
\hline 794 & U1 & 233 & $12 \mathrm{~B}$ & U1 & -1.062 & 1.714 & 0.613 & 1.309 & 0.048 & 2.794 & 0.091 & 36 \\
\hline & V1 & & & v1 & 0.910 & 9.409 & 0.943 & 3.067 & 0.261 & 9.975 & 0.755 & 36 \\
\hline & U2 & & & $\mathrm{U} 2$ & 1.276 & 5.146 & 0.772 & 2.268 & 0.105 & 6.669 & 0.140 & 49 \\
\hline & V2 & & & V2 & -1.232 & 7.327 & 0.843 & 2.707 & 0.150 & 8.694 & 3.572 & 49 \\
\hline 801 & U1 & 234 & 00 & U1 & 1.067 & 5.621 & 0.851 & 2.371 & 0.156 & 6.604 & 0.333 & 36 . \\
\hline & V1 & & & v1 & -3.939 & 21.255 & 0.587 & 4.610 & 0.590 & 36.182 & 0.781 & 36 \\
\hline & $\mathrm{U} 2$ & & & $\mathrm{U} 2$ & 0.122 & 6.405 & 1.026 & 2.531 & 0.178 & 6.242 & 0.141 & 36 \\
\hline & v2 & & & v2 & -1.457 & 3.058 & 0.600 & 1.749 & 0.085 & 5.097 & 0.279 & 36 \\
\hline & U3 & & & U3 & 0.192 & 2.176 & 1.003 & 1.475 & 0.044 & 2.168 & 0.157 & 49 \\
\hline & V3 & & & v3 & -1.172 & 1.564 & 0.538 & 1.250 & 0.032 & 2.906 & 0.331 & 49 \\
\hline & U4 & & & U4 & 0.107 & 1.674 & 1.014 & 1.294 & 0.034 & 1.651 & 0.175 & 49 \\
\hline & V4 & & & V4 & -0.675 & 1.709 & 0.802 & 1.307 & 0.035 & 2.129 & 0.387 & 49 \\
\hline 802 & U1 & 234 & $O O B$ & $\mathrm{U} 1$ & 2.750 & 25.485 & 0.784 & 5.048 & 0.520 & 32.527 & 0.560 & 49 \\
\hline & V1 & & & v1 & -1.517 & 23.067 & 0.927 & 4.803 & 0.471 & 24.897 & 0.958 & 49 \\
\hline & $\mathrm{U} 2$ & & & $\mathrm{u} 2$ & 1.526 & 5.308 & 0.705 & 2.304 & 0.108 & 7.527 & 0.436 & 49 \\
\hline & V2 & & & v2 & 0.167 & 3.355 & 1.012 & 1.832 & 0.068 & 3.315 & 0.561 & 49 \\
\hline 807 & U1 & 234 & 12 & U1 & 0.801 & 3.587 & 0.869 & 1.894 & 0.100 & 4.129 & 0.196 & 36 \\
\hline & V1 & & & v1 & -1.850 & 2.900 & 0.465 & 1.703 & 0.081 & 6.241 & 0.962 & 36 \\
\hline 808 & $\mathrm{U} 1$ & 234 & $12 \mathrm{~B}$ & $\mathrm{U} 1$ & 0.231 & 0.954 & 0.966 & 0.977 & 0.019 & 0.988 & 0.059 & 49 \\
\hline & V1 & & & v1 & 1.082 & 2.613 & 0.700 & 1.617 & 0.053 & 3.731 & 0.360 & 49 \\
\hline & $\mathrm{U} 2$ & & & $\mathrm{U} 2$ & 0.252 & 1.318 & 0.980 & 1.148 & 0.037 & 1.345 & 0.118 & 36 \\
\hline & V2 & & & $\mathrm{v} 2$ & 0.001 & 1.599 & 1.029 & 1.265 & 0.044 & 1.555 & 0.208 & 36 \\
\hline & U3 & & & U3 & 1.097 & 3.271 & 0.746 & 1.809 & 0.091 & 4.383 & 1.334 & 36 \\
\hline & V3 & & & v3 & -5.169 & 2.324 & 0.080 & 1.524 & 0.065 & 28.974 & 0.595 & 36 \\
\hline 815 & U1 & 235 & 00 & $\mathrm{U} 1$ & -1.388 & 2.067 & 0.525 & 1.438 & 0.057 & 3.936 & 0.119 & 36 \\
\hline & V1 & & & V1 & 1.414 & 1.887 & 0.492 & 1.374 & 0.052 & 3.834 & 1.078 & 36 \\
\hline 816 & U1 & 235 & $O O B$ & U1 & 0.451 & 7.244 & 0.992 & 2.692 & 0.148 & 7.300 & 0.199 & 49 \\
\hline & V1 & & & V1 & -3.724 & 16.015 & 0.542 & 4.002 & 0.327 & 29.556 & 1.378 & 49 \\
\hline & $\mathrm{U} 2$ & & & $\mathrm{U} 2$ & -0.582 & 6.522 & 0.965 & 2.554 & 0.102 & 6.760 & 0.350 & 64 \\
\hline & v2 & & & v2 & -1.901 & 8.036 & 0.697 & 2.835 & 0.126 & 11.524 & 0.823 & 64 \\
\hline & U3 & & & U3 & 0.406 & 10.479 & 1.005 & 3.237 & 0.214 & 10.430 & 1.146 & 49 \\
\hline & V3 & & & V3 & 2.208 & 6.515 & 0.579 & 2.553 & 0.133 & 11.258 & 0.638 & 49 \\
\hline 822 & $\mathrm{U} 1$ & 235 & 12 & $\mathrm{U} 1$ & -2.046 & 2.821 & 0.406 & 1.679 & 0.058 & 6.948 & 0.271 & 49 \\
\hline & v1 & & & v1 & 0.358 & 1.172 & 0.918 & 1.083 & 0.024 & 1.277 & 0.983 & 49 \\
\hline & U2 & & & U2 & -0.530 & 2.207 & 0.904 & 1.486 & 0.045 & 2.442 & 0.309 & 49 \\
\hline & V2 & & & v2 & -1.217 & 2.417 & 0.628 & 1.555 & 0.049 & 3.848 & 1.195 & 49 \\
\hline & U3 & & & ט3 & 2.118 & 4.175 & 0.486 & 2.043 & 0.065 & 8.595 & 0.148 & 64 \\
\hline & V3 & & & v3 & 1.102 & 4.408 & 0.794 & 2.099 & 0.069 & 5.553 & 0.178 & 64 \\
\hline
\end{tabular}




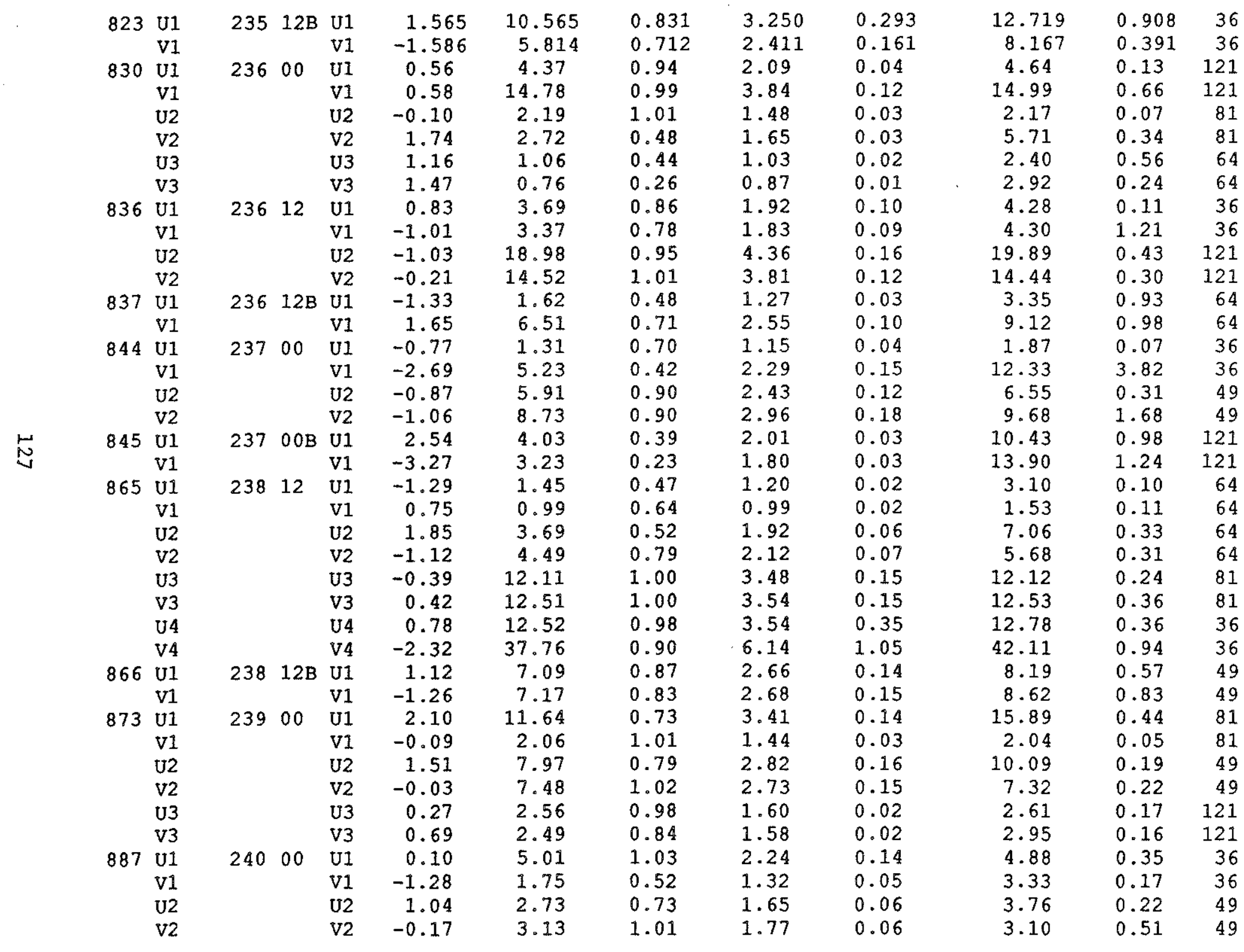




\begin{tabular}{|c|c|c|c|c|c|c|c|c|c|c|c|c|}
\hline 888 & $8 \mathrm{U1}$ & 240 & OOB & U1 & -1.51 & 1.24 & 0.35 & 1.11 & 0.03 & 3.49 & 0.22 & 36 \\
\hline & v1 & & & V1 & 1.06 & 0.92 & 0.46 & 0.96 & 0.03 & 2.02 & 3.45 & 36 \\
\hline 901 & $1 \mathrm{U} 1$ & 241 & 00 & U1 & -0.96 & 1.17 & 0.56 & 1.08 & 0.01 & 2.09 & 0.10 & 121 \\
\hline & V1 & & & V1 & 2.58 & 5.47 & 0.45 & 2.34 & 0.05 & 12.11 & 3.51 & 121 \\
\hline 902 & $2 \mathrm{U1}$ & 241 & OOB & U1 & -0.81 & 2.20 & 0.78 & 1.48 & 0.04 & 2.81 & 0.58 & 49 \\
\hline & v1 & & & V1 & -0.42 & 1.12 & 0.88 & 1.06 & 0.02 & 1.27 & 0.02 & 49 \\
\hline & $\mathrm{U} 2$ & & & U2 & -0.64 & 6.18 & 0.95 & 2.49 & 0.05 & 6.53 & 0.27 & 121 \\
\hline & v2 & & & V2 & -0.63 & 2.77 & 0.88 & 1.66 & 0.02 & 3.14 & 1.41 & 121 \\
\hline 916 & $6 \mathrm{U} 1$ & 242 & 00 & U1 & 5.681 & 15.348 & 0.324 & 3.918 & 0.240 & 47.383 & 2.809 & 64 \\
\hline & $\mathrm{V} 1$ & & & V1 & -4.854 & 7.233 & 0.236 & 2.690 & 0.113 & 30.686 & 0.390 & 64 \\
\hline & $\mathrm{U} 2$ & & & $\mathrm{U} 2$ & -3.756 & 4.471 & 0.242 & 2.114 & 0.091 & 18.490 & 0.378 & 49 \\
\hline & V2 & & & v2 & -0.882 & 1.269 & 0.628 & 1.126 & 0.026 & 2.020 & 0.313 & 49 \\
\hline & U3 & & & U3 & -3.196 & 4.716 & 0.318 & 2.172 & 0.096 & 14.833 & 0.803 & 49 \\
\hline & v3 & & & v3 & -0.161 & 3.019 & 1.012 & 1.738 & 0.062 & 2.983 & 0.557 & 49 \\
\hline 930 & U1 & 243 & 00 & U1 & -0.490 & 3.466 & 0.953 & 1.862 & 0.071 & 3.635 & 0.537 & 49 \\
\hline & V1 & & & v1 & -5.141 & 5.162 & 0.164 & 2.272 & 0.105 & 31.484 & 1.683 & 49 \\
\hline & $\mathrm{U} 2$ & & & U2 & -1.576 & 0.371 & 0.130 & 0.609 & 0.008 & 2.849 & 0.093 & 49 \\
\hline & v2 & & & v2 & -1.124 & 2.734 & 0.694 & 1.654 & 0.056 & 3.942 & 1.078 & 49 \\
\hline 951 & U1 & 244 & 12 & U1 & 1.237 & 1.101 & 0.423 & 1.049 & 0.031 & 2.601 & 0.085 & 36 \\
\hline & V1 & & & V1 & -1.267 & 6.150 & 0.811 & 2.480 & 0.171 & 7.585 & 0.852 & 36 \\
\hline & U2 & & & U2 & 0.349 & 2.928 & 0.986 & 1.711 & 0.081 & 2.968 & 1.318 & 36 \\
\hline & v2 & & & v2 & -3.516 & 1.980 & 0.139 & 1.407 & 0.055 & 14.288 & 1.592 & 36 \\
\hline & U3 & & & U3 & 0.619 & 1.225 & 0.778 & 1.107 & 0.034 & 1.574 & 0.305 & 36 \\
\hline & v3 & & & v3 & -4.778 & 2.028 & 0.082 & 1.424 & 0.056 & 24.798 & 3.106 & 36 \\
\hline 952 & U1 & 244 & $12 \mathrm{~B}$ & U1 & -1.622 & 26.680 & 0.927 & 5.165 & 0.544 & 28.766 & 3.312 & 49 \\
\hline & v1 & & & v1 & 3.191 & 17.830 & 0.645 & 4.223 & 0.364 & 27.646 & 0.697 & 49 \\
\hline & U2 & & & U2 & 4.617 & 138.356 & 0.882 & 11.762 & 2.824 & 156.847 & 10.129 & 49 \\
\hline & v2 & & & v2 & 1.996 & 78.808 & 0.971 & 8.877 & 1.608 & 81.183 & 1.593 & 49 \\
\hline 959 & U1 & 245 & 00 & $\mathrm{U} 1$ & -3.716 & 2.304 & 0.143 & 1.518 & 0.047 & 16.067 & 2.878 & 49 \\
\hline & V1 & & & v1 & -0.855 & 0.900 & 0.558 & 0.949 & 0.018 & 1.613 & 0.168 & 49 \\
\hline & $\mathrm{U} 2$ & & & $\mathrm{U} 2$ & -2.946 & 1.091 & 0.112 & 1.044 & 0.030 & 9.738 & 1.237 & 36 \\
\hline & v2 & & & v2 & -0.605 & 0.398 & 0.529 & 0.631 & 0.011 & 0.753 & 2.194 & 36 \\
\hline & U3 & & & U3 & 0.891 & 3.809 & 0.842 & 1.952 & 0.078 & 4.526 & 0.146 & 49 \\
\hline & v3 & & & v3 & -4.573 & 9.615 & 0.317 & 3.101 & 0.196 & 30.330 & 1.191 & 49 \\
\hline 973 & $\mathrm{U} 1$ & 246 & 00 & U1 & -1.825 & 0.692 & 0.173 & 0.832 & 0.019 & 4.002 & 0.293 & 36 \\
\hline & V1 & & & v1 & -3.465 & 2.971 & 0.199 & 1.724 & 0.083 & 14.894 & 1.895 & 36 \\
\hline & U2 & & & $\mathrm{U} 2$ & -1.298 & 0.870 & 0.344 & 0.933 & 0.024 & 2.531 & 0.945 & 36 \\
\hline & v2 & & & v2 & -3.432 & 1.706 & 0.127 & 1.306 & 0.047 & 13.435 & 1.275 & 36 \\
\hline & U3 & & & U3 & -1.712 & 2.025 & 0.412 & 1.423 & 0.041 & 4.916 & 0.092 & 49 \\
\hline & v3 & & & v3 & -0.704 & 2.304 & 0.837 & 1.518 & 0.047 & 2.753 & 1.024 & 49 \\
\hline
\end{tabular}


ஓ

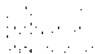




\begin{tabular}{|c|c|c|c|c|c|c|c|c|c|c|c|c|}
\hline & U2 & & & U2 & -2.63 & 10.26 & 0.60 & 3.20 & 0.13 & 17.07 & 0.25 & 81 \\
\hline & v2 & & & v2 & 0.37 & 18.18 & 1.00 & 4.26 & 0.22 & 18.10 & 0.83 & 81 \\
\hline 1017 & U1 & 249 & $O O B$ & U1 & 3.62 & 3.57 & 0.21 & 1.89 & 0.10 & 16.60 & 2.42 & 36 \\
\hline & V1 & & & V1 & 1.65 & 1.61 & 0.38 & 1.27 & 0.04 & 4.30 & 0.19 & 36 \\
\hline 1030 & U1 & 250 & 00 & U1 & -0.17 & 1.67 & 0.99 & 1.29 & 0.01 & 1.68 & 0.05 & 121 \\
\hline & v1 & & & V1 & -1.58 & 3.09 & 0.56 & 1.76 & 0.03 & 5.55 & 0.47 & 121 \\
\hline 1031 & U1 & 250 & $\mathrm{OOB}$ & U1 & 0.73 & 3.23 & 0.87 & 1.80 & 0.05 & 3.72 & 0.10 & 64 \\
\hline & V1 & & & v1 & -0.26 & 2.98 & 0.99 & 1.73 & 0.05 & 3.01 & 0.18 & 64 \\
\hline & $\mathrm{U} 2$ & & & U2 & 3.99 & 20.70 & 0.57 & 4.55 & 0.58 & 36.01 & 1.54 & 36 \\
\hline & V2 & & & v2 & 0.12 & 4.12 & 1.02 & 2.03 & 0.11 & 4.02 & 0.20 & 36 \\
\hline & U3 & & & U3 & -1.28 & 3.48 & 0.69 & 1.87 & 0.10 & 5.04 & 0.16 & 36 \\
\hline & v3 & & & v3 & -3.00 & 1.37 & 0.13 & 1.17 & 0.04 & 10.36 & 0.37 & 36 \\
\hline 1051 & U1 & 251 & 12 & U1 & -1.69 & 26.00 & 0.91 & 5.10 & 0.21 & 28.63 & 0.48 & 121 \\
\hline & V1 & & & v1 & 0.95 & 45.08 & 0.99 & 6.71 & 0.37 & 45.60 & 0.42 & 121 \\
\hline 1052 & U1 & 251 & $12 \mathrm{~B}$ & U1 & 5.15 & 29.63 & 0.53 & 5.44 & 0.30 & 55.84 & 2.92 & 100 \\
\hline & v1 & & & V1 & 3.36 & 19.67 & 0.64 & 4.44 & 0.20 & 30.73 & 1.47 & 100 \\
\hline & U2 & & & U2 & 3.38 & 23.39 & 0.68 & 4.84 & 0.48 & 34.33 & 0.63 & 49 \\
\hline & v2 & & & v2 & -3.66 & 26.94 & 0.68 & 5.19 & 0.55 & 39.79 & 0.66 & 49 \\
\hline 1059 & U1 & 252 & 00 & U1 & -1.80 & 1.20 & 0.27 & 1.10 & 0.02 & 4.41 & 0.26 & 49 \\
\hline & V1 & & & v1 & 0.78 & 2.75 & 0.83 & 1.66 & 0.06 & 3.29 & 0.05 & 49 \\
\hline & U2 & & & U2 & 1.40 & 4.14 & 0.69 & 2.03 & 0.08 & 6.03 & 0.60 & 49 \\
\hline & v2 & & & v2 & 2.40 & 2.09 & 0.27 & 1.45 & 0.04 & 7.82 & 0.21 & 49 \\
\hline 1060 & U1 & 252 & $00 \mathrm{~B}$ & U1 & -1.12 & 0.60 & 0.33 & 0.77 & 0.02 & 1.83 & 0.49 & 36 \\
\hline & V1 & & & v1 & -1.08 & 2.42 & 0.69 & 1.55 & 0.07 & 3.52 & 0.98 & 36 \\
\hline 1074 & U1 & 253 & 00 & U1 & -2.50 & 12.47 & 0.68 & 3.53 & 0.25 & 18.46 & 0.10 & 49 \\
\hline & V1 & & & v1 & 3.86 & 9.82 & 0.40 & 3.13 & 0.20 & 24.56 & 0.34 & 49 \\
\hline & U2 & & & $\mathrm{U} 2$ & 0.45 & 67.35 & 1.02 & 8.21 & 1.37 & 66.18 & 0.55 & 49 \\
\hline & v2 & & & v2 & 1.60 & 46.67 & 0.97 & 6.83 & 0.95 & 48.29 & 0.35 & 49 \\
\hline & U3 & & & U3 & 0.52 & 9.49 & 0.99 & 3.08 & 0.19 & 9.56 & 0.18 & 49 \\
\hline & v3 & & & v3 & 1.02 & 17.27 & 0.96 & 4.16 & 0.35 & 17.96 & 1.01 & 49 \\
\hline & U4 & & & U4 & -0.05 & 1.06 & 1.03 & 1.03 & 0.03 & 1.03 & 0.10 & 36 \\
\hline & V4 & & & V4 & -0.47 & 5.77 & 0.99 & 2.40 & 0.16 & 5.83 & 1.46 & 36 \\
\hline
\end{tabular}


Table 17: Atlas-FNOC difference field component statistics - 1-day averages

\begin{tabular}{|c|c|c|c|c|c|}
\hline DAY & $\frac{\text { MEAN }}{(\mathrm{m} / \mathrm{s})}$ & $\frac{\text { VAR }}{\left(\mathrm{m}^{2} / \mathrm{s}^{2}\right)}$ & WVAR & $\frac{\mathrm{SDEV}}{(\mathrm{m} / \mathrm{s})}$ & $\underline{N}$ \\
\hline $224 \mathrm{U}$ & 0.126 & 5.692 & 1.000 & 2.386 & 378 \\
\hline V & 1.355 & 6.333 & 0.777 & 2.516 & 378 \\
\hline $225 \mathrm{U}$ & -0.917 & 5.039 & 0.859 & 2.245 & 340 \\
\hline $\mathrm{V}$ & 0.686 & 4.447 & 0.907 & 2.109 & 340 \\
\hline $226 \mathrm{U}$ & -0.357 & 4.217 & 0.973 & 2.054 & 463 \\
\hline V & 0.000 & 6.518 & 1.002 & 2.553 & 463 \\
\hline $227 \mathrm{U}$ & 0.598 & 5.827 & 0.944 & 2.414 & 499 \\
\hline V & -0.266 & 5.887 & 0.990 & 2.426 & 499 \\
\hline $228 \mathrm{U}$ & 0.695 & 7.846 & 0.944 & 2.801 & 453 \\
\hline V & -0.913 & 8.177 & 0.909 & 2.860 & 453 \\
\hline $229 \mathrm{U}$ & -0.730 & 9.868 & 0.950 & 3.141 & 631 \\
\hline V & -1.307 & 7.141 & 0.808 & 2.672 & 631 \\
\hline $230 \mathrm{U}$ & -0.412 & 11.391 & 0.987 & 3.375 & 695 \\
\hline V & -1.480 & 5.720 & 0.724 & 2.392 & 695 \\
\hline $231 \mathrm{U}$ & -0.233 & 6.686 & 0.999 & 2.586 & 134 \\
\hline $\mathrm{V}$ & -0.766 & 2.903 & 0.837 & 1.704 & 134 \\
\hline $232 \mathrm{U}$ & -0.618 & 6.341 & 0.945 & 2.518 & 466 \\
\hline V & -0.644 & 10.690 & 0.965 & 3.270 & 466 \\
\hline $233 \mathrm{U}$ & 0.065 & 10.250 & 1.001 & 3.201 & 636 \\
\hline V & -1.410 & 12.745 & 0.866 & 3.570 & 636 \\
\hline $234 \mathrm{U}$ & 0.498 & 6.919 & 0.967 & 2.630 & 623 \\
\hline V & -1.116 & 10.804 & 0.898 & 3.287 & 623 \\
\hline $235 \mathrm{U}$ & 0.234 & 5.698 & 0.992 & 2.387 & 662 \\
\hline V & 0.198 & 9.399 & 0.997 & 3.066 & 662 \\
\hline $236 \mathrm{U}$ & 0.275 & 7.607 & 0.992 & 2.758 & 693 \\
\hline V & -0.283 & 10.829 & 0.994 & 3.291 & 693 \\
\hline $237 \mathrm{U}$ & 1.148 & 6.725 & 0.840 & 2.593 & 206 \\
\hline V & -2.644 & 5.662 & 0.449 & 2.380 & 206 \\
\hline $238 \mathrm{U}$ & 0.666 & 7.995 & 0.949 & 2.827 & 545 \\
\hline V & -0.111 & 7.885 & 1.000 & 2.808 & 545 \\
\hline $239 \mathrm{U}$ & 0.743 & 6.408 & 0.923 & 2.531 & 372 \\
\hline V & 0.158 & 3.277 & 0.995 & 1.810 & 372 \\
\hline $240 \mathrm{U}$ & -0.564 & 3.733 & 0.924 & 1.932 & 412 \\
\hline V & 0.486 & 5.241 & 0.959 & 2.289 & 412 \\
\hline $241 \mathrm{U}$ & -0.463 & 12.716 & 0.986 & 3.566 & 453 \\
\hline V & -0.321 & 9.003 & 0.991 & 3.000 & 453 \\
\hline $242 \mathrm{U}$ & -0.301 & 19.147 & 0.999 & 4.376 & 260 \\
\hline V & -2.572 & 8.591 & 0.566 & 2.931 & 260 \\
\hline
\end{tabular}




$\begin{array}{rlllll}243 \mathrm{U} & -1.033 & 2.197 & 0.678 & 1.482 & 98 \\ \mathrm{~V} & -3.132 & 7.982 & 0.451 & 2.825 & 98 \\ 244 \mathrm{U} & -0.054 & 31.594 & 1.003 & 5.621 & 340 \\ \mathrm{~V} & -1.111 & 24.144 & 0.954 & 4.914 & 340 \\ 245 \mathrm{U} & -1.728 & 4.227 & 0.587 & 2.056 & 255 \\ \mathrm{~V} & -2.237 & 5.790 & 0.537 & 2.406 & 255 \\ 246 \mathrm{U} & -1.044 & 14.779 & 0.934 & 3.844 & 378 \\ \mathrm{~V} & -0.441 & 15.709 & 0.990 & 3.963 & 37.8 \\ 247 \mathrm{U} & -1.421 & 10.770 & 0.843 & 3.282 & 612 \\ \mathrm{~V} & -0.263 & 11.738 & 0.996 & 3.426 & 612 \\ 248 \mathrm{U} & -0.839 & 15.860 & 0.958 & 3.982 & 920 \\ \mathrm{~V} & 0.230 & 16.622 & 0.998 & 4.077 & 920 \\ 249 \mathrm{U} & -0.036 & 9.672 & 1.002 & 3.110 & 423 \\ \mathrm{~V} & -0.505 & 7.143 & 0.968 & 2.673 & 423 \\ 250 \mathrm{U} & 0.484 & 7.264 & 0.972 & 2.695 & 257 \\ \cdot \mathrm{V} & -1.211 & 3.925 & 0.730 & 1.981 & 257 \\ 251 \mathrm{U} & 1.031 & 26.992 & 0.964 & 5.195 & 404 \\ \mathrm{~V} & 0.959 & 26.897 & 0.969 & 5.186 & 404 \\ 252 \mathrm{U} & -0.429 & 16.367 & 0.992 & 4.046 & 317 \\ \mathrm{~V} & 1.318 & 15.125 & 0.899 & 3.889 & 317\end{array}$


Table 18: Atlas-FNOC difference field component statistics - 1-day $/ 10^{\circ}$ latitude averages

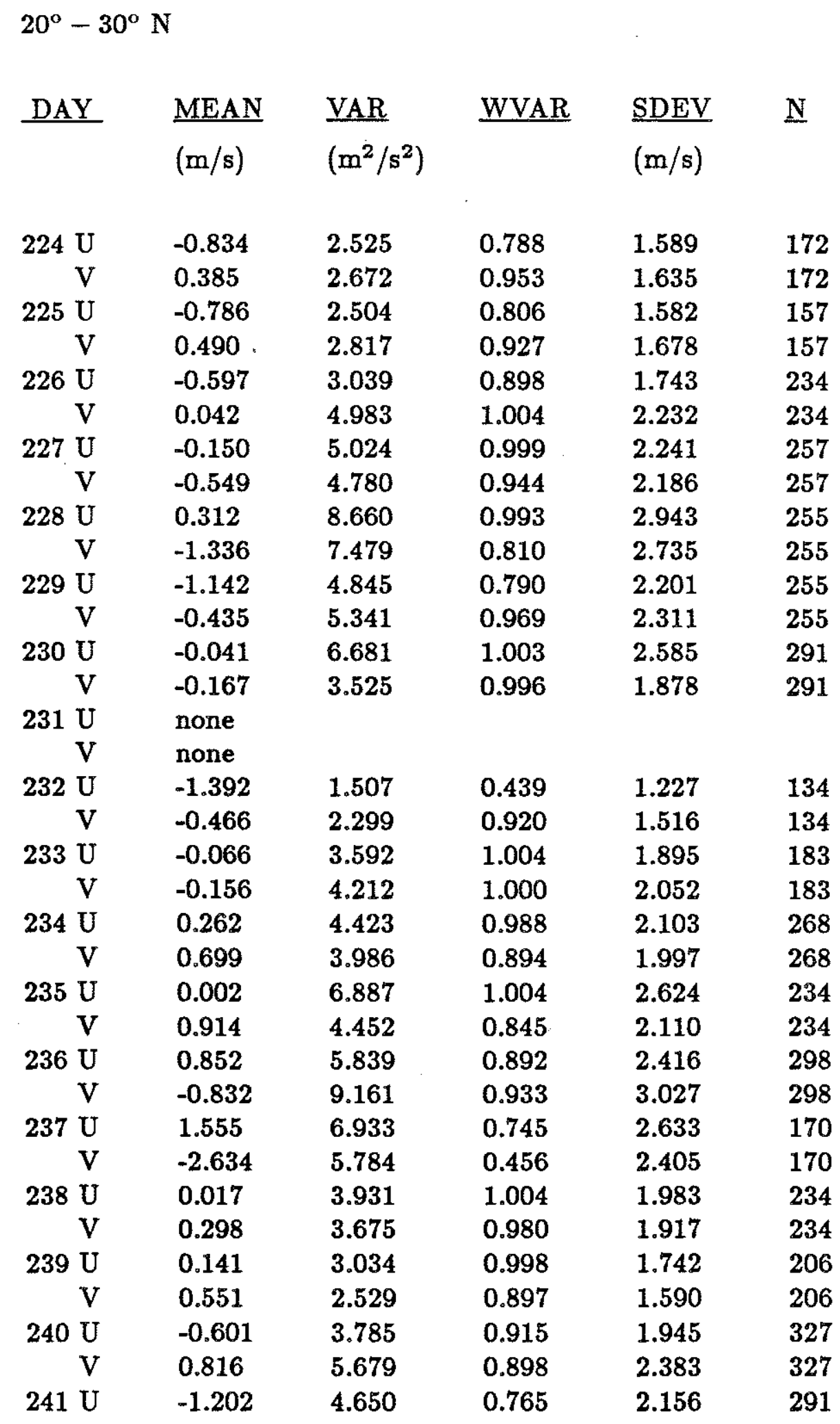




\begin{tabular}{|c|c|c|c|c|}
\hline V & 0.787 & 6.242 & 0.913 & 2.498 \\
\hline $242 \mathrm{U}$ & -2.386 & 3.179 & 0.360 & 1.783 \\
\hline V & -0.642 & 3.082 & 0.890 & 1.755 \\
\hline $243 \mathrm{U}$ & -1.576 & 0.371 & 0.130 & 0.609 \\
\hline $\mathrm{V}$ & -1.124 & 2.734 & 0.694 & 1.654 \\
\hline $244 \mathrm{U}$ & 0.065 & 12.965 & 1.007 & 3.601 \\
\hline $\mathrm{V}$ & -0.846 & 22.692 & 0.976 & 4.764 \\
\hline $245 \mathrm{U}$ & -0.649 & 3.739 & 0.905 & 1.934 \\
\hline V & -2.851 & 7.633 & 0.486 & 2.763 \\
\hline $246 \mathrm{U}$ & -1.603 & 3.584 & 0.584 & 1.893 \\
\hline V & -0.528 & 8.471 & 0.971 & 2.910 \\
\hline $247 \mathrm{U}$ & -1.526 & 3.757 & 0.618 & 1.938 \\
\hline V & -0.120 & 6.578 & 1.001 & 2.565 \\
\hline $248 \mathrm{U}$ & -0.635 & 8.079 & 0.955 & 2.842 \\
\hline V & 0.138 & 5.891 & 1.000 & 2.427 \\
\hline $249 \mathrm{U}$ & -0.018 & 11.852 & 1.003 & 3.443 \\
\hline V & -0.662 & 8.654 & 0.955 & 2.942 \\
\hline $250 \mathrm{U}$ & 0.400 & 8.597 & 0.987 & 2.932 \\
\hline V & -1.527 & 3.852 & 0.625 & 1.963 \\
\hline $251 \mathrm{U}$ & 2.938 & 23.631 & 0.735 & 4.861 \\
\hline V & 2.239 & 14.432 & 0.745 & 3.799 \\
\hline $252 \mathrm{U}$ & 0.223 & 3.268 & 0.993 & 1.808 \\
\hline V & 0.511 & 5.737 & 0.964 & 2.395 \\
\hline
\end{tabular}

$30^{\circ}-40^{\circ} \mathrm{N}$

$\underline{\text { DAY }} \frac{\text { MEAN }}{(\mathrm{m} / \mathrm{s})} \quad \frac{\text { VAR }}{\left(\mathrm{m}^{2} / \mathrm{s}^{2}\right)} \quad \underline{\text { WVAR }} \quad \frac{\text { SDEV }}{(\mathrm{m} / \mathrm{s})} \quad \underline{N}$

\begin{tabular}{|c|c|c|c|c|c|}
\hline $224 U$ & 1.609 & 4.891 & 0.656 & 2.212 & 170 \\
\hline V & 2.228 & 8.987 & 0.647 & 2.998 & 170 \\
\hline $225 \mathrm{U}$ & -0.539 & 6.977 & 0.965 & 2.641 & 183 \\
\hline V & 0.416 & 5.559 & 0.975 & 2.358 & 183 \\
\hline $226 \mathrm{U}$ & 0.248 & 4.449 & 0.991 & 2.109 & 193 \\
\hline V & 0.552 & 5.533 & 0.953 & 2.352 & 193 \\
\hline $227 \mathrm{U}$ & 1.745 & 4.304 & 0.587 & 2.075 & 193 \\
\hline V & -0.150 & 4.316 & 1.000 & 2.077 & 193 \\
\hline $228 \mathrm{U}$ & 1.921 & 6.582 & 0.643 & 2.565 & 198 \\
\hline V & -1.601 & 7.590 & 0.750 & 2.755 & 198 \\
\hline $229 \mathrm{U}$ & 0.551 & 7.064 & 0.963 & 2.658 & 219 \\
\hline $\mathrm{V}$ & -2.257 & 6.544 & 0.564 & 2.558 & 219 \\
\hline $230 \mathrm{U}$ & 0.683 & 9.006 & 0.955 & 3.001 & 219 \\
\hline V & -1.962 & 6.050 & 0.613 & 2.460 & 219 \\
\hline $231 \mathrm{U}$ & -0.550 & 2.757 & 0.911 & 1.660 & 85 \\
\hline
\end{tabular}




\begin{tabular}{|c|c|c|c|c|c|}
\hline & -0.289 & 2.587 & 0.980 & 1.608 & 85 \\
\hline 232 & -0.468 & 7.248 & 0.974 & 2.692 & 268 \\
\hline & -0.128 & 7.583 & 1.002 & 2.754 & 268 \\
\hline 233 & 0.482 & 12.026 & 0.984 & 3.468 & 353 \\
\hline & -1.380 & 11.087 & 0.855 & 3.330 & 353 \\
\hline 234 & 0.701 & 9.741 & 0.955 & 3.121 & 270 \\
\hline & -2.347 & 11.678 & 0.681 & 3.417 & 270 \\
\hline 235 & 0.410 & 5.757 & 0.975 & 2.399 & 294 \\
\hline & -0.092 & 6.947 & 1.002 & 2.636 & 294 \\
\hline 236 & 0.638 & 5.152 & 0.929 & 2.270 & 338 \\
\hline & -0.837 & 9.171 & 0.932 & 3.028 & 338 \\
\hline 237 & 1.777 & 5.343 & 0.631 & 2.311 & 157 \\
\hline & -3.138 & 3.721 & 0.275 & 1.929 & 157 \\
\hline 238 & 0.873 & 9.268 & 0.927 & 3.044 & 279 \\
\hline & -0.663 & 12.782 & 0.970 & 3.575 & 279 \\
\hline 239 & 0.915 & 7.131 & 0.904 & 2.670 & 85 \\
\hline & -0.557 & 5.386 & 0.956 & 2.321 & 85 \\
\hline 240 & -0.422 & 3.550 & 0.963 & 1.884 & 85 \\
\hline & -0.783 & 1.549 & 0.723 & 1.245 & 85 \\
\hline 241 & -2.281 & 5.502 & 0.517 & 2.346 & 98 \\
\hline & -0.651 & 1.235 & 0.750 & 1.111 & 98 \\
\hline 242 & -2.123 & 6.622 & 0.599 & 2.573 & 98 \\
\hline & -3.011 & 7.764 & 0.463 & 2.786 & 98 \\
\hline 243 & -0.490 & 3.466 & 0.953 & 1.862 & 49 \\
\hline & -5.141 & 5.162 & 0.164 & 2.272 & 49 \\
\hline 244 & 1.097 & 66.644 & 0.990 & 8.164 & 121 \\
\hline & -0.418 & 37.485 & 1.004 & 6.123 & 121 \\
\hline 245 & -2.385 & 1.197 & 0.174 & 1.094 & 72 \\
\hline & -2.035 & 3.735 & 0.477 & 1.933 & 72 \\
\hline 246 & 1.333 & 56.002 & 0.982 & 7.483 & 72 \\
\hline & -0.072 & 46.855 & 1.014 & 6.845 & 72 \\
\hline 247 & -0.582 & 32.787 & 0.996 & 5.726 & 149 \\
\hline & -1.095 & 30.133 & 0.968 & 5.489 & 149 \\
\hline 248 & -0.209 & 7.590 & 0.997 & 2.755 & 355 \\
\hline & -1.072 & 6.211 & 0.846 & 2.492 & 355 \\
\hline 249 & 0.810 & 6.214 & 0.910 & 2.493 & 149 \\
\hline & 0.343 & 3.039 & 0.969 & 1.743 & 149 \\
\hline 250 & 0.734 & 3.231 & 0.869 & 1.797 & 64 \\
\hline & -0.260 & 2.985 & 0.993 & 1.728 & 64 \\
\hline 251 & 0.790 & 18.939 & 0.978 & 4.352 & 98 \\
\hline & -1.442 & 19.662 & 0.913 & 4.434 & 98 \\
\hline 252 & -0.277 & 26.826 & 1.004 & 5.179 & 147 \\
\hline & 1.135 & 22.043 & 0.951 & 4.695 & 147 \\
\hline
\end{tabular}




\begin{tabular}{|c|c|c|c|c|c|}
\hline DAY & $\frac{\text { MEAN }}{(\mathrm{m} / \mathrm{s})}$ & $\frac{\text { VAR }}{\left(\mathrm{m}^{2} / \mathrm{s}^{2}\right)}$ & WVAR & $\frac{\mathrm{SDEV}}{(\mathrm{m} / \mathrm{s})}$ & $\underline{\mathbf{N}}$ \\
\hline $224 U$ & -2.292 & 4.131 & 0.446 & 2.032 & 36 \\
\hline V & 1.862 & 3.175 & 0.484 & 1.782 & 36 \\
\hline $225 \mathrm{U}$ & none & & & & \\
\hline V & none & & & & \\
\hline $226 \mathrm{U}$ & -2.040 & 5.718 & 0.588 & 2.391 & 36 \\
\hline V & -3.944 & 1.533 & 0.090 & 1.238 & 36 \\
\hline $227 \mathrm{U}$ & 0.002 & 7.798 & 1.021 & 2.792 & 49 \\
\hline V & 0.769 & 16.747 & 0.985 & 4.092 & 49 \\
\hline $228 \mathrm{U}$ & 0.002 & 7.798 & 1.021 & 2.792 & 49 \\
\hline V & 0.769 & 16.747 & 0.985 & 4.092 & 49 \\
\hline 229. U & -1.850 & 18.249 & 0.847 & 4.272 & 157 \\
\hline V & -1.400 & 8.480 & 0.816 & 2.912 & 157 \\
\hline $230 \mathrm{U}$ & -1.555 & 16.196 & 0.873 & 4.024 & 234 \\
\hline V & -2.224 & 5.273 & 0.517 & 2.296 & 234 \\
\hline $231 \mathrm{U}$ & 0.318 & 13.213 & 1.013 & 3.635 & 49 \\
\hline V & -1.594 & 2.414 & 0.492 & 1.554 & 49 \\
\hline $232 \mathrm{U}$ & -1.015 & 17.260 & 0.953 & 4.155 & 100 \\
\hline V & -0.933 & 32.106 & 0.983 & 5.666 & 100 \\
\hline $233 \mathrm{U}$ & -1.380 & 15.074 & 0.892 & 3.883 & 172 \\
\hline V & -1.881 & 23.696 & 0.874 & 4.868 & 172 \\
\hline $234 \mathrm{U}$ & 0.457 & 5.881 & 0.973 & 2.425 & 121 \\
\hline V & -2.492 & 9.209 & 0.600 & 3.035 & 121 \\
\hline $235 \mathrm{U}$ & 0.964 & 5.375 & 0.856 & 2.318 & 234 \\
\hline V & -0.181 & 15.493 & 1.002 & 3.936 & 234 \\
\hline $236 \mathrm{U}$ & -0.235 & 12.262 & 1.000 & 3.502 & 242 \\
\hline V & 0.184 & 14.743 & 1.002 & 3.840 & 242 \\
\hline $237 \mathrm{U}$ & none & & & & \\
\hline V & none & & & & \\
\hline $238 \mathrm{U}$ & 0.851 & 13.359 & 0.954 & 3.655 & 162 \\
\hline V & 0.163 & 7.306 & 1.003 & 2.703 & 162 \\
\hline $239 \mathrm{U}$ & 2.096 & 11.638 & 0.733 & 3.411 & 81 \\
\hline V & -0.092 & 2.060 & 1.008 & 1.435 & 81 \\
\hline $240 \mathrm{U}$ & none & & & & \\
\hline V & none & & & & \\
\hline $241 \mathrm{U}$ & 1.589 & 32.619 & 0.936 & 5.711 & 113 \\
\hline V & -3.132 & 8.524 & 0.467 & 2.920 & 113 \\
\hline $242 \mathrm{U}$ & 1.589 & 32.619 & 0.936 & 5.711 & 113 \\
\hline V & -3.132 & 8.524 & 0.467 & 2.920 & 113 \\
\hline $243 \mathrm{U}$ & none & & & & \\
\hline
\end{tabular}




$\begin{array}{rlllll}\mathrm{V} & \text { none } & & & & \\ 244 \mathrm{U} & -1.880 & 6.471 & 0.652 & 2.544 & 85 \\ \mathrm{~V} & -2.517 & 5.160 & 0.451 & 2.272 & 85 \\ 245 \mathrm{U} & -3.716 & 2.304 & 0.143 & 1.518 & 49 \\ \mathrm{~V} & -0.855 & 0.900 & 0.558 & 0.949 & 49 \\ 246 \mathrm{U} & \text { none } & & & & \\ \mathrm{V} & \text { none } & & & & \\ 247 \mathrm{U} & -2.156 & 2.277 & 0.330 & 1.509 & 121 \\ \mathrm{~V} & 0.356 & 2.613 & 0.961 & 1.616 & 121 \\ 248 \mathrm{U} & -1.404 & 39.855 & 0.957 & 6.313 & 242 \\ \mathrm{~V} & 2.487 & 38.426 & 0.864 & 6.199 & 242 \\ 249 \mathrm{U} & \text { none } & & & & \\ \mathrm{V} & \text { none } & & & & \\ 250 \mathrm{U} & \text { none } & & & & \\ \mathrm{V} & \text { none } & & & & \\ 251 \mathrm{U} & -1.689 & 25.996 & 0.908 & 5.099 & 121 \\ \mathrm{~V} & 0.946 & 45.078 & 0.989 & 6.714 & 121 \\ 252 \mathrm{U} & -2.499 & 12.473 & 0.676 & 3.532 & 49 \\ \mathrm{~V} & 3.865 & 9.818 & 0.400 & 3.133 & 49\end{array}$


Table 19: Atlas-ENOC difference field vector statistics - area averages

\begin{tabular}{|c|c|c|c|c|c|c|c|c|c|}
\hline \multicolumn{2}{|l|}{ REV } & \multicolumn{2}{|c|}{ TIME } & \multirow{2}{*}{$\begin{array}{l}\text { MEAN } \\
3.174\end{array}$} & \multirow{2}{*}{$\begin{array}{l}\text { VARSPD } \\
4.997\end{array}$} & \multirow{2}{*}{$\begin{array}{l}\text { SDSRD } \\
2.235\end{array}$} & \multirow{2}{*}{$\begin{array}{l}\text { VARVEC } \\
12.818\end{array}$} & \multirow{2}{*}{$\begin{array}{l}\text { SDVEC } \\
3.580\end{array}$} & \multirow{3}{*}{$\begin{array}{l}\mathrm{N} \\
49\end{array}$} \\
\hline 658 & 1 & 224 & 00 & & & & & & \\
\hline & 2 & -5 & & 1.605 & 0.852 & 0.923 & 2.621 & 1.619 & \\
\hline 664 & 1 & 224 & 12 & 2.383 & 4.653 & 2.157 & 7.629 & 2.762 & 36 \\
\hline & 2 & & & 1.875 & 1.279 & 1.131 & 3.144 & 1.773 & 64 \\
\hline & 3 & & & 5.016 & 13.171 & 3.629 & 20.121 & 4.486 & 36 \\
\hline 665 & 1 & 224 & $12 B$ & 4.488 & 4.983 & 2.232 & 7.197 & 2.683 & 36 \\
\hline 672 & 1 & 225 & 00 & 3.773 & 1.630 & 1.277 & 7.305 & 2.703 & 36 \\
\hline & 2 & & & 3.375 & 1.484 & 1.218 & 2.842 & 1.686 & 49 \\
\hline & 3 & & & 2.236 & 1.679 & 1.296 & 5.276 & 2.297 & 36 \\
\hline 678 & 1 & 225 & 12 & 3.562 & 2.524 & 1.589 & 6.573 & 2.564 & 49 \\
\hline 679 & 1 & 225 & $12 \mathrm{~B}$ & 2.149 & 1.781 & 1.335 & 4.512 & 2.124 & 36 \\
\hline & 2 & & & 2.277 & 1.491 & 1.221 & 6.644 & 2.578 & 36 \\
\hline 686 & 1 & 226 & 00 & 3.605 & 2.466 & 1.570 & 11.983 & 3.462 & 49 \\
\hline & 2 & & & 2.185 & 0.515 & 0.718 & 2.304 & 1.518 & 49 \\
\hline 692 & 1 & 226 & 12. & 5.025 & 1.564 & 1.250 & 7.251 & 2.693 & 36 \\
\hline 693 & 1 & $226^{\circ}$ & $12 \mathrm{~B}$ & 2.763 & 1.939 & 1.393 & 9.068 & 3.011 & 49 \\
\hline & 2 & & & 2.426 & 1.560 & 1.249 & 6.236 & 2.497 & 36 \\
\hline & 3 & & & 3.002 & 3.417 & 1.849 & 5.179 & 2.276 & 36 \\
\hline 700 & 1 & 227 & 00 & 2.635 & 2.865 & 1.693 & 8.701 & 2.950 & 100 \\
\hline 701 & 1 & 227 & $O O B$ & 2.737 & 1.420 & 1.192 & 2.391 & 1.546 & 36 \\
\hline & 2 & & & 2.743 & 1.318 & 1.148 & 4.070 & 2.017 & 36 \\
\hline & 3 & & & 2.498 & 0.691 & 0.831 & 3.170 & 1.780 & 36 \\
\hline 708 & 1 & 227 & 12 & 1.511 & 0.565 & 0.751 & 2.900 & 1.703 & 36 \\
\hline & 2 & & & 4.297 & 1.896 & 1.377 & 4.195 & 2.048 & 36 \\
\hline 715 & 1 & 228 & 00 & 4.260 & 6.628 & 2.574 & 24.545 & 4.954 & 49 \\
\hline & 2 & & & 2.741 & 1.226 & 1.107 & 5.751 & 2.398 & 49 \\
\hline & 3 & & & 2.368 & 0.998 & 0.999 & 5.511 & 2.348 & 36 \\
\hline & 4 & & & 3.883 & 6.015 & 2.452 & 20.904 & 4.572 & 49 \\
\hline 716 & 1 & 228 & $O O B$ & 3.018 & 3.563 & 1.888 & 5.888 & 2.427 & 36 \\
\hline 721 & 1 & 228 & 12 & 3.713 & 4.617 & 2.149 & 10.538 & 3.246 & 64 \\
\hline 722 & 1 & 228 & $12 \mathrm{~B}$ & 2.162 & 1.441 & 1.200 & 5.380 & 2.319 & 36 \\
\hline & 2 & & & 6.020 & 4.190 & 2.047 & 14.134 & 3.759 & 49 \\
\hline 729 & 1 & 229 & 00 & 3.125 & 1.688 & 1.299 & 5.847 & 2.418 & 49 \\
\hline & 2 & & & 3.701 & 1.910 & 1.382 & 7.780 & 2.789 & 36 \\
\hline 735 & 1 & 229 & 12 & 2.492 & 1.150 & 1.072 & 5.966 & 2.443 & 36 \\
\hline 736 & 1 & 229 & $12 \mathrm{~B}$ & 2.082 & 0.748 & 0.865 & 2.868 & 1.694 & 49 \\
\hline & 2 & & & 3.765 & 1.702 & 1.304 & 2.705 & 1.645 & 49 \\
\hline & 3 & & & 2.360 & 1.512 & 1.229 & 5.517 & 2.349 & 49 \\
\hline 744 & 1 & 230 & 00 & 5.561 & 8.405 & 2.899 & 28.691 & 5.356 & 121 \\
\hline & 2 & & & 4.216 & 7.305 & 2.703 & 14.400 & 3.795 & 121 \\
\hline & 3 & & & 2.708 & 5.941 & 2.437 & 11.356 & 3.370 & 121 \\
\hline 750 & 1 & 230 & 12 & 2.489 & 1.783 & 1.335 & 6.965 & 2.639 & 121 \\
\hline & 2 & & & 3.630 & 3.119 & 1.766 & 5.756 & 2.399 & 49 \\
\hline & 3 & & & 3.943 & 1.580 & 1.257 & 7.814 & 2.795 & 64 \\
\hline 751 & 1 & 230 & $12 \mathrm{~B}$ & 2.456 & 2.274 & 1.508 & 6.511 & 2.552 & 49 \\
\hline 758 & 1 & 231 & 00 & 3.938 & 2.490 & 1.578 & 15.628 & 3.953 & 49 \\
\hline 772 & 1 & 232 & 00 & 2.428 & 1.183 & 1.088 & 7.059 & 2.657 & 49 \\
\hline 773 & 1 & 232 & $00 \mathrm{~B}$ & 1.701 & 0.912 & 0.955 & 2.848 & 1.688 & 36 \\
\hline 779 & 1 & 232 & 12 & 1.568 & 0.874 & 0.935 & 2.360 & 1.536 & 49 \\
\hline & 2 & & & 1.928 & 0.668 & 0.817 & 3.566 & 1.888 & 49 \\
\hline & 3 & & & 4.159 & 5.672 & 2.382 & 19.459 & 4.411 & 49 \\
\hline 780 & 1 & 232 & $12 \mathrm{~B}$ & 2.938 & 2.065 & 1.437 & 4.608 & 2.147 & 36 \\
\hline 787 & 1 & 233 & 00 & 5.391 & 25.609 & 5.060 & 44.731 & 6.688 & 64 \\
\hline
\end{tabular}




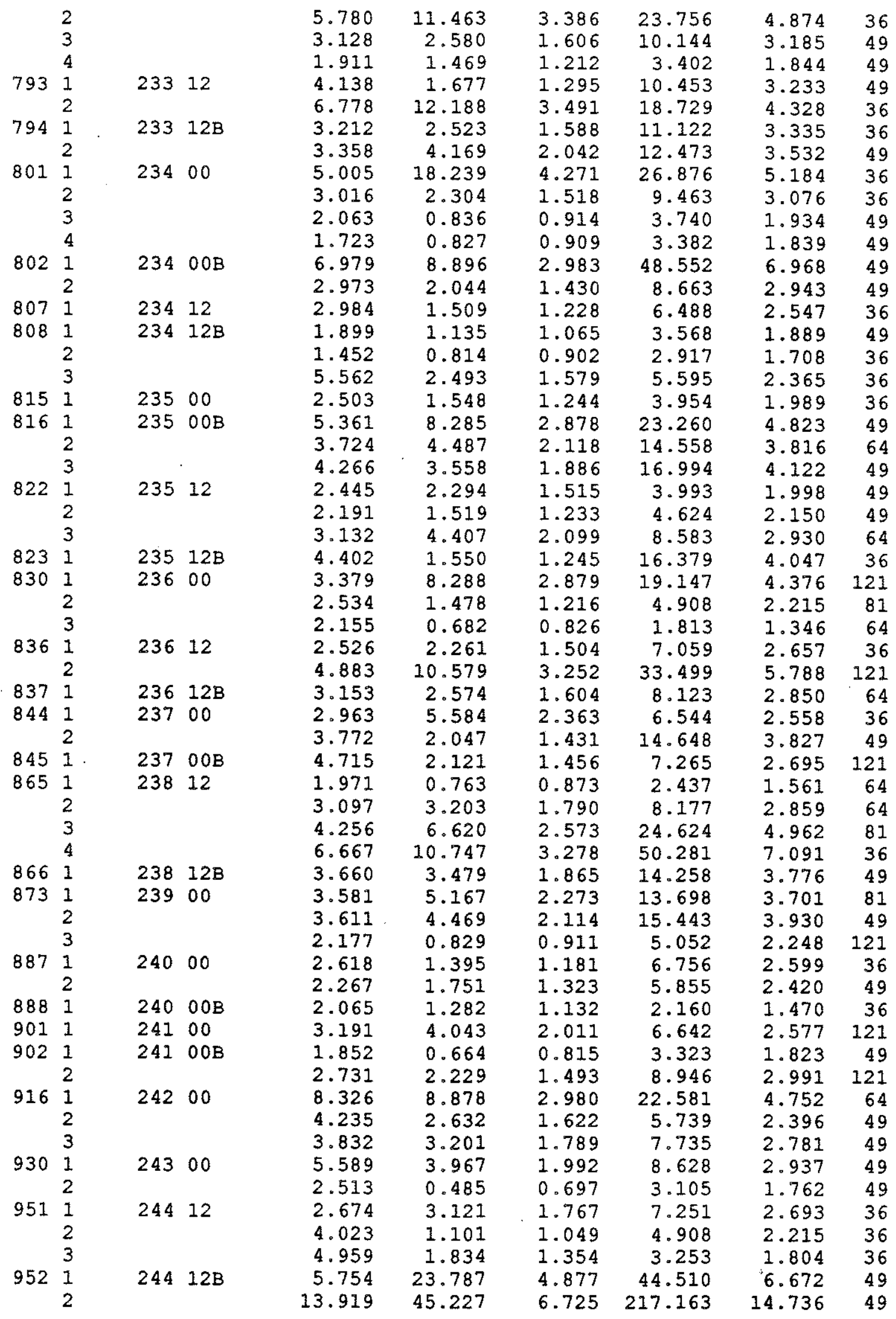




\begin{tabular}{|c|c|c|c|c|c|c|c|c|c|}
\hline \multirow[t]{3}{*}{959} & 1 & 245 & 00 & 3.963 & 2.018 & 1.421 & 3.204 & 1.790 & 49 \\
\hline & 2 & & & 3.074 & 1.069 & 1.034 & 1.489 & 1.220 & 36 \\
\hline & 3 & & & 5.348 & 6.390 & 2.528 & 13.424 & 3.664 & 49 \\
\hline \multirow[t]{3}{*}{973} & 1 & 246 & 00 & 3.948 & 3.405 & 1.845 & 3.663 & 1.914 & 36 \\
\hline & 2 & & & 3.814 & 1.456 & 1.207 & 2.576 & 1.605 & 36 \\
\hline & 3 & & & 2.550 & 1.192 & 1.092 & 4.329 & 2.081 & 49 \\
\hline \multirow{3}{*}{$\begin{array}{l}987 \\
988\end{array}$} & 1 & 247 & 00 & 4.685 & 3.782 & 1.945 & 21.070 & 4.590 & 121 \\
\hline & 1 & 247 & $O O B$ & 11.104 & 66.054 & 8.127 & 160.790 & 12.680 & 36 \\
\hline & 2 & & & 2.013 & 0.838 & 0.916 & 3.695 & 1.922 & 100 \\
\hline \multirow[t]{3}{*}{1002} & 1 & 248 & 00 & 2.766 & 1.986 & 1.409 & 4.890 & 2.211 & 121 \\
\hline & 2 & & & 1.915 & 1.171 & 1.082 & 3.016 & 1.737 & 49 \\
\hline & 3 & & & 2.322 & 0.632 & 0.795 & 4.162 & 2.040 & 121 \\
\hline \multirow{3}{*}{1008} & 1 & 248 & $\mathrm{OOB}$ & 5.714 & 7.380 & 2.717 & 15.843 & 3.980 & 64 \\
\hline & 1 & 248 & 12 & 3.593 & 2.826 & 1.681 & 8.139 & 2.853 & 36 \\
\hline & 2 & & & 10.416 & 54.558 & 7.386 & 142.025 & 11.917 & 121 \\
\hline \multirow[t]{2}{*}{1009} & 1 & 248 & $12 \mathrm{~B}$ & 2.308 & 1.492 & 1.221 & 6.841 & 2.616 & 121 \\
\hline & 2 & & & 2.468 & 1.367 & 1.169 & 4.570 & 2.138 & 121 \\
\hline \multirow[t]{2}{*}{1016} & 1 & 249 & 00 & 2.205 & 1.327 & 1.152 & 4.893 & 2.212 & 49 \\
\hline & 2 & & & 4.866 & 11.635 & 3.411 & 28.446 & 5.334 & 81 \\
\hline 1017 & 1 & 249 & $00 \mathrm{~B}$ & 4.187 & 3.463 & 1.861 & 5.181 & 2.276 & \\
\hline 1030 & 1 & 250 & 00 & 2.471 & 1.139 & 1.067 & 4.759 & 2.182 & 121 \\
\hline \multirow[t]{3}{*}{1031} & 1 & 250 & $O O B$ & 2.389 & 1.035 & 1.017 & 6.215 & 2.493 & 6 \\
\hline & 2 & & & 4.785 & 17.628 & 4.199 & 24.822 & 4.982 & 3 \\
\hline & 3 & & & 3.862 & 0.494 & 0.703 & 4.853 & 2.203 & 3 \\
\hline 1051 & 1 & 251 & 12 & 7.522 & 17.801 & 4.219 & 71.073 & 8.430 & 123 \\
\hline \multirow{2}{*}{1052} & 1 & 251 & $12 \mathrm{~B}$ & 7.679 & 27.876 & 5.280 & 49.299 & 7.021 & 100 \\
\hline & 2 & & & 7.737 & 14.553 & 3.815 & 50.327 & 7.094 & 49 \\
\hline \multirow[t]{2}{*}{1059} & 1 & 252 & 00 & 2.494 & 1.518 & 1.232 & 3.949 & 1.987 & \\
\hline & 2 & & & 3.455 & 1.950 & 1.397 & 6.227 & 2.495 & \\
\hline \multirow{5}{*}{$\begin{array}{l}1060 \\
1074\end{array}$} & 1 & 252 & $O O B$ & 2.137 & 0.808 & 0.899 & 3.017 & 1.737 & 3 \\
\hline & 1 & 253 & 00 & 5.944 & 7.844 & 2.801 & 22.291 & 4.721 & \\
\hline & 2 & & & 9.458 & 25.542 & 5.054 & 114.015 & 10.678 & \\
\hline & 3 & & & 4.626 & 6.251 & 2.500 & 26.758 & 5.173 & \\
\hline & 4 & & & 2.256 & 1.827 & 1.352 & 6.833 & 2.614 & 3 \\
\hline
\end{tabular}


Table 20: Atlas-FNOC difference field vector statistics - 1-day averages

\begin{tabular}{|c|c|c|c|c|c|c|c|}
\hline DAY & REVS & MEAN & VARSPD & SDSPD & VARVEC & SDVEC & $\mathrm{N}$ \\
\hline 224 & $658-672$ & 3.024 & 4.714 & 2.171 & 12.024 & 3.468 & 378 \\
\hline 225 & $672-686$ & 2.939 & 2.139 & 1.462 & 9.486 & 3.080 & 340 \\
\hline 226 & $686-701$ & 2.907 & 2.391 & 1.546 & 10.734 & 3.276 & 463 \\
\hline 227 & $700-716$ & 2.980 & 3.245 & 1.801 & 11.714 & 3.423 & 499 \\
\hline 228 & $715-729$ & 3.585 & 4.463 & 2.113 & 16.022 & 4.003 & 453 \\
\hline 229 & $729-744$ & 3.627 & 6.077 & 2.465 & 17.008 & 4.124 & 631 \\
\hline 230 & $744-758$ & 3.677 & 5.936 & 2.436 & 17.109 & 4.136 & 695 \\
\hline 231 & $758-773$ & 2.785 & 2.421 & 1.556 & 9.590 & 3.097 & 134 \\
\hline 232 & $772-787$ & 3.135 & 7.978 & 2.825 & 17.030 & 4.127 & 466 \\
\hline 233 & $787-802$ & 3.913 & 9.652 & 3.107 & 22.993 & 4.795 & 636 \\
\hline 234 & $801-816$ & 3.555 & 6.558 & 2.561 & 17.722 & 4.210 & 623 \\
\hline 235 & $815-830$ & 3.230 & 4.742 & 2.178 & 15.095 & 3.885 & 662 \\
\hline 236 & $830-845$ & 3.604 & 5.586 & 2.364 & 18.435 & 4.294 & 693 \\
\hline 237 & $844-845$ & 4.184 & 3.143 & 1.773 & 12.387 & 3.520 & 206 \\
\hline 238 & $865-873$ & 3.337 & 5.178 & 2.276 & 15.878 & 3.985 & 545 \\
\hline 239 & $87.3-888$ & 2.715 & 2.873 & 1.695 & 9.685 & 3.112 & 372 \\
\hline 240 & $887-902$ & 2.638 & 2.553 & 1.598 & 8.974 & 2.996 & 412 \\
\hline 241 & $901-916$ & 3.831 & 7.329 & 2.707 & 21.719 & 4.660 & 453 \\
\hline 242 & $916-930$ & 5.097 & 8.395 & 2.897 & 27.736 & 5.267 & 260 \\
\hline 243 & 930 & 4.051 & 4.593 & 2.143 & 10.179 & 3.190 & 98 \\
\hline 244 & $951-959$ & 5.737 & 23.976 & 4.896 & 55.739 & 7.466 & 340 \\
\hline 245 & $959-973$ & 3.809 & 3.478 & 1.865 & 10.017 & 3.165 & 255 \\
\hline 246 & $973-988$ & 4.159 & 14.428 & 3.798 & 30.487 & 5.522 & 378 \\
\hline 247 & $987-1003$ & 3.665 & 11.145 & 3.338 & 22.508 & 4.744 & 612 \\
\hline 248 & $1002-1017$ & 4.017 & 17.087 & 4.134 & 32.481 & 5.699 & 920 \\
\hline 249 & $1016-1031$ & 3.348 & 5.838 & 2.416 & 16.813 & 4.100 & 423 \\
\hline 250 & $1030-1031$ & 2.969 & 4.044 & 2.011 & 11.189 & 3.345 & 257 \\
\hline 251 & $1051-1060$ & 6.004 & 19.742 & 4.443 & 53.889 & 7.341 & 404 \\
\hline 252 & $1059-1074$ & 4.514 & 12.980 & 3.603 & 31.492 & 5.612 & 317 \\
\hline
\end{tabular}


Table 21: Atlas-FNOC difference field vector statistics 1-day/10 deg latitude averages

\begin{tabular}{|c|c|c|c|c|c|c|c|}
\hline DAY & LAT & MEAN & VARSPD & SDSPD & VARVEC & SDVEC & $\mathrm{N}$ \\
\hline \multirow[t]{3}{*}{224} & $20-30$ & 2.000 & 2.023 & 1.422 & 5.198 & 2.280 & 172 \\
\hline & $30-40$ & 3.900 & 6.173 & 2.485 & 13.878 & 3.725 & 170 \\
\hline & $40-50$ & 3.773 & 1.630 & 1.277 & 7.305 & 2.703 & 36 \\
\hline \multirow[t]{2}{*}{225} & $20-30$ & 2.209 & 1.272 & 1.128 & 5.321 & 2.307 & 157 \\
\hline & $30-40$ & 3.271 & 2.246 & 1.499 & 12.536 & 3.541 & 183 \\
\hline \multirow[t]{3}{*}{226} & $20-30$ & 2.547 & 1.868 & 1.367 & 8.021 & 2.832 & 234 \\
\hline & $30-40$ & 2.950 & 2.201 & 1.484 & 10.416 & 3.227 & 193 \\
\hline & $40-50$ & 5.025 & 1.564 & 1.250 & 7.251 & 2.693 & 36 \\
\hline \multirow[t]{3}{*}{227} & $20-30$ & 2.659 & 3.032 & 1.741 & 9.803 & 3.131 & 257 \\
\hline & $30-40$ & 3.083 & 2.152 & 1.467 & 8.620 & 2.936 & 193 \\
\hline & $40-50$ & 4.260 & 6.628 & 2.574 & 24.545 & 4.954 & 49 \\
\hline \multirow[t]{3}{*}{228} & $20-30$ & 3.665 & 4.541 & 2.131 & 16.138 & 4.017 & 255 \\
\hline & $30-40$ & 3.917 & 5.035 & 2.244 & 14.172 & 3.765 & 198 \\
\hline & $40-50$ & 4.260 & 6.628 & 2.574 & 24.545 & 4.954 & 49 \\
\hline \multirow[t]{3}{*}{229} & $20-30$ & 2.808 & 3.769 & 1.941 & 10.186 & 3.192 & 255 \\
\hline & $30-40$ & 3.700 & 5.281 & 2.298 & 13.609 & 3.689 & 219 \\
\hline & $40-50$ & 4.858 & 8.399 & 2.898 & 26.729 & 5.170 & 157 \\
\hline \multirow[t]{3}{*}{230} & $20-30$ & 2.574 & 3.585 & 1.894 & 10.206 & 3.195 & 291 \\
\hline & $30-40$ & 3.691 & 5.705 & 2.388 & 15.056 & 3.880 & 219 \\
\hline & $40-50$ & 4.779 & 5.927 & 2.435 & 21.468 & 4.633 & 234 \\
\hline \multirow[t]{2}{*}{231} & $30-40$ & 2.120 & 1.187 & 1.089 & 5.344 & 2.312 & 85 \\
\hline & $40-50$ & 3.938 & 2.490 & 1.578 & 15.628 & 3.953 & 49 \\
\hline \multirow[t]{3}{*}{232} & $20-30$ & 2.062 & 1.695 & 1.302 & 3.806 & 1.951 & 134 \\
\hline & $30-40$ & 3.134 & 5.210 & 2.283 & 14.831 & 3.851 & 268 \\
\hline & $40-50$ & 5.531 & 20.384 & 4.515 & 49.366 & 7.026 & 100 \\
\hline \multirow[t]{3}{*}{233} & $20-30$ & 2.401 & 2.037 & 1.427 & 7.804 & 2.794 & 183 \\
\hline & $30-40$ & 4.137 & 8.089 & 2.844 & 23.111 & 4.807 & 353 \\
\hline & $40-50$ & 5.266 & 16.355 & 4.044 & 38.768 & 6.226 & 172 \\
\hline \multirow[t]{3}{*}{234} & $20-30$ & 2.517 & 2.608 & 1.615 & 8.409 & 2.900 & 268 \\
\hline & $30-40$ & 4.335 & 8.582 & 2.929 & 21.418 & 4.628 & 270 \\
\hline & $40-50$ & 3.956 & 5.781 & 2.404 & 15.090 & 3.885 & 121 \\
\hline \multirow[t]{3}{*}{235} & $20-30$ & 3.057 & 2.793 & 1.671 & 11.339 & 3.367 & 234 \\
\hline & $30-40$ & 3.143 & 3.451 & 1.858 & 13.262 & 3.642 & 294 \\
\hline & $40-50$ & 3.726 & 7.888 & 2.809 & 20.867 & 4.568 & 234 \\
\hline \multirow[t]{3}{*}{236} & $20-30$ & 3.674 & 2.877 & 1.696 & 15.000 & 3.873 & 298 \\
\hline & $30-40$ & 3.477 & 3.311 & 1.820 & 14.322 & 3.784 & 338 \\
\hline & $40-50$ & 4.131 & 9.961 & 3.156 & 27.004 & 5.197 & 242 \\
\hline \multirow[t]{2}{*}{237} & $20-30$ & 4.443 & 2.271 & 1.507 & 12.717 & 3.566 & 170 \\
\hline & $30-40$ & 4.313 & 3.430 & 1.852 & 9.064 & 3.011 & 157 \\
\hline \multirow[t]{3}{*}{238} & $20-30$ & 2.431 & 1.760 & 1.327 & 7.605 & 2.758 & 234 \\
\hline & $30-40$ & 4.083 & 6.524 & 2.554 & 22.049 & 4.696 & 279 \\
\hline & $40-50$ & 3.918 & 5.972 & 2.444 & 20.665 & 4.546 & 162 \\
\hline \multirow[t]{3}{*}{239} & $20-30$ & 2.178 & 1.119 & 1.058 & 5.562 & 2.358 & 206 \\
\hline & $30-40$ & 3.190 & 3.379 & 1.838 & 12.516 & 3.538 & 85 \\
\hline & $40-50$ & 3.581 & 5.167 & 2.273 & 13.698 & 3.701 & 81 \\
\hline \multirow[t]{2}{*}{240} & $20-30$ & 2.758 & 2.863 & 1.692 & 9.464 & 3.076 & 327 \\
\hline & $30-40$ & 2.177 & 1.105 & 1.051 & 5.099 & 2.258 & 85 \\
\hline \multirow[t]{3}{*}{241} & $20-30$ & 3.107 & 3.276 & 1.810 & 10.892 & 3.300 & 291 \\
\hline & $30-40$ & 3.043 & 3.064 & 1.751 & 6.737 & 2.596 & 98 \\
\hline & $40-50$ & 6.552 & 10.271 & 3.205 & 41.141 & 6.414 & 113 \\
\hline \multirow[t]{2}{*}{242} & $20-30$ & 3.172 & 2.263 & 1.504 & 6.261 & 2.502 & 98 \\
\hline & $30-40$ & 4.912 & 3.729 & 1.931 & 14.386 & 3.793 & 98 \\
\hline
\end{tabular}




\begin{tabular}{|c|c|c|c|c|c|c|c|}
\hline & $40-50$ & 6.552 & 10.271 & 3.205 & 41.141 & 6.414 & 113 \\
\hline 243 & $20-30$ & 2.513 & 0.485 & 0.697 & 3.105 & 1.762 & 49 \\
\hline & $30-40$ & 5.589 & 3.967 & 1.992 & 8.628 & 2.937 & 49 \\
\hline 244 & $20-30$ & 4.778 & 13.381 & 3.658 & 35.656 & 5.971 & 134 \\
\hline & $30-40$ & 7.748 & 44.986 & 6.707 & 104.129 & 10.204 & 121 \\
\hline & $40-50$ & 4.384 & 2.163 & 1.471 & 11.631 & 3.410 & 85 \\
\hline 245 & $20-30$ & 3.913 & 4.565 & 2.137 & 11.372 & 3.372 & 134 \\
\hline & $30-40$ & 3.511 & 2.399 & 1.549 & 4.932 & 2.221 & 72 \\
\hline & $40-50$ & 3.963 & 2.018 & 1.421 & 3.204 & 1.790 & 49 \\
\hline 246 & $20-30$ & 3.367 & 3.535 & 1.880 & 12.054 & 3.472 & 306 \\
\hline & $30-40$ & 7.526 & 47.225 & 6.872 & 102.856 & 10.142 & 72 \\
\hline 247 & $20-30$ & 3.068 & 3.248 & 1.802 & 10.335 & 3.215 & 342 \\
\hline & $30-40$ & 5.767 & 30.984 & 5.566 & 62.918 & 7.932 & 149 \\
\hline & $40-50$ & 2.766 & 1.986 & 1.409 & 4.890 & 2.211 & 121 \\
\hline 248 & $20-30$ & 3.078 & 4.892 & 2.212 & 13.969 & 3.738 & 359 \\
\hline & $30-40$ & 3.229 & 4.543 & 2.131 & 13.801 & 3.715 & 355 \\
\hline & $40-50$ & 6.591 & 42.845 & 6.546 & 78.280 & 8.848 & 242 \\
\hline 249 & $20-30$ & 3.726 & 7.014 & 2.648 & 20.504 & 4.528 & 310 \\
\hline & $30-40$ & 2.763 & 2.347 & 1.532 & 9.253 & 3.042 & 14 \\
\hline 250 & $20-30$ & 3.162 & 4.903 & 2.214 & 12.450 & 3.528 & 193 \\
\hline & $30-40$ & 2.389 & 1.035 & 1.017 & 6.215 & 2.493 & 64 \\
\hline 251 & $20-30$ & 5.482 & 21.568 & 4.644 & 38.062 & 6.169 & 185 \\
\hline & $30-40$ & 5.115 & 14.896 & 3.859 & 38.601 & 6.213 & 98 \\
\hline & $40-50$ & 7.522 & 17.801 & 4.219 & 71.073 & 8.430 & 121 \\
\hline 50 & $20-30$ & 2.706 & 1.935 & 1.391 & 9.006 & 3.001 & 121 \\
\hline & $30-40$ & 5.526 & 19.497 & 4.416 & 48.866 & 6.990 & 147 \\
\hline & $40-50$ & 5.944 & 7.844 & 2.801 & 22.291 & 4.721 & 49 \\
\hline
\end{tabular}


Table 22: Atlas SASS wind stress curl - individual area average

\begin{tabular}{|c|c|c|c|c|c|c|}
\hline \multicolumn{2}{|c|}{ REV/BOX } & MEAN & VARIANCE & WVAR & STND DEV & $\mathrm{N}$ \\
\hline \multirow[t]{2}{*}{658} & 1 & $-0.79 E-08$ & $0.15 \mathrm{E}-13$ & 1.04 & $0.12 \mathrm{E}-06$ & 25 \\
\hline & 2 & $0.72 \mathrm{E}-07$ & $0.40 \mathrm{E}-14$ & 0.44 & $0.63 E-07$ & 16 \\
\hline \multirow[t]{3}{*}{664} & 1 & $0.25 \mathrm{E}-07$ & $0.50 E-14$ & 0.94 & $0.71 E-07$ & 16 \\
\hline & 2 & $0.22 \mathrm{E}-07$ & $0.17 \mathrm{E}-14$ & 0.79 & $0.41 E-07$ & 36 \\
\hline & 3 & $0.49 E-06$ & $0.96 \mathrm{E}-13$ & 0.29 & $0.31 E-06$ & 16 \\
\hline 665 & 1 & $0.55 \mathrm{E}-10$ & $0.79 \mathrm{E}-14$ & 1.07 & $0.89 \mathrm{E}-07$ & 16 \\
\hline \multirow[t]{3}{*}{672} & 1 & $0.76 \mathrm{E}-07$ & $0.67 \mathrm{E}-13$ & 0.98 & $0.26 E-06$ & 16 \\
\hline & 2 & $0.77 \mathrm{E}-07$ & $0.23 E-13$ & 0.82 & $0.15 E-06$ & 25 \\
\hline & 3 & $0.95 E-08$ & $0.27 \mathrm{E}-15$ & 0.79 & $0.16 \mathrm{E}-07$ & 16 \\
\hline 678 & 1 & $-0.32 E-07$ & $0.28 \mathrm{E}-14$ & 0.75 & $0.53 \mathrm{E}-07$ & 25 \\
\hline \multirow[t]{2}{*}{679} & 1 & $0.68 E-07$ & $0.56 \mathrm{E}-14$ & 0.57 & $0.75 \mathrm{E}-07$ & 16 \\
\hline & 2 & $0.99 \mathrm{E}-08$ & $0.34 \mathrm{E}-14$ & 1.03 & $0.58 \mathrm{E}-07$ & 16 \\
\hline \multirow[t]{2}{*}{686} & 1 & $-0.48 E-07$ & $0.63 \mathrm{E}-14$ & 0.75 & $0.79 E-07$ & 25 \\
\hline & 2 & $0.60 \mathrm{E}-07$ & $0.51 E-14$ & 0.60 & $0.71 \mathrm{E}-07$ & 25 \\
\hline & $\overline{1}$ & $0.24 \mathrm{E}-07$ & $0.58 E-14$ & 0.96 & $0.76 \mathrm{E}-07$ & 16 \\
\hline \multirow[t]{3}{*}{693} & 1 & $0.12 \mathrm{E}-06$ & $0.72 E-14$ & 0.34 & $0.85 E-07$ & 25 \\
\hline & 2 & $0.48 \mathrm{E}-07$ & $0.64 \mathrm{E}-15$ & 0.22 & $0.25 \mathrm{E}-07$ & 16 \\
\hline & 3 & $0.58 E-0.9$ & $0.56 \mathrm{E}-13$ & 1.07 & $0.24 \mathrm{E}-06$ & 16 \\
\hline 700 & 1 & $0.32 \mathrm{E}-07$ & $0.97 \mathrm{E}-14$ & 0.92 & $0.99 E-07$ & 64 \\
\hline \multirow[t]{3}{*}{701} & 1 & $-0.10 \mathrm{E}-06$ & $0.21 E-14$ & 0.17 & $0.45 E-07$ & 16 \\
\hline & 2 & $0.82 E-07$ & $0.31 E-14$ & 0.32 & $0.56 \mathrm{E}-07$ & 16 \\
\hline & 3 & $0.23 E-06$ & $0.83 E-14$ & 0.14 & $0.91 E-07$ & 16 \\
\hline \multirow{2}{*}{708} & 1 & $0.20 \mathrm{E}-06$ & $0.16 \mathrm{E}-13$ & 0.28 & $0.13 E-06$ & 16 \\
\hline & 2 & $0.48 E-07$ & $0.13 E-13$ & 0.89 & $0.11 E-06$ & 16 \\
\hline \multirow[t]{4}{*}{715} & 1 & $0.11 E-05$ & $0.51 E-12$ & 0.31 & $0.71 \mathrm{E}-06$ & 25 \\
\hline & 2 & $0.88 E-07$ & $0.57 E-13$ & 0.91 & $0.24 E-06$ & 25 \\
\hline & 3 & $0.99 E-07$ & $0.57 \mathrm{E}-14$ & 0.37 & $0.75 E-07$ & 16 \\
\hline & 4 & $0.21 E-06$ & $0.98 E-13$ & 0.71 & $0.31 E-06$ & 25 \\
\hline 716 & 1 & $0.35 E-07$ & $0.14 \mathrm{E}-14$ & 0.55 & $0.38 E-07$ & 16 \\
\hline 721 & 1 & $-0.74 E-07$ & $0.46 E-13$ & 0.92 & $0.21 E-06$ & 36 \\
\hline \multirow[t]{2}{*}{722} & 1 & $0.25 E-06$ & $0.11 \mathrm{E}-13$ & 0.15 & $0.10 E-06$ & 16 \\
\hline & 2 & $0.68 E-07$ & $0.94 \mathrm{E}-13$ & 0.99 & $0.31 E-06$ & 25 \\
\hline \multirow[t]{2}{*}{729} & 1 & $0.74 E-07$ & $0.49 \mathrm{E}-14$ & 0.48 & $0.70 E-07$ & 25 \\
\hline & 2 & $0.88 \mathrm{E}-07$ & $0.75 E-14$ & 0.51 & $0.87 E-07$ & 16 \\
\hline 735 & 1 & $-0.46 \mathrm{E}-06$ & $0.43 E-13$ & 0.17 & $0.21 E-06$ & 16 \\
\hline \multirow{3}{*}{736} & 1 & $0.10 \mathrm{E}-06$ & $0.32 E-14$ & 0.23 & $0.56 E-07$ & 25 \\
\hline & 2 & $-0.53 E-07$ & $0.28 E-14$ & 0.52 & $0.53 E-07$ & 25 \\
\hline & 3 & $0.87 E-07$ & $0.58 E-14$ & 0.44 & $0.76 E-07$ & 25 \\
\hline \multirow[t]{3}{*}{744} & 1 & $-0.20 \mathrm{E}-08$ & $0.33 \mathrm{E}-13$ & 1.01 & $0.18 E-06$ & 81 \\
\hline & 2 & $-0.72 E-07$ & $0.52 \mathrm{E}-14$ & 0.50 & $0.72 \mathrm{E}-07$ & 81 \\
\hline & 3 & $0.11 E-06$ & $0.68 E-14$ & 0.36 & $0.83 E-07$ & 81 \\
\hline \multirow[t]{3}{*}{750} & 1 & $0.71 \mathrm{E}-07$ & $0.21 E-13$ & 0.81 & $0.14 \mathrm{E}-06$ & 81 \\
\hline & 2 & $-0.29 \mathrm{E}-07$ & $0.26 \mathrm{E}-14$ & 0.78 & $0.51 \mathrm{E}-07$ & 25 \\
\hline & 3 & $0.13 E-08$ & $0.42 E-14$ & 1.03 & $0.65 \mathrm{E}-07$ & 36 \\
\hline 751 & 1 & $0.11 E-06$ & $0.22 E-13$ & 0.66 & $0.15 E-06$ & 25 \\
\hline 758 & 1 & $-0.38 E-07$ & $0.73 E-14$ & 0.86 & $0.85 E-07$ & 25 \\
\hline 772 & 1 & $-0.12 E-06$ & $0.96 \mathrm{E}-14$ & 0.41 & $0.98 E-07$ & 25 \\
\hline 773 & 1 & $-0.22 \mathrm{E}-07$ & $0.87 \mathrm{E}-15$ & 0.66 & $0.29 \mathrm{E}-07$ & 16 \\
\hline 779 & 1 & $0.37 E-07$ & $0.38 E-14$ & 0.76 & $0.61 E-07$ & 25 \\
\hline & 2 & $0.11 E-06$ & $0.80 E-14$ & 0.42 & $0.90 E-07$ & 25 \\
\hline & 3 & $0.15 E-06$ & $0.59 \mathrm{E}-13$ & 0.73 & $0.24 E-06$ & 25 \\
\hline 780 & 1 & $0.30 \mathrm{E}-06$ & $0.22 E-13$ & 0.21 & $0.15 \mathrm{E}-06$ & 16 \\
\hline 787 & 1 & $-0.48 E-06$ & $0.16 \mathrm{E}-12$ & 0.41 & $0.39 E-06$ & 36 \\
\hline
\end{tabular}




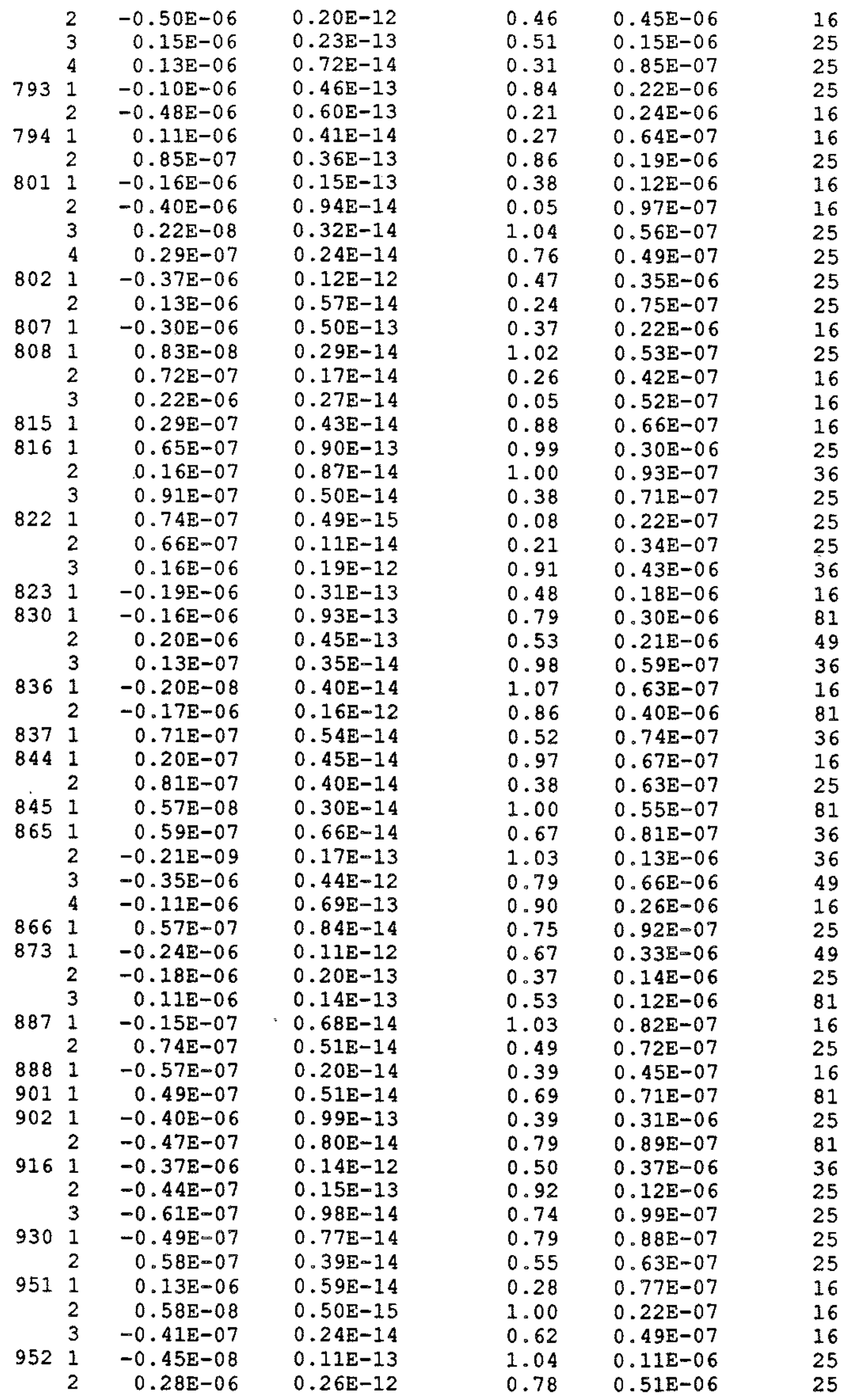




$\begin{array}{rrrrrrr}959 & 1 & -0.14 \mathrm{E}-06 & 0.46 \mathrm{E}-14 & 0.20 & 0.68 \mathrm{E}-07 & 25 \\ & 2 & 0.11 \mathrm{E}-07 & 0.29 \mathrm{E}-15 & 0.73 & 0.17 \mathrm{E}-07 & 16 \\ & 3 & 0.11 \mathrm{E}-06 & 0.60 \mathrm{E}-14 & 0.33 & 0.78 \mathrm{E}-07 & 25 \\ 973 & 1 & 0.66 \mathrm{E}-07 & 0.24 \mathrm{E}-14 & 0.36 & 0.49 \mathrm{E}-07 & 16 \\ & 2 & 0.26 \mathrm{E}-07 & 0.79 \mathrm{E}-15 & 0.56 & 0.28 \mathrm{E}-07 & 16 \\ 9 & 3 & 0.13 \mathrm{E}-06 & 0.16 \mathrm{E}-13 & 0.49 & 0.13 \mathrm{E}-06 & 25 \\ 987 & 1 & 0.11 \mathrm{E}-06 & 0.11 \mathrm{E}-13 & 0.48 & 0.11 \mathrm{E}-06 & 81 \\ 988 & 1 & 0.97 \mathrm{E}-07 & 0.41 \mathrm{E}-11 & 1.06 & 0.20 \mathrm{E}-05 & 16 \\ & 2 & 0.14 \mathrm{E}-06 & 0.72 \mathrm{E}-14 & 0.26 & 0.85 \mathrm{E}-07 & 64 \\ 1002 & 1 & -0.13 \mathrm{E}-06 & 0.76 \mathrm{E}-14 & 0.33 & 0.87 \mathrm{E}-07 & 81 \\ & 2 & 0.14 \mathrm{E}-06 & 0.13 \mathrm{E}-13 & 0.40 & 0.12 \mathrm{E}-06 & 25 \\ & 3 & 0.27 \mathrm{E}-07 & 0.46 \mathrm{E}-14 & 0.87 & 0.68 \mathrm{E}-07 & 81 \\ 1003 & 1 & 0.12 \mathrm{E}-07 & 0.22 \mathrm{E}-13 & 1.02 & 0.15 \mathrm{E}-06 & 36 \\ 1008 & 1 & -0.46 \mathrm{E}-07 & 0.48 \mathrm{E}-14 & 0.73 & 0.69 \mathrm{E}-07 & 16 \\ & 2 & -0.87 \mathrm{E}-07 & 0.46 \mathrm{E}-11 & 1.01 & 0.22 \mathrm{E}-05 & 81 \\ 1009 & 1 & 0.35 \mathrm{E}-07 & 0.70 \mathrm{E}-14 & 0.86 & 0.84 \mathrm{E}-07 & 81 \\ & 2 & 0.60 \mathrm{E}-08 & 0.66 \mathrm{E}-14 & 1.01 & 0.81 \mathrm{E}-07 & 81 \\ 1016 & 1 & 0.10 \mathrm{E}-06 & 0.19 \mathrm{E}-14 & 0.15 & 0.44 \mathrm{E}-07 & 25 \\ & 2 & 0.17 \mathrm{E}-06 & 0.28 \mathrm{E}-12 & 0.92 & 0.53 \mathrm{E}-06 & 49 \\ 1017 & 1 & -0.11 \mathrm{E}-07 & 0.61 \mathrm{E}-14 & 1.04 & 0.78 \mathrm{E}-07 & 16 \\ 1030 & 1 & 0.52 \mathrm{E}-07 & 0.75 \mathrm{E}-14 & 0.74 & 0.87 \mathrm{E}-07 & 81 \\ 1031 & 1 & 0.18 \mathrm{E}-06 & 0.11 \mathrm{E}-13 & 0.27 & 0.11 \mathrm{E}-06 & 36 \\ & 2 & -0.59 \mathrm{E}-07 & 0.13 \mathrm{E}-13 & 0.83 & 0.11 \mathrm{E}-06 & 16 \\ & 3 & -0.12 \mathrm{E}-06 & 0.33 \mathrm{E}-13 & 0.73 & 0.18 \mathrm{E}-06 & 16 \\ 1051 & 1 & 0.14 \mathrm{E}-06 & 0.13 \mathrm{E}-11 & 1.00 & 0.12 \mathrm{E}-05 & 81 \\ 1052 & 1 & 0.19 \mathrm{E}-06 & 0.18 \mathrm{E}-13 & 0.35 & 0.14 \mathrm{E}-06 & 64 \\ & 2 & -0.18 \mathrm{E}-06 & 0.13 \mathrm{E}-12 & 0.83 & 0.36 \mathrm{E}-06 & 25 \\ 1059 & 1 & 0.29 \mathrm{E}-06 & 0.30 \mathrm{E}-13 & 0.27 & 0.17 \mathrm{E}-06 & 25 \\ & 2 & -0.44 \mathrm{E}-07 & 0.80 \mathrm{E}-14 & 0.83 & 0.90 \mathrm{E}-07 & 25 \\ 1060 & 1 & 0.20 \mathrm{E}-07 & 0.26 \mathrm{E}-14 & 0.92 & 0.51 \mathrm{E}-07 & 16 \\ 1074 & 1 & 0.51 \mathrm{E}-06 & 0.85 \mathrm{E}-12 & 0.79 & 0.92 \mathrm{E}-06 & 25 \\ & 2 & 0.23 \mathrm{E}-06 & 0.71 \mathrm{E}-12 & 0.97 & 0.84 \mathrm{E}-06 & 25 \\ & 3 & 0.45 \mathrm{E}-06 & 0.84 \mathrm{E}-13 & 0.30 & 0.29 \mathrm{E}-06 & 25 \\ & 4 & 0.72 \mathrm{E}-07 & 0.18 \mathrm{E}-14 & 0.26 & 0.42 \mathrm{E}-07 & 16 \\ & & & & & & \end{array}$


Table 23: FNOC wind stress curl - individual area averages

\begin{tabular}{|c|c|c|c|c|c|c|}
\hline REV & BOX & MEAN & VARIANCE & WVAR & STND DEV & $\mathrm{N}$ \\
\hline 658 & 1 & $-0.10 E-07$ & $0.82 \mathrm{E}-14$ & 1.03 & $0.91 \mathrm{E}-07$ & 25 \\
\hline & 2 & $0.11 E-07$ & $0.64 \mathrm{E}-15$ & 0.88 & $0.25 E-07$ & 16 \\
\hline 664 & 1 & $0.32 \mathrm{E}-07$ & $0.45 E-14$ & 0.86 & $0.67 E-07$ & 16 \\
\hline & 2 & $0.42 \mathrm{E}-07$ & $0.78 E-15$ & 0.31 & $0.28 E-07$ & 36 \\
\hline & 3 & $0.83 E-07$ & $0.35 \mathrm{E}-14$ & 0.35 & $0.59 \mathrm{E}-07$ & 16 \\
\hline 665 & 1 & $-0.30 \mathrm{E}-07$ & $0.53 \mathrm{E}-15$ & 0.38 & $0.23 E-07$ & 16 \\
\hline 672 & 1 & $0.21 \mathrm{E}-06$ & $0.37 \mathrm{E}-14$ & 0.08 & $0.61 E-07$ & 16 \\
\hline & 2 & $-0.31 E-08$ & $0.51 E-15$ & 1.02 & $0.23 \mathrm{E}-07$ & 25 \\
\hline & 3 & $0.48 \mathrm{E}-07$ & $0.61 E-16$ & 0.03 & $0.78 \mathrm{E}-08$ & 16 \\
\hline 678 & 1 & $0.36 \mathrm{E}-07$ & $0.16 \mathrm{E}-14$ & 0.57 & $0.40 \mathrm{E}-07$ & 25 \\
\hline 679 & 1 & $0.50 \mathrm{E}-08$ & $0.75 \mathrm{E}-14$ & 1.06 & $0.87 E-07$ & 16 \\
\hline & 2 & $0.12 \mathrm{E}-06$ & $0.31 E-14$ & 0.19 & $0.56 E-07$ & 16 \\
\hline 686 & 1 & $0.46 \mathrm{E}-07$ & $0.35 E-14$ & 0.64 & $0.59 \mathrm{E}-07$ & 25 \\
\hline & 2 & $0.37 \mathrm{E}-07$ & $0.39 E-14$ & 0.77 & $0.63 E-07$ & 25 \\
\hline 692 & 1 & $0.23 E-07$ & $0.30 \mathrm{E}-13$ & 1.05 & $0.17 E-06$ & 16 \\
\hline 693 & 1 & $0.12 \mathrm{E}-06$ & $0.13 E-13$ & 0.50 & $0.12 E-06$ & 25 \\
\hline & 2 & $0.24 \mathrm{E}-08$ & $0.15 \mathrm{E}-14$ & 1.06 & $0.38 E-07$ & 16 \\
\hline & 3 & $0.54 E-07$ & $0.78 E-14$ & 0.76 & $0.88 \mathrm{E}-07$ & 16 \\
\hline 700 & 1 & $0.32 \mathrm{E}-07$ & $0.83 \mathrm{E}-14$ & 0.90 & $0.91 E-07$ & 64 \\
\hline 701 & 1 & $-0.70 E-08$ & $0.29 E-15$ & 0.90 & $0.17 \mathrm{E}-07$ & 16 \\
\hline & 2 & $0.19 \mathrm{E}-07$ & $0.27 E-15$ & 0.43 & $0.16 E-07$ & 16 \\
\hline & 3 & $0.13 E-06$ & $0.80 E-15$ & 0.04 & $0.28 E-07$ & 16 \\
\hline 708 & 1 & $0.15 \mathrm{E}-06$ & $0.36 \mathrm{E}-14$ & 0.14 & $0.60 \mathrm{E}-07$ & 16 \\
\hline & 2 & $-0.44 E-08$ & $0.27 E-16$ & 0.61 & $0.52 \mathrm{E}-08$ & 16 \\
\hline 715 & 1 & $0.66 \mathrm{E}-06$ & $0.11 E-12$ & 0.21 & $0.34 E-06$ & 25 \\
\hline & 2 & $0.79 \mathrm{E}-07$ & $0.11 E-13$ & 0.66 & $0.11 E-06$ & 25 \\
\hline & 3 & $0.11 \mathrm{E}-0.6$ & $0.38 \mathrm{E}-14$ & 0.24 & $0.62 \mathrm{E}-07$ & 16 \\
\hline & 4 & $0.11 E-06$ & $0.23 \mathrm{E}-14$ & 0.15 & $0.48 \mathrm{E}-07$ & 25 \\
\hline 716 & 1 & $-0.17 \mathrm{E}-07$ & $0.28 \mathrm{E}-16$ & 0.09 & $0.53 \mathrm{E}-08$ & 16 \\
\hline 721 & 1 & $-0.49 E-07$ & $0.18 \mathrm{E}-14$ & 0.44 & $0.43 E-07$ & 36 \\
\hline 722 & 1 & $0.16 E-06$ & $0.11 E-14$ & 0.04 & $0.33 \mathrm{E}-07$ & 16 \\
\hline & 2 & $0.45 E-07$ & $0.69 \mathrm{E}-15$ & 0.26 & $0.26 \mathrm{E}-07$ & 25 \\
\hline 729 & 1 & $0.45 \mathrm{E}-07$ & $0.69 E-15$ & 0.26 & $0.26 E-07$ & 25 \\
\hline & 2 & $0.11 \mathrm{E}-06$ & $0.25 E-14$ & 0.18 & $0.50 E-07$ & 16 \\
\hline 735 & 1 & $-0.39 E-06$ & $0.46 \mathrm{E}-13$ & 0.23 & $0.21 E-06$ & 16 \\
\hline 736 & 1 & $0.59 \mathrm{E}-07$ & $0.27 \mathrm{E}-14$ & 0.44 & $0.52 E-07$ & 25 \\
\hline & 2 & $-0.33 E-07$ & $0.82 \mathrm{E}-15$ & 0.43 & $0.29 \mathrm{E}-07$ & 25 \\
\hline & 3 & $0.34 \mathrm{E}-09$ & $0.12 \mathrm{E}-14$ & 1.04 & $0.35 E-07$ & 25 \\
\hline 744 & 1 & $-0.19 E-06$ & $0.32 E-13$ & 0.46 & $0.18 E-06$ & 81 \\
\hline & 2 & $0.61 \mathrm{E}-07$ & $0.55 E-14$ & 0.60 & $0.74 \mathrm{E}-07$ & 81 \\
\hline & 3 & $0.80 E-07$ & $0.43 E-14$ & 0.40 & $0.65 E-07$ & 81 \\
\hline 750 & 1 & $0.83 \mathrm{E}-07$ & $0.11 \mathrm{E}-13$ & 0.61 & $0.10 \mathrm{E}-06$ & 81 \\
\hline & 2 & $-0.15 E-07$ & $0.17 \mathrm{E}-15$ & 0.44 & $0.13 \mathrm{E}-07$ & 25 \\
\hline & 3 & $0.32 E-07$ & $0.66 \mathrm{E}-14$ & 0.89 & $0.81 E-07$ & 36 \\
\hline 751 & 1 & $0.60 E-07$ & $0.35 \mathrm{E}-14$ & 0.51 & $0.59 E-07$ & 25 \\
\hline 758 & 1 & $0.44 \mathrm{E}-07$ & $0.87 \mathrm{E}-14$ & 0.84 & $0.93 E-07$ & 25 \\
\hline 772 & 1 & $0.98 E-08$ & $0.22 E-14$ & 1.00 & $0.47 \mathrm{E}-07$ & 25 \\
\hline 773 & 1 & $-0.37 E-07$ & $0.61 E-15$ & 0.31 & $0.25 E-07$ & 16 \\
\hline 779 & 1 & $0.43 E-07$ & $0.12 \mathrm{E}-14$ & 0.41 & $0.35 \mathrm{E}-07$ & 25 \\
\hline & 2 & $0.87 \mathrm{E}-07$ & $0.37 \mathrm{E}-14$ & 0.33 & $0.61 E-07$ & 25 \\
\hline & 3 & $-0.40 \mathrm{E}-07$ & $0.26 \mathrm{E}-13$ & 0.98 & $0.16 \mathrm{E}-06$ & 25 \\
\hline 780 & 1 & $0.14 E-06$ & $0.62 \mathrm{E}-15$ & 0.03 & $0.25 E-07$ & 16 \\
\hline 787 & 1 & $-0.24 E-06$ & $0.57 \mathrm{E}-13$ & 0.50 & $0.24 \mathrm{E}-06$ & 36 \\
\hline
\end{tabular}




\begin{tabular}{|c|c|c|c|c|c|c|}
\hline & 2 & $0.91 \mathrm{E}-09$ & $0.64 \mathrm{E}-14$ & 1.07 & $0.80 \mathrm{E}-07$ & 16 \\
\hline & 3 & $0.11 \mathrm{E}-06$ & $0.11 \mathrm{E}-13$ & 0.47 & $0.10 \mathrm{E}-06$ & 25 \\
\hline & 4 & $0.89 \mathrm{E}-07$ & $0.13 \mathrm{E}-14$ & 0.14 & $0.36 E-07$ & 25 \\
\hline \multirow[t]{2}{*}{793} & 1 & $0.89 \mathrm{E}-07$ & $0.12 \mathrm{E}-13$ & 0.62 & $0.11 E-06$ & 25 \\
\hline & 2 & $-0.20 \mathrm{E}-06$ & $0.26 \mathrm{E}-13$ & 0.39 & $0.16 \mathrm{E}-06$ & 16 \\
\hline \multirow[t]{2}{*}{794} & 1 & $0.81 \mathrm{E}-07$ & $0.46 E-15$ & 0.07 & $0.21 \mathrm{E}-07$ & 16 \\
\hline & 2 & $0.90 E-07$ & $0.72 \mathrm{E}-13$ & 0.93 & $0.27 \mathrm{E}-06$ & 25 \\
\hline \multirow[t]{4}{*}{801} & 1 & $-0.13 \varepsilon-06$ & $0.46 \mathrm{E}-13$ & 0.76 & $0.22 \mathrm{E}-06$ & 16 \\
\hline & 2 & $-0.35 E-06$ & $0.10 \mathrm{E}-13$ & 0.07 & $0.10 \mathrm{E}-06$ & 16 \\
\hline & 3 & $0.90 \mathrm{E}-08$ & $0.22 E-14$ & 1.00 & $0.47 \mathrm{E}-07$ & 25 \\
\hline & 4 & $0.76 \mathrm{E}-07$ & $0.40 E-14$ & 0.42 & $0.63 \mathrm{E}-07$ & 25 \\
\hline \multirow{2}{*}{802} & 1 & $-0.37 \mathrm{E}-07$ & $0.46 \mathrm{E}-13$ & 1.01 & $0.21 E-06$ & 25 \\
\hline & 2 & $0.49 \mathrm{E}-07$ & $0.11 E-14$ & 0.32 & $0.33 E-07$ & 25 \\
\hline 807 & 1 & $-0.21 E-06$ & $0.13 \mathrm{E}-13$ & 0.24 & $0.12 \mathrm{E}-06$ & 16 \\
\hline \multirow[t]{3}{*}{808} & 1 & $0.54 \mathrm{E}-07$ & $0.21 E-14$ & 0.43 & $0.46 E-07$ & 25 \\
\hline & 2 & $0.70 \mathrm{E}-07$ & $0.84 \mathrm{E}-15$ & 0.15 & $0.29 E-07$ & 16 \\
\hline & 3 & $0.88 E-07$ & $0.37 E-14$ & 0.33 & $0.61 E-07$ & 16 \\
\hline 815 & 1 & $0.60 \mathrm{E}-07$ & $0.44 E-15$ & 0.11 & $0.21 E-07$ & 16 \\
\hline \multirow[t]{3}{*}{816} & 1 & $-0.11 E-06$ & $0.20 \mathrm{E}-13$ & 0.63 & $0.14 \mathrm{E}-06$ & 25 \\
\hline & 2 & $0.15 \mathrm{E}-07$ & $0.76 E-14$ & 1.00 & $0.87 E-07$ & 36 \\
\hline & 3 & $0.76 \mathrm{E}-07$ & $0.17 E-14$ & 0.23 & $0.42 \mathrm{E}-07$ & 25 \\
\hline \multirow[t]{3}{*}{822} & 1 & $0.55 E-07$ & $0.67 E-15$ & 0.18 & $0.26 E-07$ & 25 \\
\hline & 2 & $0.47 \mathrm{E}-07$ & $0.11 E-14$ & 0.33 & $0.33 E-07$ & 25 \\
\hline & 3 & $0.25 E-07$ & $0.35 E-13$ & 1.01 & $0.19 \mathrm{E}-06$ & 36 \\
\hline 823 & 1 & $0.12 \mathrm{E}-07$ & $0.62 \mathrm{E}-15$ & 0.86 & $0.25 E-07$ & 16 \\
\hline \multirow[t]{3}{*}{830} & 1 & $-0.14 E-06$ & $0.38 E-13$ & 0.67 & $0.19 \mathrm{E}-06$ & 81 \\
\hline & 2 & $0.21 E-06$ & $0.25 \mathrm{E}-13$ & 0.36 & $0.16 E-06$ & 49 \\
\hline & 3 & $0.72 E-08$ & $0.75 E-15$ & 0.96 & $0.27 E-07$ & 36 \\
\hline \multirow[t]{2}{*}{836} & 1 & $0.98 \mathrm{E}-07$ & $0.55 E-14$ & 0.37 & $0.74 E-07$ & 16 \\
\hline & 2 & $-0.12 E-06$ & $0.12 \mathrm{E}-12$ & 0.91 & $0.35 E-06$ & 81 \\
\hline 837 & 1 & $0.15 E-07$ & $0.80 \mathrm{E}-15$ & 0.81 & $0.28 \mathrm{E}-07$ & 36 \\
\hline \multirow[t]{2}{*}{844} & 1 & $0.49 \mathrm{E}-07$ & $0.46 \mathrm{E}-14$ & 0.68 & $0.68 E-07$ & 16 \\
\hline & 2 & $0.40 E-07$ & $0.14 \mathrm{E}-14$ & 0.49 & $0.38 \mathrm{E}-07$ & 25 \\
\hline 845 & 1 & $0.11 E-07$ & $0.47 \mathrm{E}-15$ & 0.81 & $0.22 E-07$ & 81 \\
\hline \multirow[t]{4}{*}{865} & 1 & $0.23 E-07$ & $0.12 \mathrm{E}-14$ & 0.72 & $0.35 E-07$ & 36 \\
\hline & 2 & $-0.27 \mathrm{E}-09$ & $0.86 \mathrm{E}-14$ & 1.03 & $0.93 \mathrm{E}-07$ & 36 \\
\hline & 3 & $-0.93 E-07$ & $0.48 \mathrm{E}-13$ & 0.86 & $0.22 \mathrm{E}-06$ & 49 \\
\hline & 4 & $-0.11 E-06$ & $0.36 E-14$ & 0.24 & $0.60 \mathrm{E}-07$ & 16 \\
\hline \multirow{4}{*}{$\begin{array}{l}866 \\
873\end{array}$} & 1 & $0.18 \mathrm{E}-07$ & $0.11 E-14$ & 0.80 & $0.33 E-07$ & 25 \\
\hline & 1 & $-0.32 E-06$ & $0.50 \mathrm{E}-13$ & 0.33 & $0.22 \mathrm{E}-06$ & 49 \\
\hline & 2 & $0.35 \mathrm{E}-07$ & $0.44 \mathrm{E}-14$ & 0.81 & $0.66 E-07$ & 25 \\
\hline & 3 & $0.91 E-07$ & $0.19 E-14$ & 0.19 & $0.44 E-07$ & 81 \\
\hline \multirow[t]{2}{*}{887} & 1 & $-0.78 \mathrm{E}-07$ & $0.67 E-14$ & 0.54 & $0.82 \mathrm{E}-07$ & 16 \\
\hline & 2 & $0.26 \mathrm{E}-07$ & $0.56 E-15$ & 0.46 & $0.24 \mathrm{E}-07$ & 25 \\
\hline \multirow{3}{*}{$\begin{array}{l}88 \\
90 \\
90\end{array}$} & 1 & $-0.11 E-07$ & $0.48 E-15$ & 0.85 & $0.22 E-07$ & 16 \\
\hline & 1 & $0.71 \mathrm{E}-07$ & $0.55 \mathrm{E}-14$ & 0.52 & $0.74 \mathrm{E}-07$ & 81 \\
\hline & 1 & $-0.31 E-06$ & $0.43 \mathrm{E}-13$ & 0.31 & $0.21 E-06$ & 25 \\
\hline & 2 & $-0.42 \mathrm{E}-07$ & $0.40 E-14$ & 0.70 & $0.63 E-07$ & 81 \\
\hline \multirow[t]{3}{*}{916} & 1 & $-0.60 \mathrm{E}-07$ & $0.20 \mathrm{E}-13$ & 0.87 & $0.14 \mathrm{E}-06$ & 36 \\
\hline & 2 & $-0.57 E-07$ & $0.39 \mathrm{E}-14$ & 0.56 & $0.62 \mathrm{E}-07$ & 25 \\
\hline & 3 & $-0.14 \mathrm{E}-07$ & $0.37 E-14$ & 0.99 & $0.61 E-07$ & 25 \\
\hline \multirow[t]{2}{*}{930} & 1 & $-0.32 E-07$ & $0.17 \mathrm{E}-14$ & 0.64 & $0.42 \mathrm{E}-07$ & 25 \\
\hline & 2 & $0.59 \mathrm{E}-07$ & $0.15 E-14$ & 0.31 & $0.39 E-07$ & 25 \\
\hline \multirow[t]{3}{*}{951} & 1 & $0.79 E-07$ & $0.22 \mathrm{E}-14$ & 0.26 & $0.47 E-07$ & 16 \\
\hline & 2 & $0.57 \mathrm{E}-08$ & $0.98 E-16$ & 0.79 & $0.99 \mathrm{E}-08$ & 16 \\
\hline & 3 & $-0.81 E-08$ & $0.69 \mathrm{E}-15$ & 0.97 & $0.26 \mathrm{E}-07$ & 16 \\
\hline 952 & 1 & $0.27 \mathrm{E}-06$ & $0.26 \mathrm{E}-12$ & 0.81 & $0.51 E-06$ & 25 \\
\hline & 2 & $-0.11 E-05$ & $0.28 \mathrm{E}-10$ & 1.00 & $0.53 E-05$ & 25 \\
\hline
\end{tabular}




\begin{tabular}{|c|c|c|c|c|c|c|}
\hline \multirow[t]{2}{*}{959} & 1 & $-0.75 E-07$ & $0.22 E-14$ & 0.29 & $0.47 \mathrm{E}-07$ & 25 \\
\hline & 2 & $0.15 E-07$ & $0.38 E-15$ & 0.64 & $0.19 E-07$ & 16 \\
\hline & 3 & $0.87 E-07$ & $0.28 E-14$ & 0.27 & $0.53 \mathrm{E}-07$ & 25 \\
\hline \multirow[t]{3}{*}{973} & 1 & $0.93 E-07$ & $0.26 E-14$ & 0.23 & $0.51 E-07$ & 16 \\
\hline & 2 & $0.95 E-08$ & $0.36 \mathrm{E}-16$ & 0.29 & $0.60 \mathrm{E}-08$ & 16 \\
\hline & 3 & $0.46 E-07$ & $0.15 \mathrm{E}-14$ & 0.43 & $0.39 E-07$ & 25 \\
\hline & 1 & $0.60 \mathrm{E}-07$ & $0.93 \mathrm{E}-14$ & 0.73 & $0.96 \mathrm{E}-07$ & 81 \\
\hline \multirow{2}{*}{988} & 1 & $0.12 \mathrm{E}-05$ & $0.29 E-10$ & 1.01 & $0.54 E-05$ & 16 \\
\hline & 2 & $0.11 E-06$ & $0.21 E-13$ & 0.65 & $0.14 \mathrm{E}-06$ & 64 \\
\hline \multirow[t]{3}{*}{1002} & 1 & $-0.12 \mathrm{E}-06$ & $0.48 E-13$ & 0.76 & $0.22 \mathrm{E}-06$ & 81 \\
\hline & 2 & $0.21 E-06$ & $0.37 E-13$ & 0.47 & $0.19 E-06$ & 25 \\
\hline & 3 & $0.61 \mathrm{E}-07$ & $0.11 E-14$ & 0.23 & $0.33 E-07$ & 81 \\
\hline 1003 & 1 & $-0.13 E-07$ & $0.22 \mathrm{E}-14$ & 0.95 & $0.47 E-07$ & 36 \\
\hline \multirow[t]{2}{*}{1008} & 1 & $0.12 \mathrm{E}-06$ & $0.16 \mathrm{E}-13$ & 0.55 & $0.13 \mathrm{E}-06$ & 16 \\
\hline & 2 & $0.24 \mathrm{E}-06$ & $0.81 E-12$ & 0.95 & $0.90 \mathrm{E}-06$ & 81 \\
\hline \multirow[t]{2}{*}{1009} & 1 & $0.36 \mathrm{E}-07$ & $0.46 \mathrm{E}-15$ & 0.27 & $0.21 E-07$ & 81 \\
\hline & 2 & $0.35 E-07$ & $0.40 E-14$ & 0.77 & $0.63 E-07$ & 81 \\
\hline \multirow[t]{2}{*}{1016} & 1 & $0.19 \mathrm{E}-06$ & $0.44 E-13$ & 0.57 & $0.21 \mathrm{E}-06$ & 25 \\
\hline & 2 & $0.41 E-07$ & $0.19 E-12$ & 1.01 & $0.43 E-06$ & 49 \\
\hline 1017 & 1 & $0.62 \mathrm{E}-07$ & $0.20 \mathrm{E}-14$ & 0.35 & $0.44 \mathrm{E}-07$ & 16 \\
\hline 1030 & 1 & $0.56 \mathrm{E}-07$ & $0.28 E-14$ & 0.47 & $0.53 E-07$ & 81 \\
\hline \multirow[t]{3}{*}{1031} & 1 & $0.73 E-07$ & $0.17 E-14$ & 0.25 & $0.42 \mathrm{E}-07$ & 36 \\
\hline & 2 & $-0.39 E-07$ & $0.15 E-14$ & 0.52 & $0.39 E-07$ & 16 \\
\hline & 3 & $-0.11 E-06$ & $0.43 E-14$ & 0.28 & $0.66 E-07$ & 16 \\
\hline 1051 & 1 & $0.35 E-06$ & $0.65 E-12$ & 0.85 & $0.81 E-06$ & 81 \\
\hline \multirow[t]{2}{*}{1052} & 1 & $0.27 E-06$ & $0.44 \mathrm{E}-12$ & 0.87 & $0.66 \mathrm{E}-06$ & 64 \\
\hline & 2 & $-0.11 E-06$ & $0.75 E-13$ & 0.90 & $0.27 E-06$ & 25 \\
\hline \multirow[t]{2}{*}{1059} & 1 & $0.17 E-06$ & $0.13 E-13$ & 0.33 & $0.12 E-06$ & 25 \\
\hline & 2 & $0.74 \mathrm{E}-09$ & $0.37 E-14$ & 1.04 & $0.61 E-07$ & 25 \\
\hline \multirow{5}{*}{1074} & 1 & $0.92 \mathrm{E}-0.8$ & $0.28 \mathrm{E}-15$ & 0.81 & $0.17 E-07$ & 16 \\
\hline & 1 & $0.87 E-07$ & $0.14 \mathrm{E}-11$ & 1.04 & $0.12 \mathrm{E}-05$ & 25 \\
\hline & 2 & $0.28 E-06$ & $0.13 E-11$ & 0.98 & $0.11 E-05$ & 25 \\
\hline & 3 & $0.16 \mathrm{E}-06$ & $0.36 \mathrm{E}-13$ & 0.61 & $0.19 \mathrm{E}-06$ & 25 \\
\hline & 4 & $0.51 \mathrm{E}-07$ & $0.12 E-14$ & 0.33 & $0.35 E-07$ & 16 \\
\hline
\end{tabular}


Table 24: Atlas SASS wind stress curl - 1-day averages

\begin{tabular}{rrrrrr} 
DAY & \multicolumn{1}{l}{ MEAN } & VARIANCE & WVAR & STND DEV & N \\
224 & $0.73 E-07$ & $0.38 \mathrm{E}-13$ & 0.88 & $0.19 \mathrm{E}-06$ & 182 \\
225 & $0.24 \mathrm{E}-07$ & $0.15 \mathrm{E}-13$ & 0.97 & $0.12 \mathrm{E}-06$ & 164 \\
226 & $0.42 \mathrm{E}-07$ & $0.15 \mathrm{E}-13$ & 0.90 & $0.12 \mathrm{E}-06$ & 235 \\
227 & $0.18 \mathrm{E}-06$ & $0.16 \mathrm{E}-12$ & 0.83 & $0.40 \mathrm{E}-06$ & 251 \\
228 & $0.19 \mathrm{E}-06$ & $0.19 \mathrm{E}-12$ & 0.85 & $0.44 \mathrm{E}-06$ & 225 \\
229 & $0.64 \mathrm{E}-08$ & $0.28 \mathrm{E}-13$ & 1.00 & $0.17 \mathrm{E}-06$ & 375 \\
230 & $0.23 \mathrm{E}-07$ & $0.19 \mathrm{E}-13$ & 0.98 & $0.14 \mathrm{E}-06$ & 435 \\
231 & $-0.65 \mathrm{E}-07$ & $0.83 \mathrm{E}-14$ & 0.67 & $0.91 \mathrm{E}-07$ & 66 \\
232 & $-0.39 \mathrm{E}-07$ & $0.12 \mathrm{E}-12$ & 0.99 & $0.34 \mathrm{E}-06$ & 234 \\
233 & $-0.12 \mathrm{E}-06$ & $0.11 \mathrm{E}-12$ & 0.88 & $0.34 \mathrm{E}-06$ & 316 \\
234 & $-0.30 \mathrm{E}-07$ & $0.54 \mathrm{E}-13$ & 0.99 & $0.23 \mathrm{E}-06$ & 307 \\
235 & $0.23 \mathrm{E}-07$ & $0.68 \mathrm{E}-13$ & 1.00 & $0.26 \mathrm{E}-06$ & 370 \\
236 & $-0.26 \mathrm{E}-07$ & $0.71 \mathrm{E}-13$ & 0.99 & $0.27 \mathrm{E}-06$ & 421 \\
237 & $0.23 \mathrm{E}-07$ & $0.42 \mathrm{E}-14$ & 0.89 & $0.65 \mathrm{E}-07$ & 122 \\
238 & $-0.72 \mathrm{E}-07$ & $0.13 \mathrm{E}-12$ & 0.96 & $0.35 \mathrm{E}-06$ & 317 \\
239 & $-0.31 \mathrm{E}-07$ & $0.57 \mathrm{E}-13$ & 0.99 & $0.24 \mathrm{E}-06$ & 212 \\
240 & $-0.38 \mathrm{E}-07$ & $0.33 \mathrm{E}-13$ & 0.96 & $0.18 \mathrm{E}-06$ & 244 \\
241 & $-0.95 \mathrm{E}-07$ & $0.58 \mathrm{E}-13$ & 0.87 & $0.24 \mathrm{E}-06$ & 273 \\
242 & $-0.12 \mathrm{E}-06$ & $0.67 \mathrm{E}-13$ & 0.84 & $0.26 \mathrm{E}-06$ & 136 \\
243 & $0.42 \mathrm{E}-08$ & $0.86 \mathrm{E}-14$ & 1.02 & $0.93 \mathrm{E}-07$ & 50 \\
244 & $0.49 \mathrm{E}-07$ & $0.58 \mathrm{E}-13$ & 0.97 & $0.24 \mathrm{E}-06$ & 164 \\
245 & $0.36 \mathrm{E}-07$ & $0.15 \mathrm{E}-13$ & 0.93 & $0.12 \mathrm{E}-06$ & 123 \\
246 & $0.11 \mathrm{E}-06$ & $0.29 \mathrm{E}-12$ & 0.96 & $0.54 \mathrm{E}-06$ & 218 \\
247 & $0.41 \mathrm{E}-07$ & $0.18 \mathrm{E}-12$ & 0.99 & $0.42 \mathrm{E}-06$ & 384 \\
248 & $0.42 \mathrm{E}-08$ & $0.69 \mathrm{E}-12$ & 1.00 & $0.83 \mathrm{E}-06$ & 572 \\
249 & $0.78 \mathrm{E}-07$ & $0.72 \mathrm{E}-13$ & 0.93 & $0.27 \mathrm{E}-06$ & 239 \\
250 & $0.52 \mathrm{E}-07$ & $0.20 \mathrm{E}-13$ & 0.89 & $0.14 \mathrm{E}-06$ & 149 \\
251 & $0.11 \mathrm{E}-06$ & $0.50 \mathrm{E}-12$ & 0.98 & $0.70 \mathrm{E}-06$ & 236 \\
252 & $0.24 \mathrm{E}-06$ & $0.30 \mathrm{E}-12$ & 0.85 & $0.55 \mathrm{E}-06$ & 157
\end{tabular}


Table 25: FNOC wind stress curl - 1-day averages

\begin{tabular}{lrlllr} 
DAY & \multicolumn{1}{l}{ MEAN } & VARIANCE & WVAR & STND DEV & N \\
224 & $0.38 E-07$ & $0.63 E-14$ & 0.82 & $0.80 E-07$ & 182 \\
225 & $0.55 E-07$ & $0.65 E-14$ & 0.68 & $0.81 E-07$ & 164 \\
226 & $0.45 E-07$ & $0.85 E-14$ & 0.81 & $0.92 E-07$ & 235 \\
227 & $0.12 E-06$ & $0.50 E-13$ & 0.79 & $0.22 E-06$ & 251 \\
228 & $0.12 E-06$ & $0.54 E-13$ & 0.79 & $0.23 E-06$ & 225 \\
229 & $-0.19 E-07$ & $0.29 E-13$ & 0.99 & $0.17 E-06$ & 375 \\
230 & $0.13 E-07$ & $0.21 E-13$ & 0.99 & $0.15 E-06$ & 435 \\
231 & $0.11 E-07$ & $0.52 E-14$ & 0.99 & $0.72 E-07$ & 66 \\
232 & $0.16 E-08$ & $0.28 E-13$ & 1.00 & $0.17 E-06$ & 234 \\
233 & $-0.21 E-07$ & $0.42 E-13$ & 0.99 & $0.20 E-06$ & 316 \\
234 & $-0.14 E-07$ & $0.23 E-13$ & 1.00 & $0.15 E-06$ & 307 \\
235 & $0.10 E-07$ & $0.29 E-13$ & 1.00 & $0.17 E-06$ & 370 \\
236 & $-0.13 E-07$ & $0.46 E-13$ & 1.00 & $0.21 E-06$ & 421 \\
237 & $0.22 E-07$ & $0.14 E-14$ & 0.76 & $0.38 E-07$ & 122 \\
238 & $-0.40 E-07$ & $0.36 E-13$ & 0.96 & $0.19 E-06$ & 317 \\
239 & $-0.39 E-07$ & $0.40 E-13$ & 0.97 & $0.20 E-06$ & 212 \\
240 & $-0.25 E-07$ & $0.20 E-13$ & 0.97 & $0.14 E-06$ & 244 \\
241 & $-0.34 E-07$ & $0.21 E-13$ & 0.95 & $0.14 E-06$ & 273 \\
242 & $-0.24 E-07$ & $0.90 E-14$ & 0.95 & $0.95 E-07$ & 136 \\
243 & $0.14 E-07$ & $0.37 E-14$ & 0.97 & $0.61 E-07$ & 50 \\
244 & $-0.12 E-06$ & $0.44 E-11$ & 1.00 & $0.21 E-05$ & 164 \\
245 & $0.27 E-07$ & $0.52 E-14$ & 0.88 & $0.72 E-07$ & 123 \\
246 & $0.16 E-06$ & $0.21 E-11$ & 0.99 & $0.15 E-05$ & 218 \\
247 & $0.80 E-07$ & $0.12 E-11$ & 1.00 & $0.11 E-05$ & 384 \\
248 & $0.59 E-07$ & $0.15 E-12$ & 0.98 & $0.39 E-06$ & 572 \\
249 & $0.52 E-07$ & $0.48 E-13$ & 0.95 & $0.22 E-06$ & 239 \\
250 & $0.33 E-07$ & $0.59 E-14$ & 0.85 & $0.77 E-07$ & 149 \\
251 & $0.20 E-06$ & $0.38 E-12$ & 0.91 & $0.61 E-06$ & 236 \\
252 & $0.12 E-06$ & $0.42 E-12$ & 0.98 & $0.65 E-06$ & 157
\end{tabular}




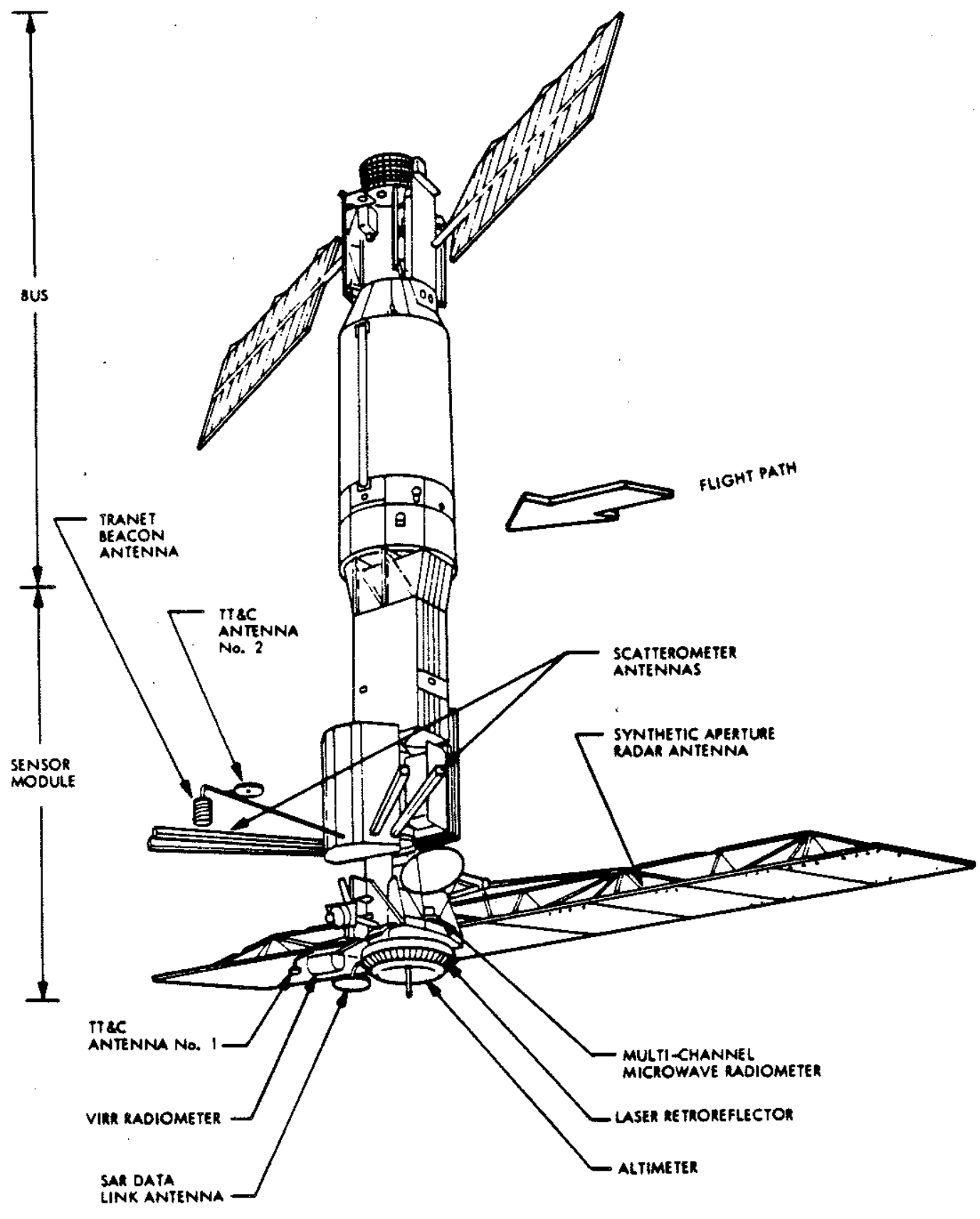

Figure 1: Seasat Configuration (from Boggs, 1982) 


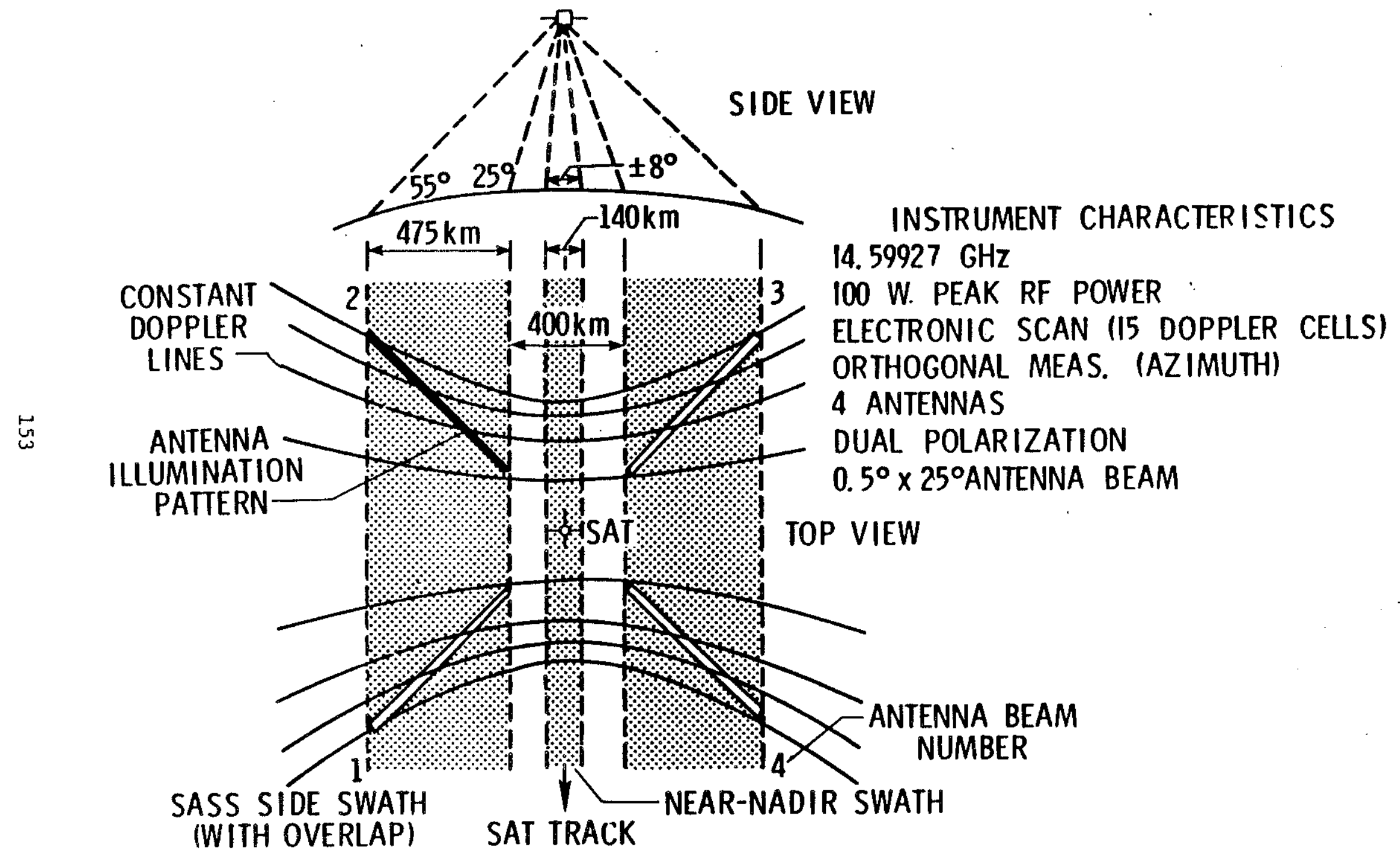

Figure 2: SEASAT scatterometer illumination pattern and instrument characteristics (from Boggs, 1982) 


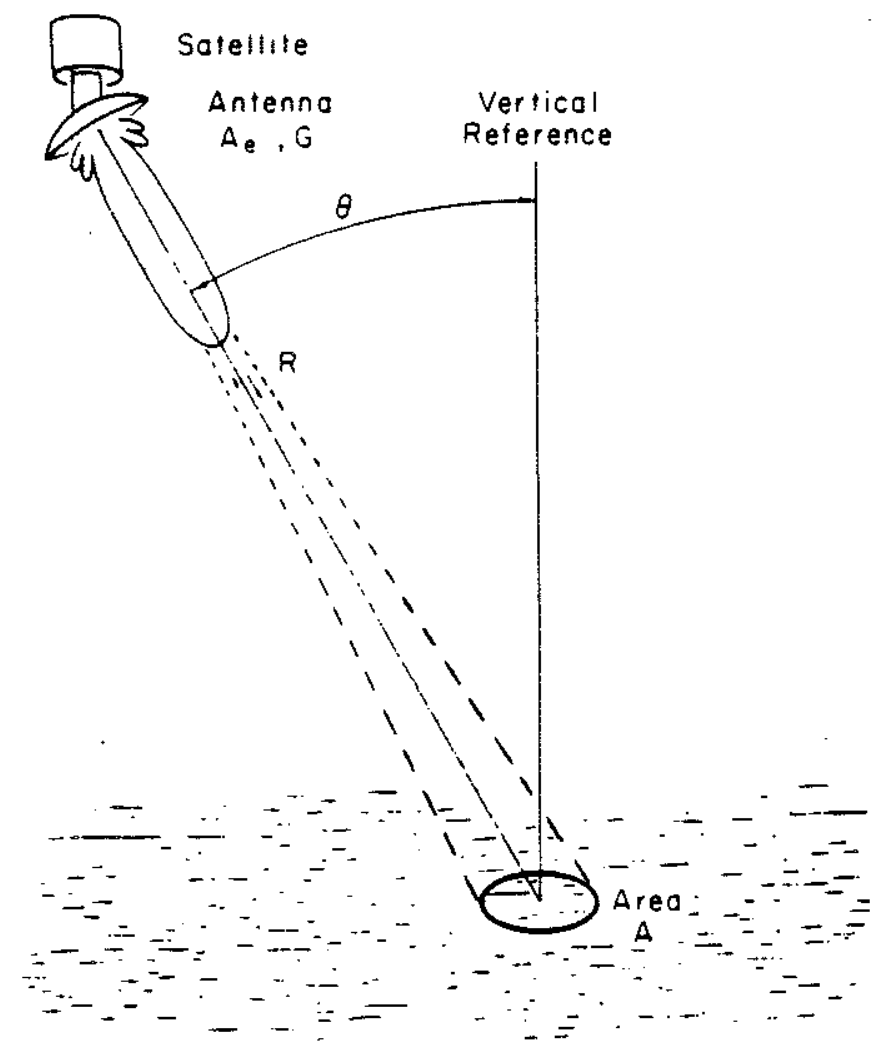

Figure 3: Radar geometry for scatter from a sea surface. (from Stewart, 1985)

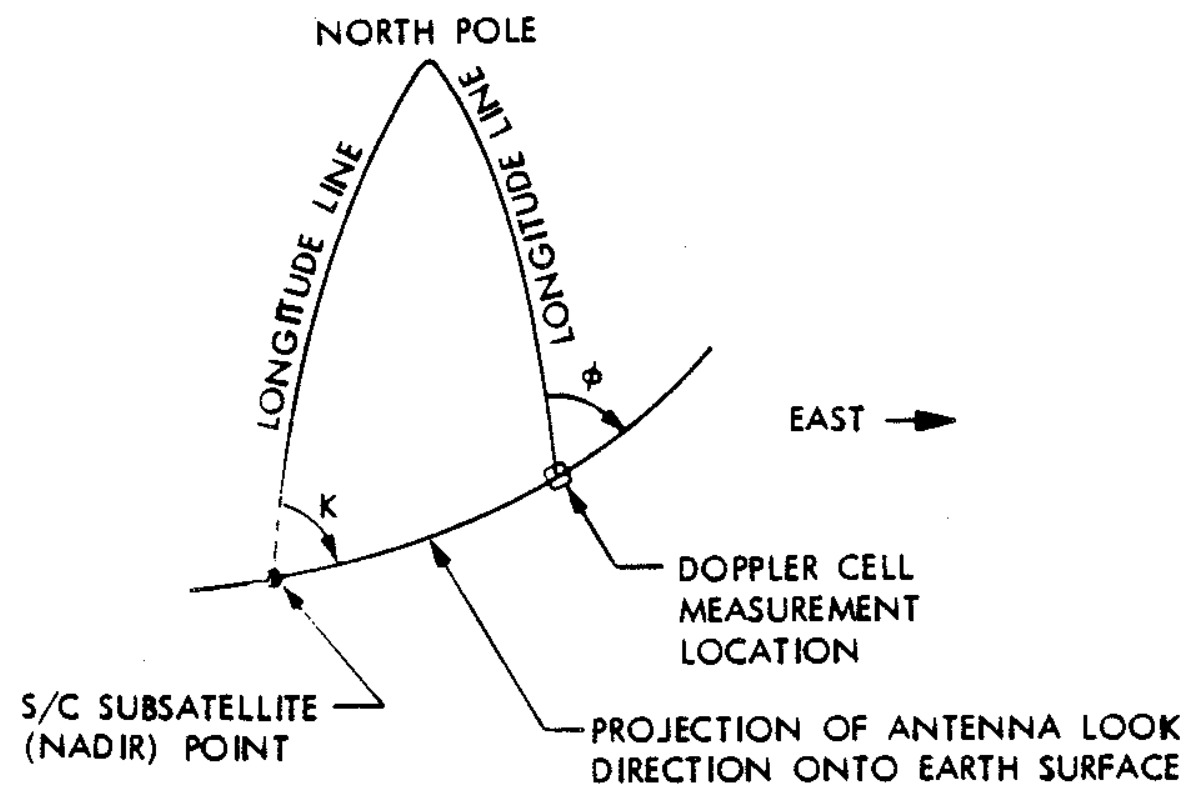

Figure 4: Projected antenna look direction and local vertical incidence angle (from Boggs, 1982) 

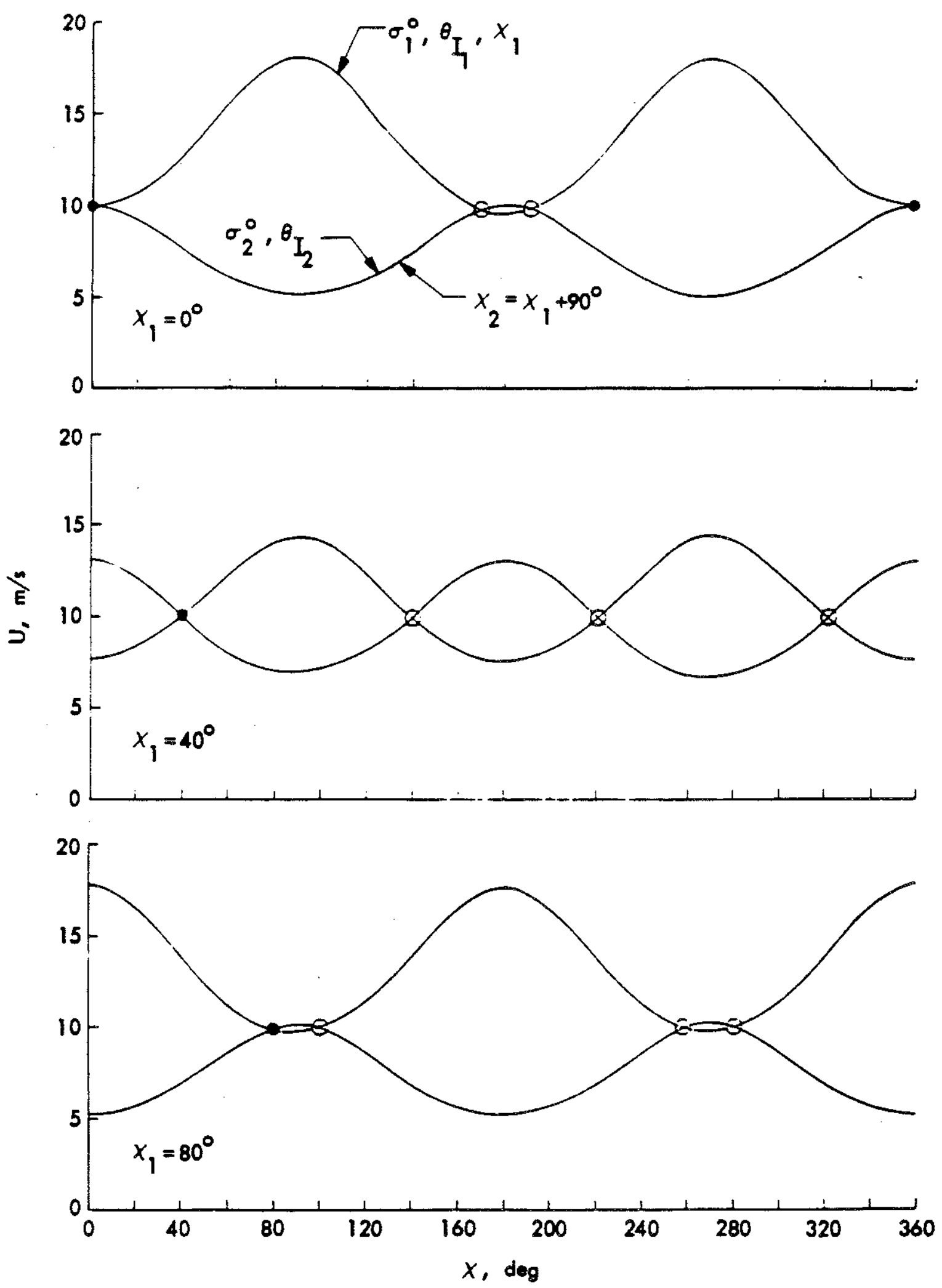

Figure 5: Examples of noise-free solutions for beam patterns $90^{\circ}$ apart, vertical polarization, and a wind speed of $10 \mathrm{~m} / \mathrm{s}$ for wind directions of $X=0^{\circ}, 40^{\circ}$, and $80^{\circ}$ (from Boggs, 1982) 


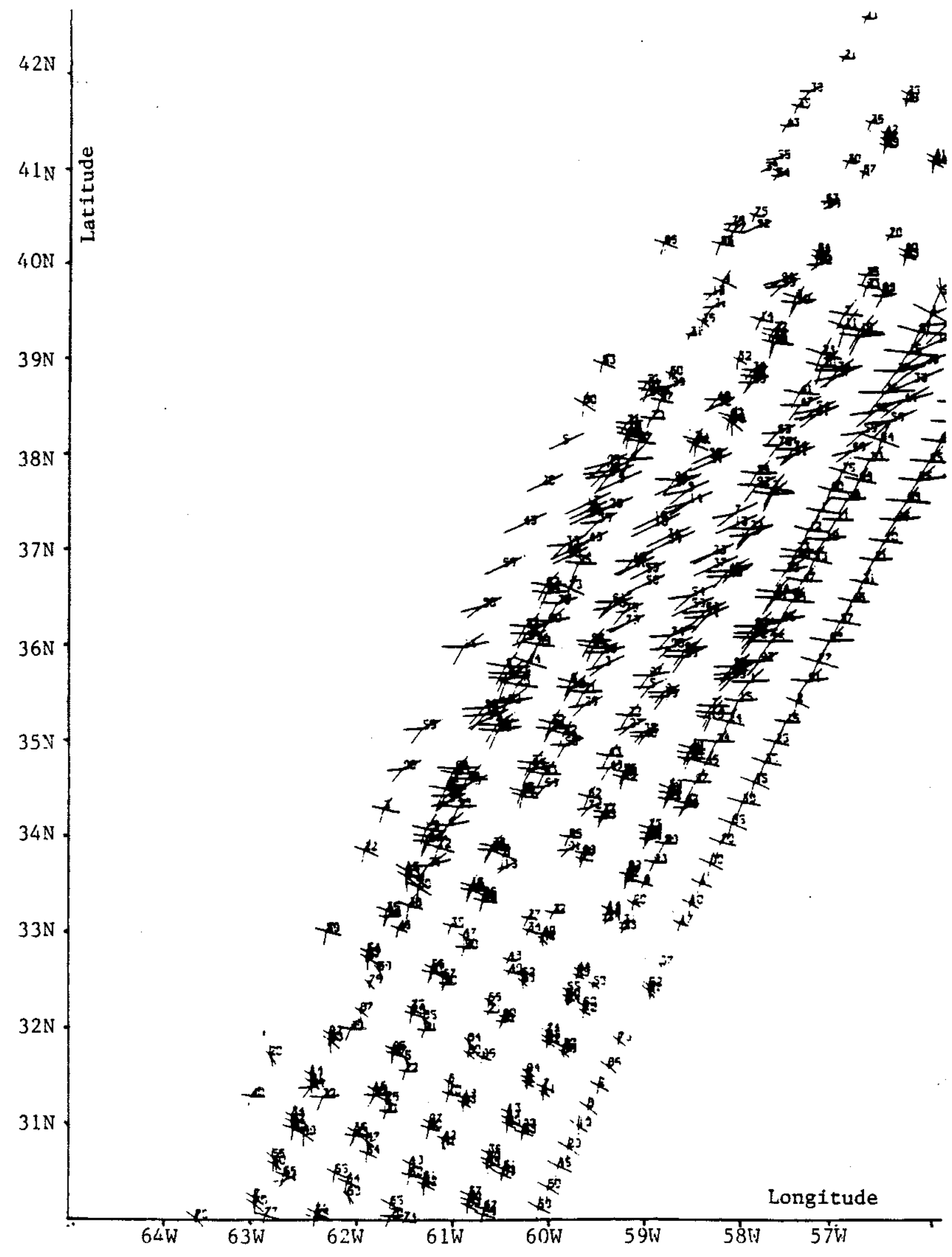

Figure 6: Aliased GDR SASS wind vectors - Rev 184 


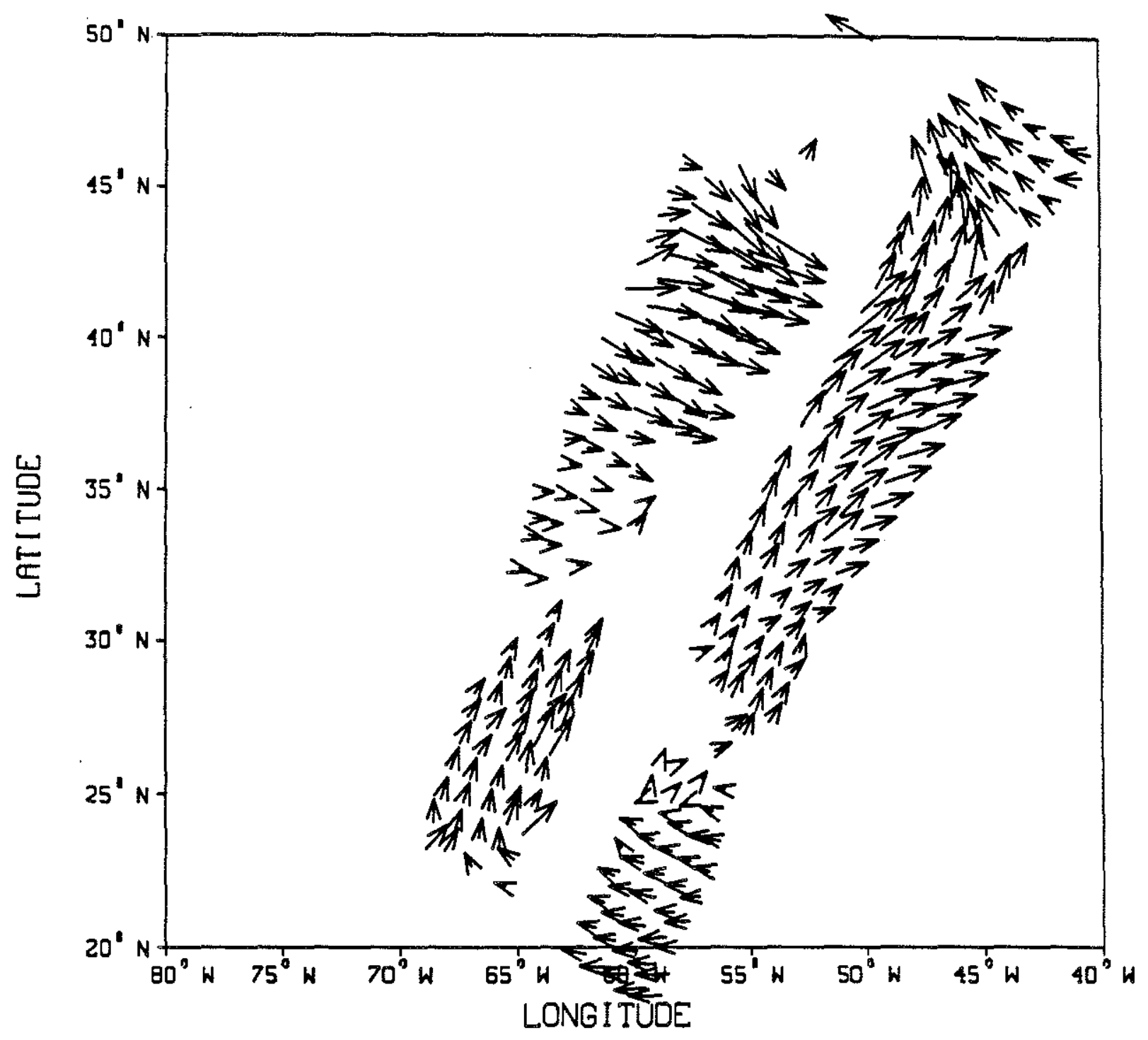

Figure 7: Ungridded Atlas SASS winds - Rev 830 


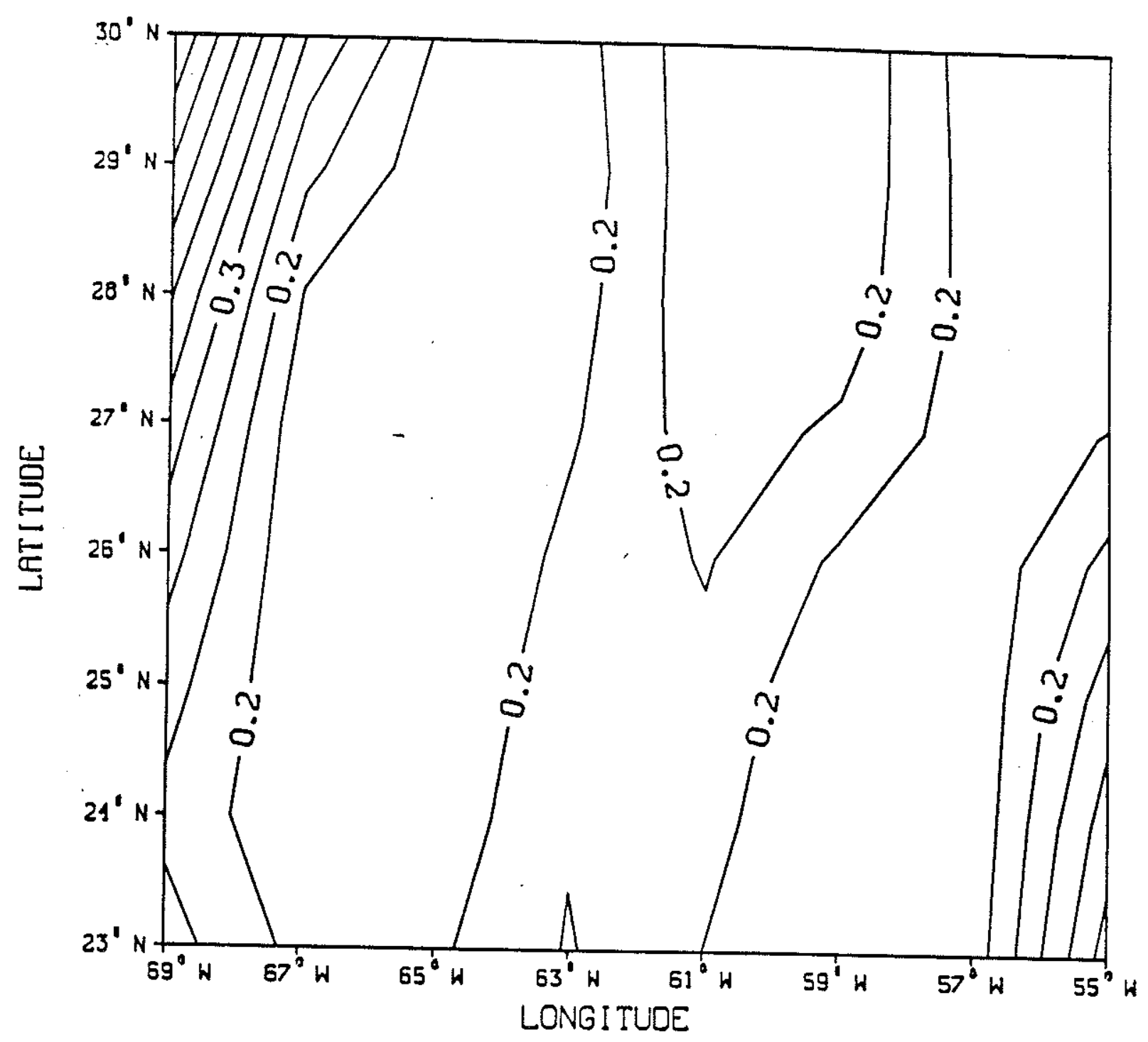

Figure 8: Objective error map - normalized standard deviation
Rev 8303 


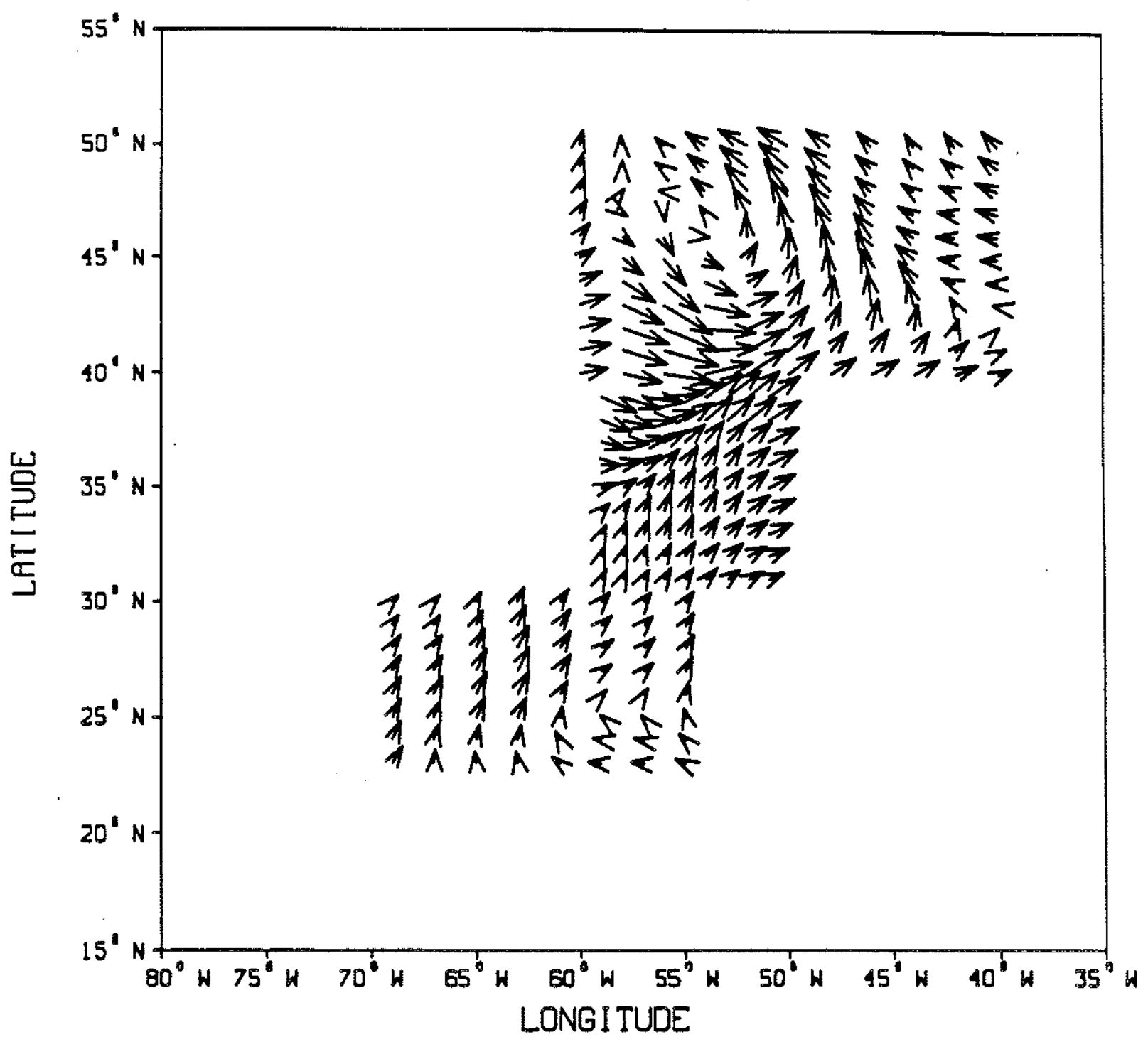

Figure 9: Mapped Atlas winds - Rev 830 

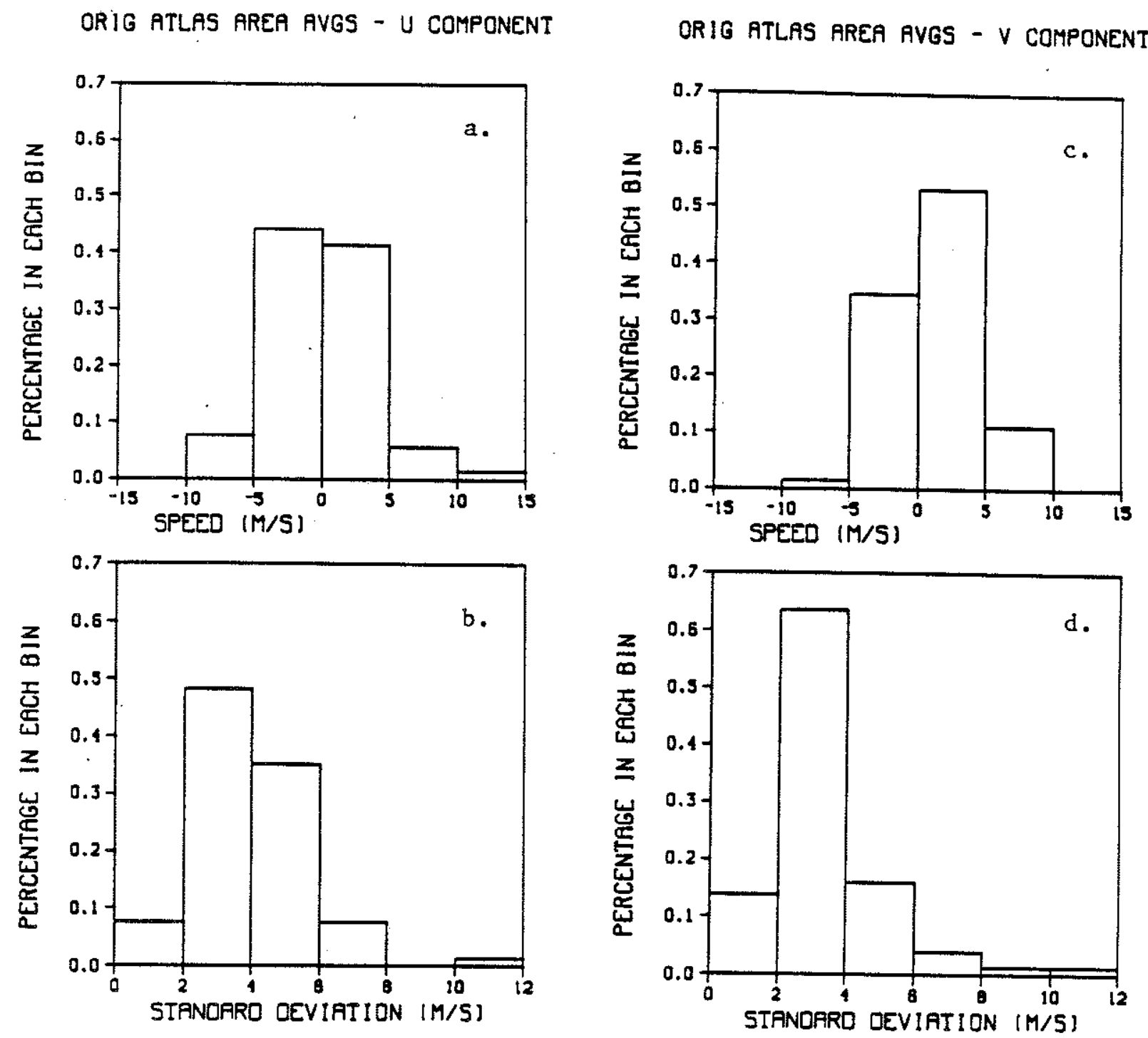

Figure 10: Distribution of ungridded Atlas wind components - averaged over individual boxes. a. U velocity, b. U stnd dev., c. V velocity, d. V stnd dev.

$\mathrm{N}=145$ pts 

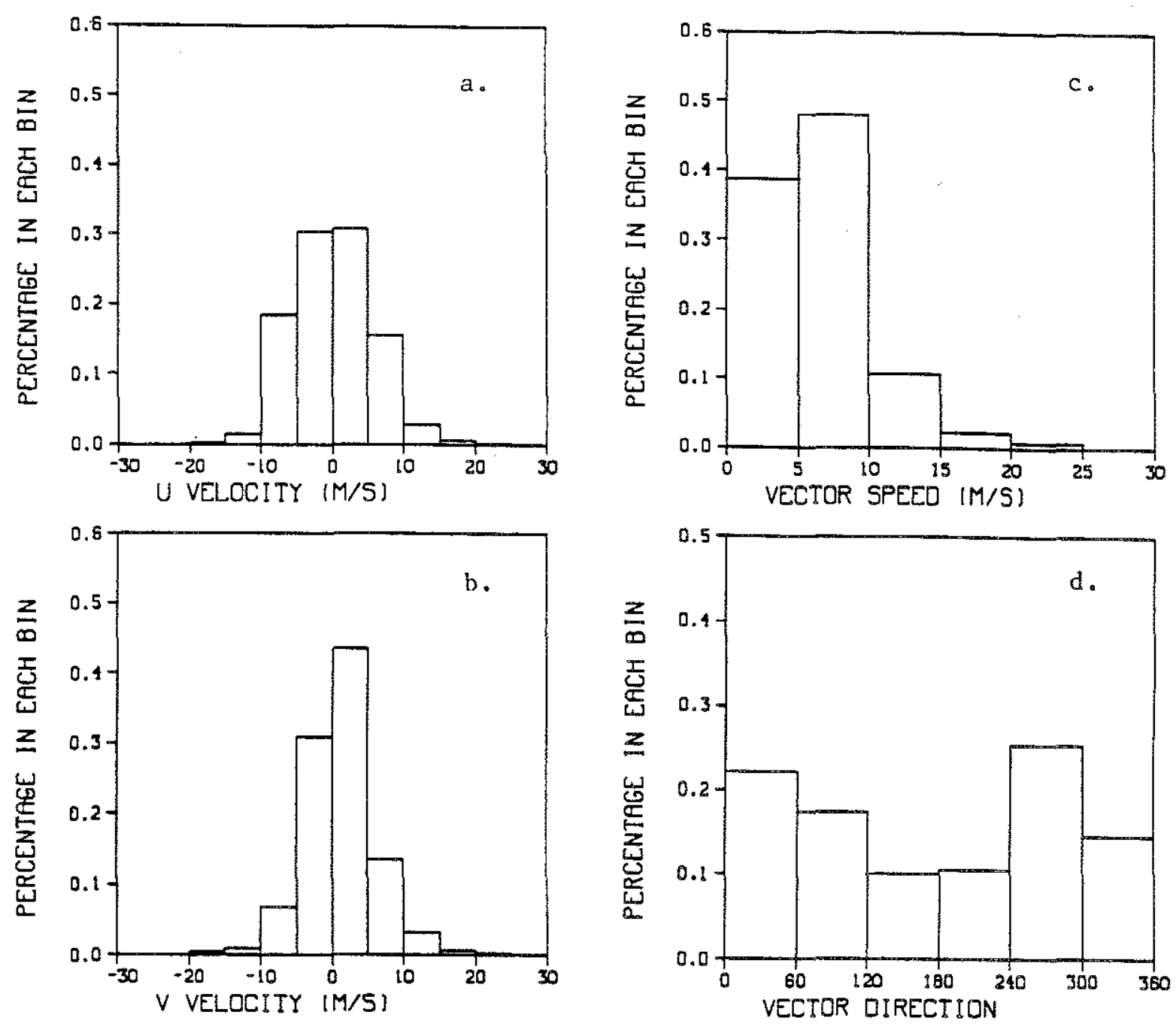

Figure 11: Distribution of all individual mapped Atlas wind vectors a. U velocity, b. $V$ velocity, $c$. vector speed, d. vector direction $\mathrm{N}=8214$ pts 
MAP ATLAS AREA AVGS - U COMPONENT

MAP ATLAS AREA AVGS - $V$ COMPONENT
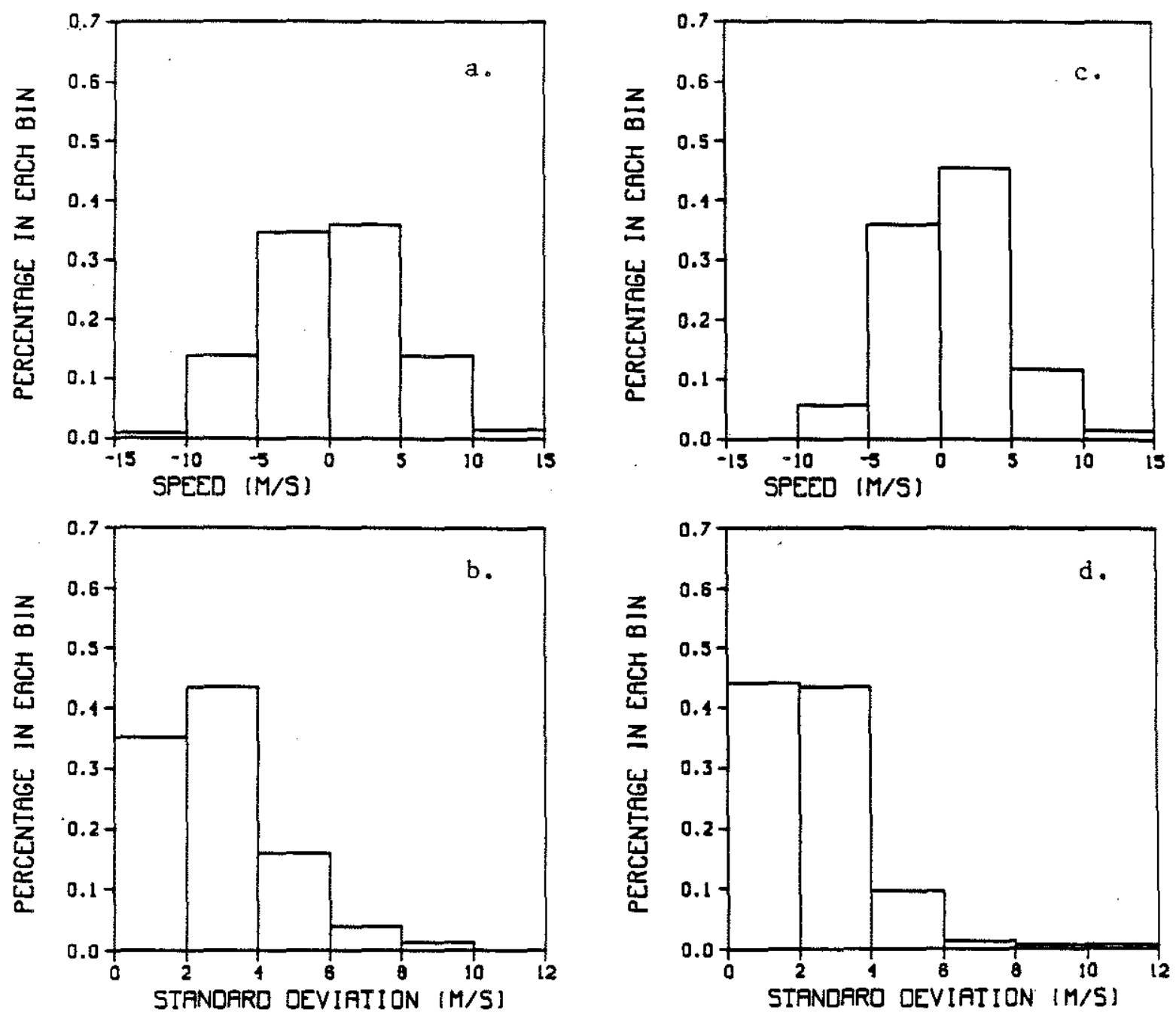

Figure 12: Distribution of mapped Atlas wind components - averaged over individual boxes. a. U velocity, b. U stnd dev., c. V velocity, d. $V$ stnd dev. $\mathrm{N}=145 \mathrm{pts}$ 
FNOC INDIVIDUAL VECTORS - N-8214

FNOC INDIVIOUAL VECTORS - N-8214
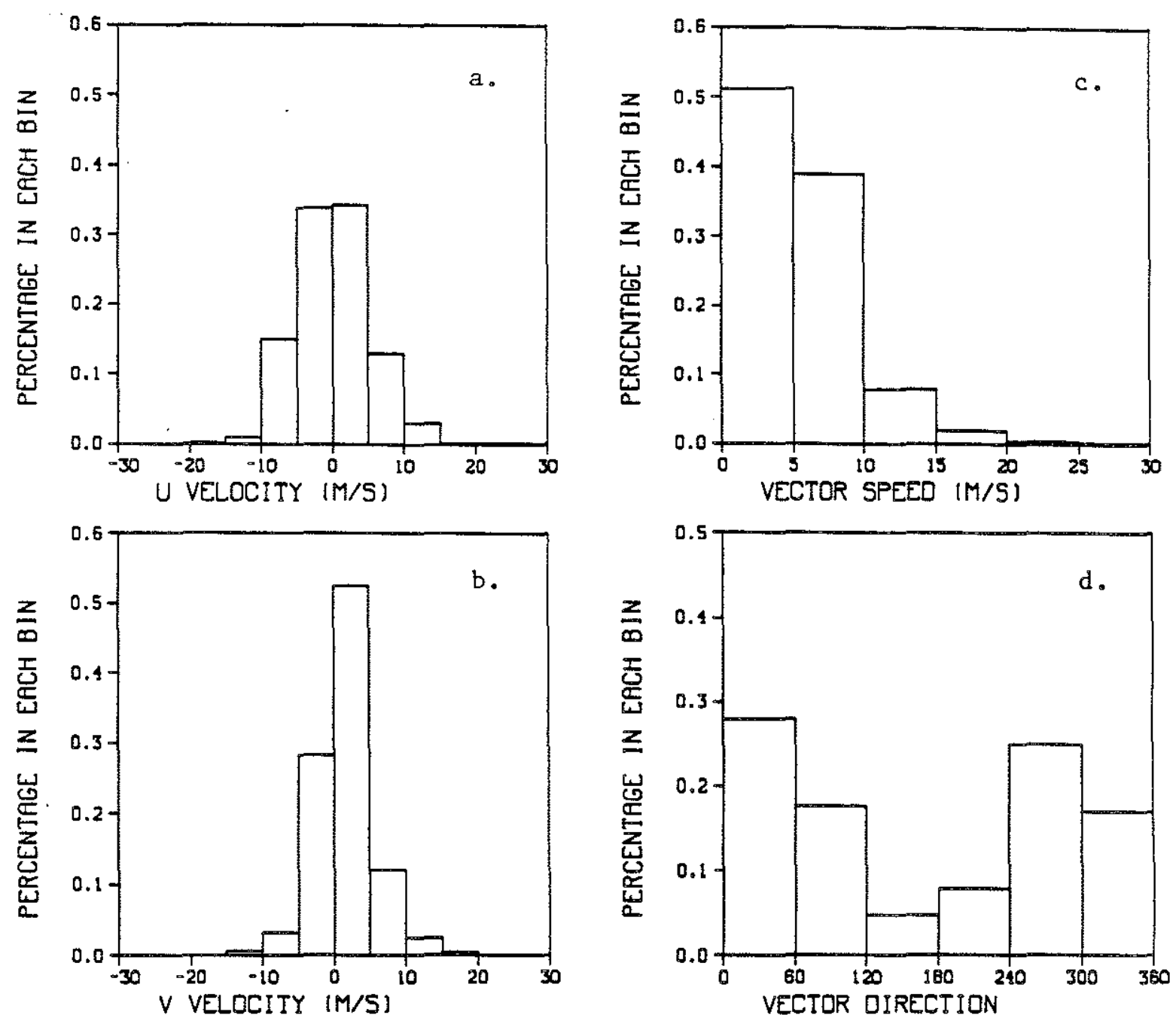

Figure 13: Distribution of all individual FNOC wind vectors

a. U velocity, b. V velocity, c, vector speed,

d. vector direction

$\mathrm{N}=8214$ pts 
FNOC AREA RVGS - U COMPONENT
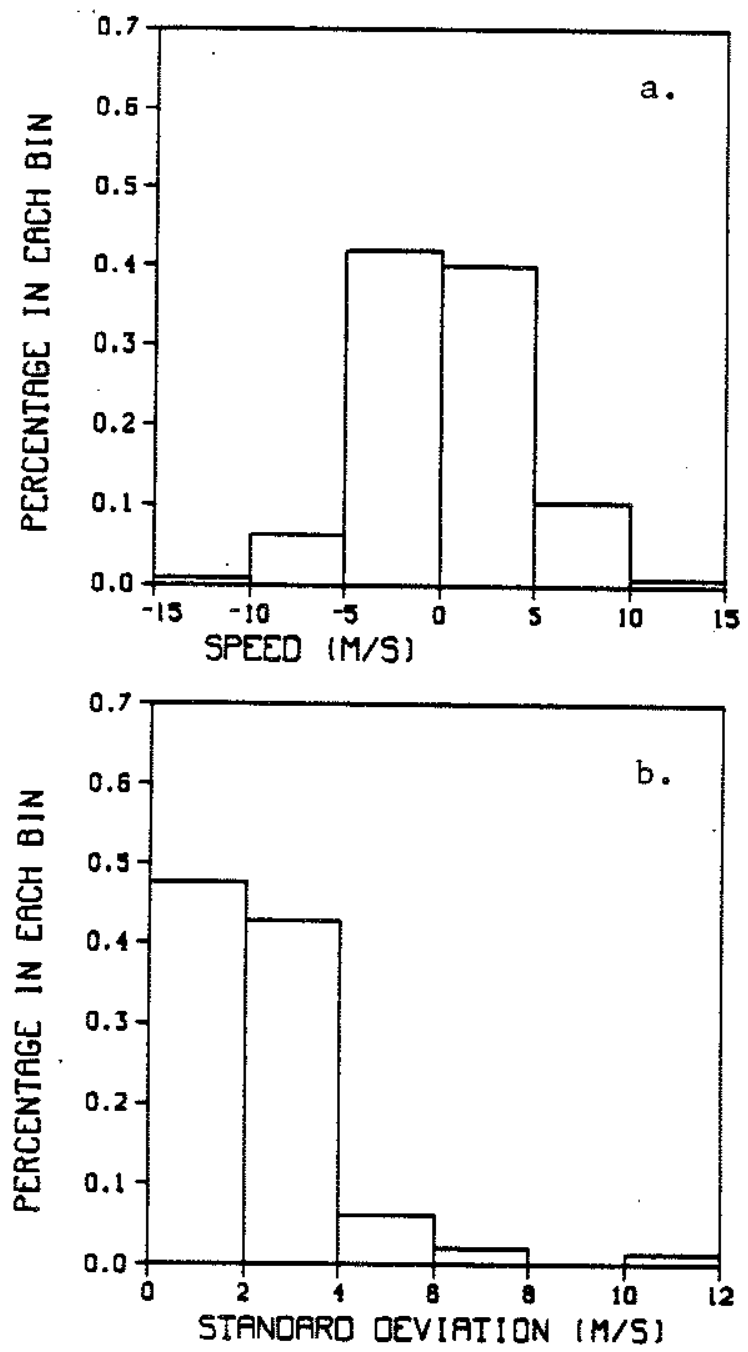

FNOC AREA RVGS - $V$ COMPONENT
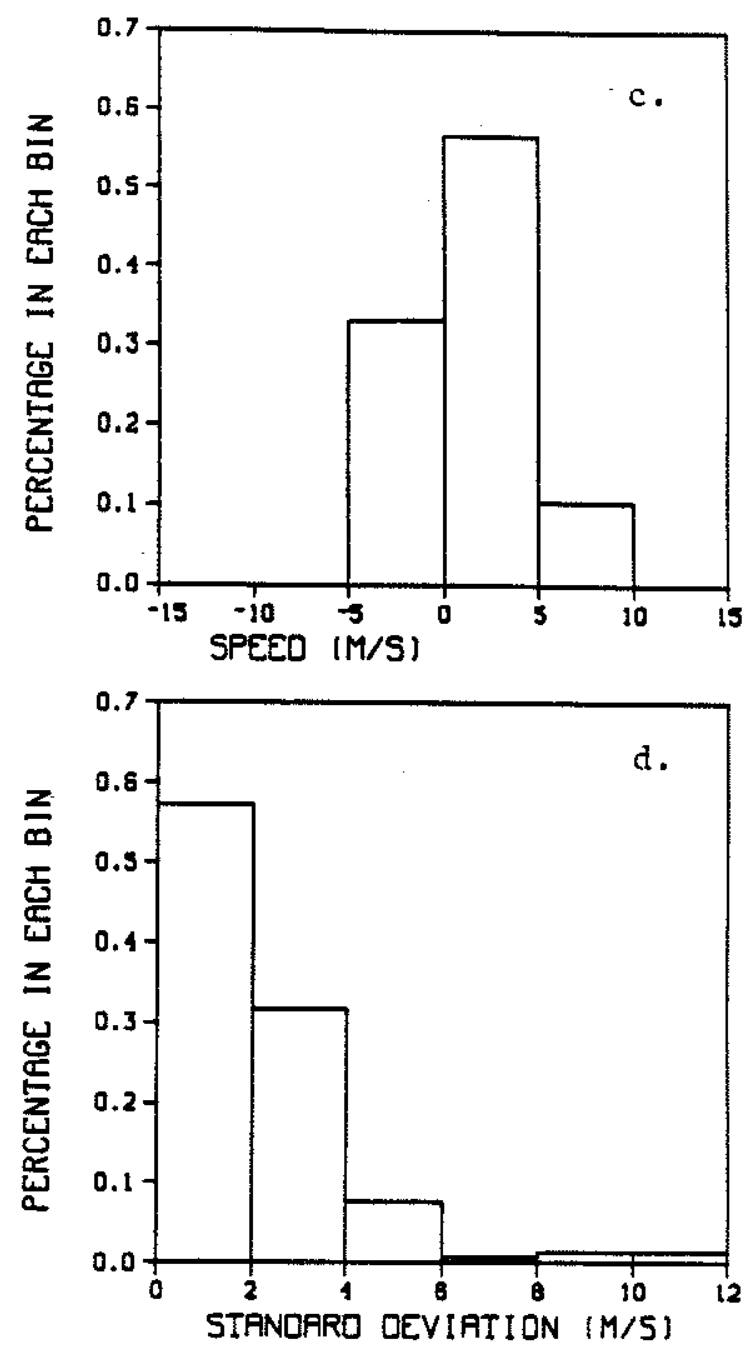

Figure 14: Distribution of FNOC wind components - averaged over individual boxes, a. U velocity, b. U stnd dev., c. V velocity, d. $V$ stnd. dev. $\mathrm{N}=145 \mathrm{pts}$ 

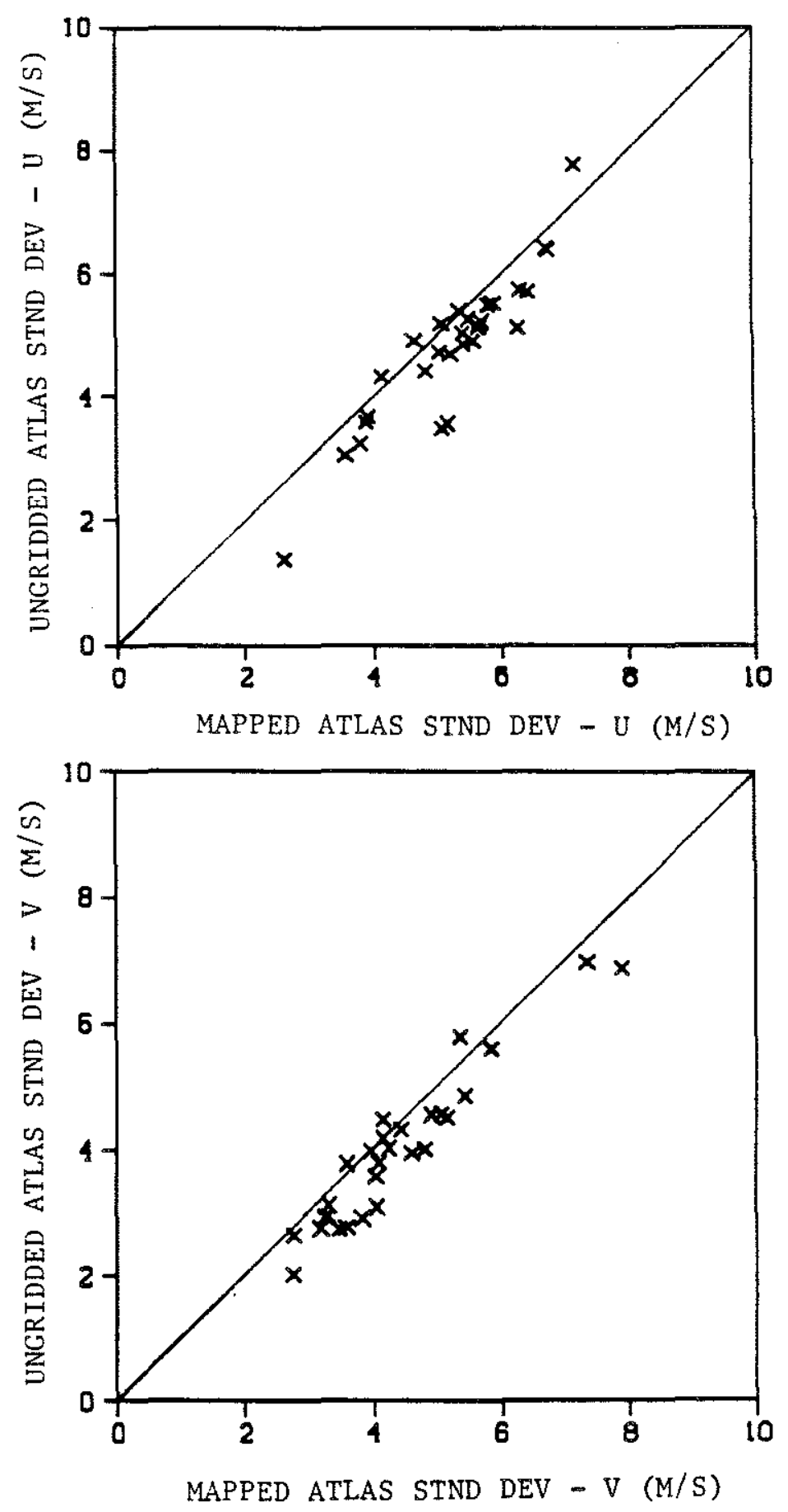

Figure 15: Mapped vs, ungridded Atlas wind component standard deviations - from 1-day averages 

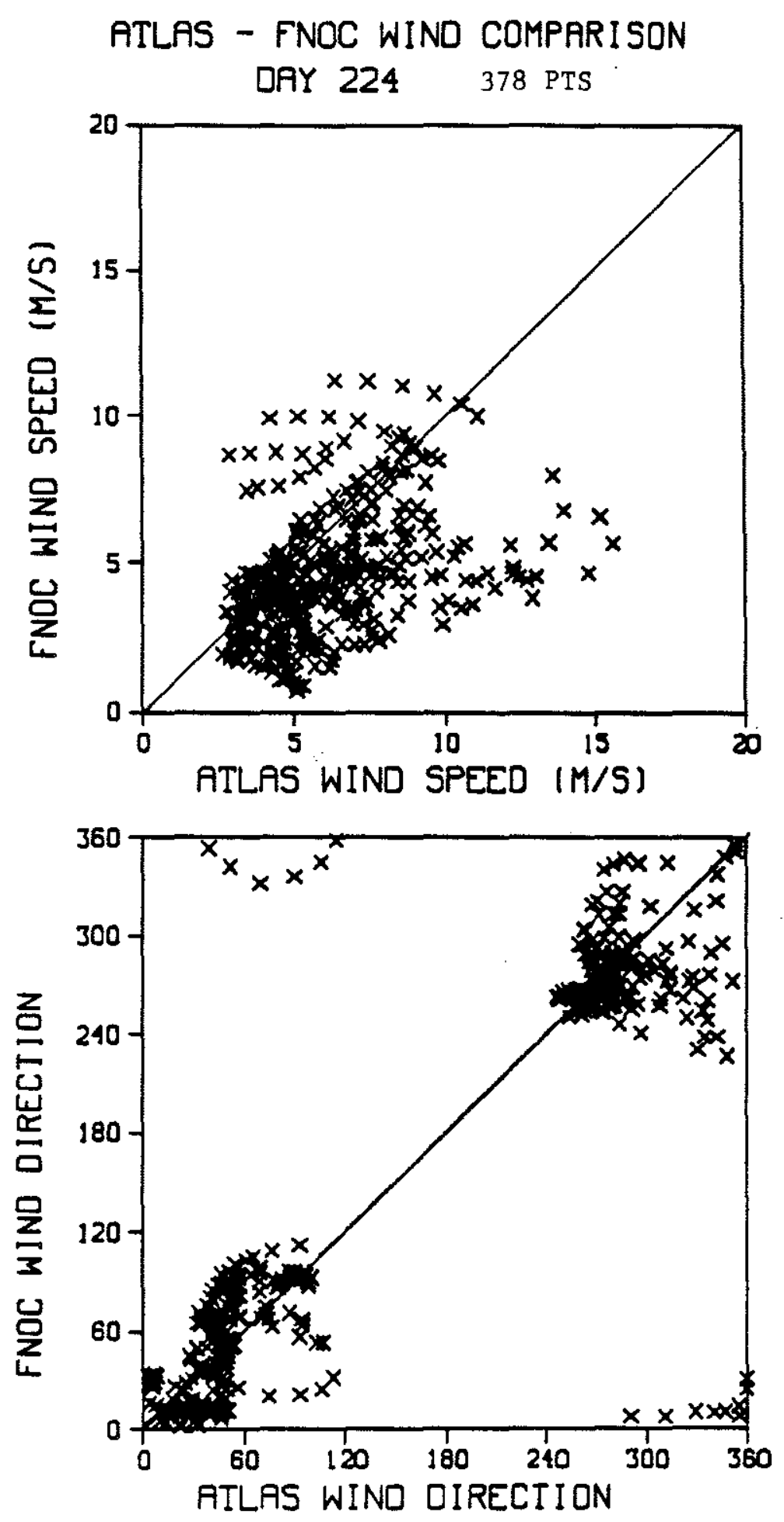

Figure 16: Mapped Atlas vs FNOC wind speed and direction - 1-day period 

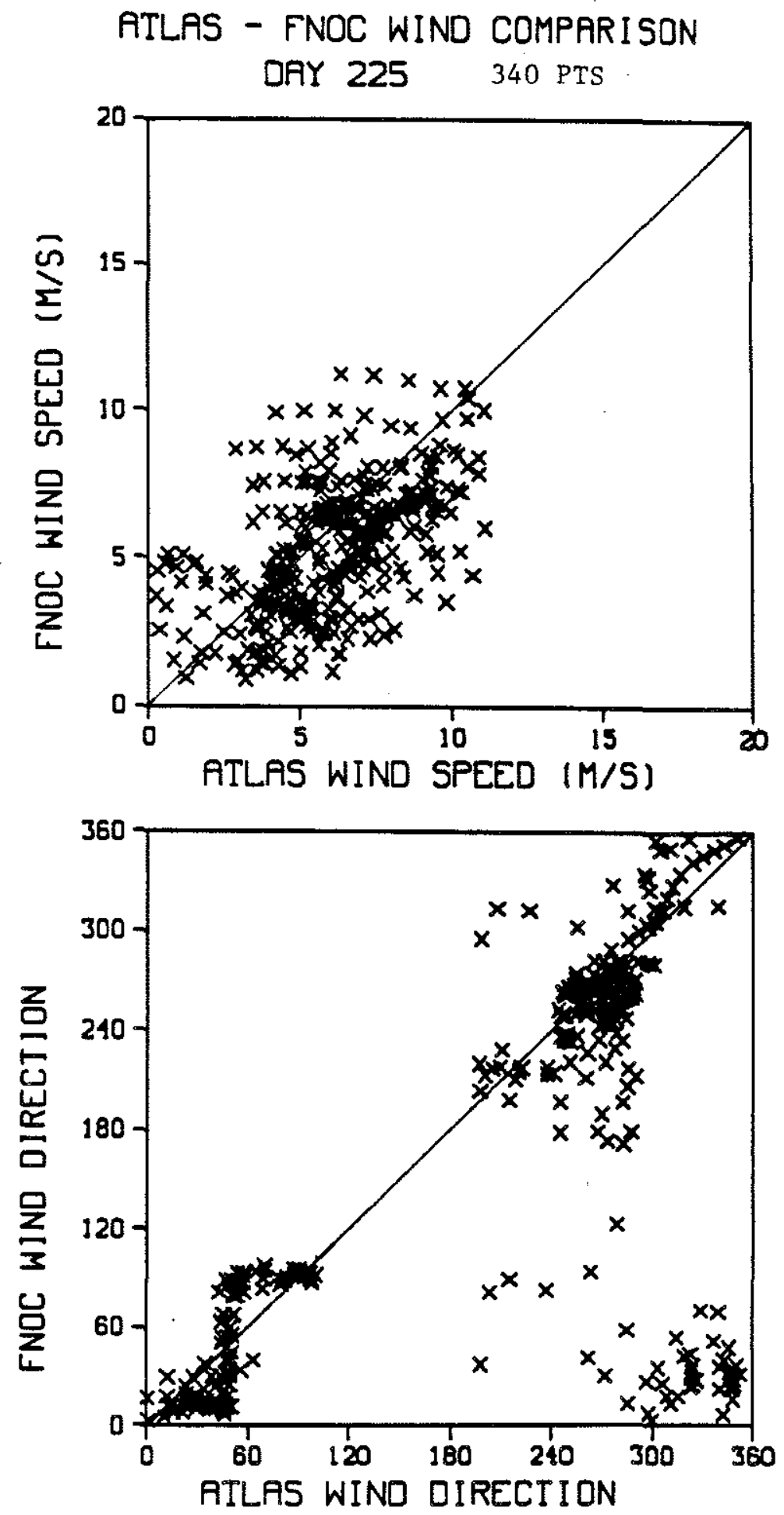

Figure 17: Mapped Atlas vs FNOC wind speed and direction - 1-day period 

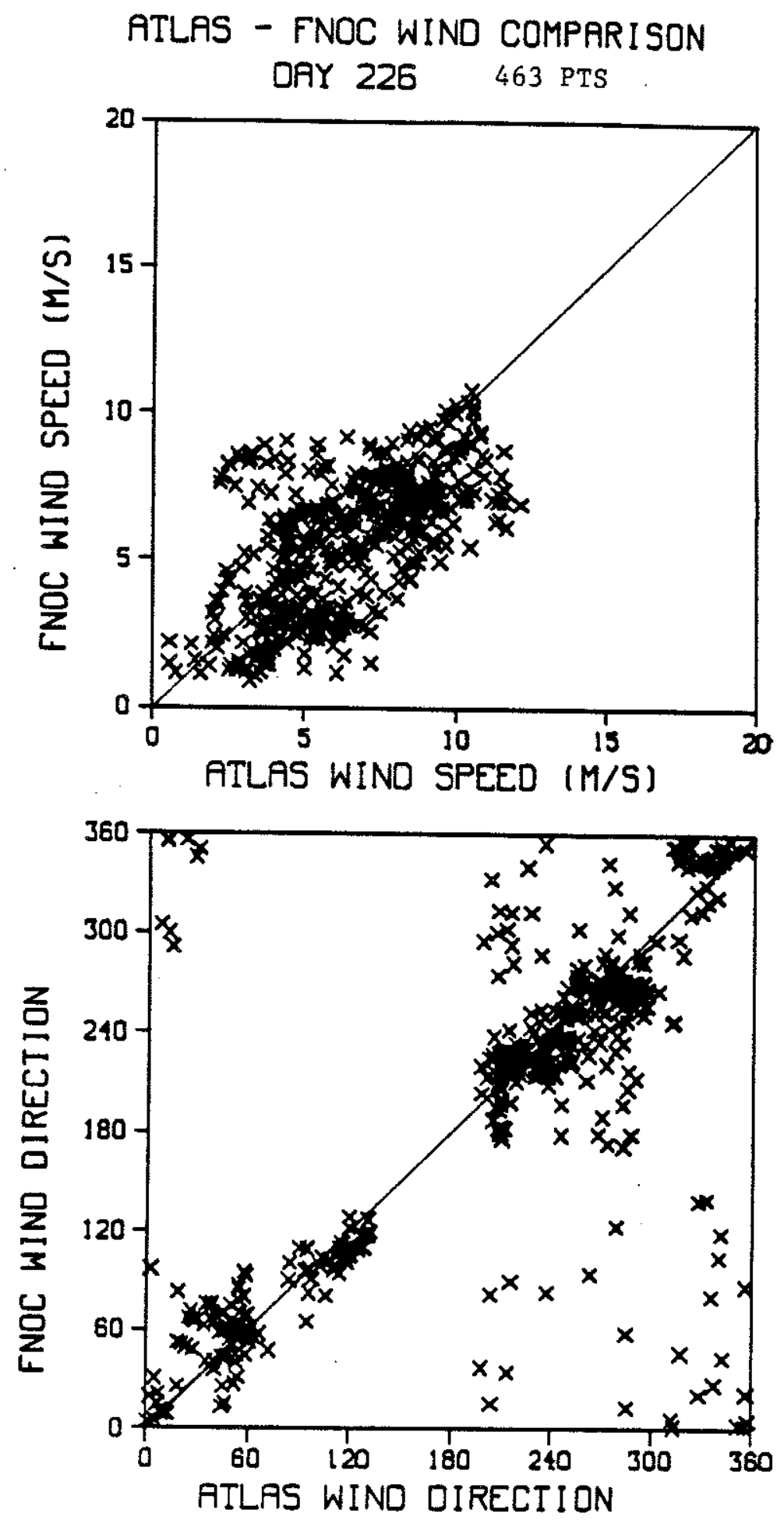

Figure 18: Mapped Atlas vs FNOC wind speed and direction - 1-day period 

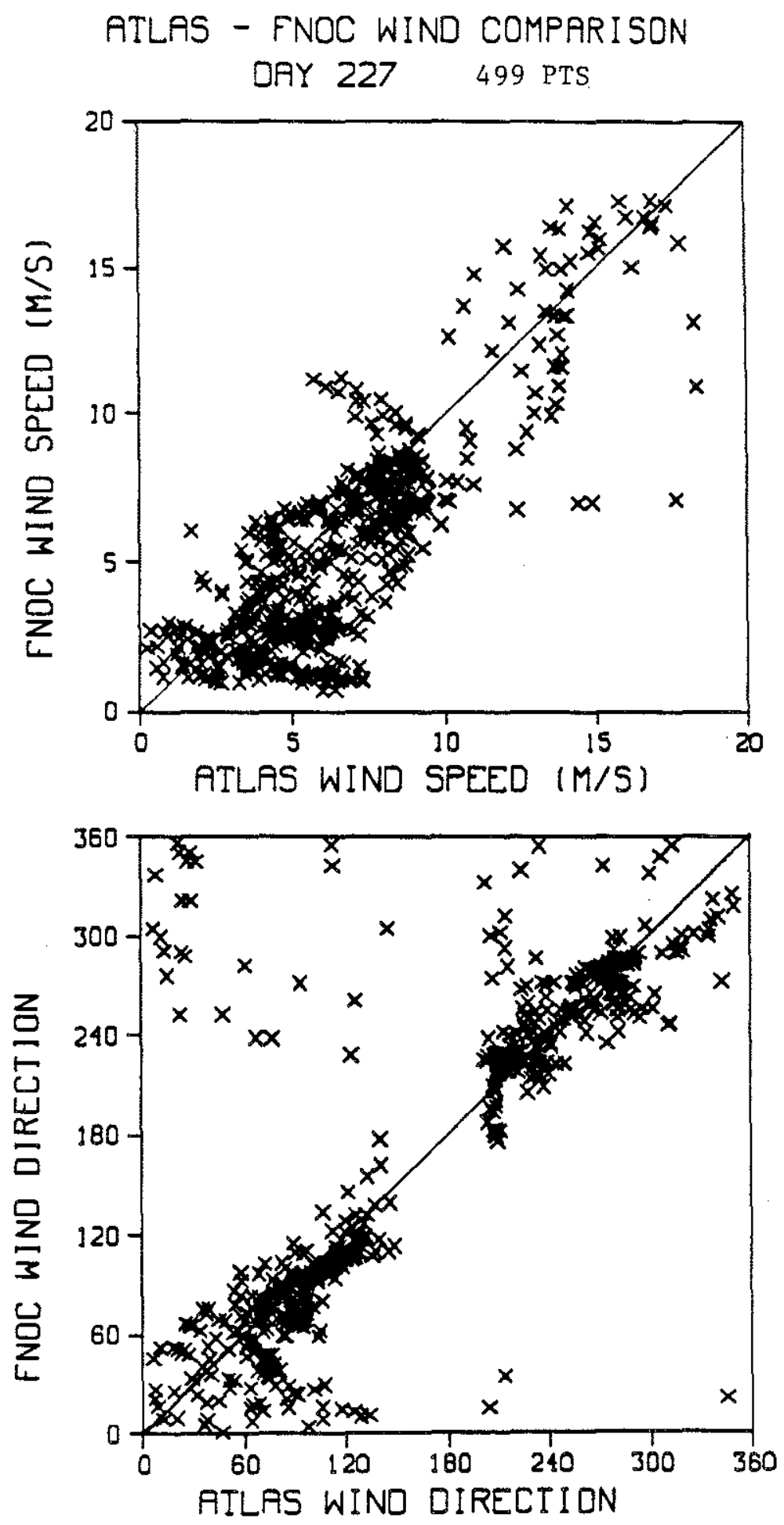

Figure 19: Mapped Atlas vs FNOC wind speed and direction - 1-day period 

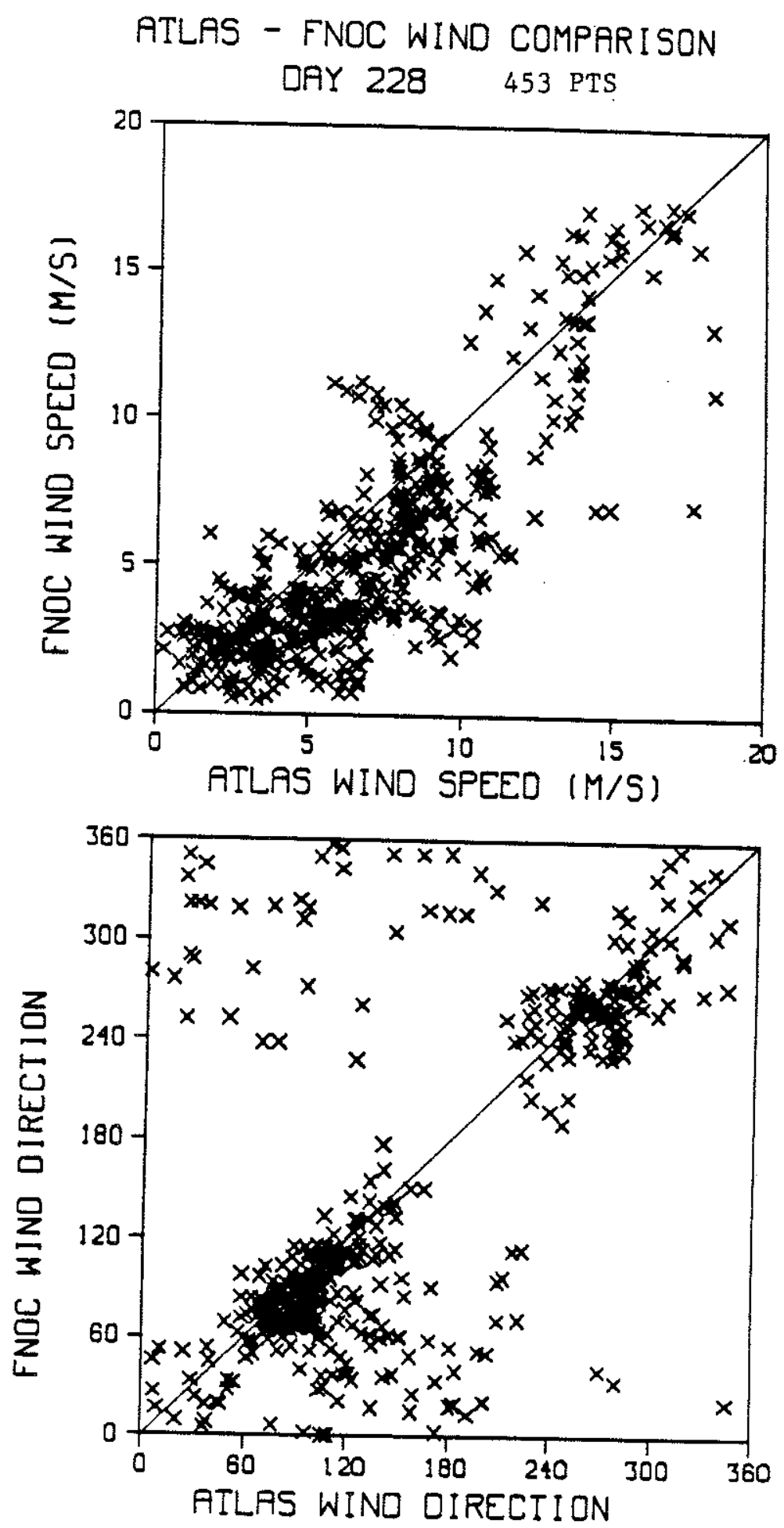

Figure 20: Mapped Atlas vs FNOC wind speed and direction - 1-day period 

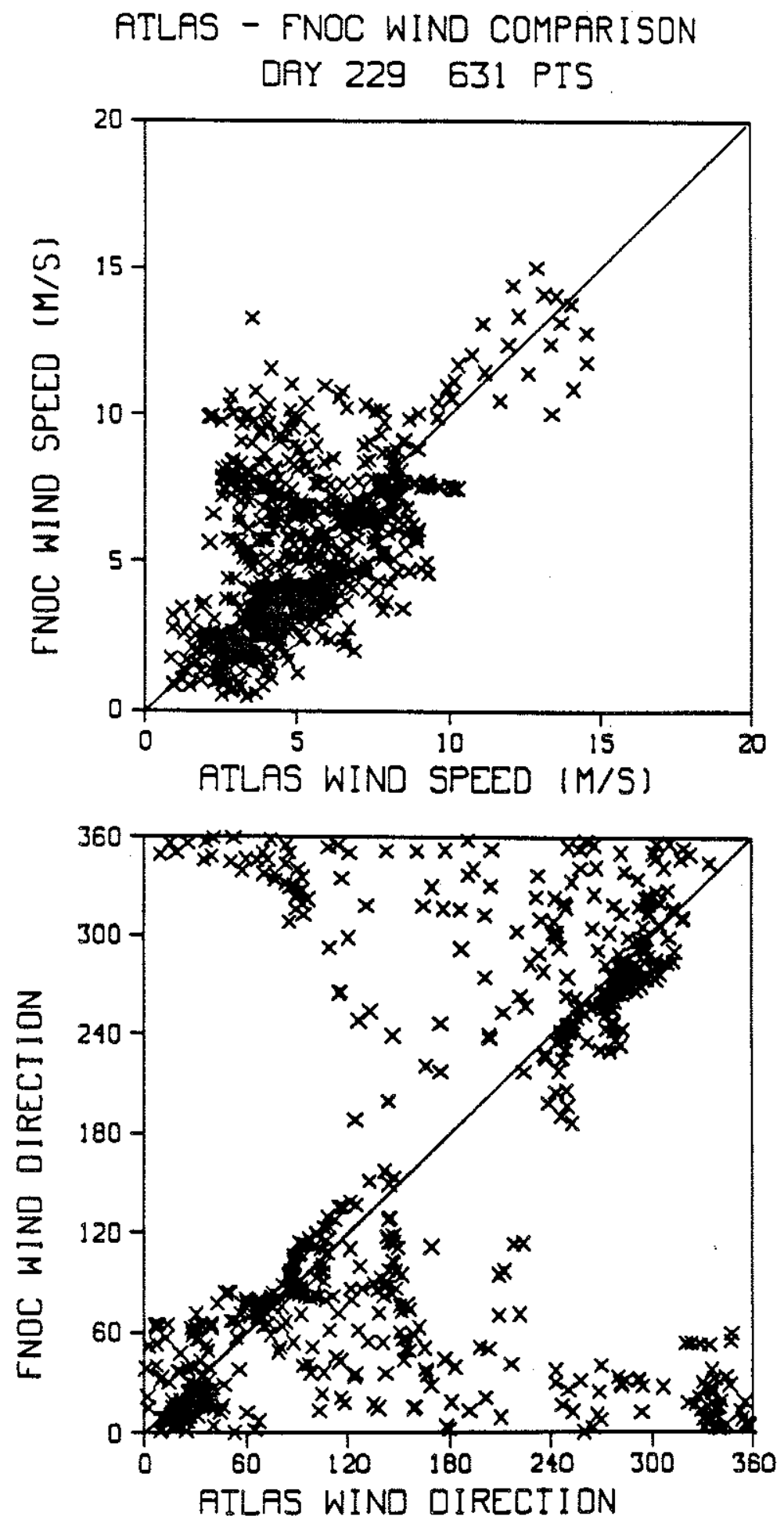

Figure 21: Mapped Atlas vs FNOC wind speed and direction - 1-day period 

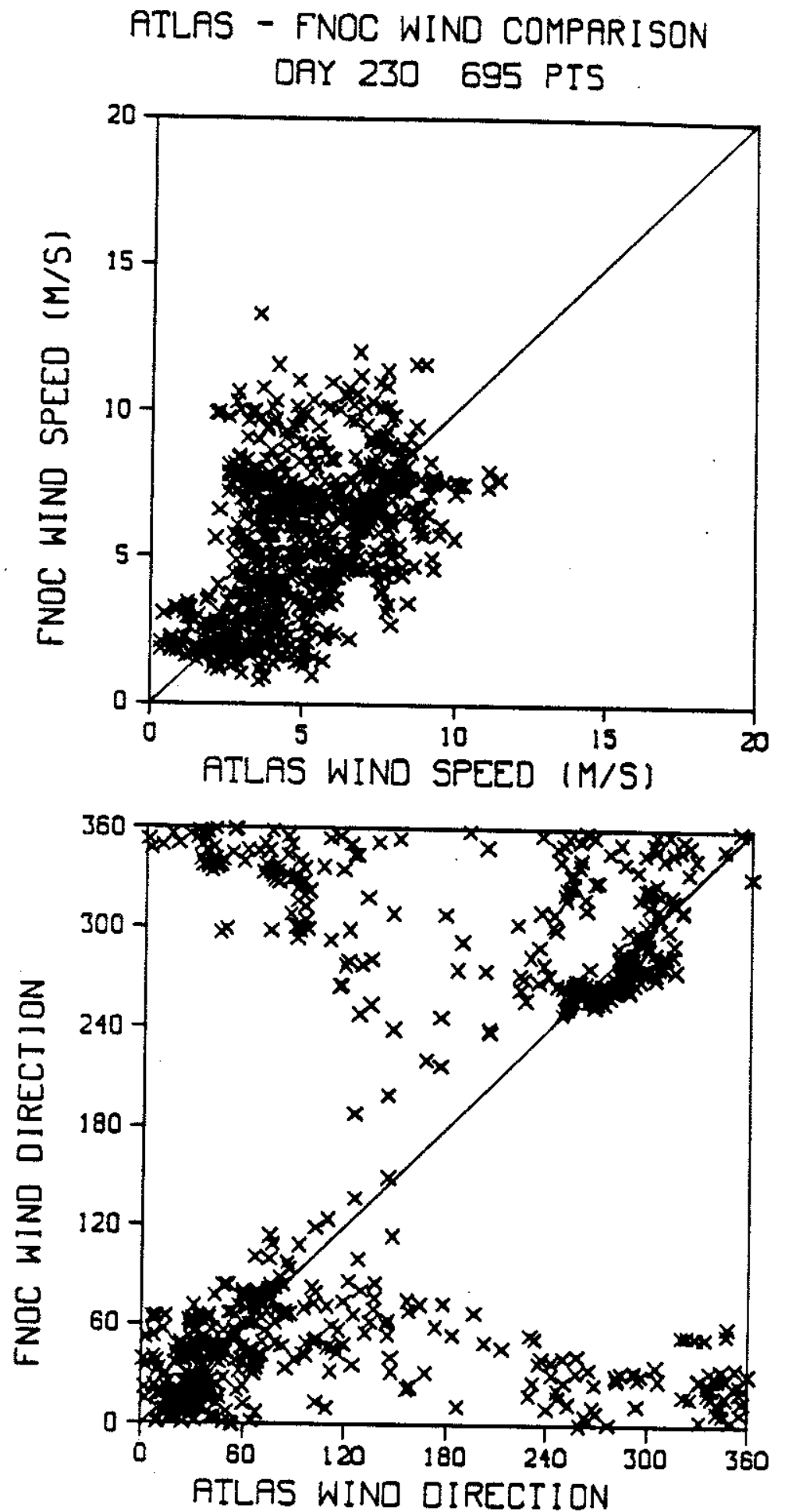

Figure 22: Mapped Atlas vs FNOC wind speed and direction - 1-day period 

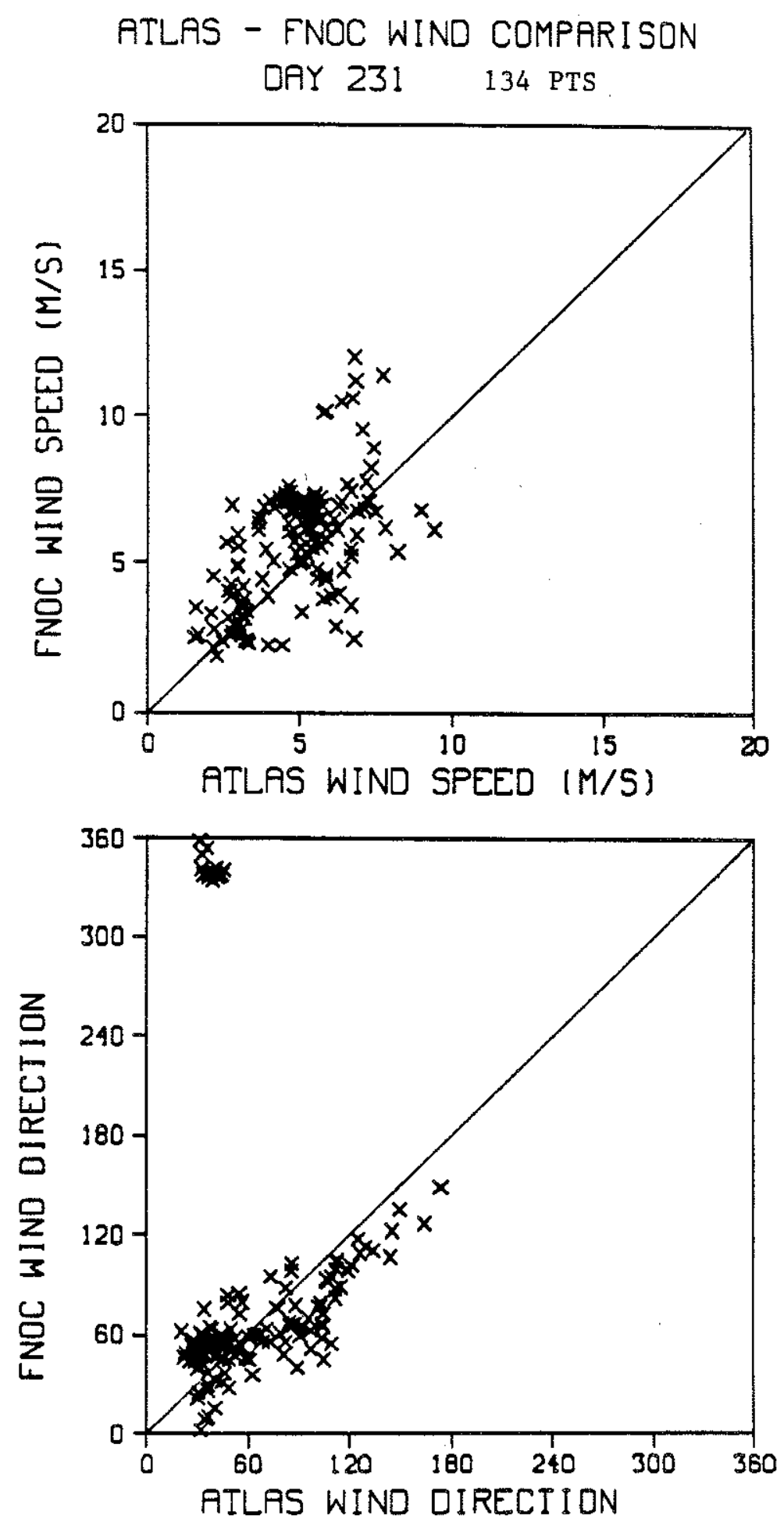

Figure 23: Mapped Atlas vs FNOC wind speed and direction - 1-day period 

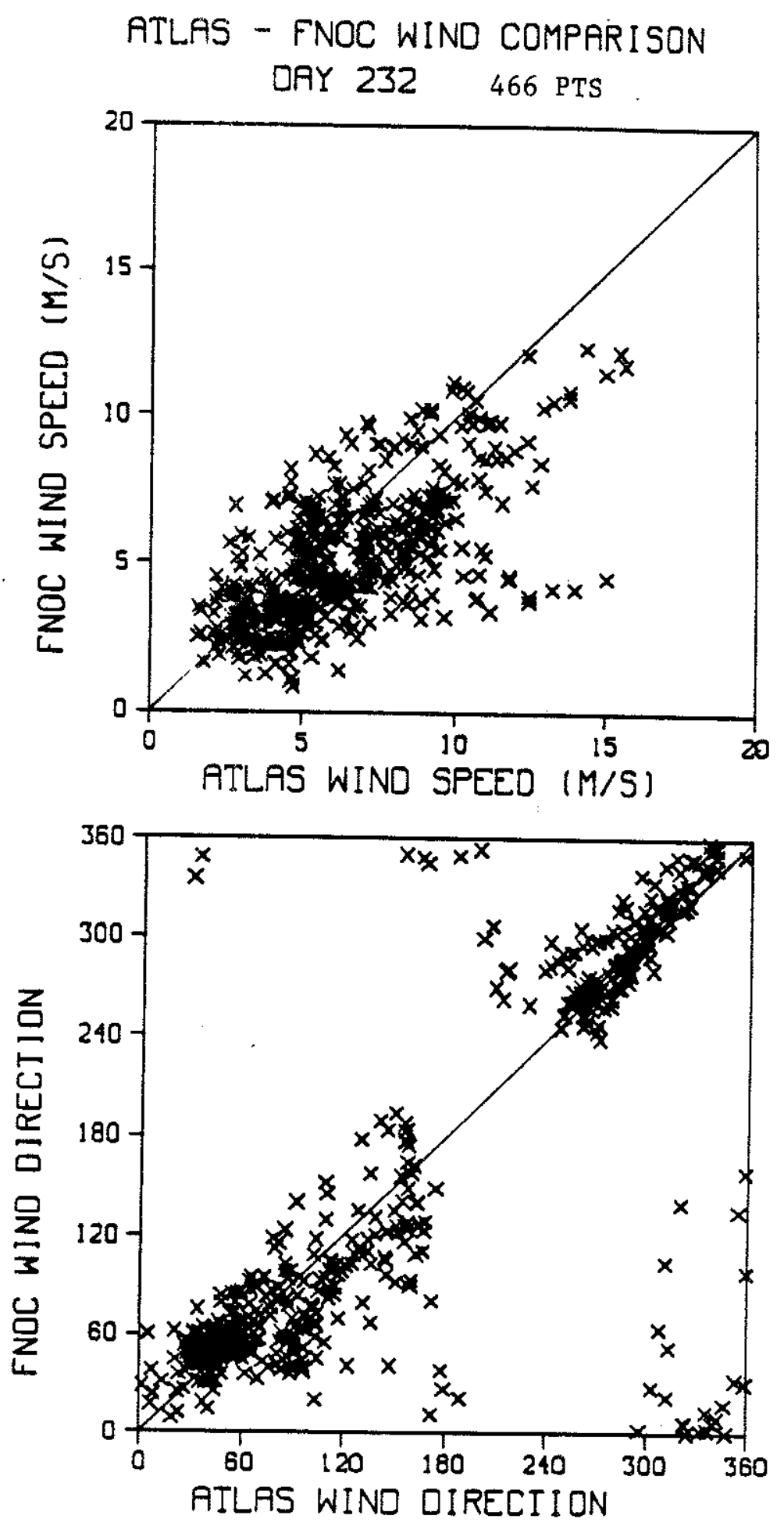

Figure 24: Mapped Atlas vs FNOC wind speed and direction - 1-day period 

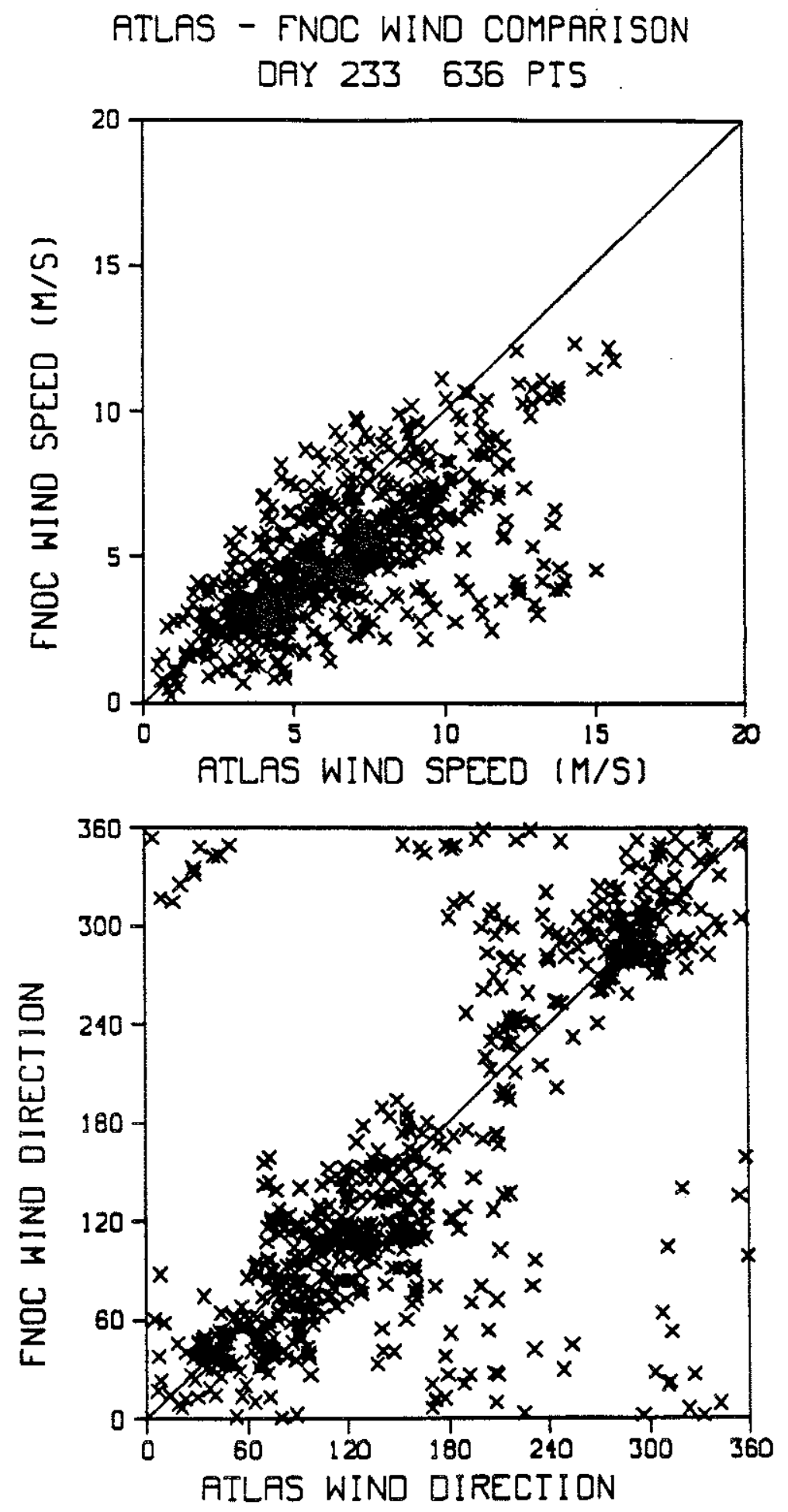

Figure 25: Mapped Atlas vs FNOC wind speed and direction - 1-day period 


\section{ATLAS - FNOC WIND COMPARISON \\ DAY 234623 PIS}
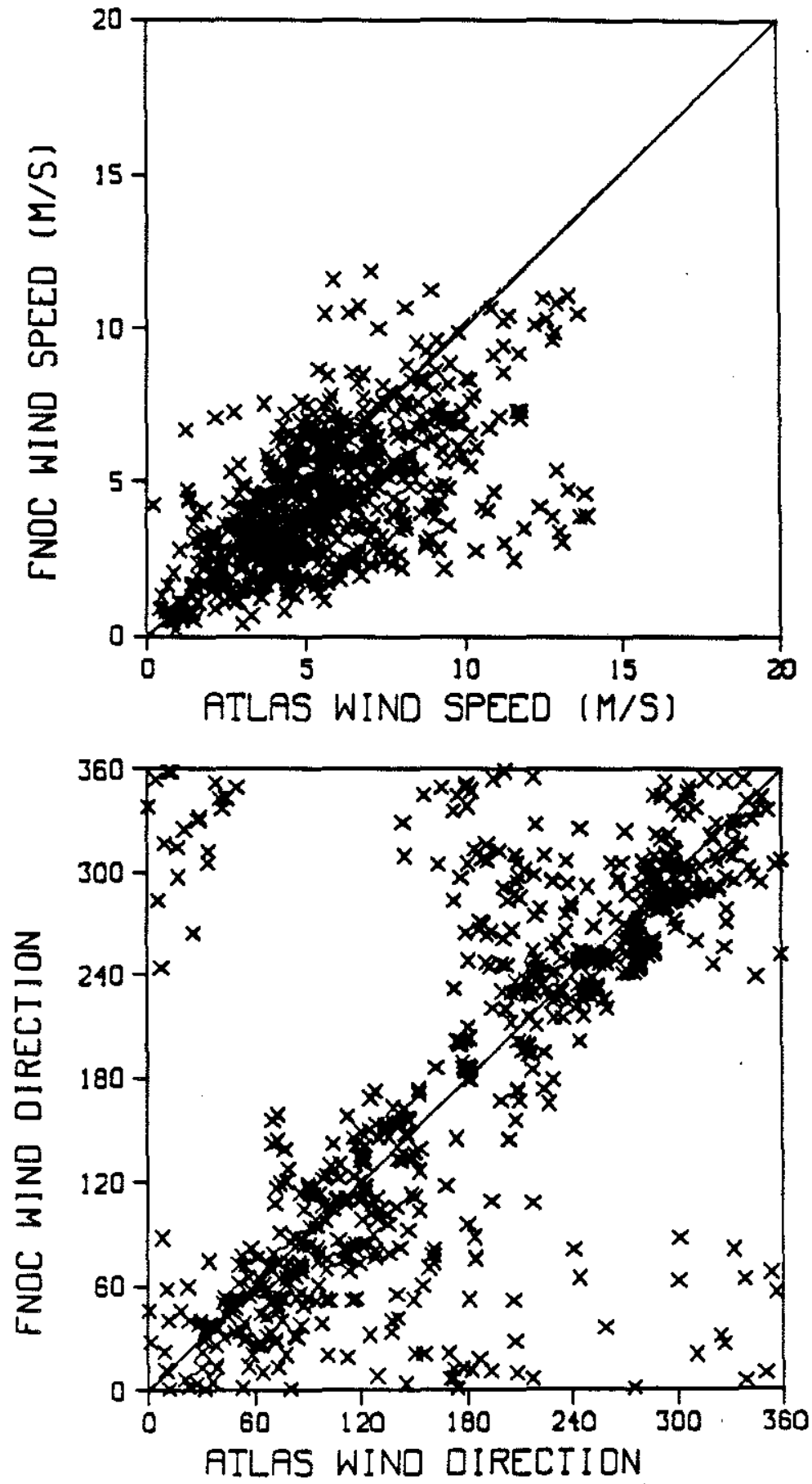

Figure 26: Mapped Atlas vs FNOC wind speed and direction - 1-day period 

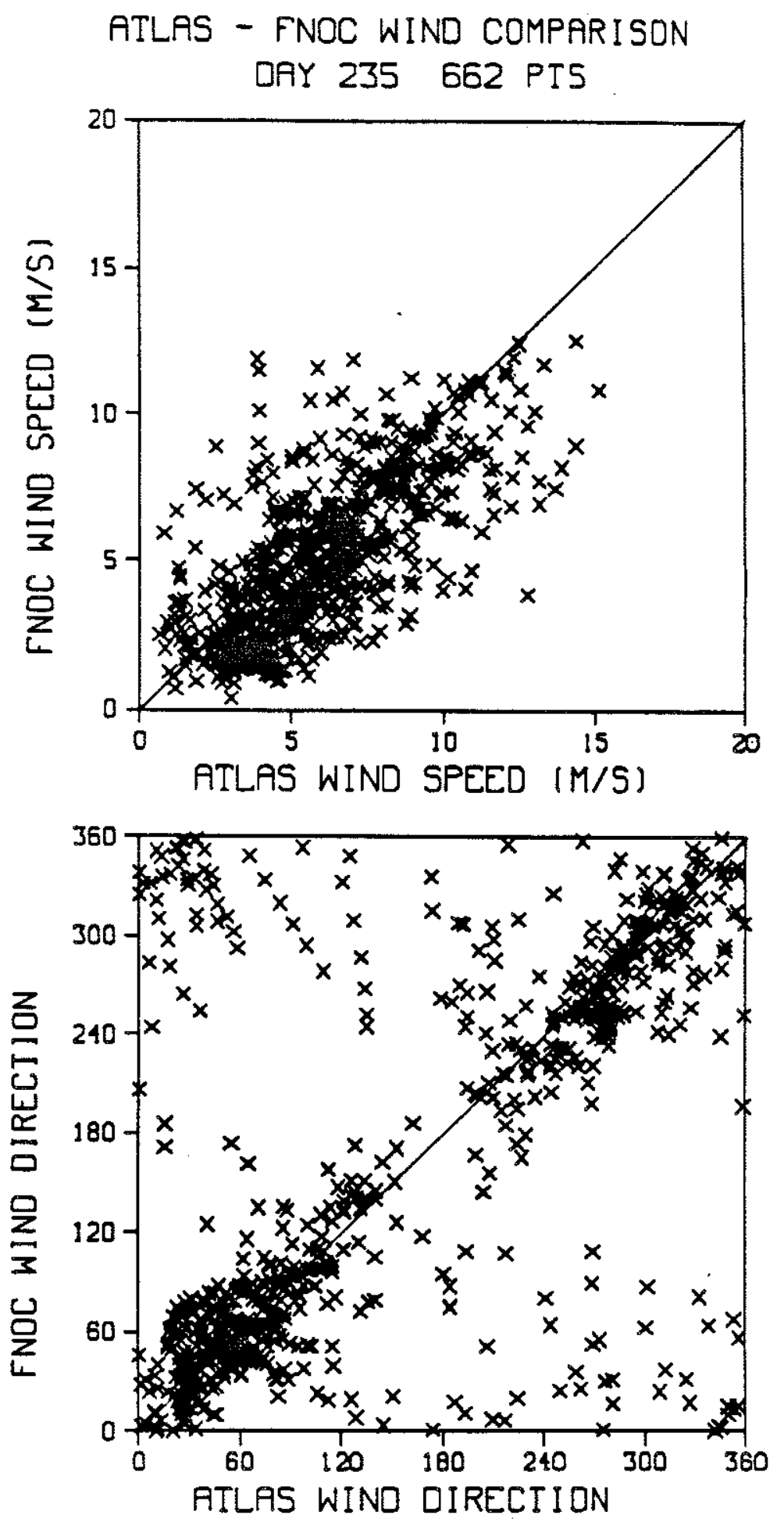

Figure 27: Mapped Atlas vs FNOC wind speed and direction - 1-day period 

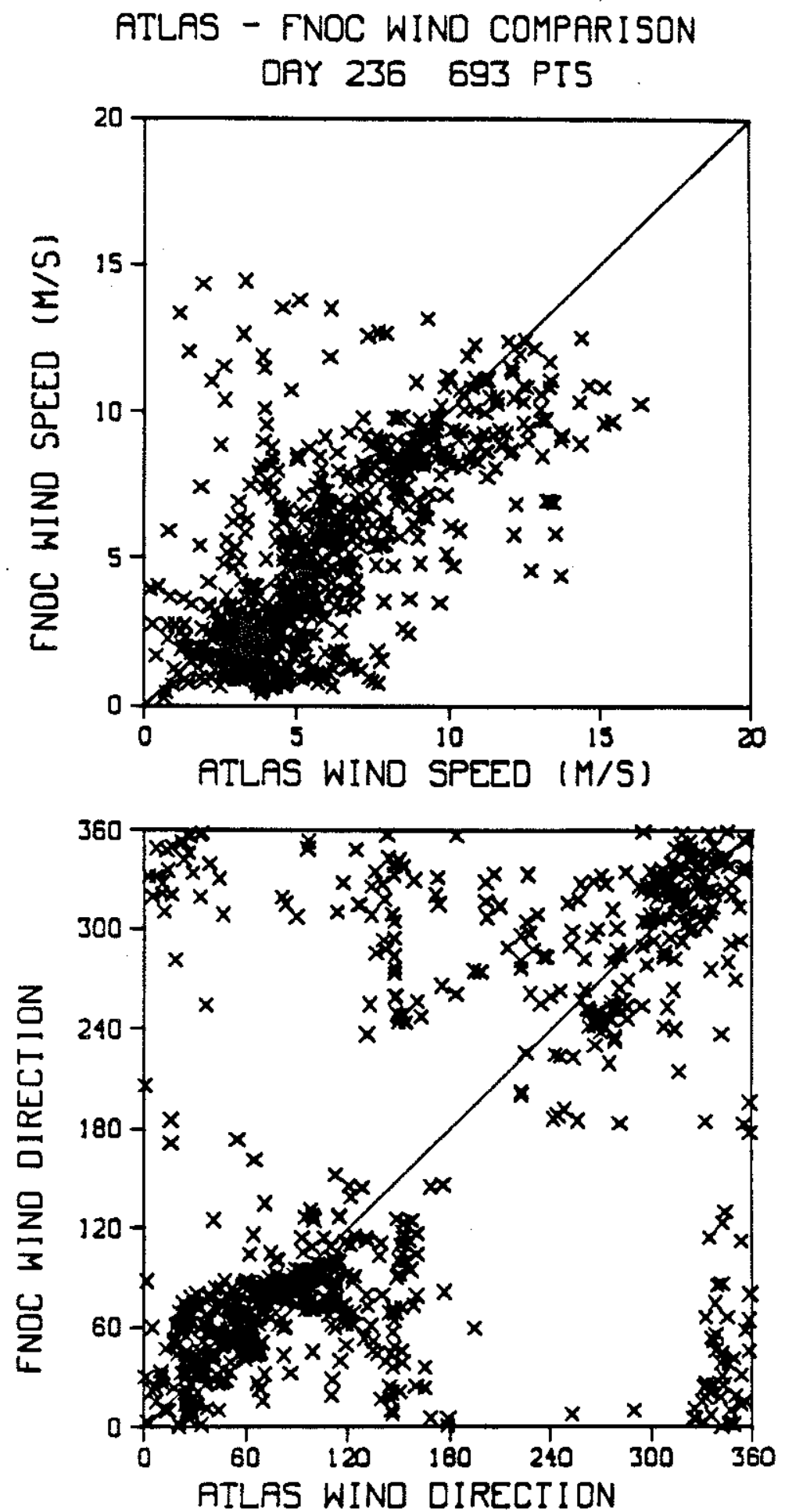

Figure 28: Mapped Atlas vs FNOC wind speed and direction - 1-day period 

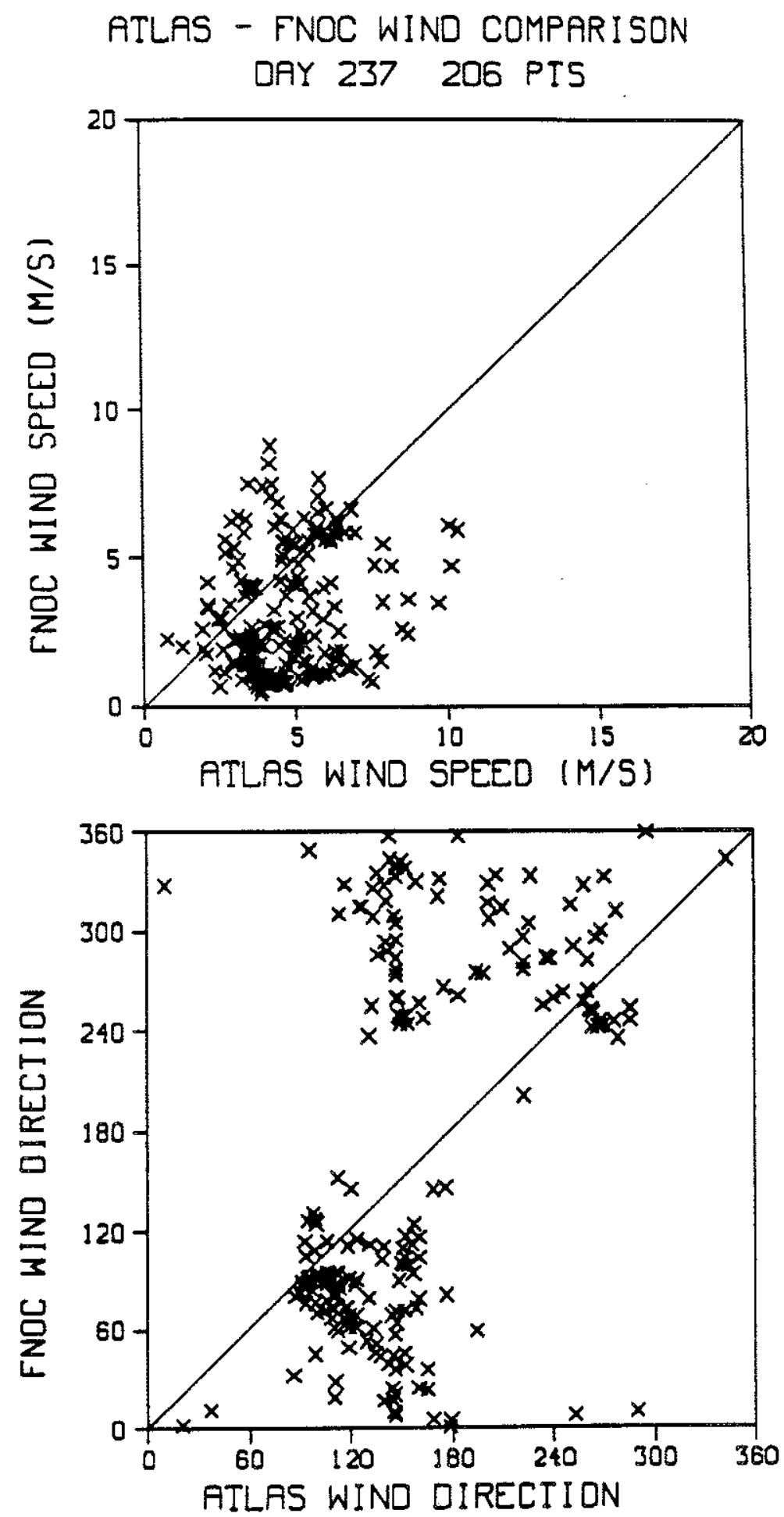

Figure 29: Mapped Atlas vs FNOC wind speed and direction - 1-day period 

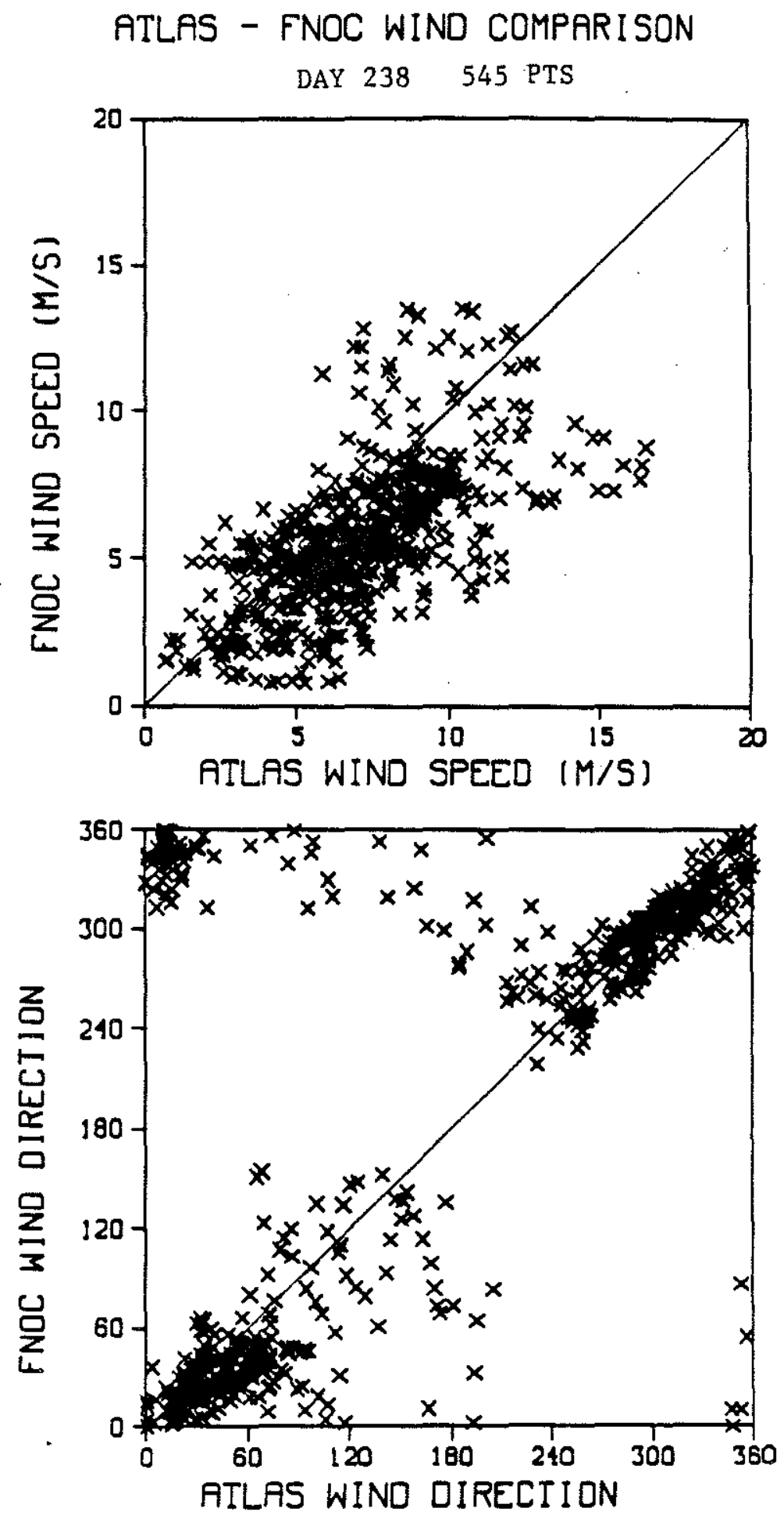

Figure 30: Mapped Atlas vs FNOC wind speed and direction - 1-day period 

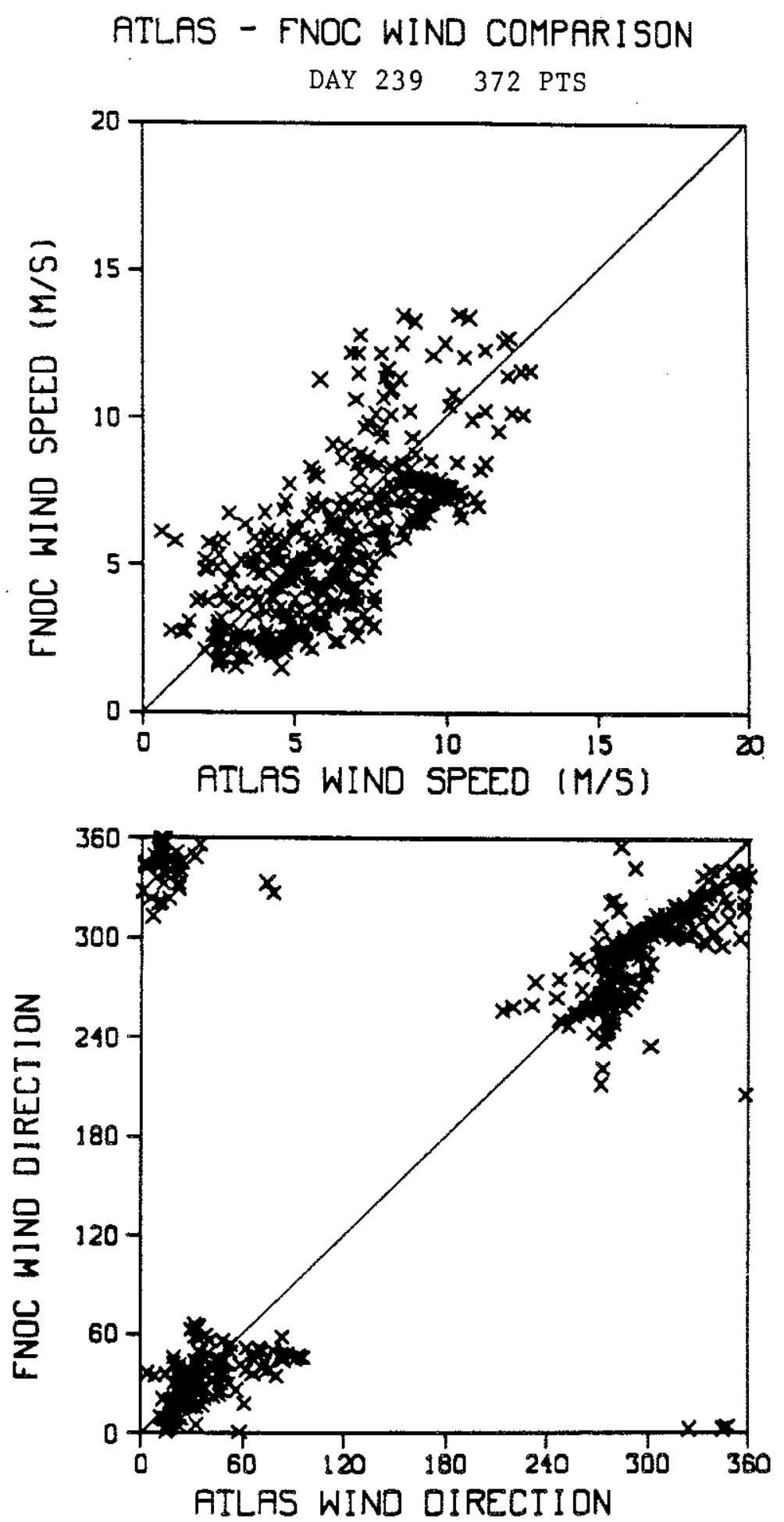

Figure 31: Mapped Atlas vs FNOC wind speed and direction - 1-day period 

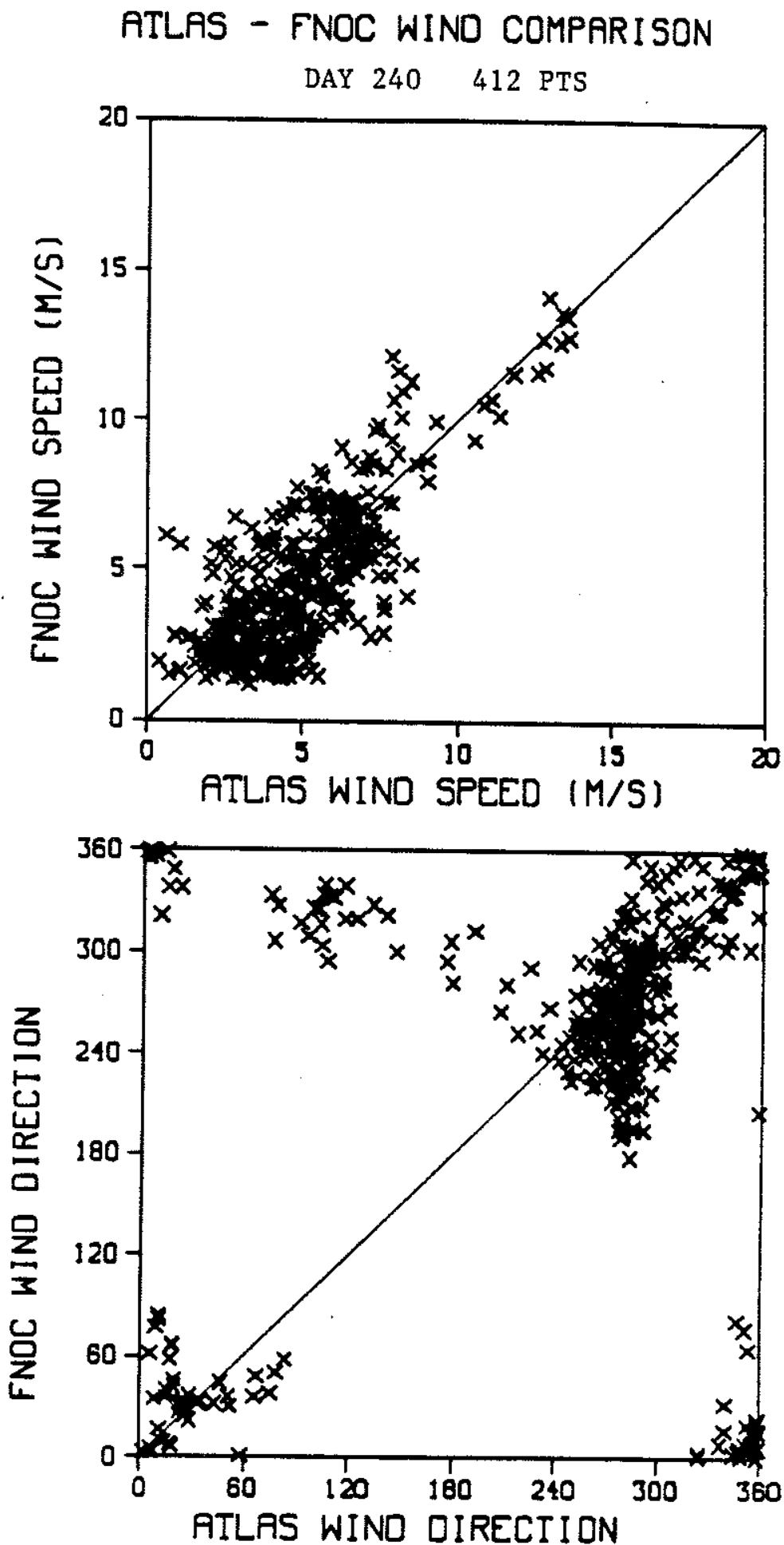

Figure 32: Mapped Atlas vs FNOC wind speed and direction - 1-day period 

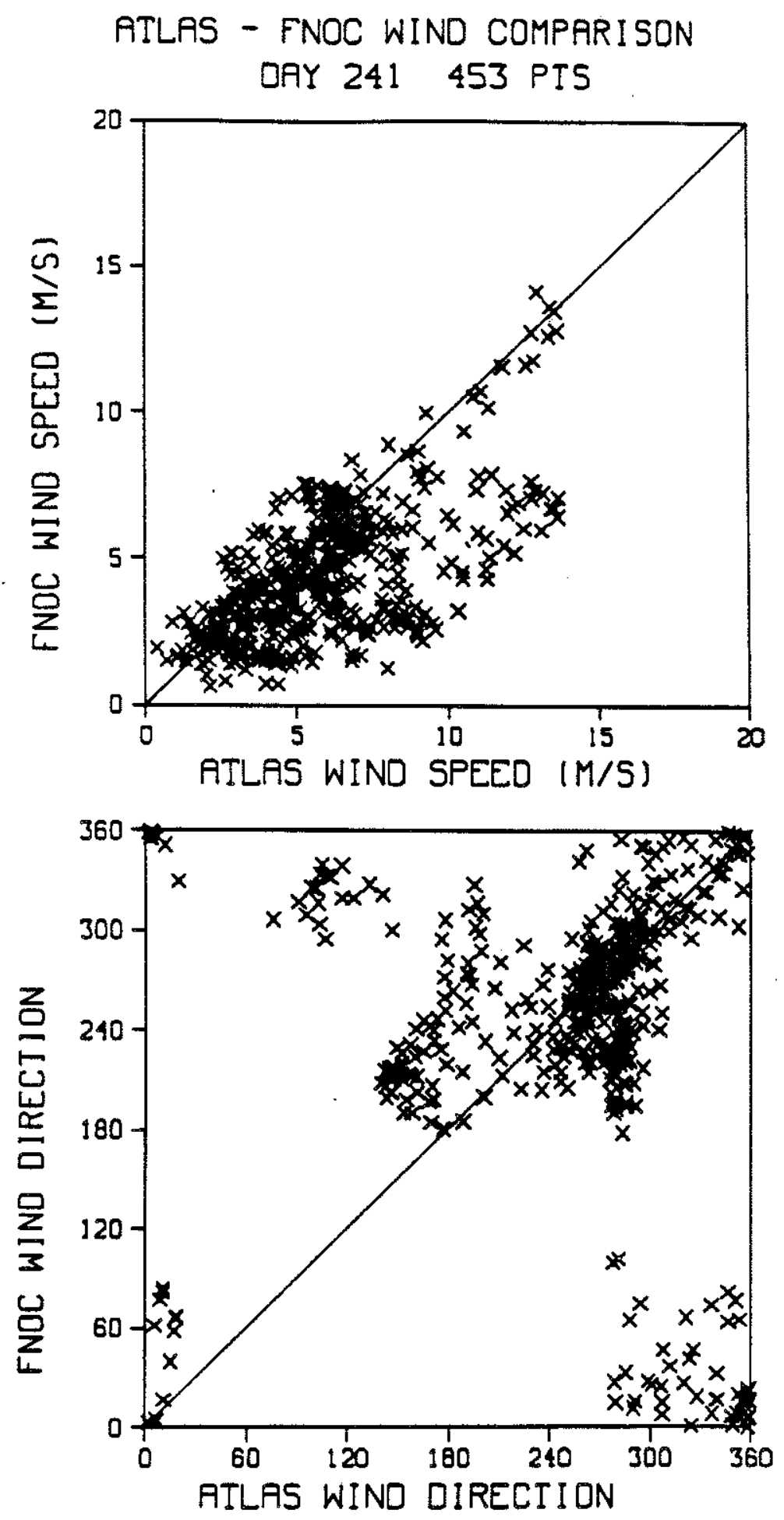

Figure 33: Mapped Atlas vs FNOC wind speed and direction - 1-day period 

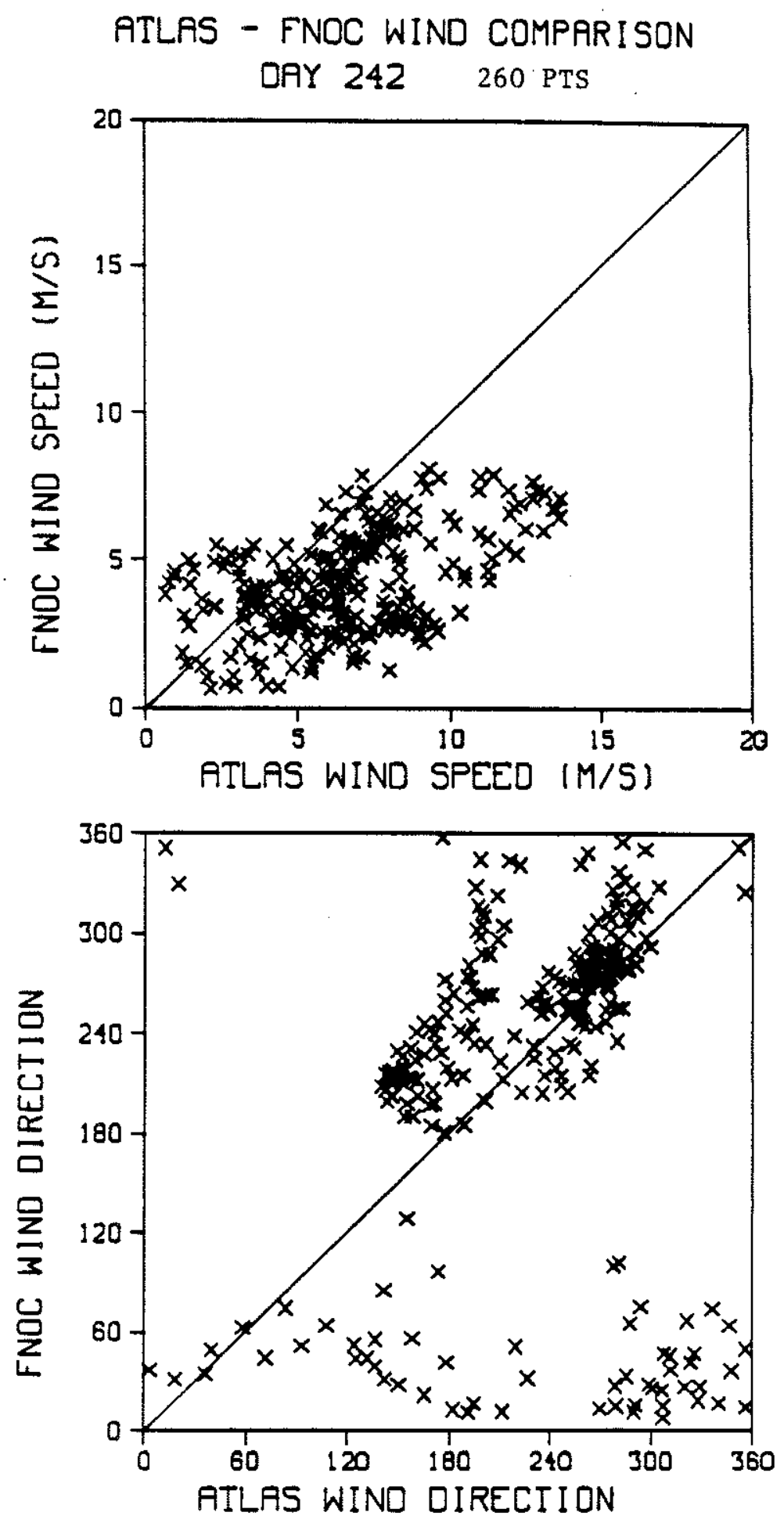

Figure 34: Mapped Atlas vs FNOC wind speed and direction - 1-day period 

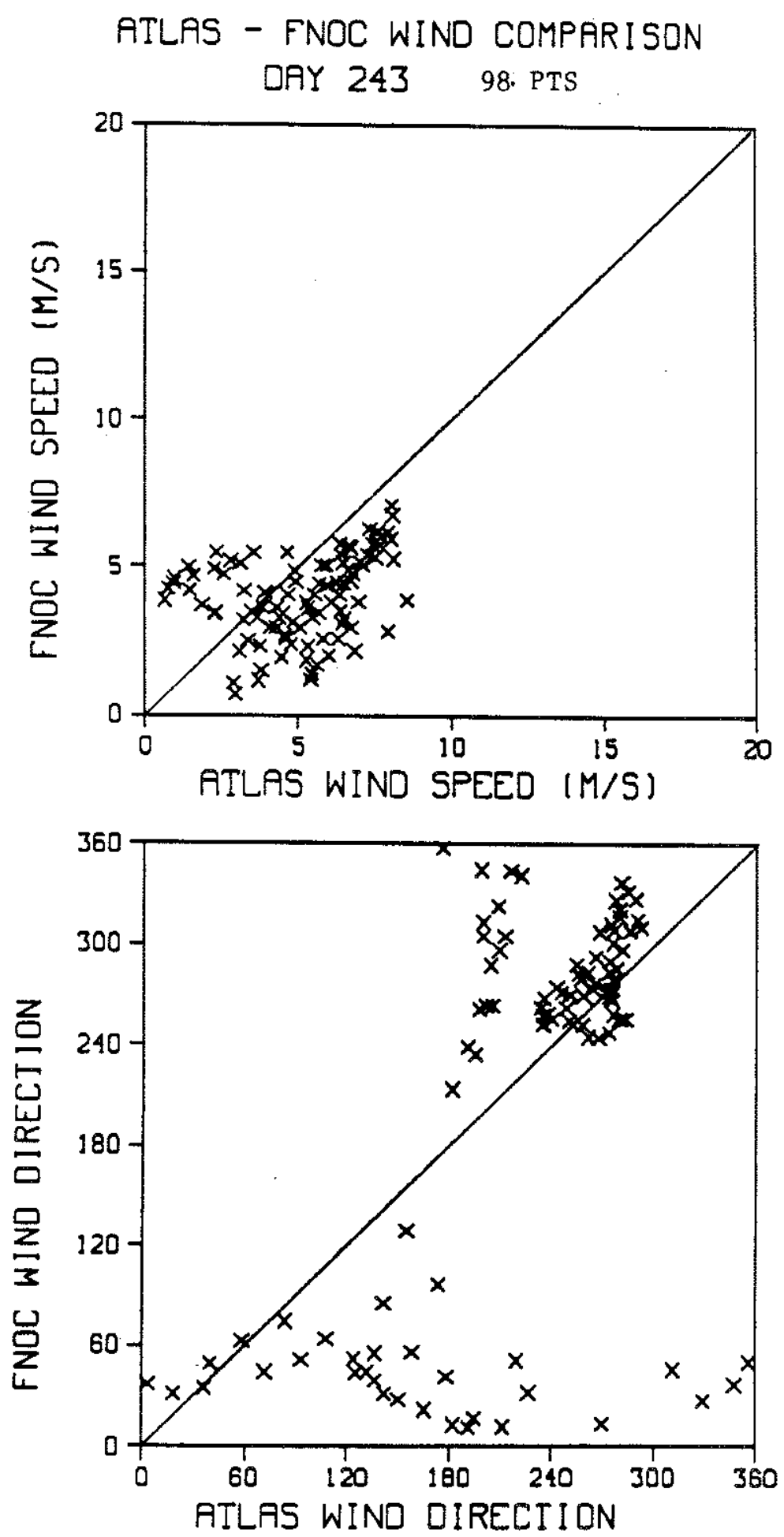

Figure 35: Mapped Atlas vs FNOC wind speed and direction - 1-day period 

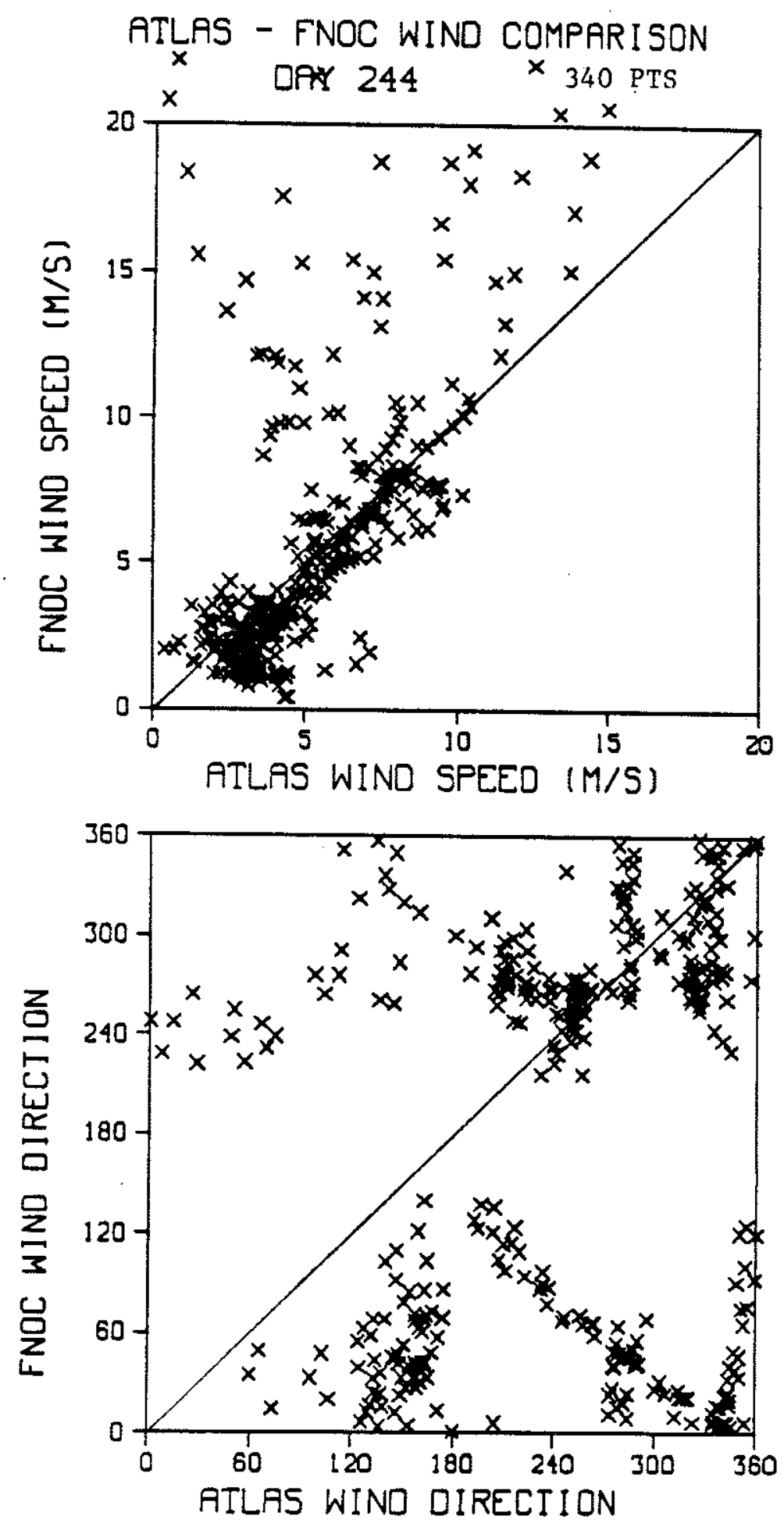

Figure 36: Mapped Atlas vs FNOC wind speed and direction - 1-day period 

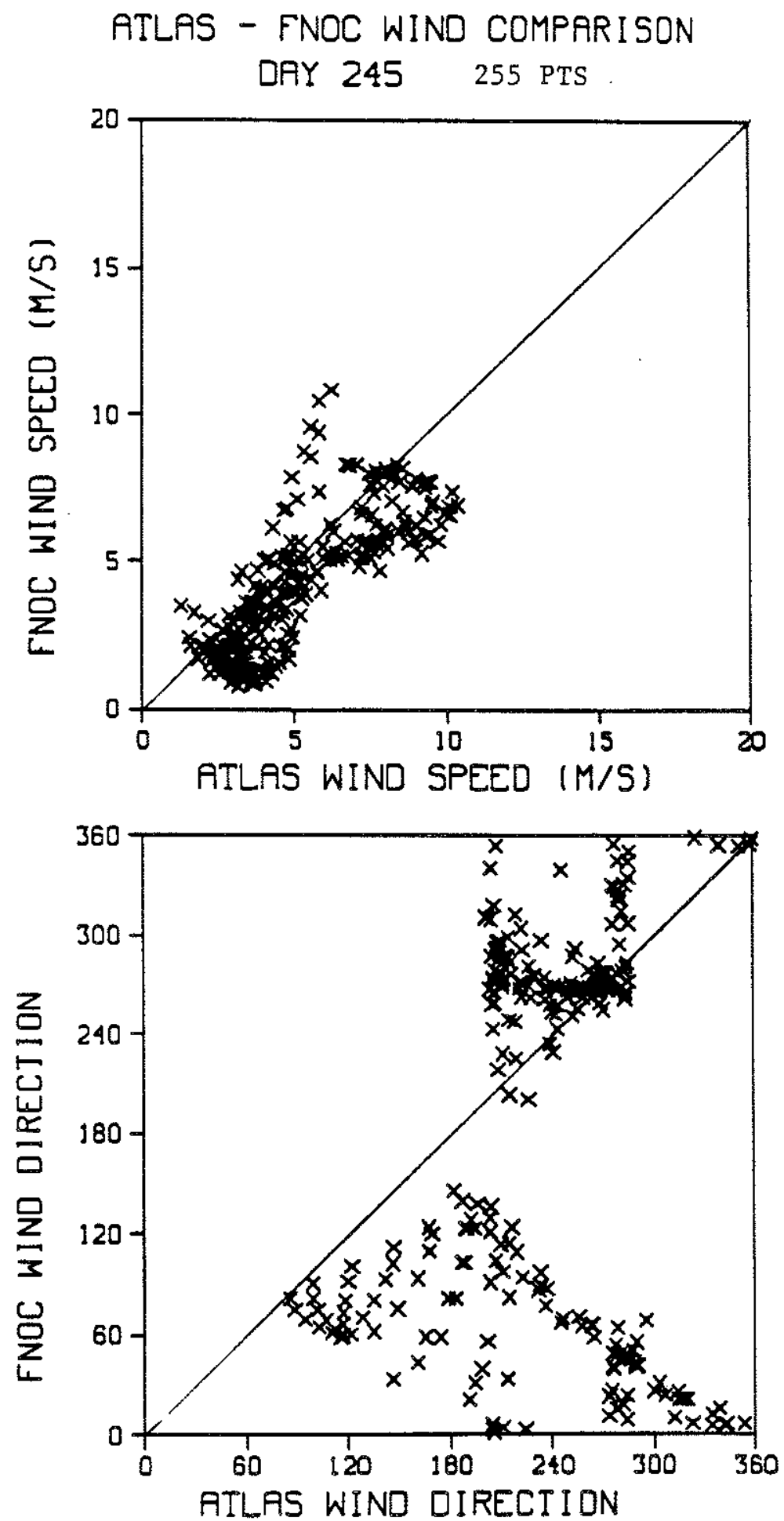

Figure 37: Mapped Atlas vs FNOC wind speed and direction - 1-day period 

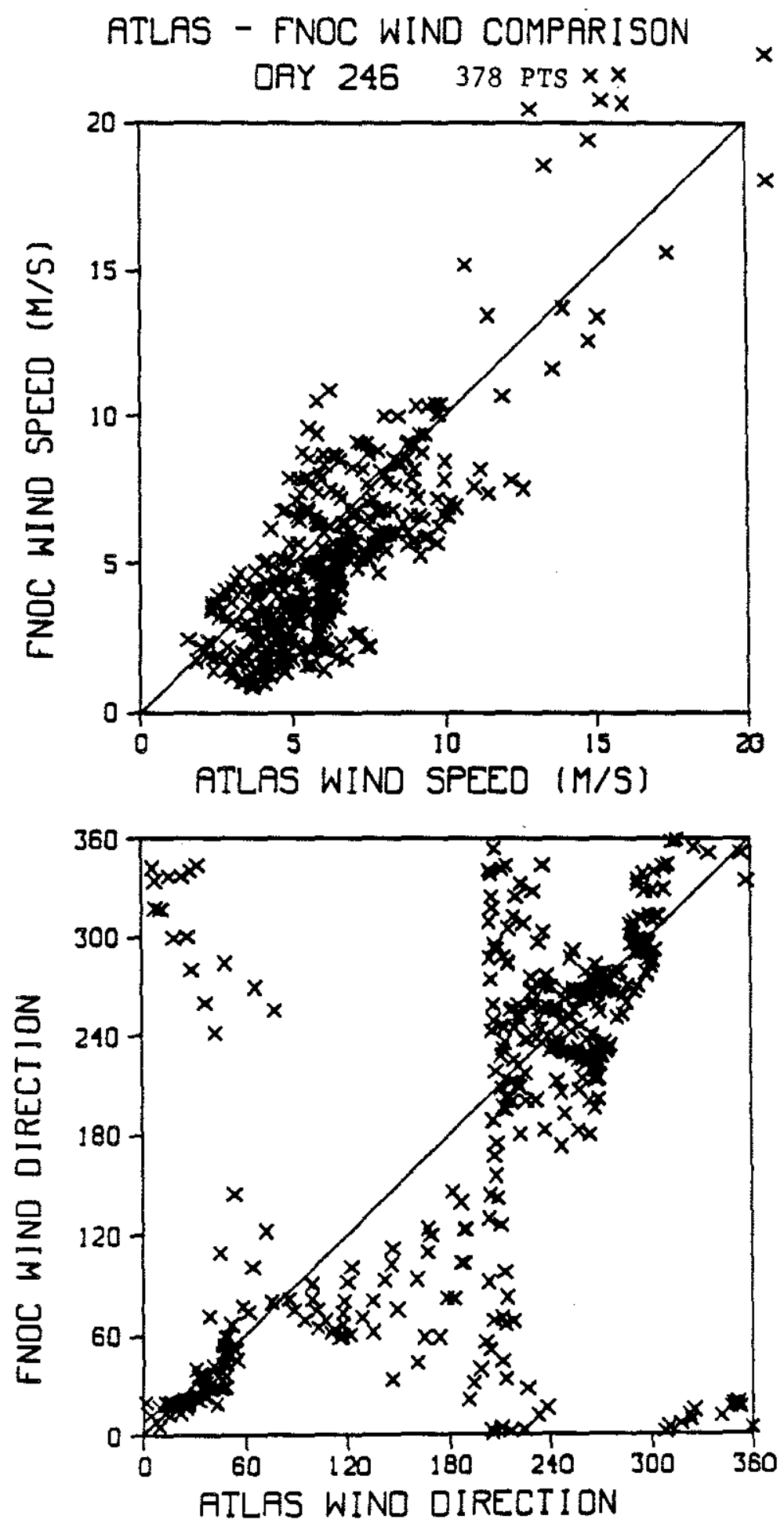

Figure 38: Mapped Atlas vs FNOC wind speed and direction - 1-day period 

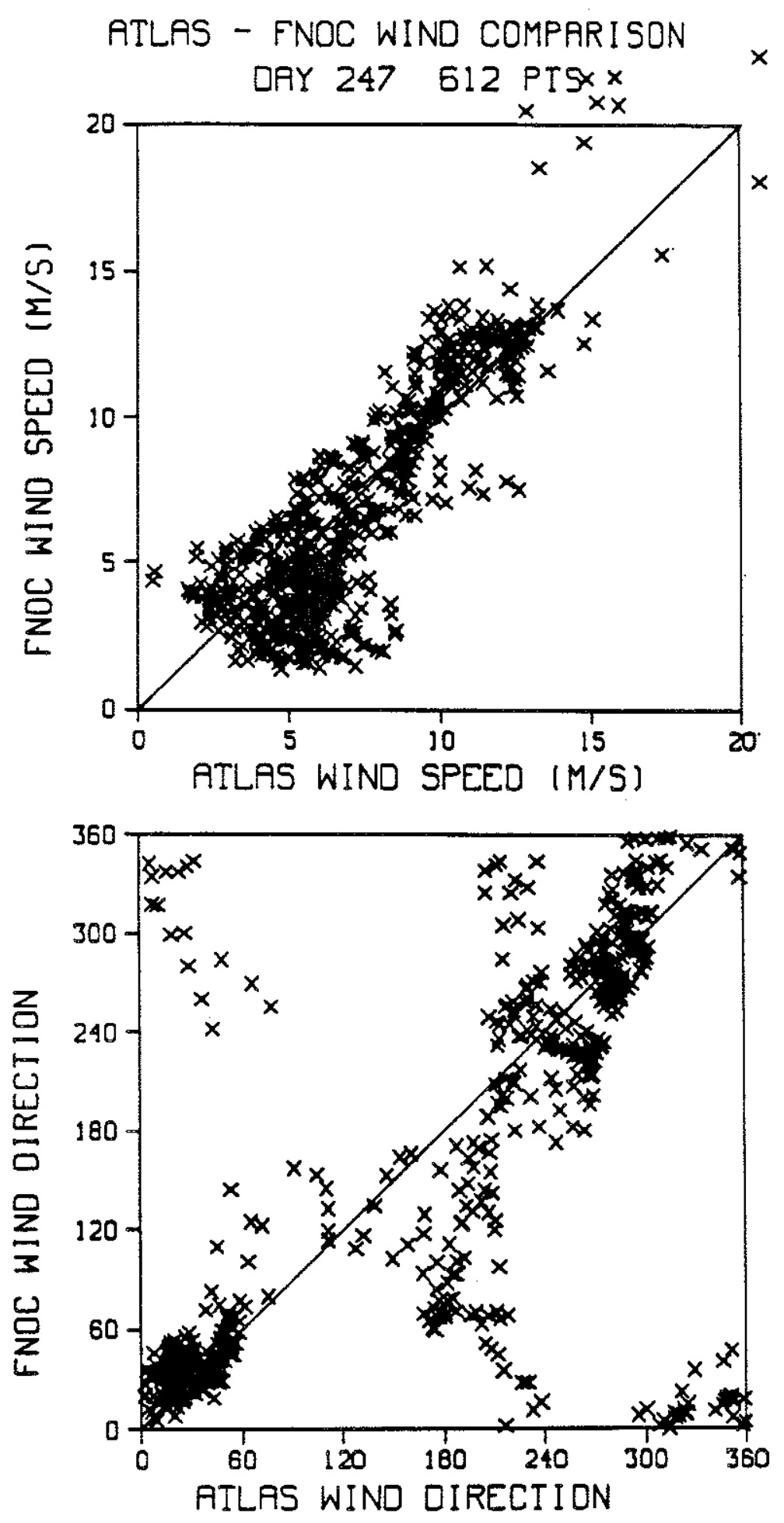

Figure 39: Mapped Atlas vs FNOC wind speed and direction - 1-day period 

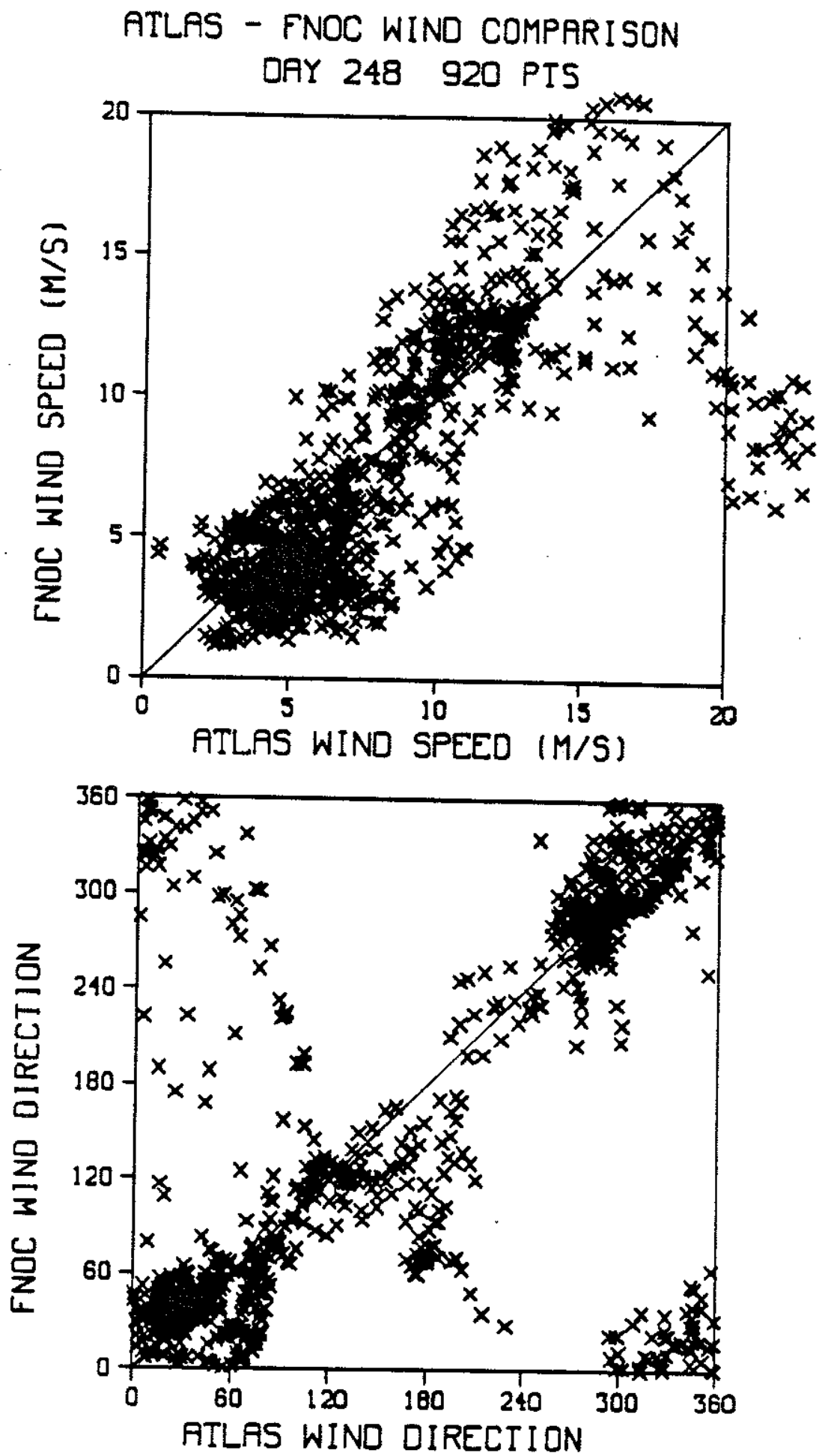

Figure 40: Mapped Atlas vs FNOC wind speed and direction - 1-day period 

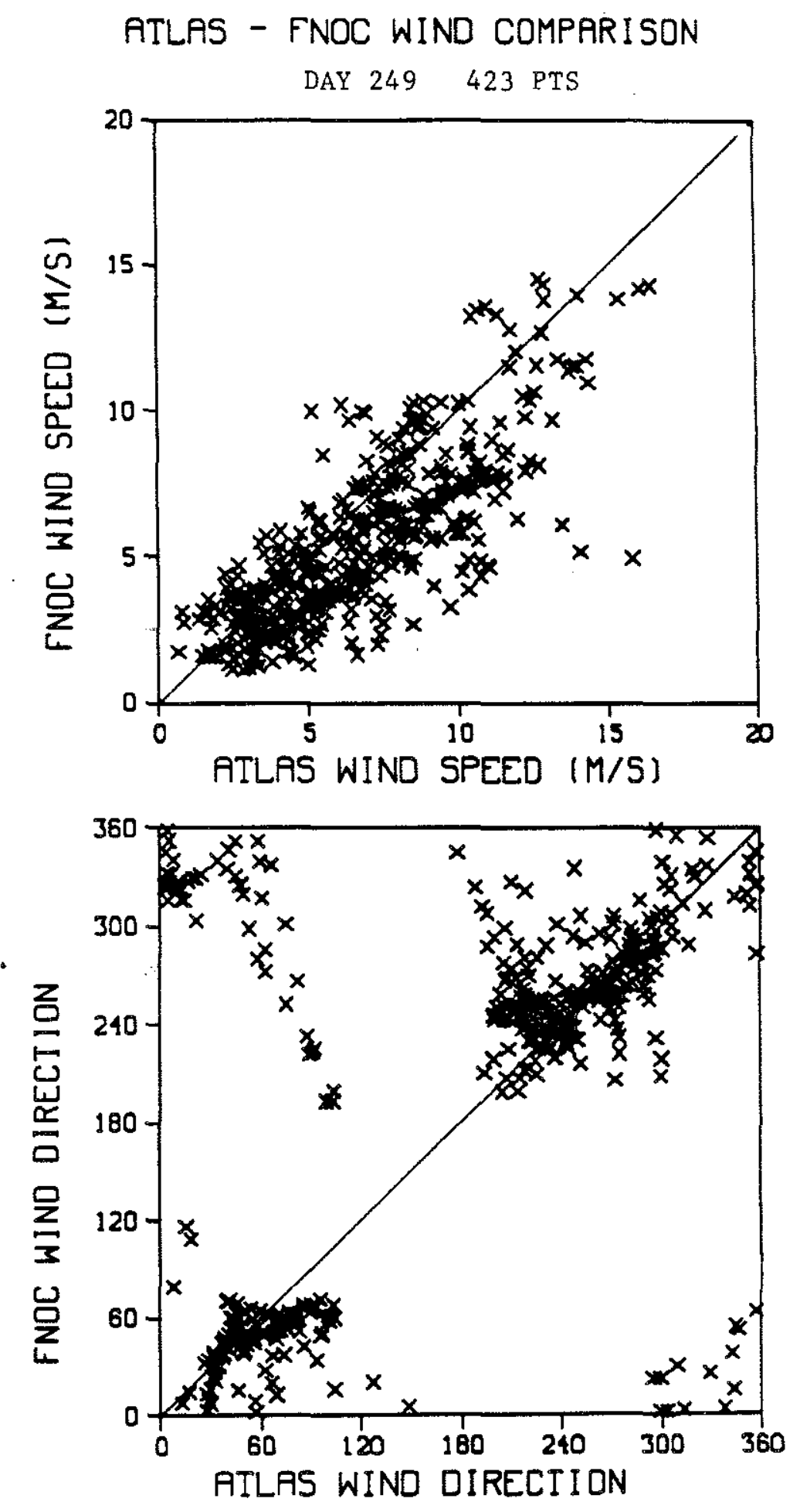

Figure 41: Mapped Atlas vs FNOC wind speed and direction - 1-day period 

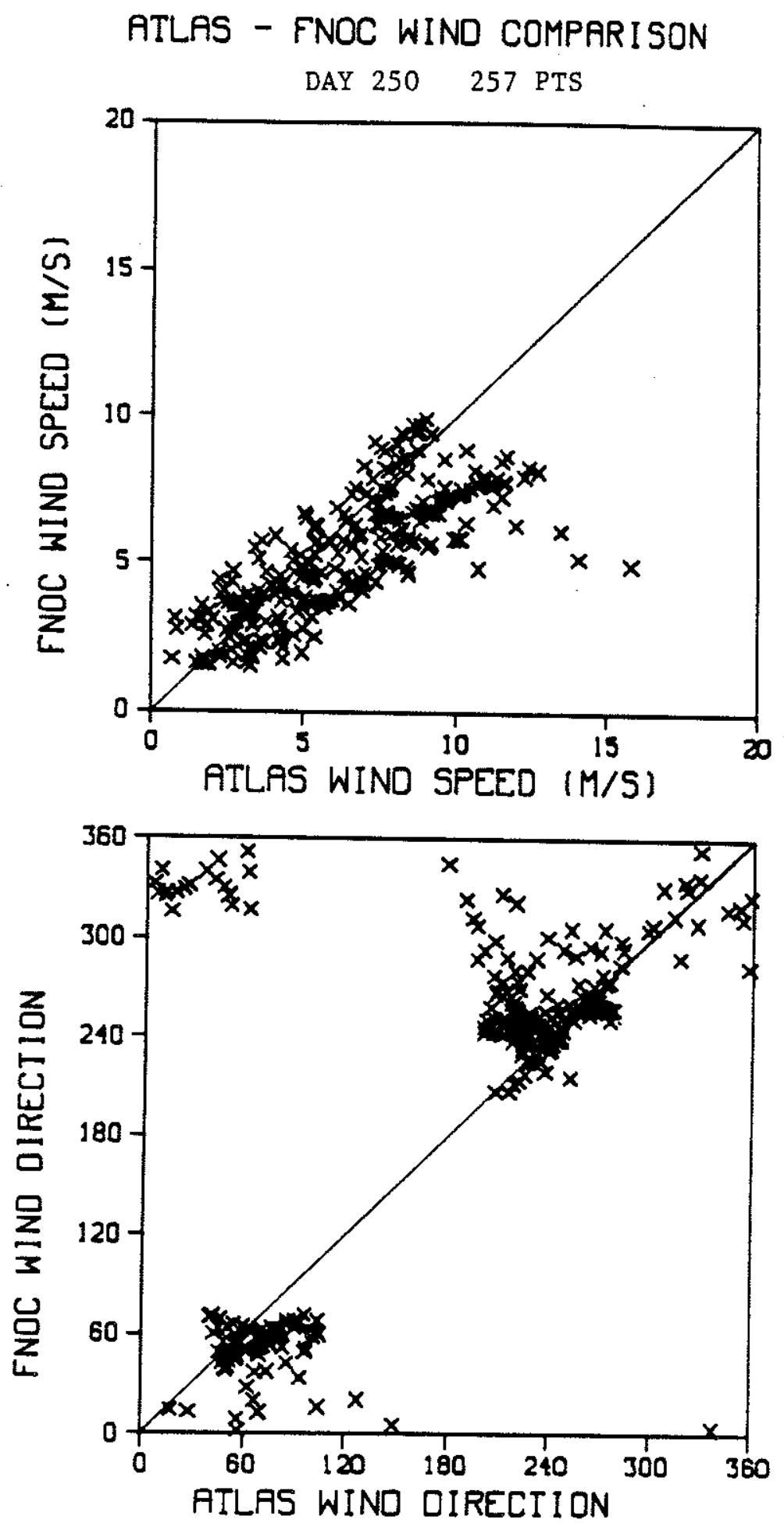

Figure 42: Mapped Atlas vs FNOC wind speed and direction - 1-day period 
ATLAS - FNOC WIND COMPARISON

DAY 251404 PTS
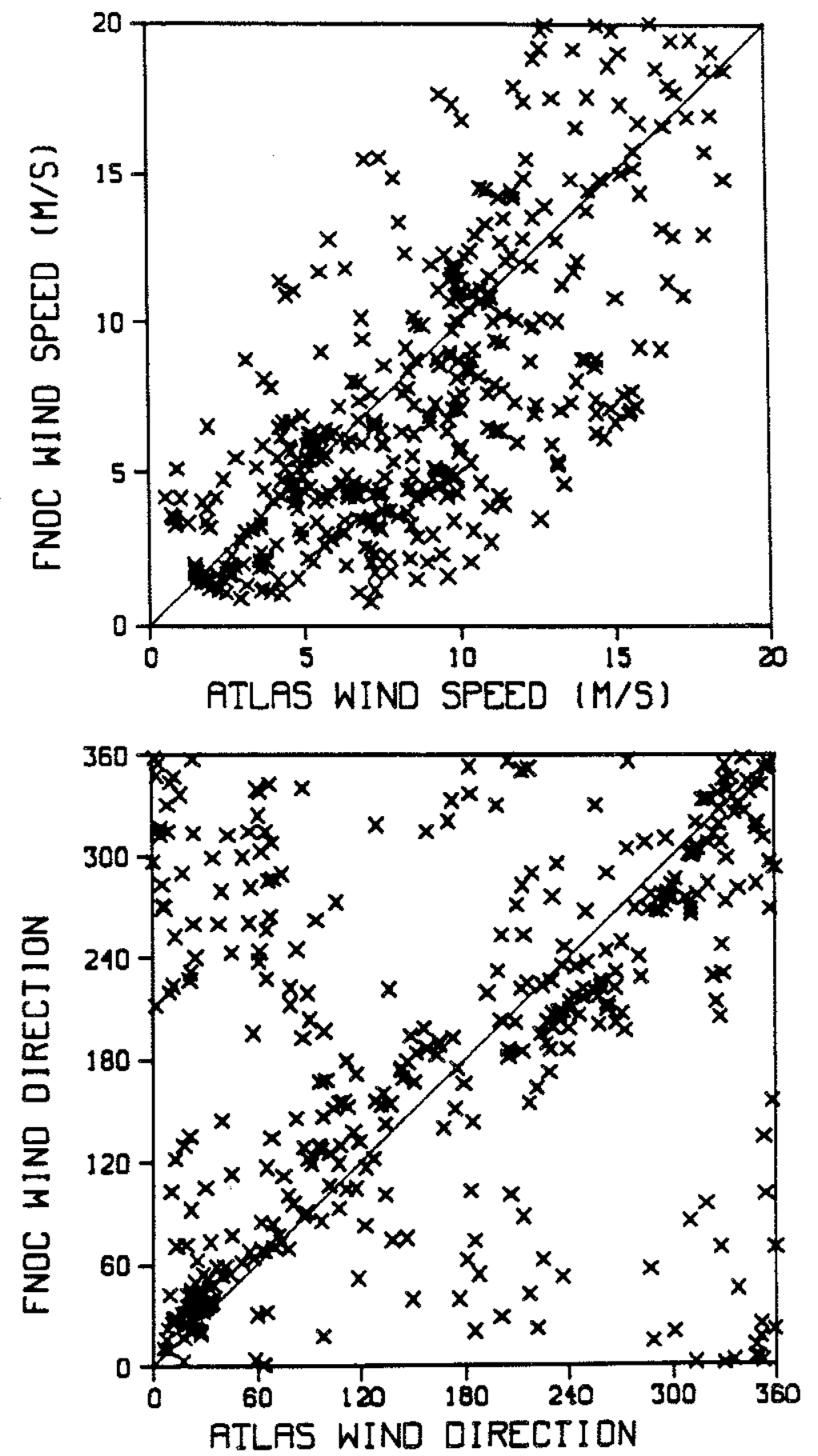

Figure 43: Mapped Atlas vs FNOC wind speed and direction - 1-day period 


\section{ATLAS - FNOC WIND COMPARISON}

DAY $252 \quad 317$ PTS
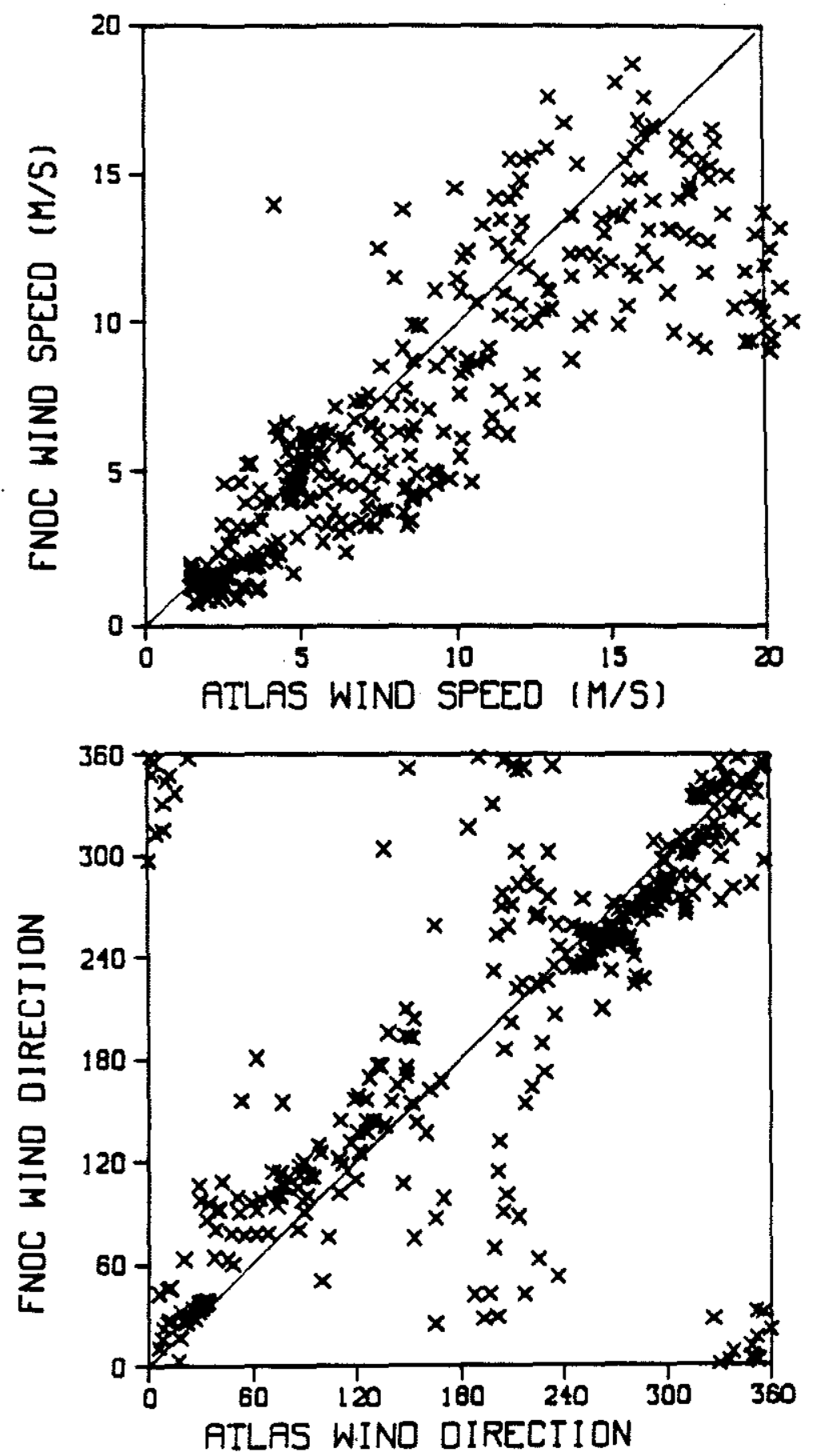

Figure 44: Mapped Atlas vs FNOC wind speed and direction - 1-day period 
ATLRS SCATIEROMEIER WINDS

REV 1008

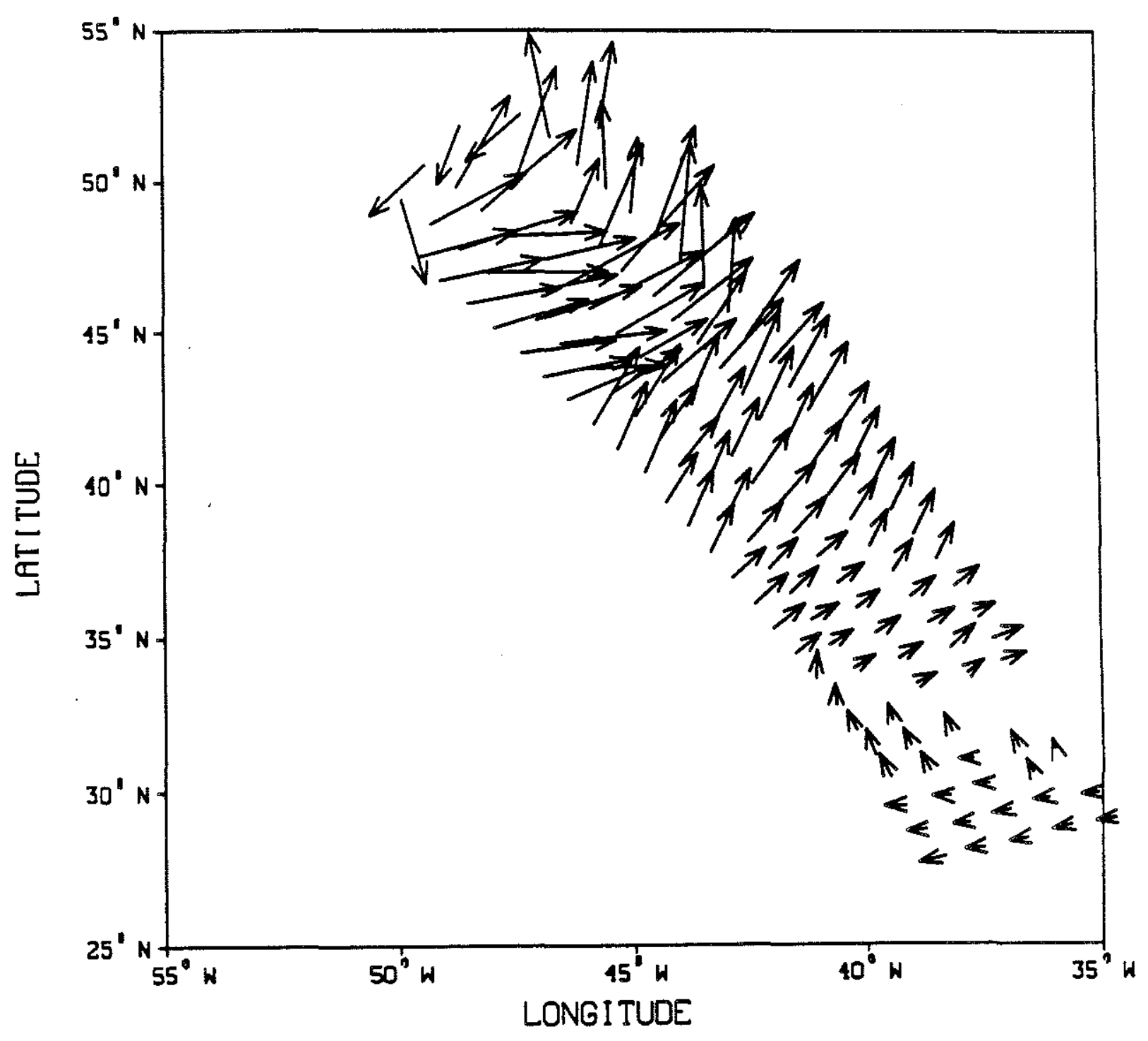

Figure 45: Ungridded Atlas winds - Rev 1008 
FNOC SYNOPIIC WINDS

248122

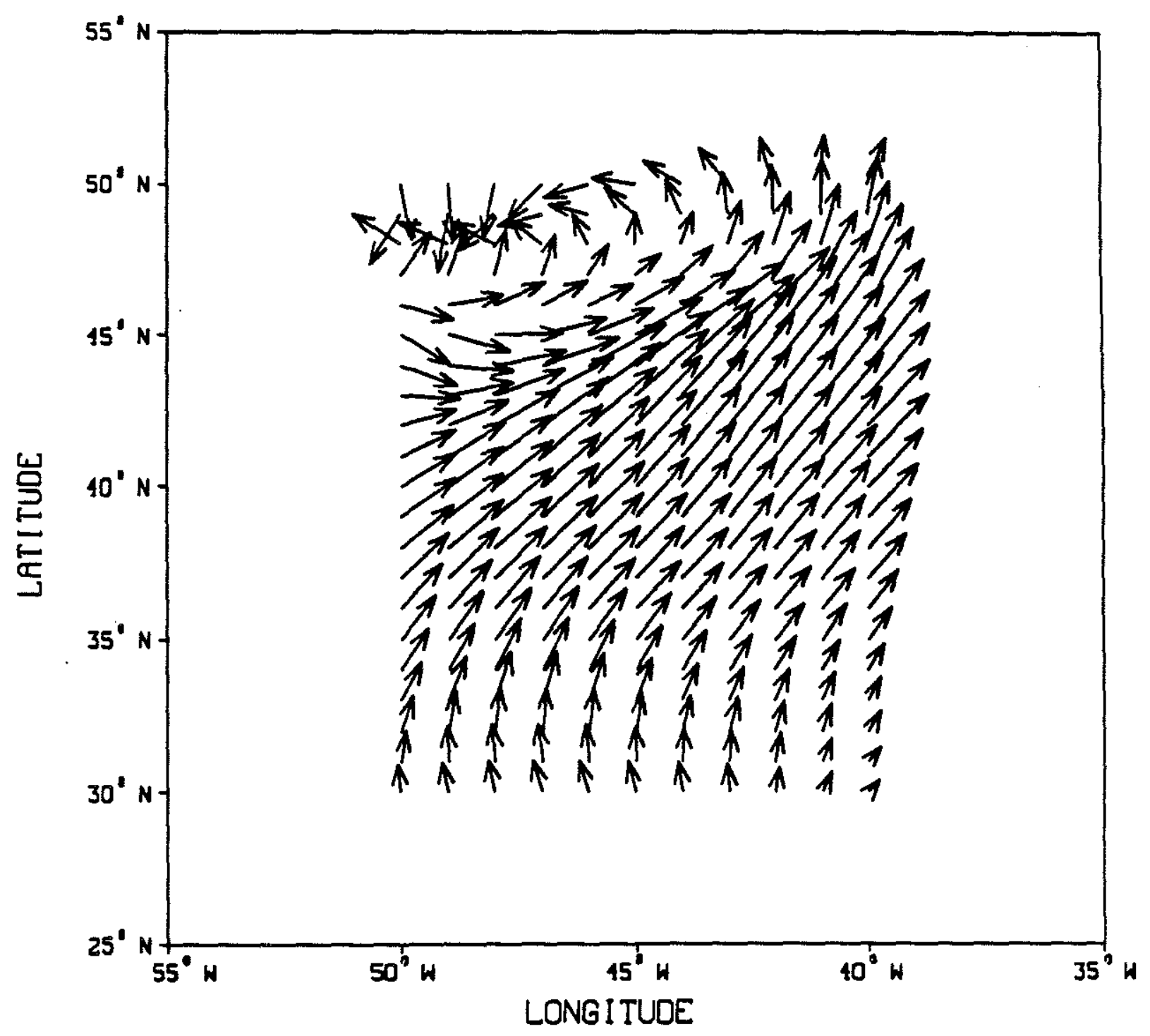

Figure 46: FNOC winds - Day 248122 


\section{ATLRS VS FNOC SCATTER PLOT \\ 1 DAY MEAN SPEED}

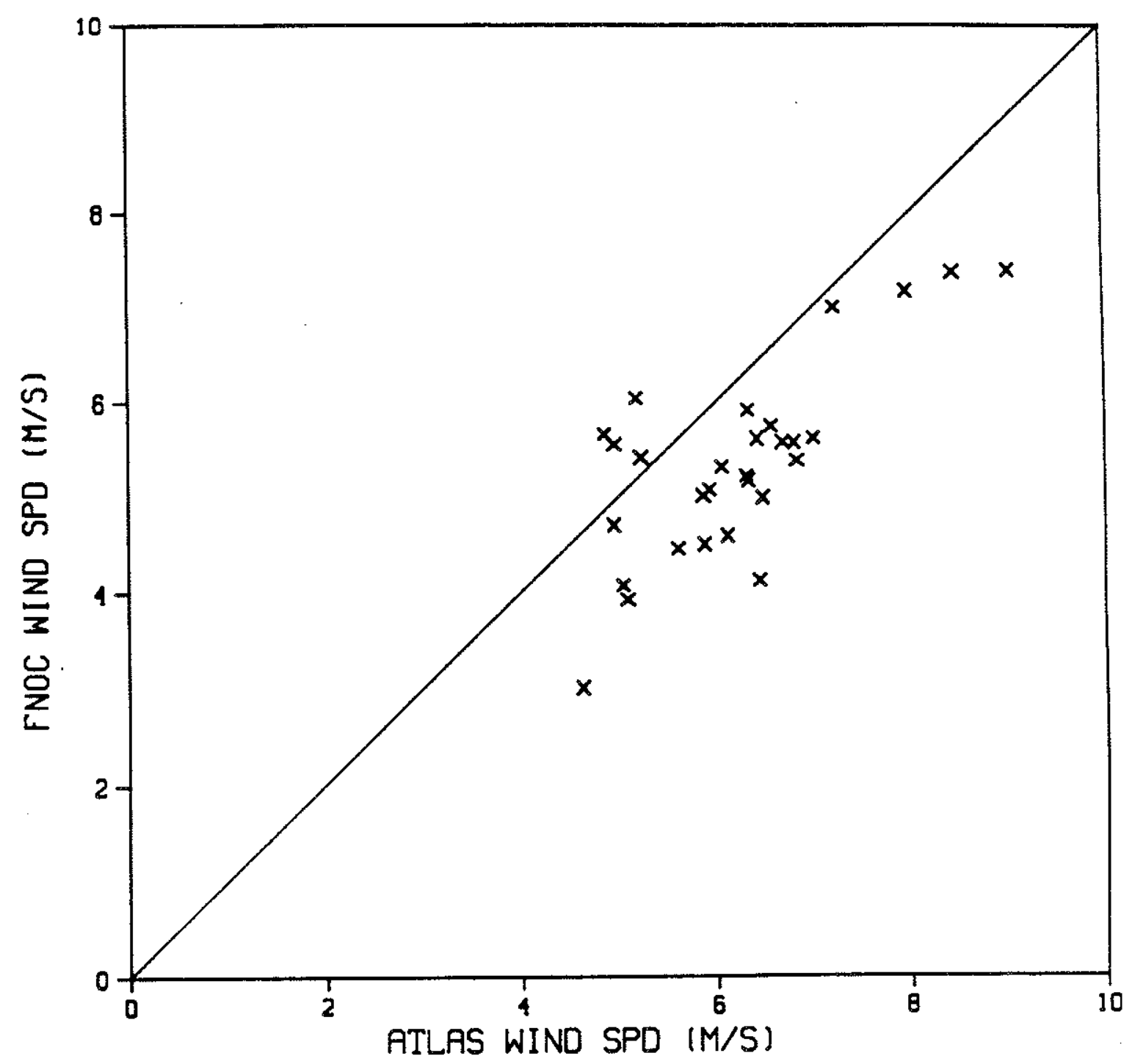

Figure 47: Atlas vs FNOC vector speed o 1-day averages $\mathrm{N}=29$ pts 

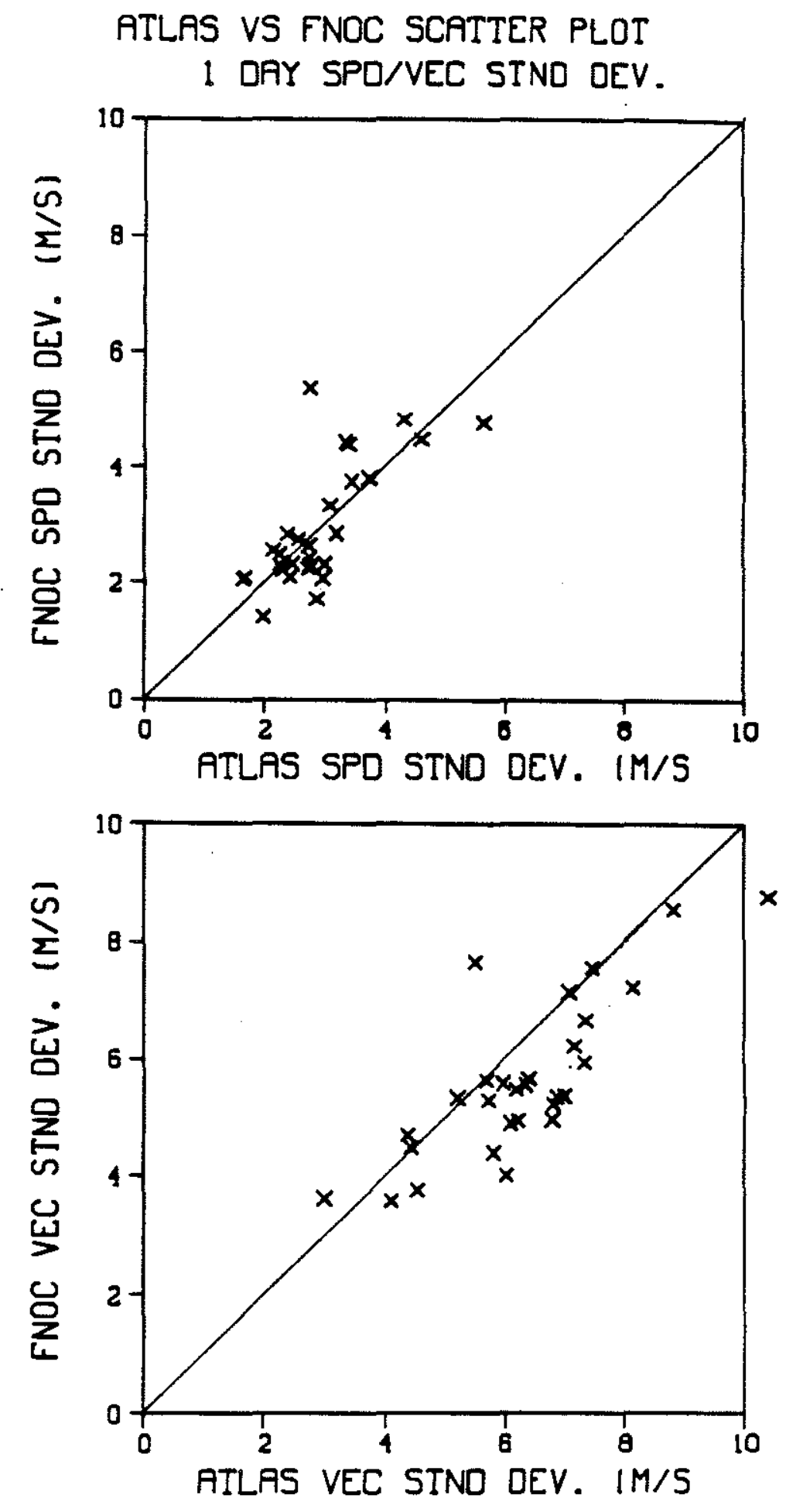

Figure 48: Atlas vs FNOC mean speed and vector standard deviations 1-day averages $\quad \mathrm{N}=29$ pts 


\section{RTLAS VS FNOC SCATIER PLOT}

\section{DAY MEAN SPEED}

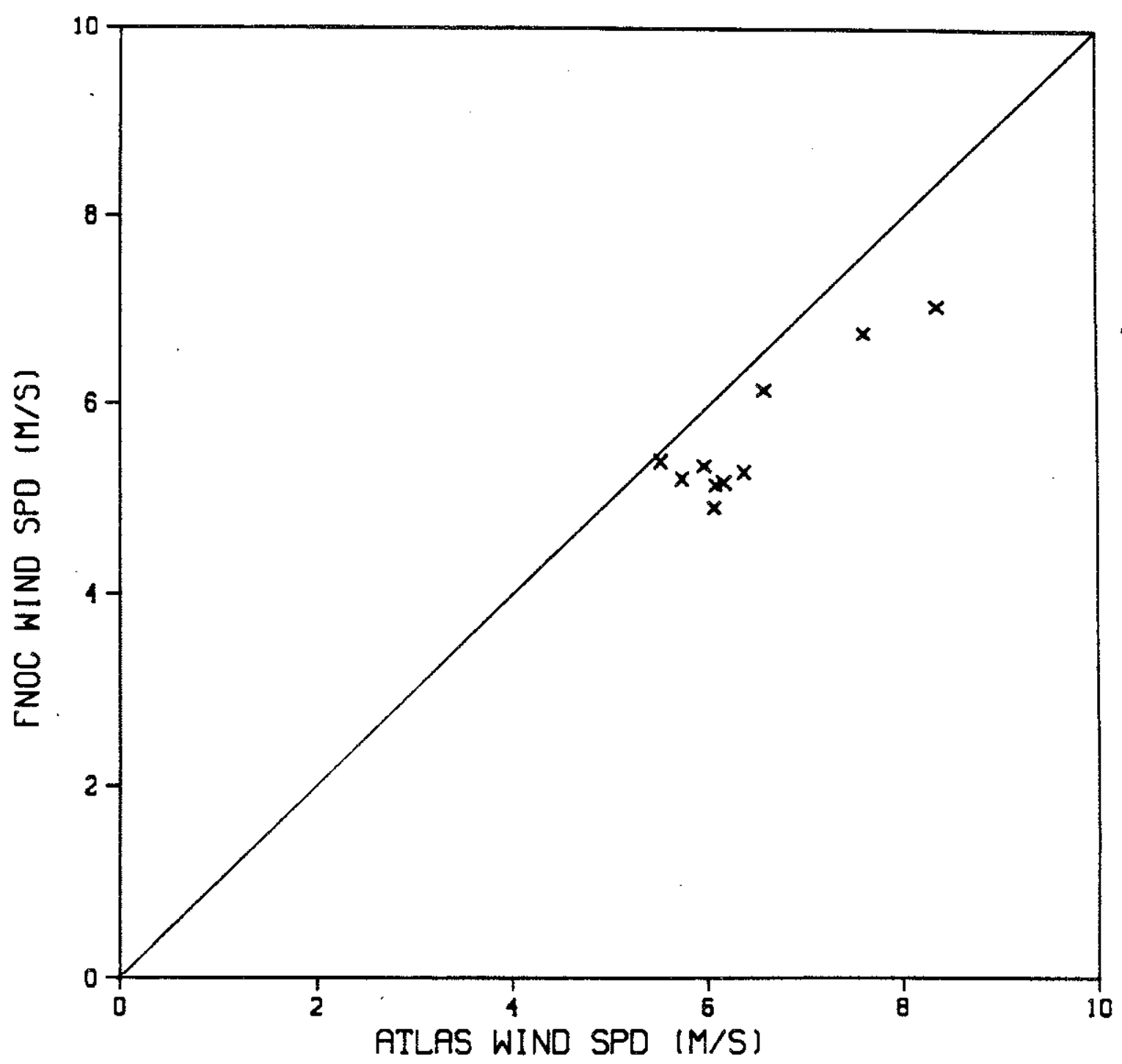

Figure 49: Atlas vs FNOC vector speed - 3-day averages $\mathrm{N}=10 \mathrm{pts}$ 
ATLAS VS FNOC SCATTER PLOT

3 DAY SPD/VEC STND DEV.
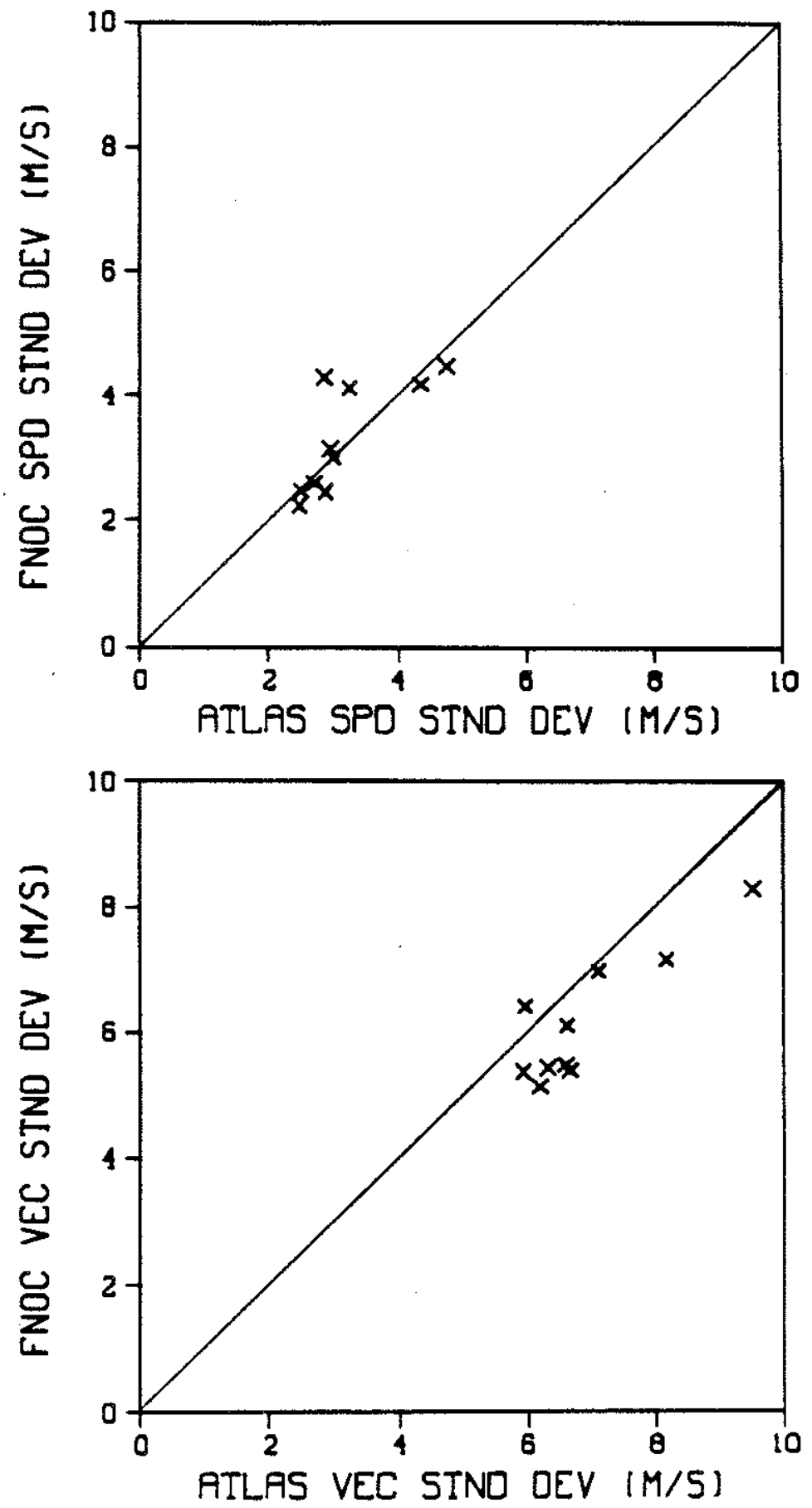

Figure 50: Atlas vs FNOC mean speed and vector standard deviations 3-day averages $\quad \mathrm{N}=10 \mathrm{pts}$ 


\section{ATLAS VS FNOC SCATTER PLOT DAILY RVERAGES}
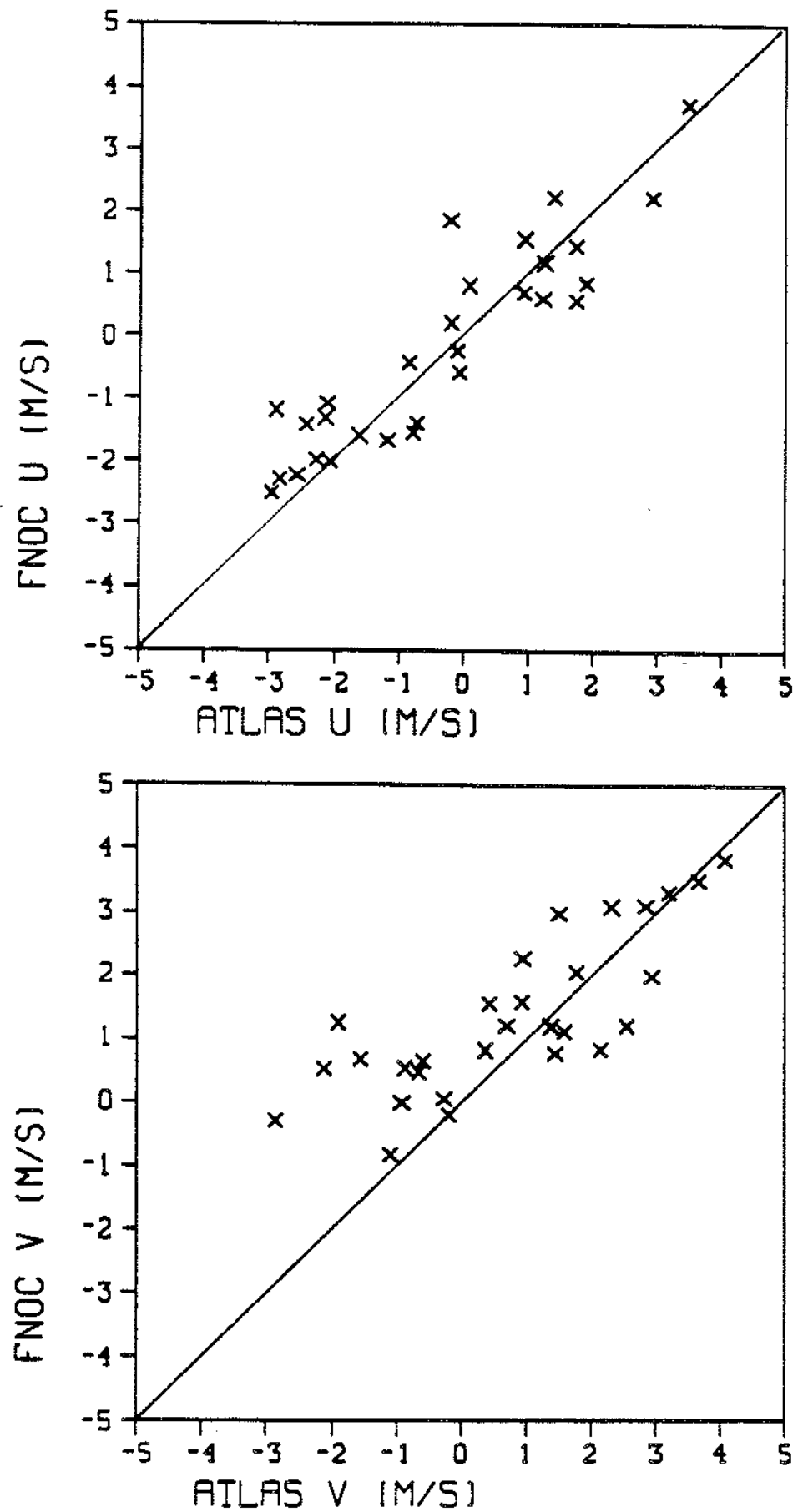

Figure 51: Atlas vs FNOC component velocity - 1-day averages $\mathrm{N}=29$ pts 

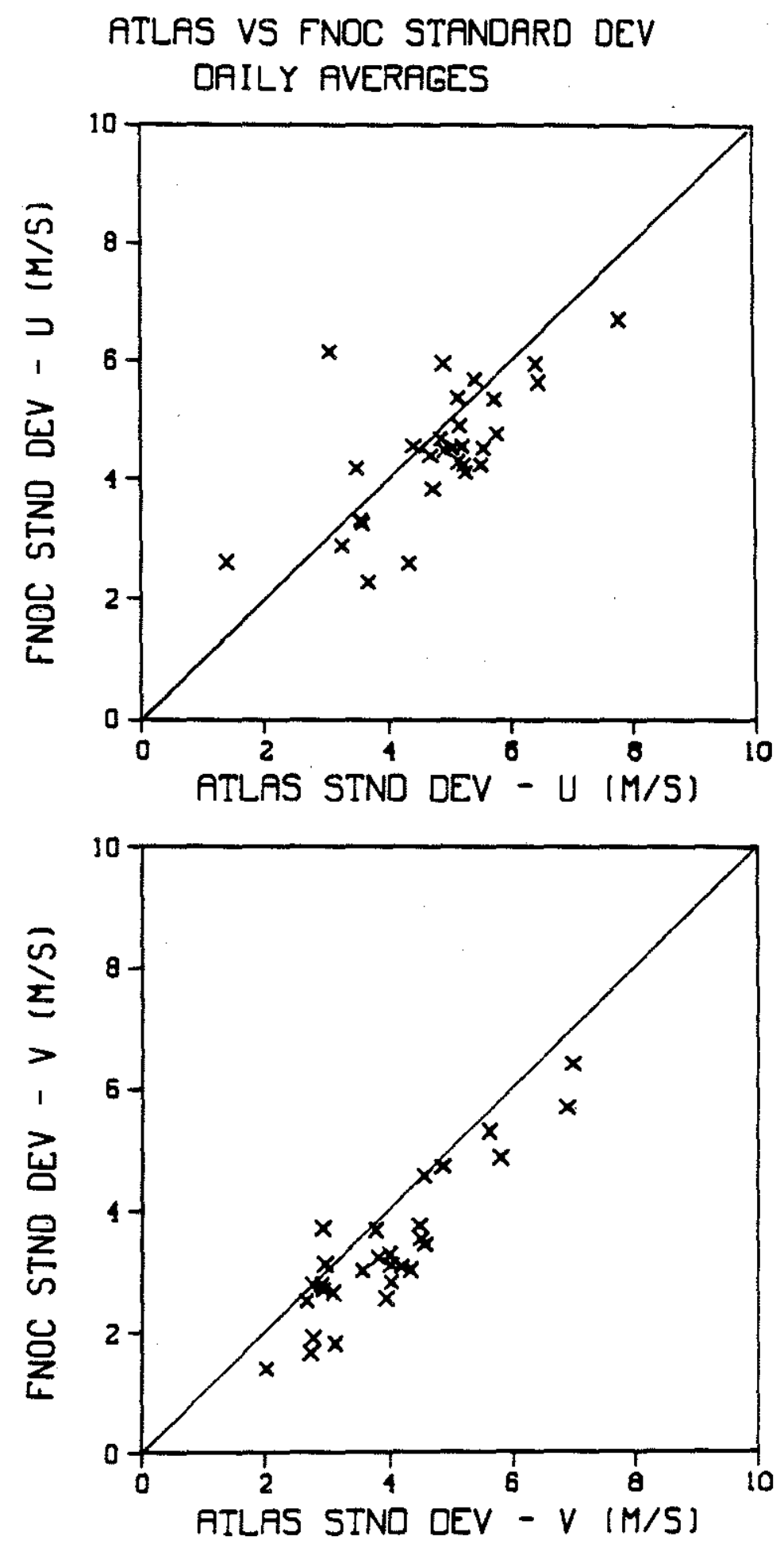

Figure 52: Atlas vs FNOC component standard deviations 1 -day averages $\quad \mathrm{N}=29 \mathrm{pts}$ 

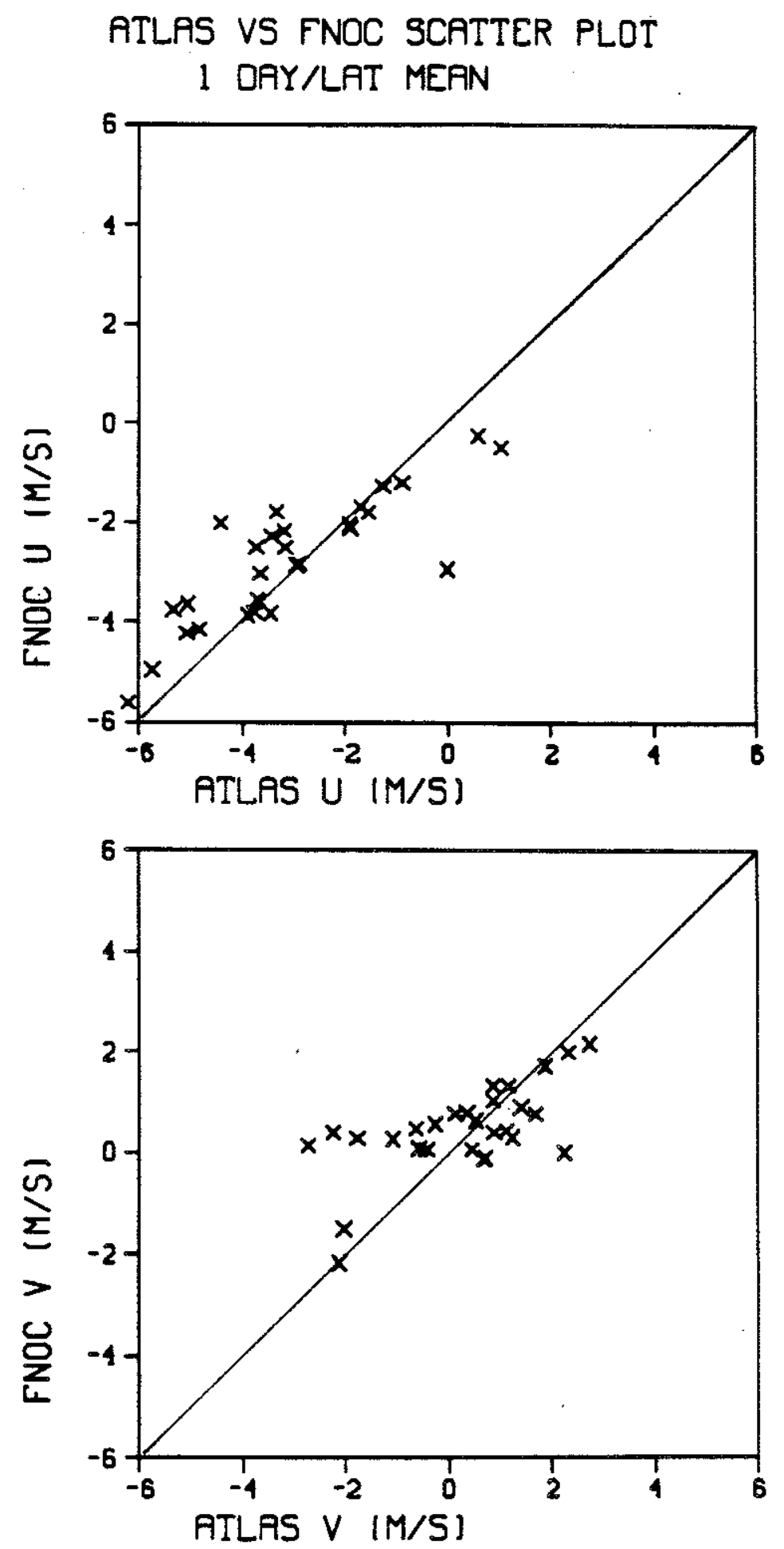

Figure 53: Atlas vs FNOC component velocity - 1-day/10 lat averages $20^{\circ} \mathrm{N}-30^{\circ} \mathrm{N} \quad \mathrm{N}=28 \mathrm{pts}$ 
ATLAS VS FNOC STANDRRD DEV DAY/LAT AVG $20 T 030$
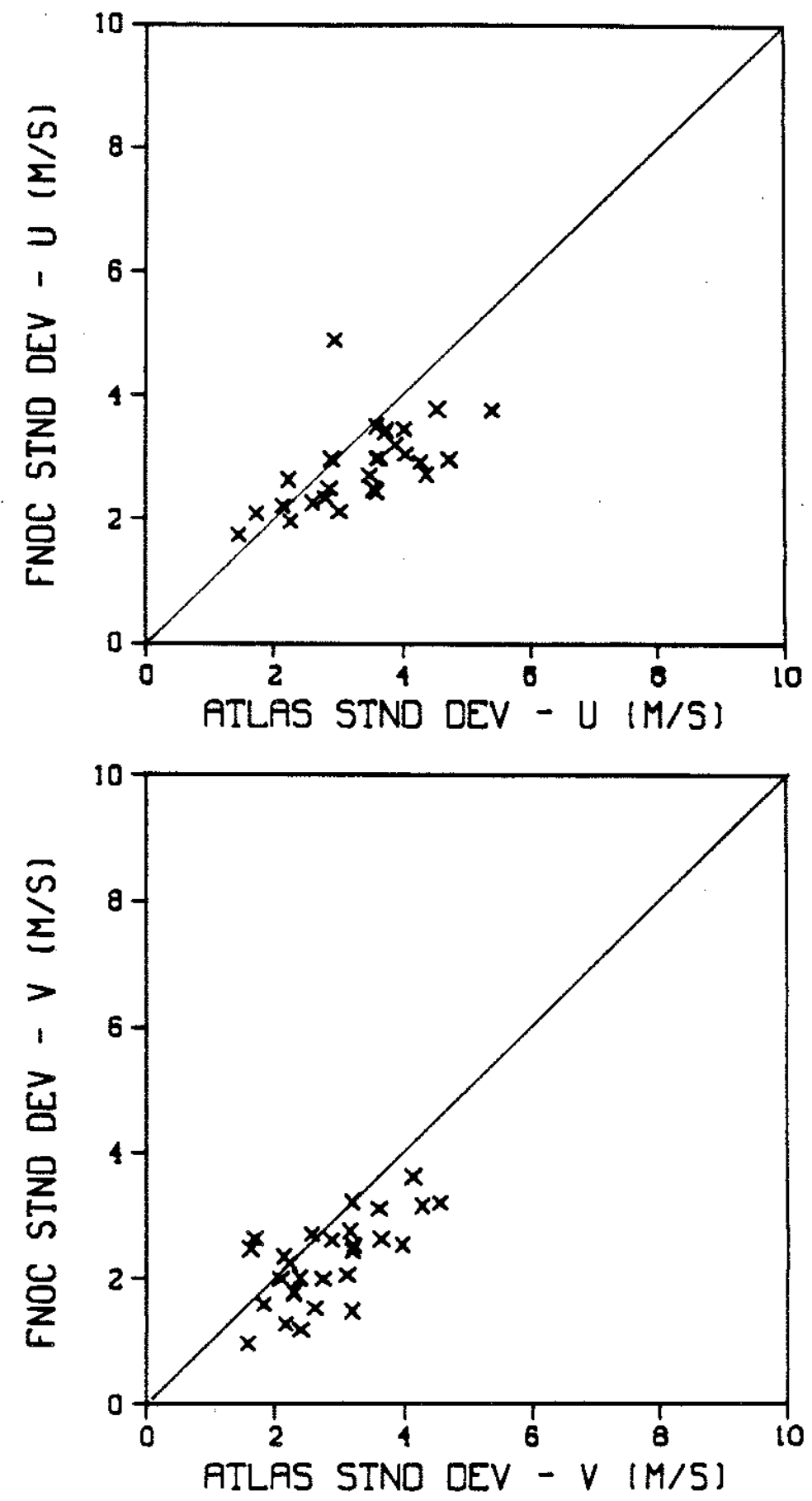

Figure 54: Atlas vs FNOC component standard deviations 1 -day $/ 10^{\circ}$ lat averages $\quad 20^{\circ} \mathrm{N}-30^{\circ} \mathrm{N}$ $\mathrm{N}=28$ pts 

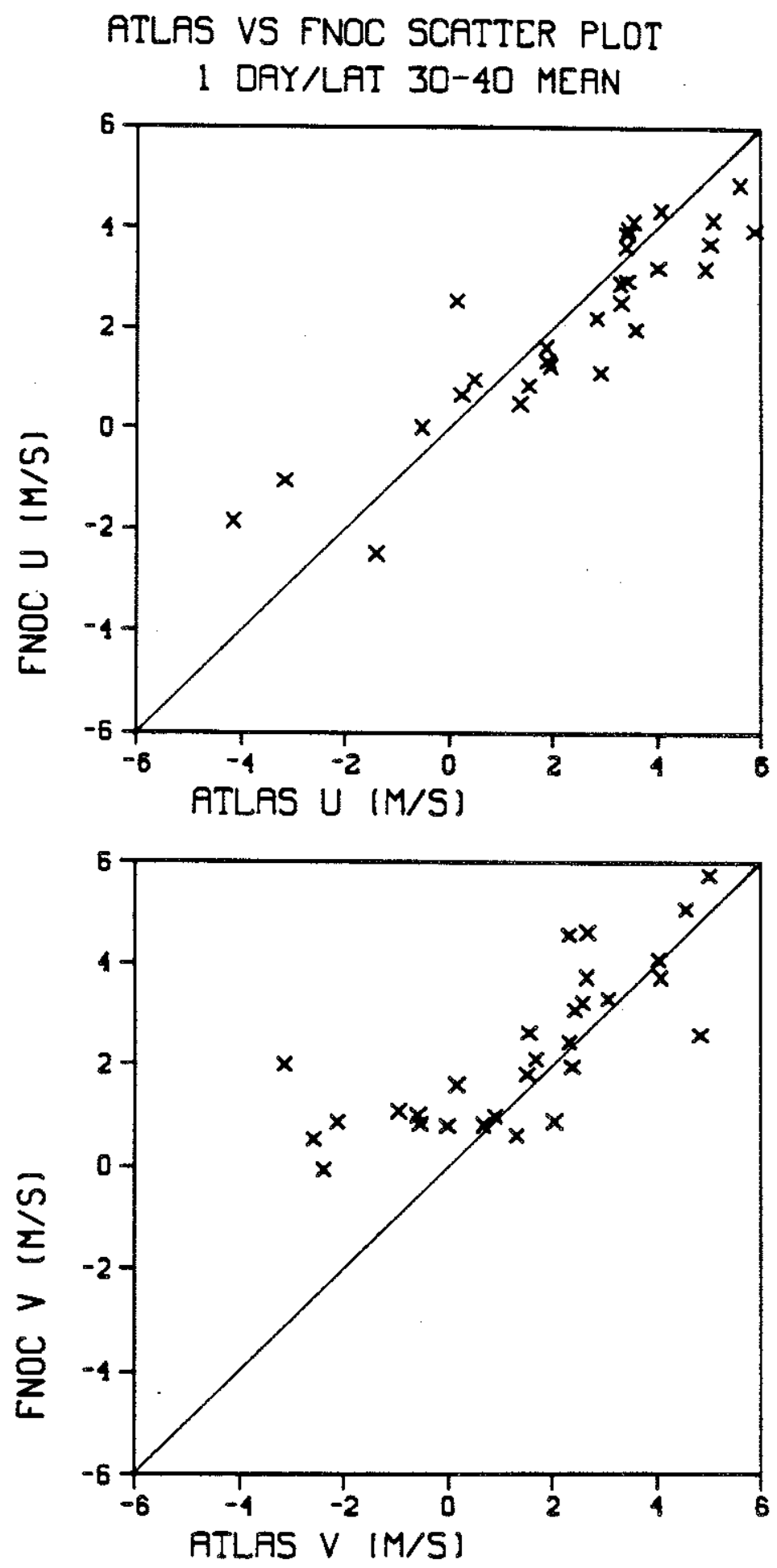

Figure 55: Atlas vs FNOC component velocity - 1-day/10 lat averages $30^{\circ} \mathrm{N}-40^{\circ} \mathrm{N} \quad \mathrm{N}=29$ pts 

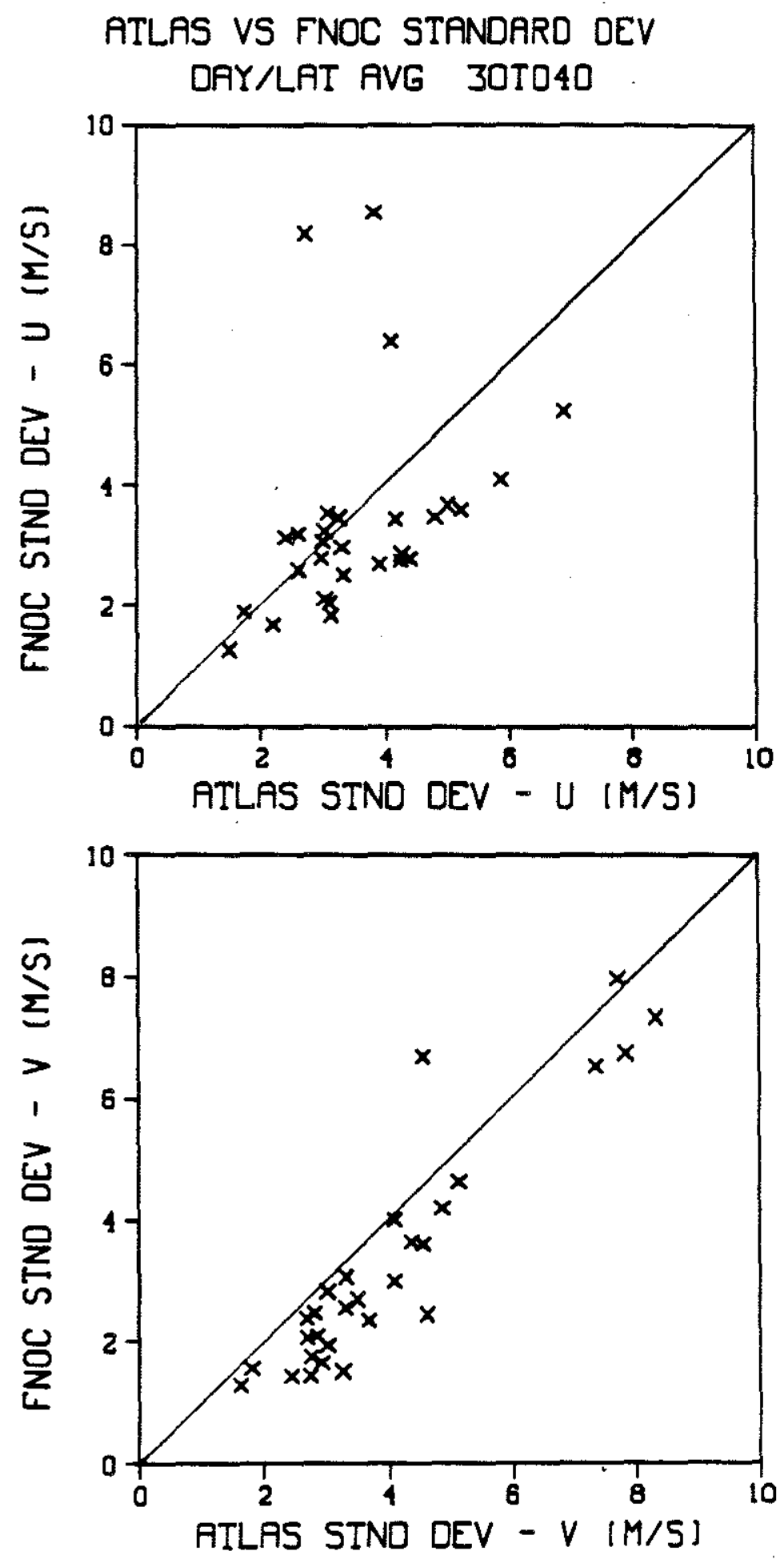

Figure 56: Atlas vS FNOC component standard deviations l-day $/ 10^{\circ}$ lat averages $\quad 30^{\circ} \mathrm{N}-40^{\circ} \mathrm{N}$ $\mathrm{N}=29$ pts 

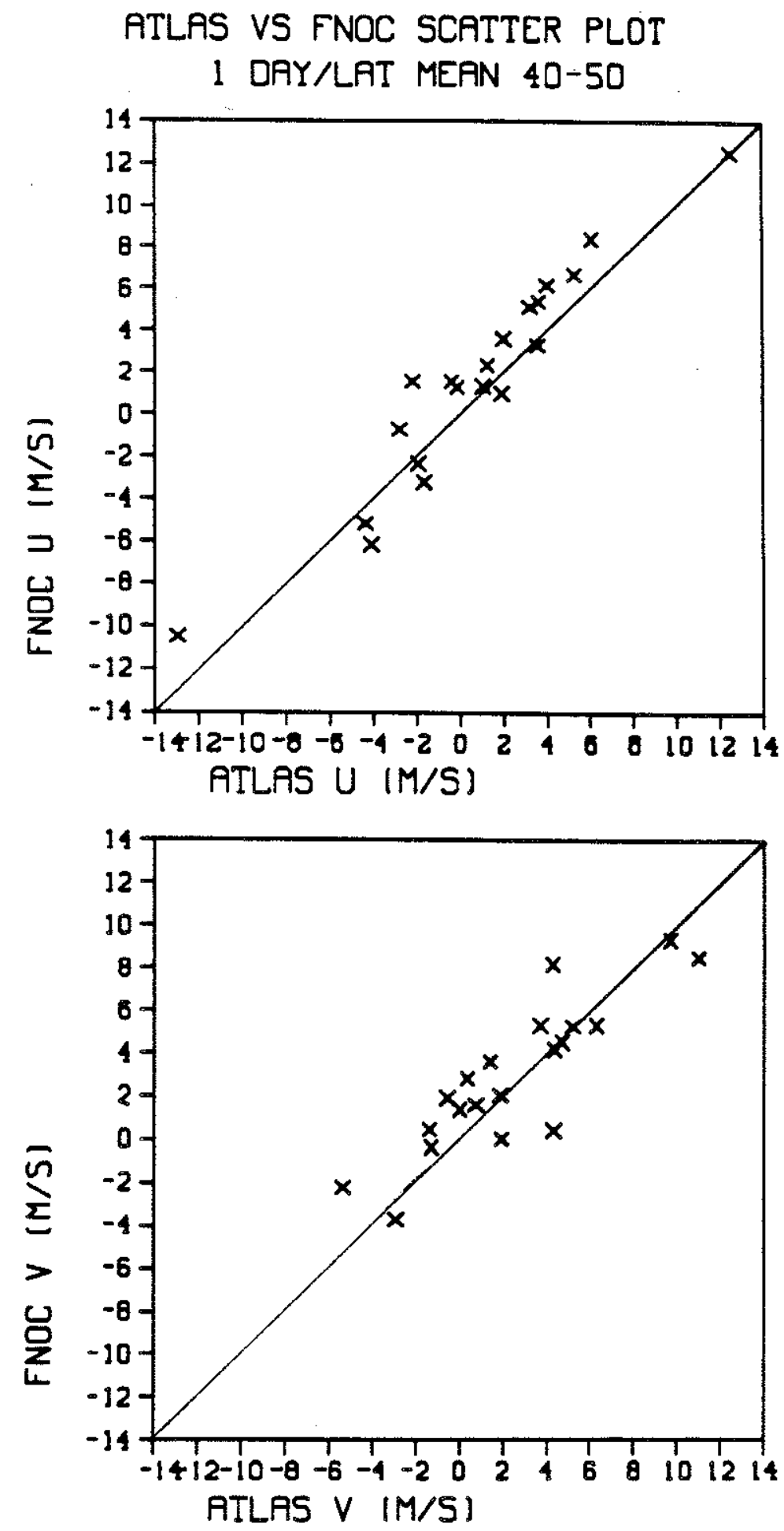

Figure 57: Atlas vs FNOC component velocity - 1-day $/ 10^{\circ}$ lat averages $40^{\circ} \mathrm{N}-50^{\circ} \mathrm{N}$ $\mathrm{N}=22 \mathrm{pts}$ 

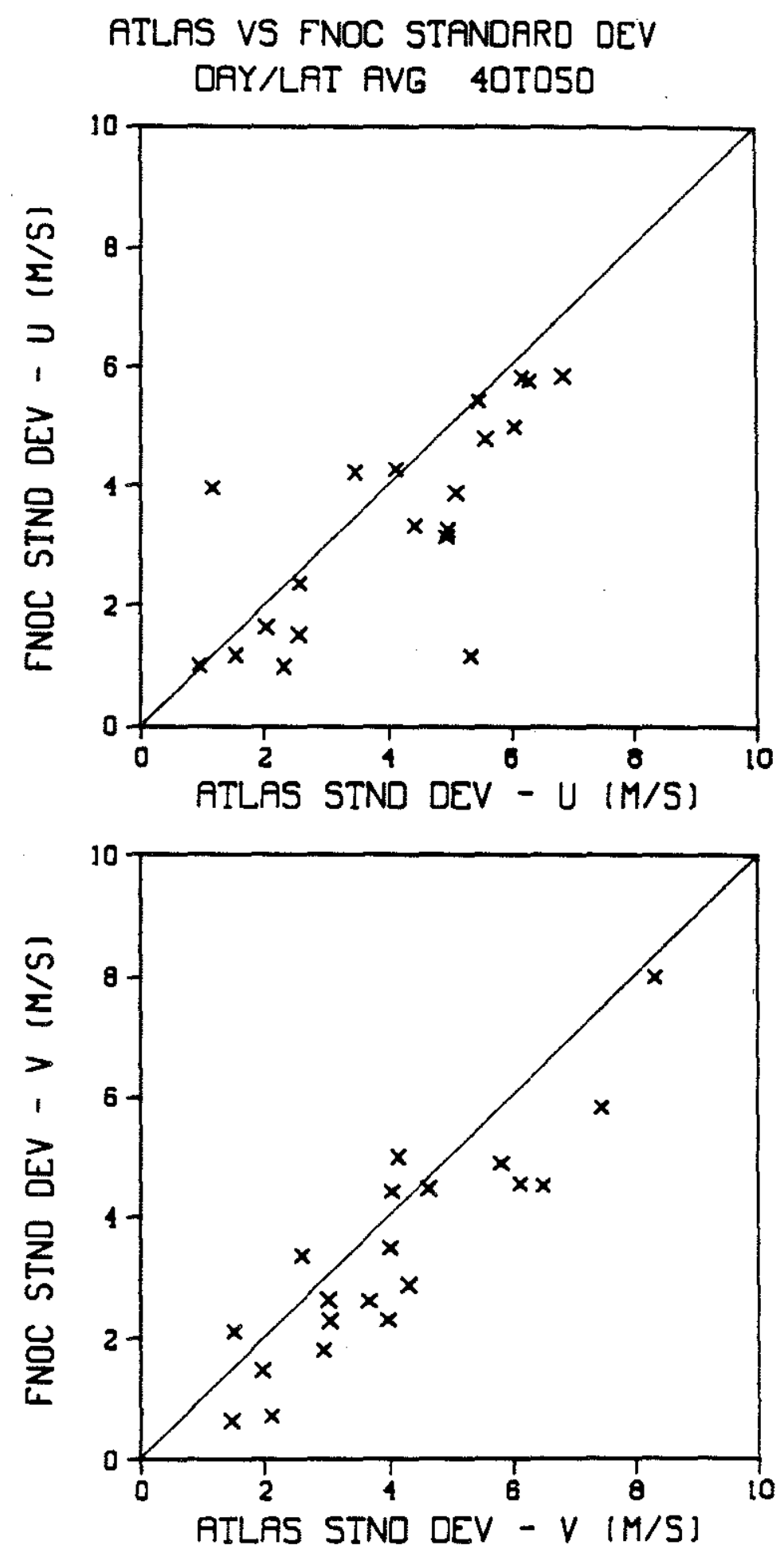

Figure 58: Atlas vs FNOC component standard deviations 1 -day $/ 10^{\circ}$ lat averages $\quad 40^{\circ} \mathrm{N}-50^{\circ} \mathrm{N}$ $\mathrm{N}=22$ pts 
ATLAS-FNOC INDIVIDURL COMPONENTS
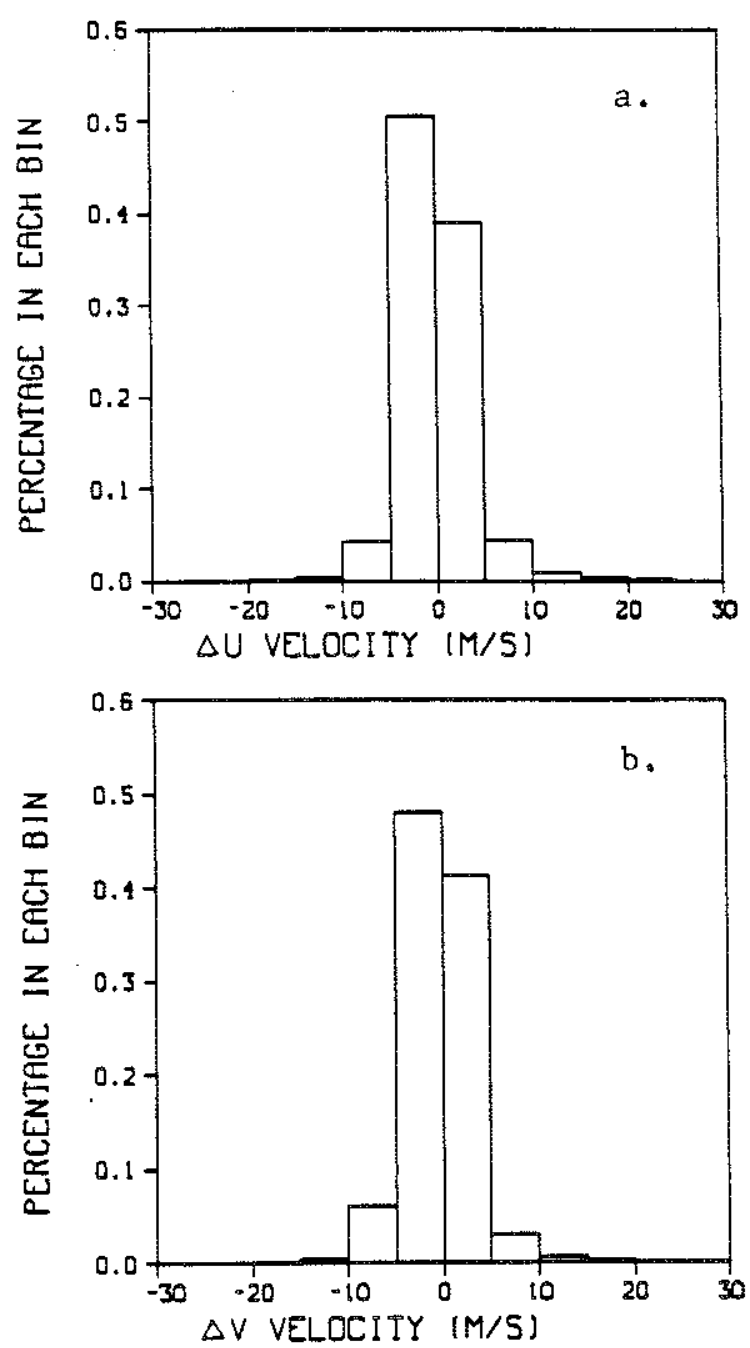

ATLAS-FNOC INDIVIOUAL VECTORS
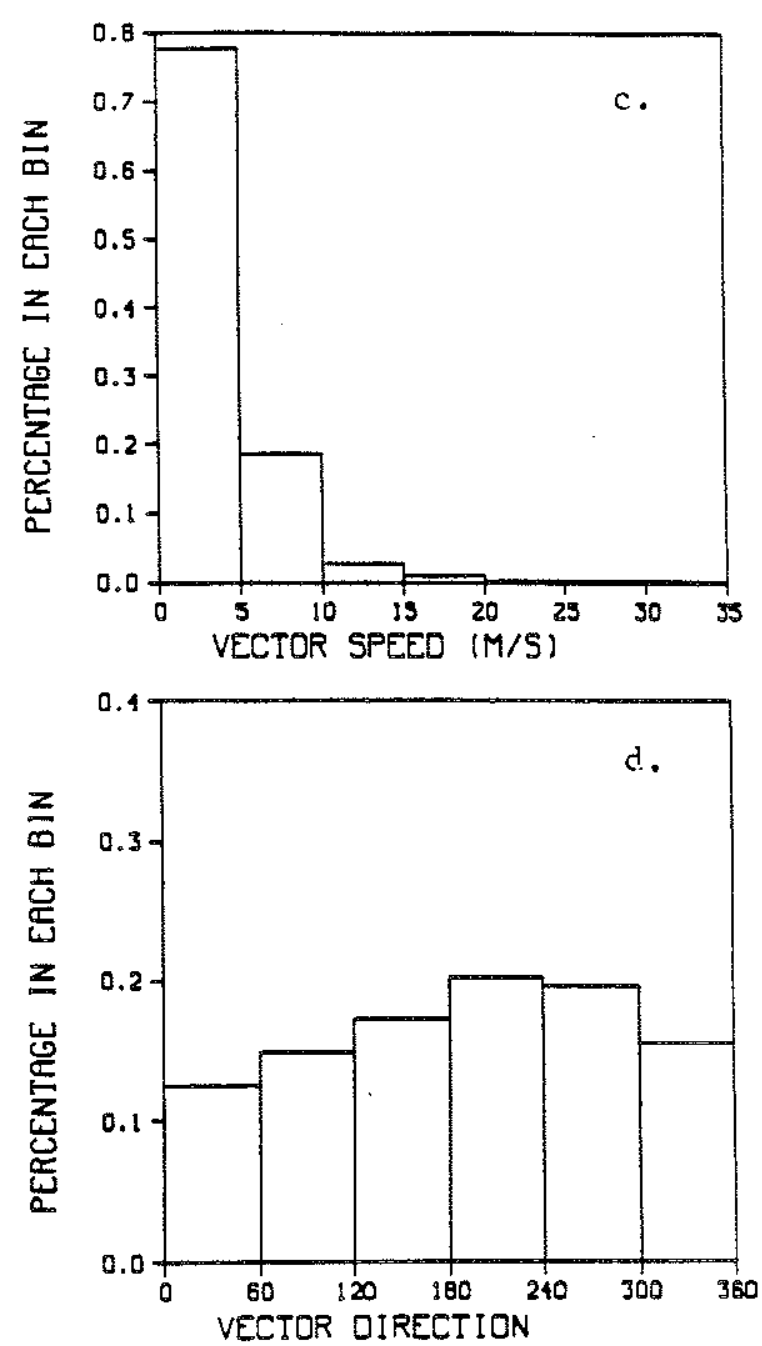

Figure 59: Distribution of all individual Atlas $-F N O C$ difference field vectors. a. $\Delta U$ velocity, b. $\Delta V$ velocity, c. vector speed, d. vector direction $\mathrm{N}=8214$ pts 
6642

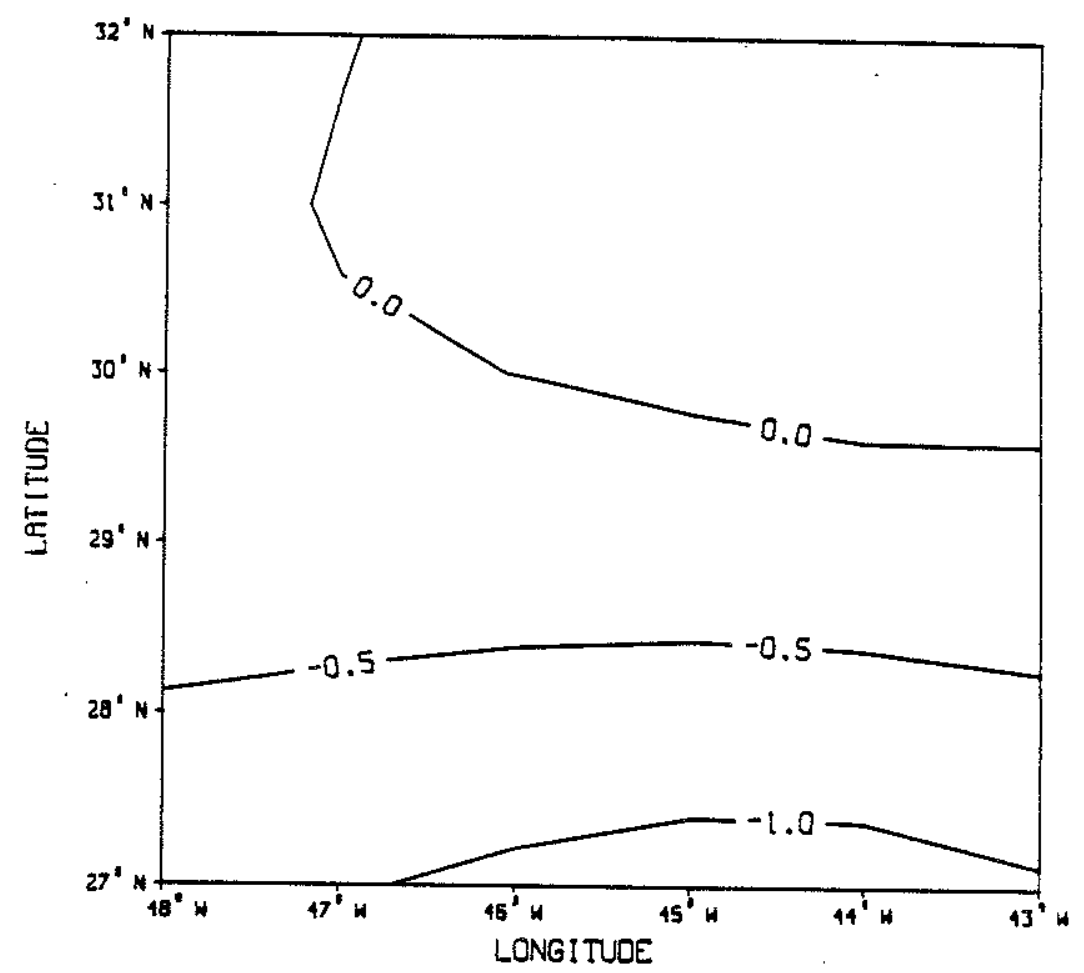

6643

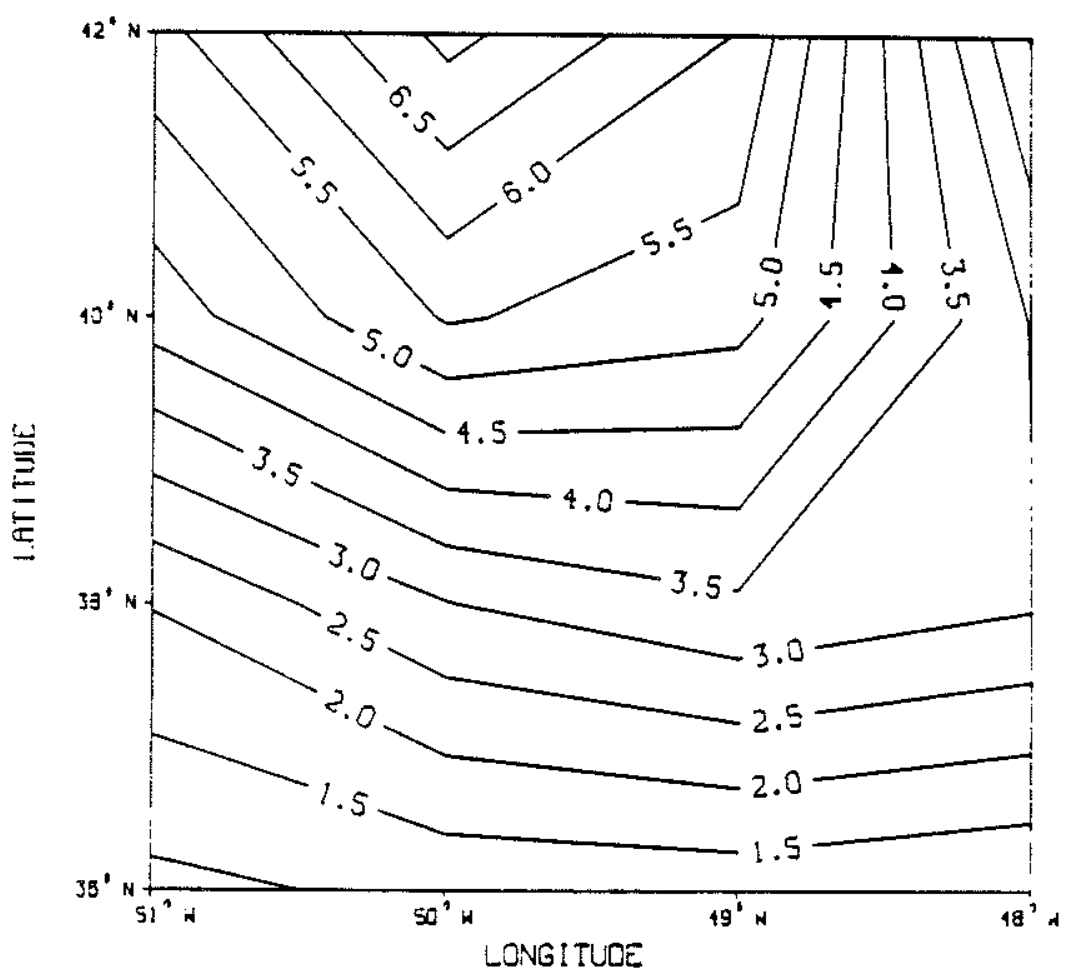

Figure 60: Atlas wind stress curl - Rev 664 boxes 2 and 3 $\left(\mathrm{x} 10^{-7} \mathrm{~N} / \mathrm{m}^{3}\right)$ 
WIND STRESS CURL

7151 ATLAS $\times 10-7$

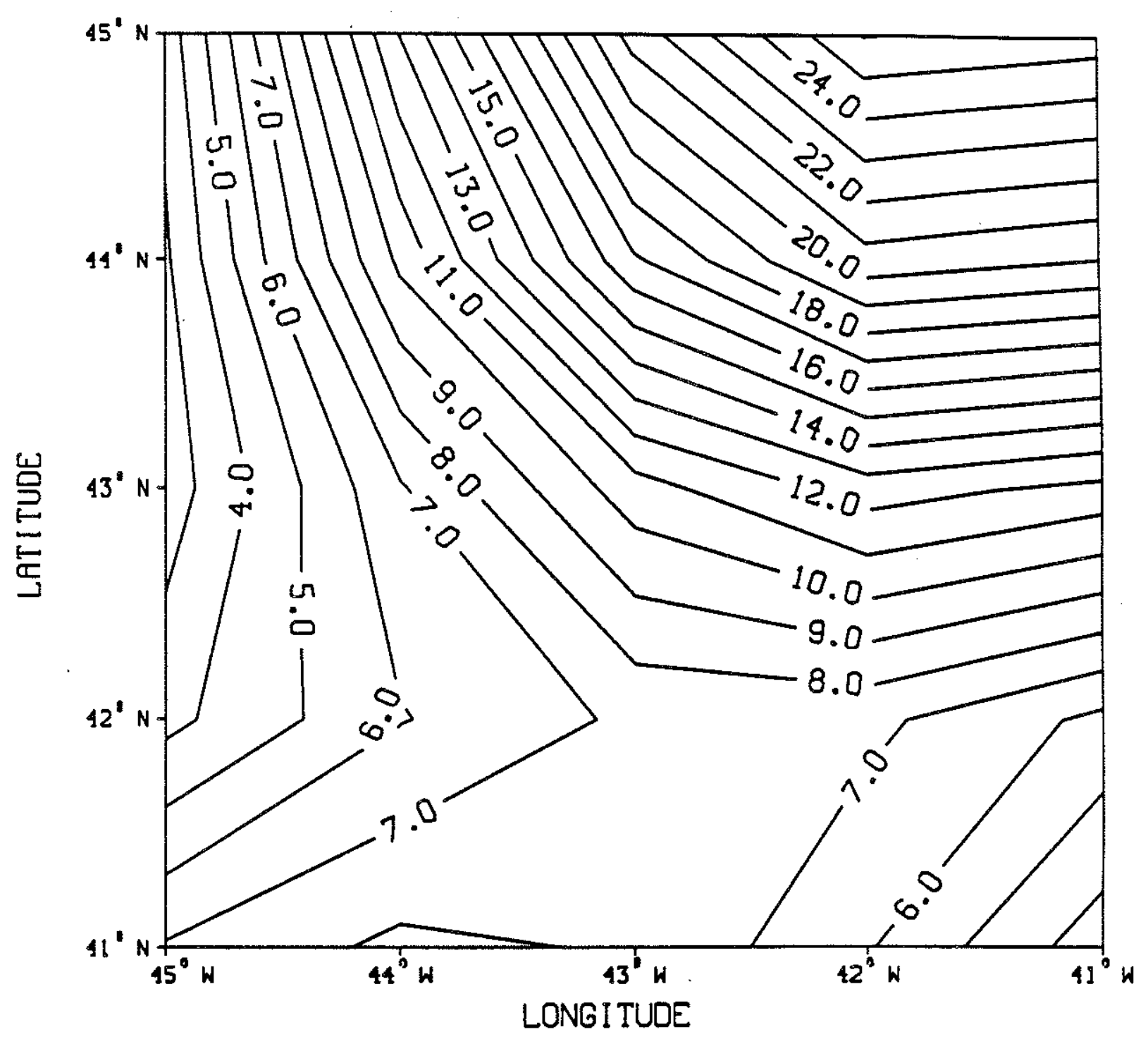

Figure 61: Atlas wind stress cur1 - Rev 7151 $\left(\times 10^{-7} \mathrm{~N} / \mathrm{m}^{2}\right)$ 
10592 ATLAS $\times 10-7$

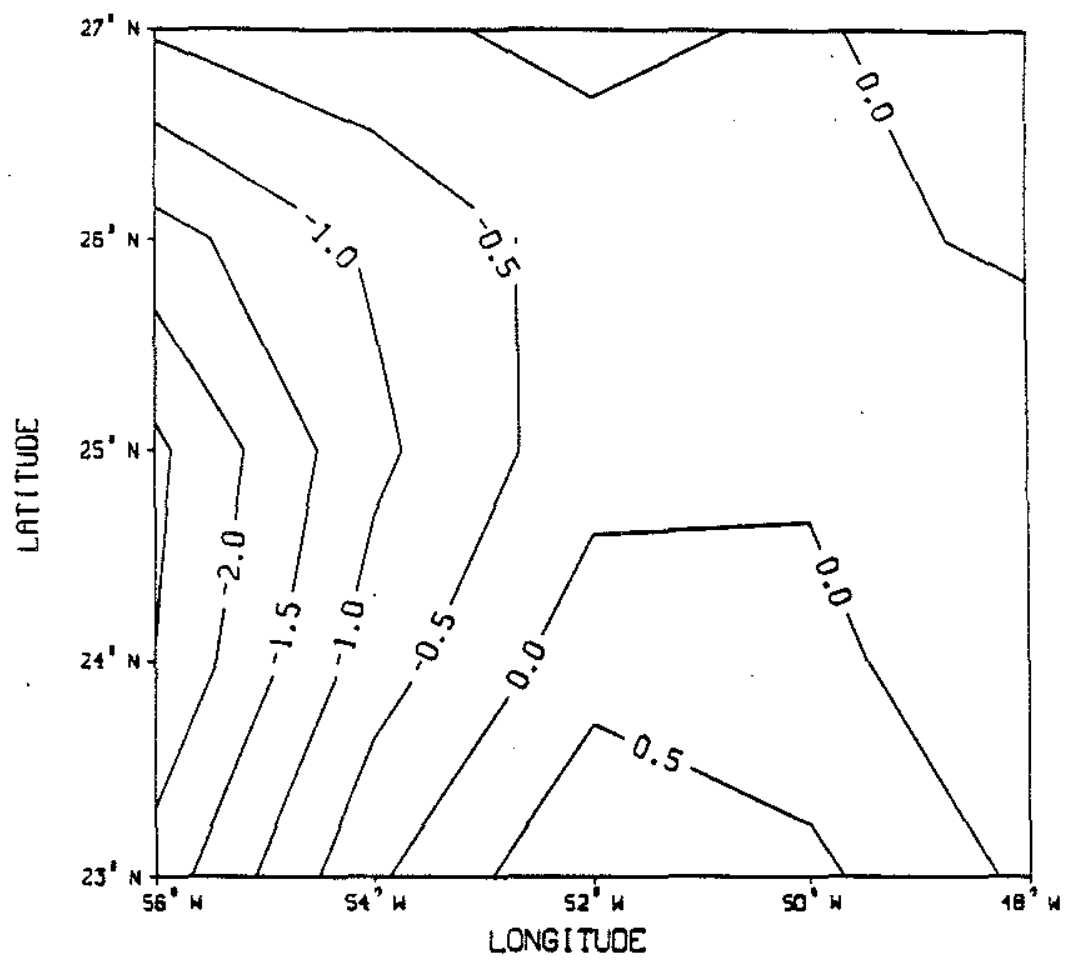

10592 FNOC $\times 10-7$

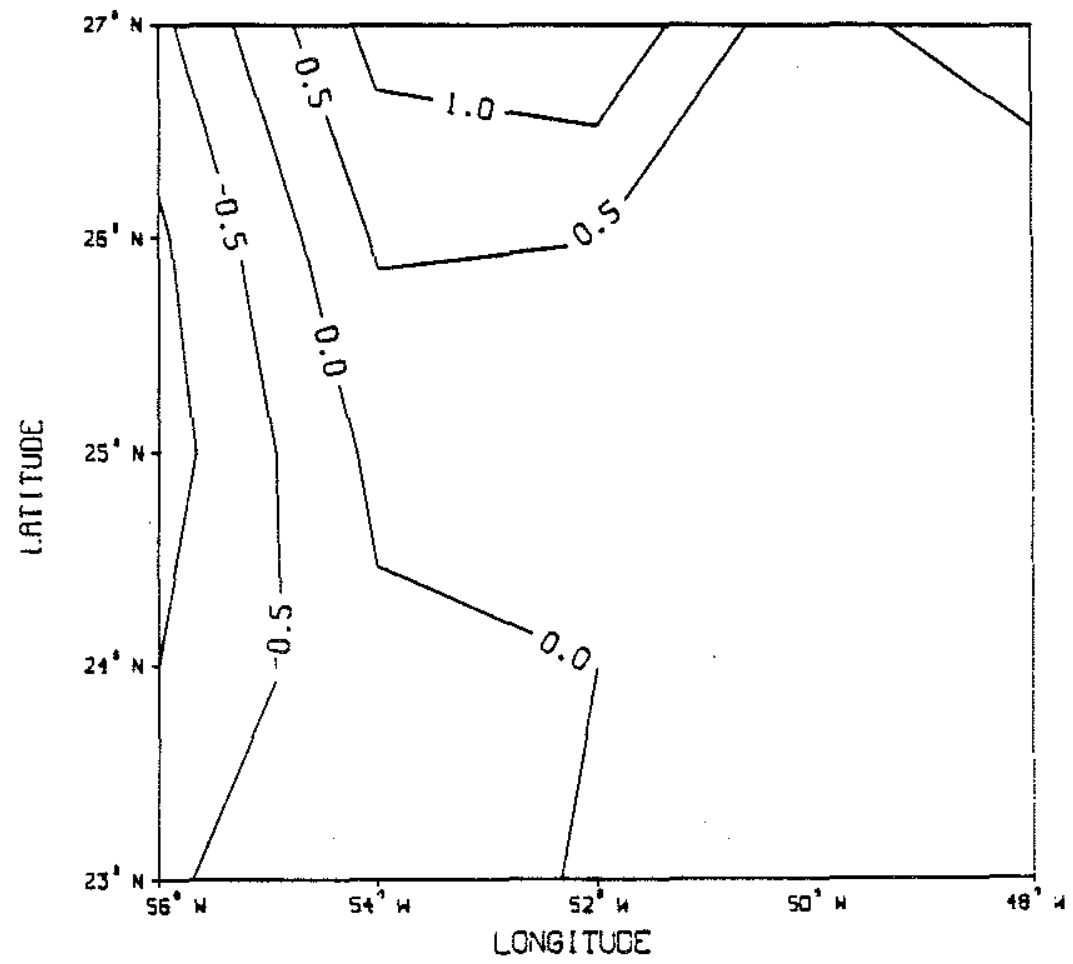

Figure 62: Atlas and FNOC wind stress curl - Rev 10592 $\left(\times 10^{-7} \mathrm{~N} / \mathrm{m}^{2}\right)$ 

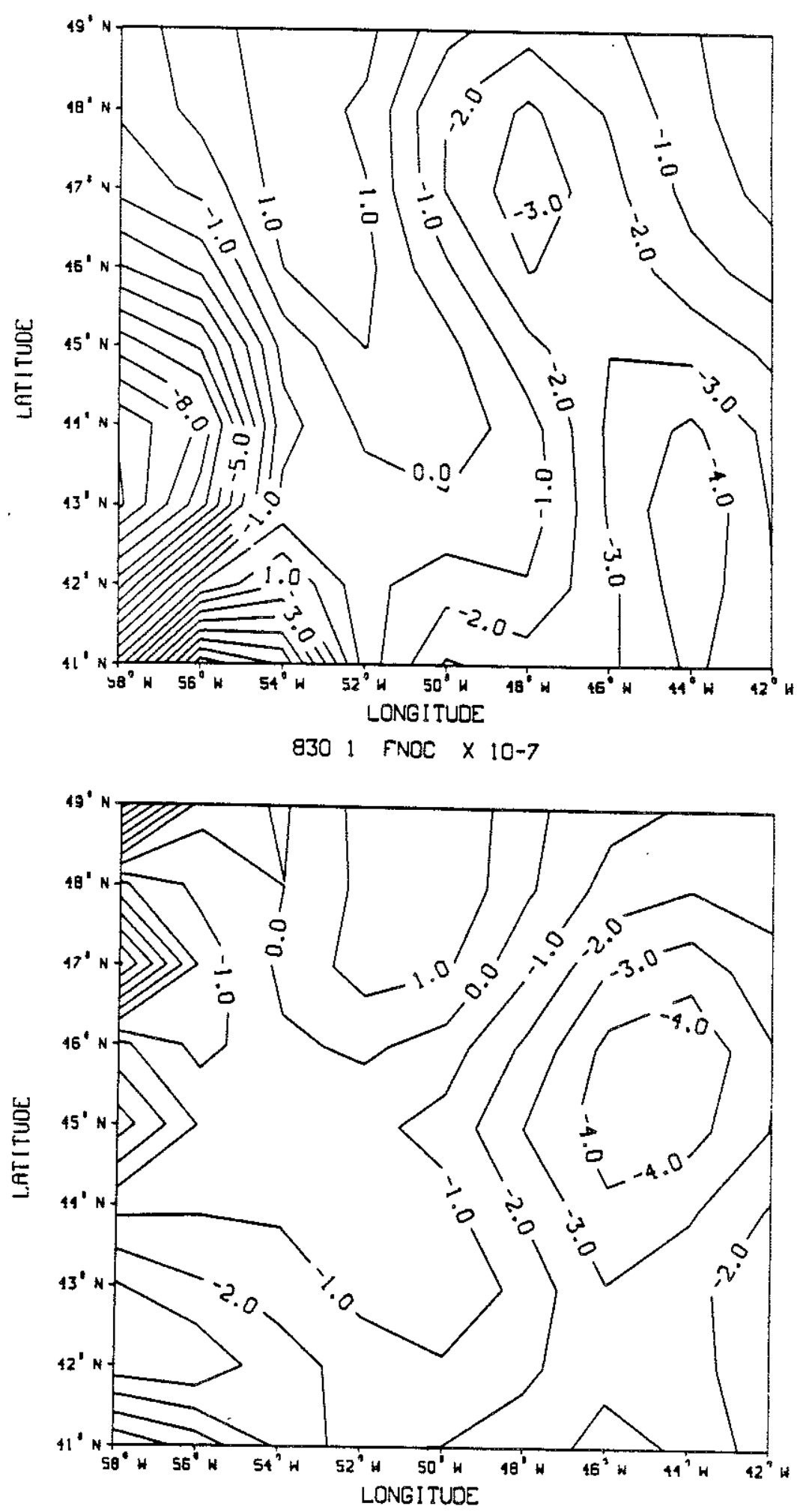

Figure 63: Atlas and FNOC wind stress curl - Rev 8301 $\left(\mathrm{x} 10^{-7} \mathrm{~N} / \mathrm{m}^{3}\right)$ 


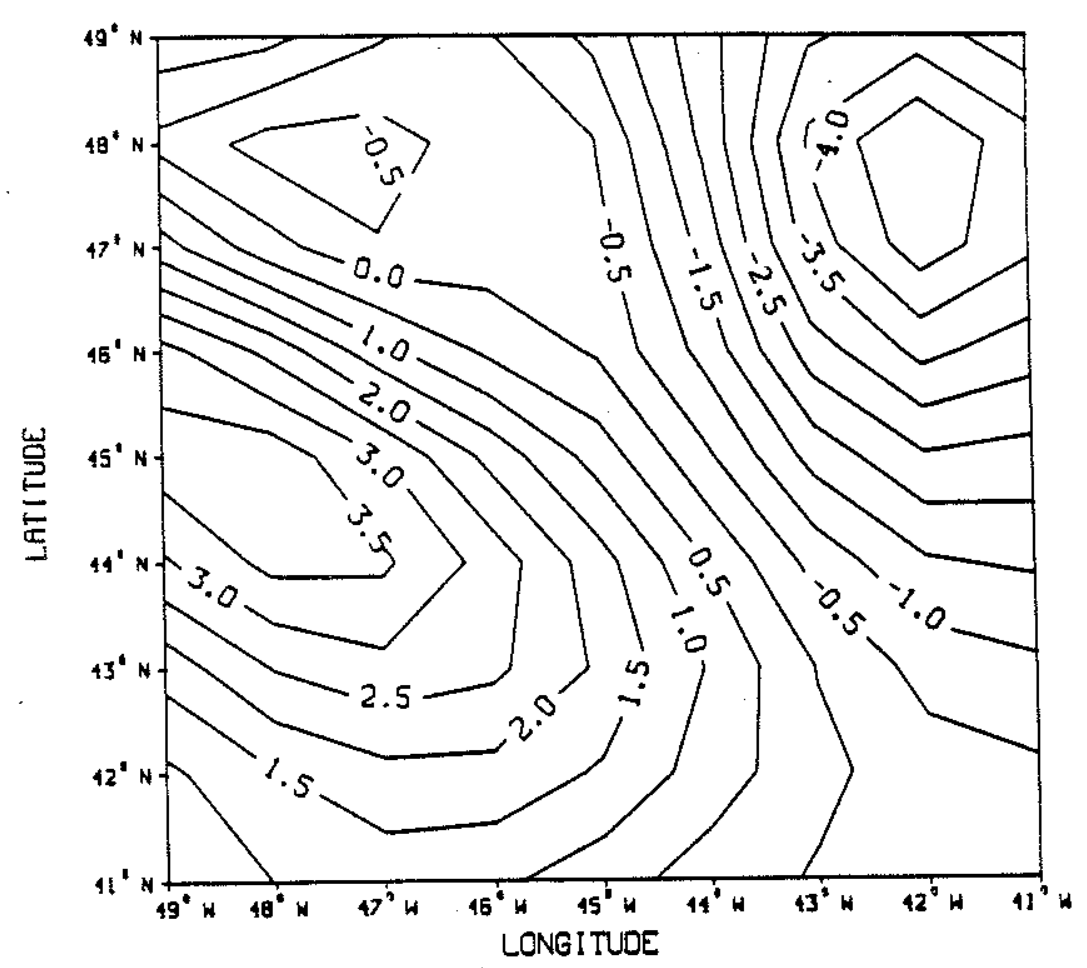

10082 FNOC $\times 10-6$

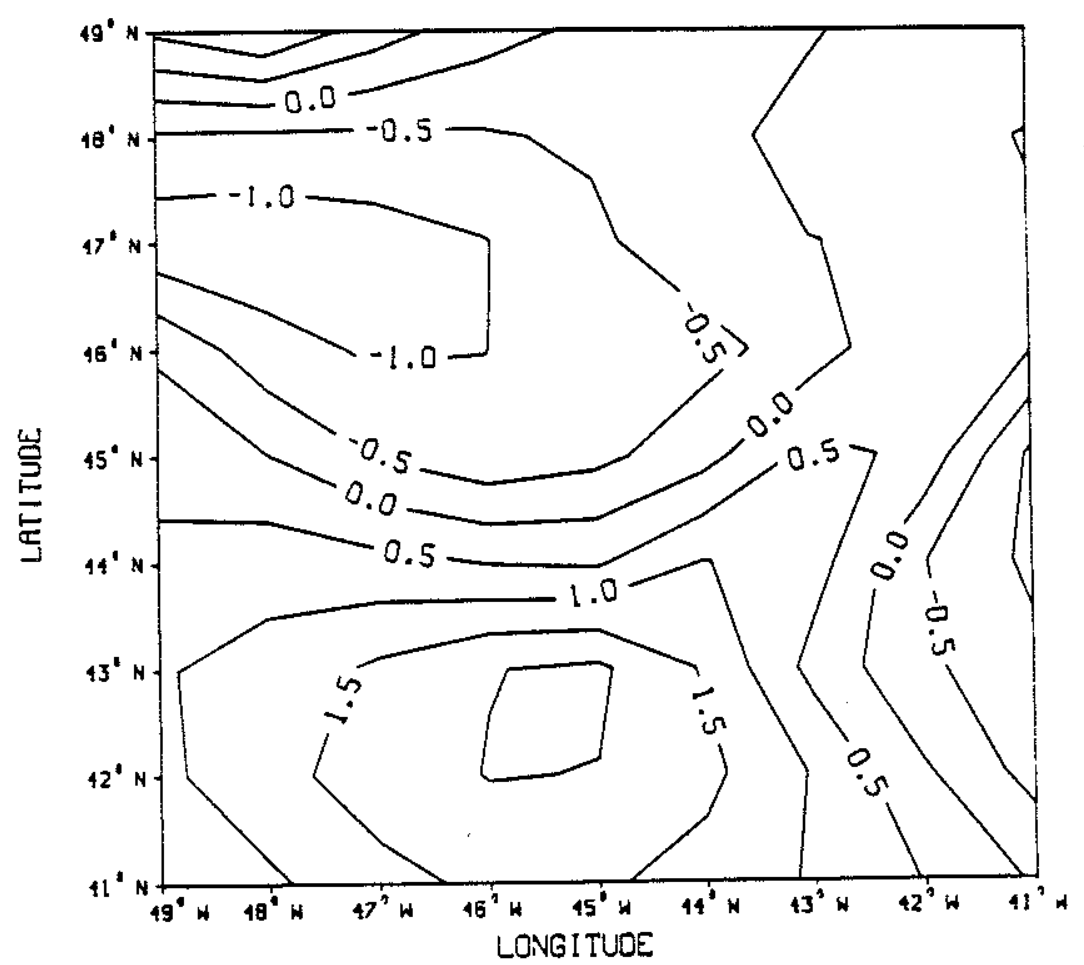

Figure 64: Atlas and FNOC wind stress cur1 - Rev 10082 $\left(\mathrm{x} 10^{-6} \mathrm{~N} / \mathrm{m}^{3}\right)$ 

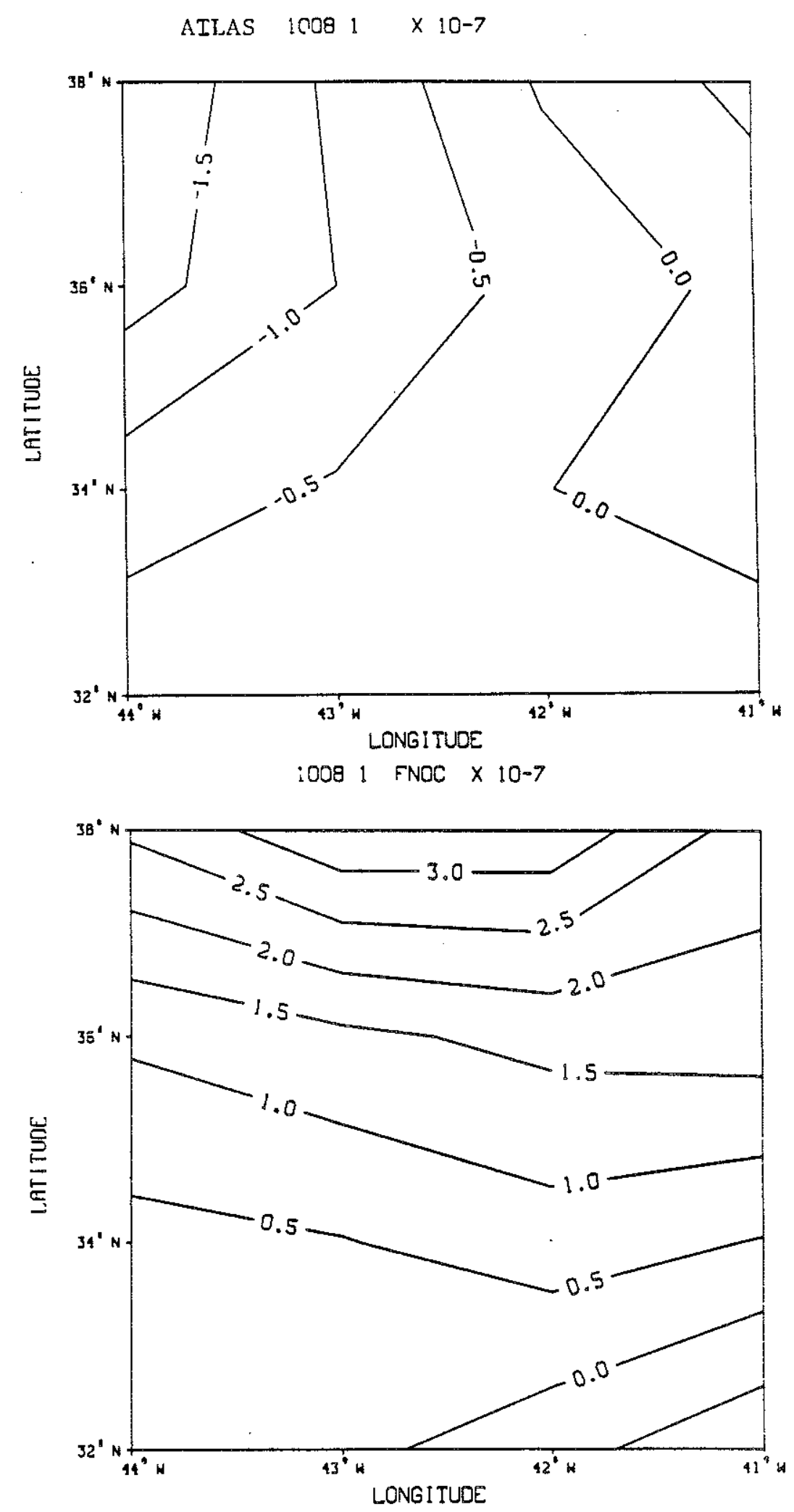

Figure 65: Atlas and FNOC wind stress curl - Rev 10081 $\left(\times 10^{-7} \mathrm{~N} / \mathrm{m}^{3}\right)$ 


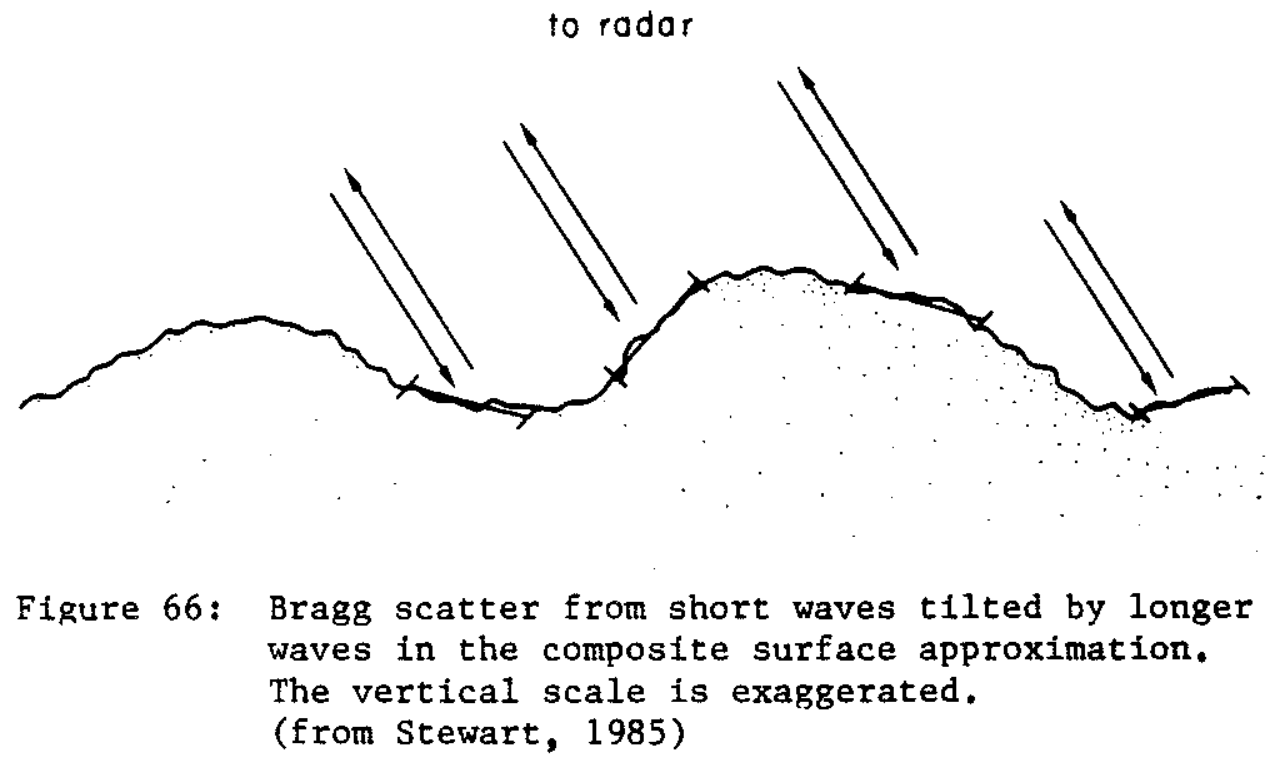




\section{ACKNOWLEDGEMENTS}

I would like to thank the following persons and organizations for their contributions to my thesis:

My advisor, Dr. Carl Wunsch - for guidance, knowledge, helpful criticism, and unswerving support.

Charmaine King and Barbara Grant - for invaluable computing assistance and programming advice, putting up with numerous interruptions, and coming through on short notice. Charmaine and Barbara are supported in part by ONR grant \#N0001485-J-1241.

The students, post-docs, and staff of the 13th floor of the Green Building (especially my office mates) - for scientific discussions, lunch and tea-hour breaks, an international perspective, lots of laughs, and loads of encouragement.

LCDR Paul E. Sullivan - for running interference on all that Navy paperwork and for lots of encouragement.

The United States Navy - for funding my stay at M.I.T. and the Woods Hole Oceanographic Institution through the Naval Postgraduate School Civilian Institutions program.

My parents, Robert M. and Barbara Holderied - for love, understanding, and support over all these years. 